\title{
Transmission dynamics of infectious diseases on transportation networks
}

\section{Ph.D. Thesis}

\author{
Diána H. Knipl \\ Supervisor: \\ Gergely Röst, Ph.D.
}

\author{
Doctoral School in Mathematics \\ and Computer Science
}

University of Szeged, Bolyai Institute

Szeged

2014 



\section{Acknowledgment}

I would like to thank my professors, Prof. László Hatvani, Prof. Tibor Krisztin and Dr. Gergely Röst for introducing to me the theory of differential equations. I am heartily thankful to my supervisor, Gergely Röst for encouraging me to start an academic career. His constant support and the challenging problems he posed motivated me a lot throughout my Ph.D. studies.

I would like to express my special gratitude to my high school math teacher, Dr. Sándor Katz. His endless devotion to teaching and his students evoked my affection for studying higher mathematics.

I am also grateful for the support of the Doctoral School in Mathematics and Computer Science and for the assistance I got from Prof. Vilmos Totik and the Analysis and Stochastics Research Group of the Hungarian Academy of Sciences. I extend my sincere thanks to all former and present members of the EPIDELAY research group for making the department a fun place to work in. 



\section{Contents}

List of Figures $\quad$ iii

1 Introduction 1

$\begin{array}{lll}2 & \text { Differential equations with dynamically defined delay term } & \mathbf{7}\end{array}$

2.1 General formulation of the system . . . . . . . . . . . . . . . . 7

2.2 Fundamental properties . . . . . . . . . . . . . . . . . . . 9 9

2.3 The autonomous case . . . . . . . . . . . . . . . . . . . 13

2.3 .1 Basic properties . . . . . . . . . . . . . . . . . . 13

2.3.2 Equilibria and linearization . . . . . . . . . . . . . . . . 14

2.4 A model from population dynamics with temporary separation . . . . . . 16

3 A delay model for the spread of pandemics between connected regions $\quad 18$

3.1 Introduction . . . . . . . . . . . . . . . . . . . . . . . . 18

3.2 Model description . . . . . . . . . . . . . . . . . . . . . . . . . 20

3.3 Basic properties of the model . . . . . . . . . . . . . . . . . . 27

3.4 The basic reproduction number . . . . . . . . . . . . . . . . . . 33

3.4.1 The next generation matrix . . . . . . . . . . . . . . 40

3.4.2 The dependence of $\mathcal{R}_{0}$ on key model parameters . . . . . . . . . 49

3.4.3 $\mathcal{R}_{0}$ as the threshold quantity for epidemic outbreaks . . . . . . . 52

3.5 Parametrization for influenza . . . . . . . . . . . . . . . . 54

3.6 Prototype origin-destination pairs . . . . . . . . . . . . 56

3.6.1 Canada - China: The case of asymmetric populations . . . . . . . 58

3.6.2 Canada - United Kingdom: The symmetric case . . . . . . . . . . . 59

3.6.3 Canada - Mexico: The case of asymmetric travel . . . . . . . . . . 60

3.6.4 Fitting the model to the $2009 \mathrm{~A}(\mathrm{H} 1 \mathrm{~N} 1)$ pandemic . . . . . . . . . . 62

3.7 Conclusion . . . . . . . . . . . . . . . . . . . . . . 63

4 Epidemic models with travel-related infection $\quad 65$

4.1 An epidemic model with entry screening . . . . . . . . . . . . . . . . 65

4.1.1 The compact form of the model . . . . . . . . . . . . . . . . . 69 
4.2 Multiregional SIR model with general infection term . . . . . . . . . . . 73

4.2.1 The compact form of the model . . . . . . . . . . . . . . . 76

4.2 .2 Basic properties of the model . . . . . . . . . . . . . 78

5 Backward bifurcation in SIVS model with immigration of non-infectives $\quad \mathbf{8 4}$

5.1 Introduction . . . . . . . . . . . . . . . . . . . . 84

5.2 Model description . . . . . . . . . . . . . . . . . . . . . . . . 87

5.3 Basic properties of the model . . . . . . . . . . . . . . . 88

5.4 Endemic equilibria . . . . . . . . . . . . . . . . . . . . . 90

5.5 Stability and global behavior . . . . . . . . . . . . . . . 94

5.6 The influence of immigration on the backward bifurcation . . . . . . . . 97

5.7 Revisiting the three-dimensional system . . . . . . . . . . . . . 105

5.8 Conclusion . . . . . . . . . . . . . . . . . . . . . 106

$\begin{array}{ll}\text { Summary } & 109\end{array}$

$\begin{array}{lr}\text { Összefoglalás } & 115\end{array}$

$\begin{array}{ll}\text { Bibliography } & 121\end{array}$ 


\section{List of Figures}

3.1 Flow chart of disease transmission and travel dynamics of the SEAIR model. 23

3.2 The dependence of $\mathcal{R}_{0}$ on $\beta^{T} \ldots \ldots \ldots \ldots \ldots \ldots$

3.3 The dependence of $\mathcal{R}_{0}$ on $\frac{1}{\gamma_{1}}$ and $\frac{1}{\gamma_{2}}$ in the case of $\gamma_{1}=\gamma_{2}$ and $\tau>0 \ldots . .51$

3.4 The dependence of some elements of the next generation matrix on $\frac{1}{\gamma_{1}}$ and $\frac{1}{\gamma_{2}}$ in the case of $\gamma_{1}=\gamma_{2}$ and $\tau>0 \ldots \ldots \ldots \ldots \ldots \ldots \ldots$

3.5 The dependence of $\mathcal{R}_{0}$ on $\frac{1}{\gamma_{1}}$ and $\frac{1}{\gamma_{2}}$ in the case of $\gamma_{1}=\gamma_{2}$ and $\tau=0 \ldots . . \quad 52$

3.6 Epidemic curves and attack rates of the two regions when $\mathcal{R}_{L, 1}>1$ and

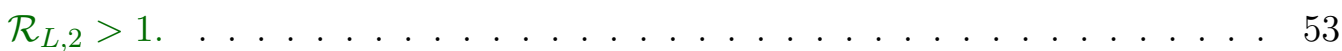

3.7 Epidemic curves and attack rates of the two regions when $\mathcal{R}_{L, 1}<1, \mathcal{R}_{L, 2}<1$

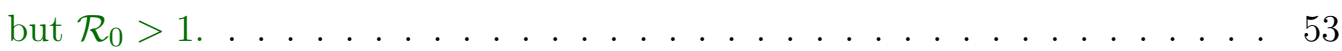

3.8 Epidemic curves of Canada and China. . . . . . . . . . . . . . . . 57

3.9 Epidemic curves of Canada and China with no disease transmission during travel. . . . . . . . . . . . . . . . . . . . . . . 58

3.10 Epidemic curves of Canada and the UK. . . . . . . . . . . . . . . . 59

3.11 Epidemic curves of Canada and Mexico. . . . . . . . . . . . . . . . . . . 61

3.12 Epidemic curves of Canada and Mexico with fitted peak times. . . . . . . . 62

4.1 Flow chart of disease transmission and travel dynamics of the SIRJ model. . 66

4.2 Flow chart of disease transmission and travel dynamics of the multiregional SIR model. . . . . . . . . . . . . . . . . . . . . . . . . 74

5.1 Schematic bifurcation diagrams. . . . . . . . . . . . . . . . . . . 85

5.2 Solutions of system $(5.6) \ldots \ldots \ldots \ldots$. . . . . . . . . . . 96

5.3 Bifurcation diagrams to illustrate Proposition 5.10 . . . . . . . . . . 97

5.4 Bifurcation diagrams to illustrate Propositions 5.12 and 5.13 . . . . . . . . 101 



\section{Chapter 1}

\section{Introduction}

Differential equations have been in the focus of mathematical biology since the famous work of Volterra. Dynamical models are powerful tools to describe various processes in life sciences by applying rigorous analytical techniques. Simple models are often unable to capture the rich variety of dynamics observed in biological phenomena, thus it is necessary to consider complex systems for the modeling. There are numerous reasons to incorporate time delays into biological models: they can represent resource regeneration times, maturation or incubation periods, reaction times, transport-related delays, or can simply account for the time required for a process to complete. The inclusion of delayed terms into differential equations brings us to the field of delay differential equations, where the derivative of the unknown function at a certain time is determined by the values of the function at previous times. The vast majority of works in the mathematical literature focus on model equations where the delayed feedback function is given explicitly (see, for instance, the Mackey-Glass equation, Nicholson's blowflies equation and Wright's equation), while others only require some particular properties (for instance, monotonicity or unimodularity) of the feedback. In this work, we propose various models from population dynamics and epidemiology where the delayed term in the corresponding system cannot be given explicitly by the model variables, but it depends on the solution of another differential system.

In this $\mathrm{Ph}$.D. dissertation, initial value problems for differential equations with such dynamically defined delayed feedback function will be considered. We propose a class of models from mathematical biology where the population is distributed over several discrete geographical regions, and show that the qualitative analysis of these models leads to the study of such systems if mobility (e.g., migration of species or transportation of individuals) between the regions is incorporated. Investigating the fundamental properties of the initial value problem is an interesting mathematical challenge in itself, but more significantly, the results obtained for the general system also enable us to gain more insight into the dynamics 
of the biological applications. In particular, we will describe the spatial spread of infectious diseases by formulating a series of mathematical models, each of which is equivalent to a large system of differential equations with dynamically defined delayed terms. First, we discuss a simple problem from mathematical biology.

\section{A basic model from population dynamics}

A simple model describing the growth of a single population with fixed period of temporary separation is given by

$$
\begin{aligned}
\dot{n}(t) & =b(n(t))-d(n(t))-q(n(t))+V(n(t-\tau)), \\
n_{0} & =\varphi
\end{aligned}
$$

where $t$ denotes time and functions $b, d$ and $q$ stand for recruitment, mortality and temporary separation (e.g., migration). Let $\tau>0$ be the fixed duration of separation. We define the phase space $C_{+}$as the nonnegative cone of $C([-\tau, 0], \mathbb{R})$, the Banach space of continuous functions from $[-\tau, 0]$ to $\mathbb{R}^{n}$ equipped with the supremum norm. The notation $n_{t}$ is used for the segment of the solution, where $n_{t} \in \mathcal{C}_{+}$and it holds that $n_{t}(\theta)=n(t-\theta)$ for $\theta \in[-\tau, 0]$. Then, $n_{0}=\varphi$ gives the initial state of the system for any $\varphi \in C_{+}$.

We say that a function $F: \mathbb{R} \rightarrow \mathbb{R}$ satisfies the Lipschitz condition on each bounded subset of $\mathbb{R}$ if for all $M>0$ there is a $K(M)>0$ such that the inequality $\left|F\left(x_{1}\right)-F\left(x_{2}\right)\right| \leq K\left|x_{1}-x_{2}\right|$ holds whenever $\left|x_{1}\right|,\left|x_{2}\right| \leq M$. We assume that $b, d, q: \mathbb{R} \rightarrow \mathbb{R}$ satisfy the Lipschitz property on each bounded subset of $\mathbb{R}$, which implies their continuity on $\mathbb{R}$. Since $b, d$ and $q$ denote the recruitment, mortality and separation functions, it should hold that they map nonnegative values to nonnegative values.

The function $V$ expresses the inflow of individuals arriving to the population at time $t$ after $\tau$ units of time of separation. For the precise definition of $V$, it is needed to describe the growth of the separated population. We assume that individuals who left the population due to separation in different times do not make contact to each other. Hence for each time $t_{*}$, the evolution of the density of the separated population with respect to the time elapsed since the beginning of separation is given by the following differential equation, when separation started at time $t_{*}$ :

$$
\begin{aligned}
\frac{\mathrm{d}}{\mathrm{d} \theta} m\left(\theta ; t_{*}\right) & =b^{S}\left(m\left(\theta ; t_{*}\right)\right)-d^{S}\left(m\left(\theta ; t_{*}\right)\right), \\
m\left(0 ; t_{*}\right) & =q\left(n\left(t_{*}\right)\right),
\end{aligned}
$$

where $\theta$ denotes the time elapsed since the beginning of separation, and functions $b^{S}$ and $d^{S}$ stand for recruitment and mortality during separation. At $\theta=0$, the density of the separated population is determined by the number of individuals who start separation at 
time $t_{*}$, hence the initial value for system (1.2) is given by $m\left(0 ; t_{*}\right)=q\left(n\left(t_{*}\right)\right)$. We assume that $b^{S}, d^{S}: \mathbb{R} \rightarrow \mathbb{R}$ satisfy the Lipschitz condition on each bounded subset of $\mathbb{R}$, which also yields that they are continuous on $\mathbb{R}$. The Picard-Lindelöf theorem ([22]) ensures that for any initial value $m_{*}$ there exists a unique solution $y\left(\theta ; 0, m_{*}\right)$ of system (1.2) on $[0, \alpha]$ for some $\alpha>0$. As separation lasts for $\tau$ units of time, it is reasonable to make the additional assumption that the unique solution exists at least for $\tau$ units of time for every $m_{*}$. This hypothesis can be fulfilled by some additional (however, biologically meaningful) conditions on $b^{S}$ and $d^{S}$; for instance, if the functions are defined such that the solution of (1.2) is bounded, then $y\left(\theta ; 0, m_{*}\right)$ can be continued for all positive times. In order to guarantee that nonnegative initial data give rise to nonnegative solutions of (1.2), we assume that the inequality $b^{S}(0)-d^{S}(0) \geq 0$ holds, and remark that this condition can be satisfied with several reasonable choices of the recruitment and mortality functions.

Now we are in the position to define the delayed feedback function $V$ in system (1.1). Since the duration of separation is exactly $\tau$ units of time, the inflow of individuals arriving to the population at $t_{*}+\tau$ after separation is determined by the solution of (1.2) at $\theta=\tau$ with $t_{*}$ fixed. We hence define the feedback function $V: \mathbb{R} \rightarrow \mathbb{R}$ as $V(v)=y(\tau ; 0, q(v))$.

By the formulation of the model, we obtain that the system describing the growth of the population is connected to another system via a delayed feedback term. More precisely, for the solution of system (1.1) at any time $t$, it is necessary to compute $V$ which is determined by the dynamics of system (1.2). Nevertheless, (1.2) takes the initial value from (1.1), and thus evaluating $V$ at $n(t-\tau)$ in system (1.1) results in a delayed term which cannot be given explicitly, but through the solution of the differential system (1.2). Henceforth, a system like (1.1) is referred to as a system of differential equations with dynamically defined delayed feedback term.

Chapter 2 of the dissertation is devoted to the formulation of an initial value problem for nonautonomous functional differential equations with dynamically defined delayed term. We investigate the fundamental properties of such systems. The general existence, uniqueness and continuous dependence result will be derived, and we give conditions for the nonnegativity of solutions. Furthermore, after determining the steady state solutions of the autonomous system, we obtain the linearized equation about the equilibria. Before proceeding to the general theory in Chapter 2, we present here a class of epidemic models to further motivate the study of such systems. The model proposed above with temporary separation will be revisited in Chapter 2 . 


\section{Epidemic models with travel-related infection}

National boundaries have never prevented infectious diseases from reaching distant territories; however, the speed at which an infectious agent can spread around the world via the global network of human transportation has significantly increased during the recent decades. Studying the role that the global airline transportation network plays in the worldwide spread of infectious diseases has been in the focus of mathematical epidemiology for a while, especially since the 2002-2003 SARS outbreak. Previous works on metapopulation models for disease spread in connected regions were mostly concerned only with the impact of the network structure and the volume of travel on the spatial dynamics (see Arino [2], Arino and van den Driessche [3], Baroyan et al. [5], Ruan et al. [36], Rvachev and Longini [37], Wang and Zhao [50] and the references therein). On the other hand, as it was highlighted in the risk assessment guideline of the European CDC [16], on-board transmission is a real threat for many infectious diseases, even during flights with a duration of less than eight hours.

The possibility that individuals may contract the disease while they travel was modeled by Cui, Liu, Takeuchi and Saito [10, 29, 42] using a system of ordinary differential equations based on the standard SIS (susceptible-infected-susceptible) epidemic model. While these works were based on the consideration that travel times between the regions are negligible, Liu et al. [28] incorporated the time needed to complete transportation into the model. Though the length of intercontinental flights is only a fraction of a day, it is reasonable to take into account any small delays if one's aim is to consider rapidly progressing diseases such as SARS and influenza. The SIS-type epidemic model proposed by Liu et al. [28] was further investigated by Nakata [33] and Nakata and Röst [34] by describing the global dynamics for an arbitrary $n$ number of regions with different characteristics and general travel networks.

The models in $[28,33,34]$ provide a good basis to investigate the spread of an infectious disease in regions which are connected by transportation. As a submodel, an age-structured system can be constructed to incorporate the possibility of disease transmission during travel, where age is the time elapsed since the start of the travel. Following the assumption that transmitting the infection is possible on-board, the model setup leads to a system of delay differential equations with delay representing travel time. The two systems, describing the dynamics in the regions and during transportation, are interconnected; initial values of the system for disease spread during travel depend on the state of the system in the regions, while the inflow term of arrivals to the regions after being in transportation for a fixed time arises as the solution of the subsystem for travel. If the 
subsystem can be solved analytically (this was the case for the age-structured SI model used in $[28,33,34])$, then the system for disease spread in the regions decouple from the subsystem. Recalling that initial data of the system during travel comes from the equations for the regions, the inflow term of travelers completing a trip appears as a delayed feedback term. On the other hand, in case of choosing SIR (susceptible-infected-recovered)-type models as an epidemic building block when the subsystem does not admit a closed form solution, the delayed term in the system for the regions cannot be expressed explicitly, but is defined dynamically, via the solutions of another system.

Three different epidemic models will be formulated in the thesis for the spread of infectious diseases in the population of individuals who travel between distant regions. In Chapter 3 we introduce an SEAIR (susceptible-exposed-asymptomatic infected-infectedrecovered)-based model to properly describe the temporal evolution of an epidemic in two regions of the world, which are connected by long distance travel such as intercontinental flights. We incorporate the consideration that infected travelers not only carry the disease from one place to another, but also infect some of their fellow passengers. This implies that the epidemic spreads more rapidly to farther regions than to closer ones, since longer flights provide more opportunity for infection. We use the phrase "antigravity model" to express this special feature of our model. This notion originates from the fact that the speed of disease propagation between regions is usually inversely proportional to the distance between those territories, as regions closer to each other are typically more connected; and this inverse relationship between speed and distance shows some analogy to physical gravity. Our model also distinguishes local residents from visitors to incorporate differences in individuals' mixing behavior. We use our framework to model the spread of influenza, one of the diseases that pose a threat of a global pandemic in modern times. For the numerical simulations which are performed to model three hypothetical situations with different characteristics for the regions, we use real demographic and air travel data. The model is also applied to the first wave of the A(H1N1) 2009 pandemic influenza in Mexico and Canada. One of the main challenges of this work is to compute the basic reproduction number $\left(\mathcal{R}_{0}\right)$, which gives the expected number of secondary infections generated by a single infected agent in a susceptible population. This quantity is of particular importance in epidemic models as it works as a threshold for the stability of the disease free steady state. To our knowledge, no general method has been published in the literature before to find $\mathcal{R}_{0}$ for the special type of systems considered in this Ph.D. dissertation.

The topic of investigating disease propagation in and between connected regions is further elaborated in Chapter 4. We present two SIR-based epidemic models, one for the 
spread of infection between two regions with an entry screening procedure initiated for travelers upon the arrival to a region, and another one with general incidence term in multiple regions. Setting up the system of differential equations for the first model leads to a situation when the dimension of the system for the disease spread in the regions differs from the dimension of the age-structured system during travel. The framework established in Chapter 2 for the general theory of systems with dynamically defined delayed feedback term is extended for the multiregional model to the case of multiple delays.

Chapter 5 is devoted to the bifurcation analysis of some epidemic models where traveling is considered. In particular, an SIVS (susceptible-infected-vaccinated-susceptible)based model will be investigated which exhibits the phenomenon of backward bifurcation, this is, there is an interval for $\mathcal{R}_{0}$ to the left of one, where the disease free equilibrium coexists with two positive fixed points, typically one stable and one unstable. Such behavior cannot be observed in the more common scenario of forward transcritical bifurcation, when positive steady states only exist for $\mathcal{R}_{0}>1$. Backward bifurcation and the presence of a stable endemic state for $\mathcal{R}_{0}<1$ have a significant epidemiological implication: the typical requirement of decreasing the reproduction number below one is no longer sufficient for effective epidemic control.

A number of mathematical models have been developed recently which deal with the various causes of backward bifurcation (see the well-known results of Dushoff, Huang, Castillo-Chavez, Hadeler and van den Driessche [15, 19, 20], and the work of Gumel [18] which provides a thorough overview of the relevant literature.). A simple disease transmission model with vaccination of susceptible individuals has been considered by Kribs-Zaleta and Velasco-Hernández in [26, 27], and later elaborated by Brauer in [7, 8], where one reason for the emergence of positive fixed points for $\mathcal{R}_{0}<1$ is that vaccination does not provide perfect protection against infection. We study an extension of this model as we investigate the effect of mobility on the dynamics by including the possibility of immigration of susceptible and vaccinated individuals into the model. After showing global stability results for the model, an explicit condition for the existence of backward bifurcation and multiple endemic equilibria will be given. We also examine in detail how the structure of the bifurcation diagram depends on the immigration. 


\section{Chapter 2}

\section{Differential equations with dynamically defined delay term}

The general form of systems of nonautonomous functional differential equations with dynamically defined delayed feedback function will be introduced. This work was motivated by applications from population dynamics and epidemiology where the model setup leads to a system of differential equations with the delay term defined via the solution of another system of differential equations. We obtain the general existence, uniqueness and continuous dependence result for the initial value problem by showing a Lipschitz property of the dynamically defined delayed feedback function, and give conditions for the nonnegativity of solutions. Steady-state solutions of the autonomous system will also be determined, and we derive the linearized equation about the equilibria. We revisit the single population model with fixed period of temporary separation, which has been introduced in Chapter 1 , and obtain some basic properties of the model.

\subsection{General formulation of the system}

We consider the initial value problem for the nonautonomous functional differential equation

$$
\begin{aligned}
x^{\prime}(t) & =\mathcal{F}\left(t, x_{t}\right), \\
x_{\sigma} & =\varphi,
\end{aligned}
$$

where $x: \mathbb{R} \rightarrow \mathbb{R}^{n}, n \in \mathbb{Z}_{+}, t, \sigma \in \mathbb{R}$ and $t \geq \sigma$. For $\tau>0$, we define our phase space $C=$ $C\left([-\tau, 0], \mathbb{R}^{n}\right)$ as the Banach space of continuous functions from $[-\tau, 0]$ to $\mathbb{R}^{n}$, equipped with the usual supremum norm $\|\cdot\|$. Let $\varphi \in C$ be the state of the system at $\sigma$. For the segments of solutions, we use the notation $x_{t} \in C$, where $x_{t}(\theta)=x(t+\theta)$ for $\theta \in[-\tau, 0]$. Let $\mathcal{F}: \mathbb{R} \times C \rightarrow \mathbb{R}^{n}$ and let $\mathcal{F}$ have the special form $\mathcal{F}(t, \phi)=f(t, \phi(0))+W(t, \phi(-\tau))$ for $\phi \in C, f: \mathbb{R} \times \mathbb{R}^{n} \rightarrow \mathbb{R}^{n}, W: \mathbb{R} \times \mathbb{R}^{n} \rightarrow \mathbb{R}^{n}$. 
In the sequel we use the notation $|v|_{j}$ for the Euclidean norm of any vector $v \in \mathbb{R}^{j}$ for $j \in \mathbb{Z}_{+}$. For $j=1$ we omit lower index 1 for simplicity. We define a Lipschitz condition as follows. For $j, l \in \mathbb{Z}_{+}$, we say that a function $F: \mathbb{R} \times \mathbb{R}^{j} \rightarrow \mathbb{R}^{l}$ satisfies the Lipschitz condition (Lip) on each bounded subset of $\mathbb{R} \times \mathbb{R}^{j}$ if:

(Lip) For all $a, b \in \mathbb{R}$ and $M>0$, there is a $K(a, b, M)>0$ such that:

$$
\left|F\left(t, x_{1}\right)-F\left(t, x_{2}\right)\right|_{l} \leq K\left|x_{1}-x_{2}\right|_{j}, a \leq t \leq b,\left|x_{1}\right|_{j},\left|x_{2}\right|_{j} \leq M
$$

We assume that $f: \mathbb{R} \times \mathbb{R}^{n} \rightarrow \mathbb{R}^{n}$ is continuous and satisfies (Lip) on each bounded subset of $\mathbb{R} \times \mathbb{R}^{n}$. For the definition of $W$, we make the following preparations. For any $s_{0} \in \mathbb{R}$ and $y_{*} \in \mathbb{R}^{m}, m \in \mathbb{Z}_{+}$, we consider the initial value problem

$$
\begin{aligned}
& y^{\prime}(s)=g(s, y(s)), \\
& y\left(s_{0}\right)=y_{*},
\end{aligned}
$$

where $y: \mathbb{R} \rightarrow \mathbb{R}^{m}, s, s_{0} \in \mathbb{R}, s \geq s_{0}, g: \mathbb{R} \times \mathbb{R}^{m} \rightarrow \mathbb{R}^{m}, g$ is continuous on $\mathbb{R} \times \mathbb{R}^{m}$ and satisfies the Lipschitz condition ( Lip) on each bounded subset of $\mathbb{R} \times \mathbb{R}^{m}$. The PicardLindelöf theorem (see Chapter II, Theorem 1.1 and Chapter V, Theorem 2.1 in [22]) states that, as $g$ is continuous on a parallelepiped $R: s_{0} \leq s \leq s_{0}+c,\left|y-y_{*}\right|_{m} \leq d$ with the bound $B$ for $|g|_{m}$ on $R$ and $g$ possesses the Lipschitz property (Lip), there exists a unique solution $y\left(s ; s_{0}, y_{*}\right)$ of $(2.2)$ on the interval $\left[s_{0}, s_{0}+\alpha\right]$ for $\alpha=\alpha_{s_{0}, y_{*}, c, d}:=\min \left\{c, \frac{d}{B}\right\}$, and the solution continuously depends on the initial data. We make the following additional assumption:

(*) For every $s_{0}$ and $y_{*}$, the solution $y\left(s ; s_{0}, y_{*}\right)$ of $(2.2)$ exists for $\tau$ units of time, i.e., on $\left[s_{0}, s_{0}+\tau\right]$.

Remark 2.1. The reader may notice that $(\star)$ is equivalent to the following assumption:

For every $s_{0}$ and $y_{*}$ the solution $y\left(s ; s_{0}, y_{*}\right)$ exists for all $s \geq s_{0}$.

Remark 2.2. With assuming various conditions on $g$ we can guarantee that $(\star)$ is fulfilled. For instance, for any $s_{0} \in \mathbb{R}$ and $L \in \mathbb{R}_{+}$, we define the constant $L_{g}=L_{g}\left(s_{0}, L\right)$ as the maximum of $|g|_{m}$ on the set $\left[s_{0}, s_{0}+\tau\right] \times\left\{v \in \mathbb{R}^{m}:|v|_{m} \leq 2 L\right\}$ (continuous functions attain their maximum on every compact set). Then the condition that for every $s_{0} \in \mathbb{R}$ and $L \in \mathbb{R}_{+}$the inequailty

$$
\tau \leq \frac{L}{L_{g}}
$$

holds immediately implies that $(\star)$ is satisfied. Indeed, for any $s_{0}$ and $y_{*}$, choose $c=\tau$, $d=\left|y_{*}\right|_{m}$. Then the Picard-Lindelöf theorem guarantees the existence and uniqueness of solution $y\left(s ; s_{0}, y_{*}\right)$ on $\left[s_{0}, s_{0}+\alpha\right]$ for $\alpha=\min \left\{\tau, \frac{\left|y_{*}\right|_{m}}{B}\right\}$, where $B$ is the bound for $|g|_{m}$ on the parallelepiped $s_{0} \leq s \leq s_{0}+\tau,\left|y-y_{*}\right|_{m} \leq\left|y_{*}\right|_{m}$. Choosing $L=\left|y_{*}\right|_{m}$, it 
follows from the definition of $L_{g}\left(s_{0}, L\right)$ that $B \leq L_{g}$ is satisfied, and using (2.3) we get $\tau \leq \frac{\left|y_{*}\right|_{m}}{L_{g}} \leq \frac{\left|y_{*}\right|_{m}}{B}$. We conclude that $\alpha=\tau$, hence the solution $y\left(s ; s_{0}, y_{*}\right)$ exists on $\left[s_{0}, s_{0}+\tau\right]$ and $(\star)$ is satisfied.

The less restrictive condition $\kappa:=\inf _{s_{0}, L} \frac{L}{L_{g}}>0$ implies the existence of the solution of $(2.2)$ on $\left[s_{0}, s_{0}+\kappa\right]$ for any $s_{0}$. Then it follows that for any $s_{0}$, the solution exists for all $s \geq s_{0}$, which is equivalent to $(\star)$. If we assume that a global Lipschitz condition ( $\mathrm{gLip}$ ) holds for $g$, that is, the Lipschitz constant for $g$ in ( Lip) can be chosen independently of $a, b$ and $M$, then for any $s_{0}$ and $y_{*}$ the solution of (2.2) exists for all $s \geq s_{0}$, thus also for $\tau$ units of time.

Now we are ready for the definition of $W$. For $h: \mathbb{R} \times \mathbb{R}^{n} \rightarrow \mathbb{R}^{m}, k: \mathbb{R} \times \mathbb{R}^{m} \rightarrow \mathbb{R}^{n}$, let us assume that $h$ and $k$ are continuous and satisfy the Lipschitz condition (Lip). For simplicity, we use the notation $y_{s_{0}, v}(s)=y\left(s ; s_{0}, h\left(s_{0}, v\right)\right)$ for the unique solution of system (2.2) in the case $y_{*}=h\left(s_{0}, v\right), v \in \mathbb{R}^{n}$. We define $W: \mathbb{R} \times \mathbb{R}^{n} \rightarrow \mathbb{R}^{n}$ as

$$
W(s, v)=k\left(s, y_{s-\tau, v}(s)\right)=k(s, y(s ; s-\tau, h(s-\tau, v))) .
$$

\subsection{Fundamental properties}

Our goal is to prove the usual existence and uniqueness theorem for (2.1). First we obtain the following simple results.

Proposition 2.3. $\mathcal{F}$ is continuous on $\mathbb{R} \times C$.

Proof. The Picard-Lindelöf theorem and $(\star)$ guarantee that for every $s_{0}, y_{*}$, there exists a unique solution of system $(2.2)$ on the interval $\left[s_{0}, s_{0}+\tau\right]$ and the solution $y\left(s ; s_{0}, y_{*}\right)$ continuously depends on the initial data. Moreover, $h$ and $k$ are continuous which implies the continuity of $W$. The function $f$ is also continuous, hence we conclude that $\mathcal{F}$ is continuous on $\mathbb{R} \times C$.

Proposition 2.4. For any $c, d \in \mathbb{R}$ such that $c<d$ and for any $L \in \mathbb{R}_{+}$, there exists $a$ bound $J=J(c, d, L)$ such that for any $s_{0} \in[c, d]$ and for any $y_{*} \in \mathbb{R}^{m}$ such that $\left|y_{*}\right|_{m} \leq L$, the inequality

$$
\left|y\left(s ; s_{0}, y_{*}\right)\right|_{m} \leq J
$$

holds for $s \in\left[s_{0}, s_{0}+\tau\right]$.

Proof. The Picard-Lindelöf theorem and $(\star)$ guarantee that for every $s_{0} \in \mathbb{R}$ and $y_{*} \in \mathbb{R}^{m}$, there exists a unique solution $y\left(s ; s_{0}, y_{*}\right)$ of system $(2.2)$ on the interval $\left[s_{0}, s_{0}+\tau\right]$, and the solution continuously depends on the initial data. Thus, for any $c, d \in \mathbb{R}$ where $c<d$ and for any $L \in \mathbb{R}_{+}$, the solution $y\left(s ; s_{0}, y_{*}\right)$, as a function of $s, s_{0}$ and $y_{*}$, is continuous 
on the set $\left\{\left(s_{1}, s_{2}, v\right): s_{1} \in[c, d+\tau], s_{2} \in[c, d], s_{1} \geq s_{2},|v|_{m} \leq L\right\}$. Continuous functions map compact sets to compact sets, hence there exists a constant $J(c, d, L)$ such that $\left|y\left(s ; s_{0}, y_{*}\right)\right|_{m} \leq J$. The proof is complete.

Now we show that besides continuity, $\mathcal{F}$ also satisfies a Lipschitz condition on each bounded subset of $\mathbb{R} \times C$ :

$\left(\operatorname{Lip}^{C}\right)$ For all $a, b \in \mathbb{R}$ and $M>0$, there is a $K(a, b, M)>0$ such that:

$$
|f(t, \phi)-f(t, \psi)|_{n} \leq K\|\phi-\psi\|, a \leq t \leq b,\|\phi\|,\|\psi\| \leq M
$$

Lemma 2.5. $\mathcal{F}$ satisfies the Lipschitz condition (Lip ${ }^{C}$ ) on each bounded subset of $\mathbb{R} \times C$.

Proof. Fix constants $a, b$ and $M, a<b, M>0$. Our aim is to find $K(a, b, M)$. Due to the continuity of $h$, there exists a constant $L_{h}(a, b, M)$ such that for any $\|\psi\| \leq M$ and $s_{0} \in[a-\tau, b-\tau]$, the inequality $\left|h\left(s_{0}, \psi(-\tau)\right)\right|_{m} \leq L_{h}$ holds. By choosing $c=a-\tau$, $d=b-\tau, L=L_{h}$ and $y_{*}=h\left(s_{0}, \psi(-\tau)\right)$ it follows from Proposition 2.4 that for any $s_{0} \in[a-\tau, b-\tau]$, the inequality $\left|y_{s_{0}, \psi(-\tau)}(s)\right|_{m} \leq J\left(a, b, L_{h}\right)$ is satisfied for $s \in\left[s_{0}, s_{0}+\tau\right]$.

Let $K_{h}=K_{h}(a, b, M)$ be the Lipschitz constant of $h$ on the set $[a-\tau, b-\tau] \times\{v \in$ $\left.\mathbb{R}^{n}:|v|_{n} \leq M\right\}$, let $K_{g}=K_{g}(a, b, M)$ be the Lipschitz constant of $g$ on the set $[a-\tau, b] \times$ $\left\{v \in \mathbb{R}^{m}:|v|_{m} \leq J\right\}$ (note that $J$ depends on $a, b$ and $M$ ). For any $\|\phi\|,\|\psi\| \leq M$ it holds that $|\phi(-\tau)|_{n},|\psi(-\tau)|_{n} \leq M$. Since the solution of (2.2) can be expressed as $y\left(s ; s_{0}, y_{*}\right)=y_{*}+\int_{s_{0}}^{s} g\left(r, y\left(r ; s_{0}, y_{*}\right)\right) d r$, for any $s_{0} \in[a-\tau, b-\tau]$ we have

$$
\begin{aligned}
\left|y_{s_{0}, \phi(-\tau)}(s)-y_{s_{0}, \psi(-\tau)}(s)\right|_{m}= & \mid h\left(s_{0}, \phi(-\tau)\right)+\int_{s_{0}}^{s} g\left(r, y_{s_{0}, \phi(-\tau)}(r)\right) \mathrm{d} r \\
& -\left.\left(h\left(s_{0}, \psi(-\tau)\right)+\int_{s_{0}}^{s} g\left(r, y_{s_{0}, \psi(-\tau)}(r)\right) \mathrm{d} r\right)\right|_{m} \\
\leq & \left|h\left(s_{0}, \phi(-\tau)\right)-h\left(s_{0}, \psi(-\tau)\right)\right|_{m} \\
& +\int_{s_{0}}^{s}\left|g\left(r, y_{s_{0}, \phi(-\tau)}(r)\right)-g\left(r, y_{s_{0}, \psi(-\tau)}(r)\right)\right|_{m} \mathrm{~d} r \\
\leq & K_{h}|| \phi-\psi \| \\
& +\int_{s_{0}}^{s} K_{g}\left|y_{s_{0}, \phi(-\tau)}(r)-y_{s_{0}, \psi(-\tau)}(r)\right|_{m} \mathrm{~d} r
\end{aligned}
$$

for $s \in\left[s_{0}, s_{0}+\tau\right]$. For a given $s_{0} \in[a-\tau, b-\tau]$ we define

$$
\Gamma(s)=\left|y_{s_{0}, \phi(-\tau)}(s)-y_{s_{0}, \psi(-\tau)}(s)\right|_{m}
$$

for $s \in\left[s_{0}, s_{0}+\tau\right]$. Then (2.5) gives

$$
\Gamma(s) \leq K_{h}\|\phi-\psi\|+K_{g} \int_{s_{0}}^{s} \Gamma(r) \mathrm{d} r,
$$


and from Gronwall's inequality we have that for any $s_{0} \in[a-\tau, b-\tau]$

$$
\Gamma(s) \leq K_{h}\|\phi-\psi\| e^{K_{g}\left(s-s_{0}\right)}
$$

holds for $s \in\left[s_{0}, s_{0}+\tau\right]$.

For any $t \in[a, b]$ it is satisfied that $t-\tau \in[a-\tau, b-\tau]$, hence for $s \in[t-\tau, t]$ we obtain

$$
\left|y_{t-\tau, \phi(-\tau)}(s)-y_{t-\tau, \psi(-\tau)}(s)\right|_{m} \leq K_{h}\|\phi-\psi\| e^{K_{g}(s-(t-\tau))}
$$

as a special case of (2.6) with $s_{0}=t-\tau$. The constant $J=J\left(a, b, L_{h}\right)$ was defined as the bound for $\left|y_{s_{0}, \psi(-\tau)}(s)\right|_{m}$ for any $s_{0} \in[a-\tau, b-\tau],\|\psi\| \leq M, s \in\left[s_{0}, s_{0}+\tau\right]$. For any $t \in[a, b]$ it follows that $t-\tau \in[a-\tau, b-\tau]$, hence the inequality $\left|y_{t-\tau, \psi(-\tau)}(t)\right|_{m} \leq J$ holds for any $\|\psi\| \leq M$. Let $K_{k}=K_{k}(a, b, M)$ be the Lipschitz constant of $k$ on the set $[a, b] \times\left\{v \in \mathbb{R}^{m}:|v|_{m} \leq J\right\}$. Then for any $t \in[a, b]$ and $\|\phi\|,\|\psi\| \leq M$ it is satisfied that $|\phi(-\tau)|_{n},|\psi(-\tau)|_{n} \leq M$, so we arrive to the following inequality:

$$
\begin{aligned}
|W(t, \phi(-\tau))-W(t, \psi(-\tau))|_{n} & =\left|k\left(t, y_{t-\tau, \phi(-\tau)}(t)\right)-k\left(t, y_{t-\tau, \psi(-\tau)}(t)\right)\right|_{n} \\
& \leq K_{k}\left|y_{t-\tau, \phi(-\tau)}(t)-y_{t-\tau, \psi(-\tau)}(t)\right|_{m} \\
& \leq K_{k} K_{h}|| \phi-\psi \| e^{K_{g} \tau}
\end{aligned}
$$

where we used (5.24) and (2.7).

Finally, let $K_{f}(a, b, M)$ be the Lipschitz constant of $f$ on the set $[a, b] \times\left\{v \in \mathbb{R}^{n}\right.$ : $\left.|v|_{n} \leq M\right\}$. Then for any $t \in[a, b]$ and for any $\|\phi\|,\|\psi\| \leq M$ it holds that $|\phi(0)|_{n}$, $|\psi(0)|_{n},|\phi(-\tau)|_{n},|\psi(-\tau)|_{n} \leq M$, hence we get

$$
\begin{aligned}
|\mathcal{F}(t, \phi)-\mathcal{F}(t, \psi)|_{n} & \leq|f(t, \phi(0))-f(t, \psi(0))|_{n}+|W(t, \phi(-\tau))-W(t, \psi(-\tau))|_{n} \\
& \leq K_{f}\|\phi-\psi\|+K_{k} K_{h}\|\phi-\psi\| e^{K_{g} \tau}
\end{aligned}
$$

and it is clear that $K_{f}(a, b, M)+K_{k}(a, b, M) K_{h}(a, b, M) e^{\tau K_{g}(a, b, M)}$ is a suitable choice for $K(a, b, M)$, the Lipschitz constant of $\mathcal{F}$ on the set $[a, b] \times\{\psi \in C:\|\psi\| \leq M\}$.

We state the following simple remark.

Remark 2.6. If $f, g, h$ and $k$ satisfy a global Lipschitz condition ( $g$ Lip), that is, if $K_{f}$, $K_{g}, K_{h}$ and $K_{k}$ can be chosen independently of $a, b$ and $M$ in the definition of the Lipschitz condition $($ Lip $)$, then a global Lipschitz condition $\left(\right.$ gLip $\left.^{C}\right)$ holds for $\mathcal{F}$, i.e., there exists a Lipschitz constant $K$ of $\mathcal{F}$ which is independent of $a, b$ and $M$. 
Now, as we have proved that $\mathcal{F}$ is continuous and satisfies the Lipschitz condition $\left(\mathrm{Lip}^{C}\right)$, all conditions of Theorem 3.7 in [38] are satisfied. We arrive to the following result.

Theorem 2.7. Let $\sigma \in \mathbb{R}, M>0$. There exists $A>0$, depending only on $M$ such that if $\phi \in C=C\left([-\tau, 0], \mathbb{R}^{n}\right)$ satisfies $\|\phi\| \leq M$, then there exists a unique solution $x(t)=x(t ; \sigma, \phi)$ of (2.1), defined on $[\sigma-\tau, \sigma+A]$. In addition, if $K$ is the Lipschitz constant for $\mathcal{F}$ corresponding to $[\sigma, \sigma+A]$ and $M$, then

$$
\max _{\sigma-\tau \leq \eta \leq \sigma+A}|x(\eta ; \sigma, \phi)-x(\eta ; \sigma, \psi)|_{n} \leq\|\phi-\psi\| e^{K A} \text { for any }\|\phi\|,\|\psi\| \leq M .
$$

Assuming stronger conditions on $f, g, h$ and $k$, we arrive to a more general existence result. We follow Remark 3.8 in [38].

Remark 2.8. If $f, g, h$ and $k$ satisfy condition ( $g$ Lip $)$, then condition $\left(g L i p^{C}\right)$ arises for $\mathcal{F}$ and we do not need to make any restrictions on $A$ in Theorem 2.7. More precisely, its statements hold for any $A>0$. In this case, the solution exists for every $t \geq \sigma$ and the inequality

$$
\left\|x_{t}(\phi)-x_{t}(\psi)\right\| \leq\|\phi-\psi\| e^{K(t-\sigma)}
$$

holds for all $t \geq \sigma$.

Most functional differential equations that arise in population dynamics or epidemiology deal only with nonnegative quantities. Therefore it is important to see what conditions ensure that nonnegative initial data give rise to nonnegative solutions.

We reformulate (2.1) using the definition of $\mathcal{F}$. Since $\mathcal{F}\left(t, x_{t}\right)=f(t, x(t))+W(t, x(t-\tau))$, we consider the following system of differential equations, which is equivalent to (2.1):

$$
\begin{aligned}
x^{\prime}(t) & =f(t, x(t))+W(t, x(t-\tau)), \\
x_{\sigma} & =\varphi .
\end{aligned}
$$

We claim that under reasonable assumptions, the solution of system (2.8) preserves nonnegativity for nonnegative initial data. Let us suppose that for each $t \in \mathbb{R}, h$ and $k$ map nonnegative vectors to nonnegative vectors. We also assume that for every $i \in\{1, \ldots, n\}$, $j \in\{1, \ldots, m\}, u \in \mathbb{R}_{+}^{n}, w \in \mathbb{R}_{+}^{m}$ and $t, s \in \mathbb{R}, u_{i}=0$ implies $f_{i}(t, u) \geq 0$ and $w_{j}=0$ implies $g_{j}(s, w) \geq 0$. Then for any nonnegative initial value, the solution of system (2.2) is nonnegative, which implies that for every $i \in\{1, \ldots, n\}, v \in \mathbb{R}_{+}^{n}$ and $t \in \mathbb{R}$, the inequality $(k(t, y(t ; t-\tau, h(t-\tau, v))))_{i}=W_{i}(t, v) \geq 0$ holds. Hence $f_{i}(t, u)+W_{i}(t, v) \geq 0$ is satisfied for $u, v \in \mathbb{R}_{+}^{n}, u_{i}=0, t \in \mathbb{R}$, all conditions of Theorem 3.4 in [38] hold and we conclude that nonnegative initial data give rise to nonnegative solutions of system (2.8). Clearly systems (2.8) and (2.1) are equivalent, which implies that the result automatically holds for system (2.1). We summarize our assumptions and their consequence. 
Proposition 2.9. Suppose that $h: \mathbb{R} \times \mathbb{R}^{n} \rightarrow \mathbb{R}^{m}$ and $k: \mathbb{R} \times \mathbb{R}^{m} \rightarrow \mathbb{R}^{n}$ map nonnegative vectors to nonnegative vectors for each $t \in \mathbb{R}$, moreover assume that

$$
\begin{gathered}
\forall i, t, \forall u \in \mathbb{R}_{+}^{n}: u_{i}=0 \Rightarrow f_{i}(t, u) \geq 0, \\
\forall j, s, \forall w \in \mathbb{R}_{+}^{m}: w_{j}=0 \Rightarrow g_{j}(s, w) \geq 0 .
\end{gathered}
$$

Then for nonnegative initial data the solution of system (2.1) preserves nonnegativity, i.e., $x(t) \geq 0$ for all $t \geq \sigma$ where it is defined.

\subsection{The autonomous case}

\subsubsection{Basic properties}

As a special case of system (2.1), we may derive similar results for the autonomous system. Let $x: \mathbb{R} \rightarrow \mathbb{R}^{n}, y: \mathbb{R} \rightarrow \mathbb{R}^{m}, t, s \in \mathbb{R}$, let $f: \mathbb{R}^{n} \rightarrow \mathbb{R}^{n}, g: \mathbb{R}^{m} \rightarrow \mathbb{R}^{m}, h: \mathbb{R}^{n} \rightarrow \mathbb{R}^{m}$, $k: \mathbb{R}^{m} \rightarrow \mathbb{R}^{n}$. Let us assume that $f, g, h$ and $k$ satisfy the Lipschitz condition (Lip), which can be stated as follows. For $j, l \in \mathbb{Z}_{+}$, we say that a function $F: \mathbb{R}^{j} \rightarrow \mathbb{R}^{l}$ satisfies the Lipschitz condition (Lip) if for all $M>0$ there is a $K(M)>0$ such that for $\left|x_{1}\right|_{j},\left|x_{2}\right|_{j} \leq M$ the inequality $\left|F\left(x_{1}\right)-F\left(x_{2}\right)\right|_{l} \leq K\left|x_{1}-x_{2}\right|_{j}$ holds. There is no need to assume the continuity for $f, g, h$ and $k$, since these functions are independent of $t$ and hence this property follows from the Lipschitz condition ( Lip). For $\tau>0$, let $C=C\left([-\tau, 0], \mathbb{R}^{n}\right)$ be the phase space, where $C$ has been defined in Section 2.1. Then system (2.1) has the form

$$
\begin{aligned}
x^{\prime}(t) & =\mathcal{F}\left(x_{t}\right), \\
x_{0} & =\varphi,
\end{aligned}
$$

where $t \geq 0, \varphi \in C$ is the state of the system at $t=0, \mathcal{F}: C \rightarrow \mathbb{R}^{n}$ and $\mathcal{F}$ has the special form $\mathcal{F}(\phi)=f(\phi(0))+W(\phi(-\tau)), \phi \in C$. For any $y_{*} \in \mathbb{R}^{m}$, system (2.2) turns into

$$
\begin{aligned}
y^{\prime}(s) & =g(y(s)), \\
y(0) & =y_{*},
\end{aligned}
$$

where $s \geq 0$. Similarly as in Section 2.1, the Picard-Lindelöf theorem guarantees the existence and uniqueness of the solution of system $(2.10)$ on $[0, \alpha]$ for some $\alpha>0$. We make the following additional assumption:

$(\star \star)$ For every $y_{*}$, the solution $y\left(s ; 0, y_{*}\right)$ of $(2.10)$ exists at least for $\tau$ units of time.

This is equivalent to the assumption that $y\left(s ; 0, y_{*}\right)$ exists on $[0, \infty)$ for every $y_{*}$, which holds if $g$ satisfies ( $g$ Lip) (see Remark 2.2). We use the notation $y_{0, v}(s)=y(s ; 0, h(v)$ ) for the unique solution of system (2.10) in the case $y_{*}=h(v)$, and we define $W: \mathbb{R}^{n} \rightarrow \mathbb{R}^{n}$ by

$$
W(v)=k\left(y_{0, v}(\tau)\right)=k(y(\tau ; 0, h(v)))
$$


where $v \in \mathbb{R}^{n}$. It is straightforward that the Lipschitz condition $\left(\operatorname{Lip}^{C}\right)$ and the continuity of $\mathcal{F}$ hold, furthermore if we assume that $f, g, h$ and $k$ satisfy the global Lipschitz condition $(g L i p)$, then we obtain that condition $\left(g L i p{ }^{C}\right)$ is satisfied for $\mathcal{F}$ (for the definitions of ( $g$ Lip) and $\left(\mathrm{gLip}^{C}\right)$, see Remark 2.6). As an immediate consequence of Theorem 2.7, we state the following corollary.

Corollary 2.10. Suppose that $M>0$. There exists $A>0$, depending only on $M$ such that if $\phi \in C$ satisfies $\|\phi\| \leq M$, then there exists a unique solution $x(t)=x(t ; 0, \phi)$ of (2.9), defined on $[-\tau, A]$. In addition, if $K$ is the Lipschitz constant for $\mathcal{F}$ corresponding to $M$, then

$$
\max _{-\tau \leq \eta \leq A}|x(\eta ; 0, \phi)-x(\eta ; 0, \psi)|_{n} \leq\|\phi-\psi\| e^{K A} \text { for any }\|\phi\|,\|\psi\| \leq M .
$$

The following remark arises automatically as the autonomous case of Remark 2.8.

Remark 2.11. If $f, g, h$ and $k$ satisfy the global Lipschitz condition ( $g$ Lip), then we do not need to make any restrictions on $A$ in Corollary 2.10. More precisely, its statements hold for all $A>0$. In this case, the solution exists for all $t \geq 0$ and the inequality

$$
\left\|x_{t}(\phi)-x_{t}(\psi)\right\| \leq\|\phi-\psi\| e^{K t}
$$

holds for all $t \geq 0$.

Clearly we can adapt Proposition 2.9 to the autonomous system with similar conditions.

Corollary 2.12. Suppose that $h: \mathbb{R}^{n} \rightarrow \mathbb{R}^{m}$ and $k: \mathbb{R}^{m} \rightarrow \mathbb{R}^{n}$ map nonnegative vectors to nonnegative vectors, moreover assume that

$$
\begin{gathered}
\forall i, \forall u \in \mathbb{R}_{+}^{n}: u_{i}=0 \Rightarrow f_{i}(u) \geq 0, \\
\forall j, \forall w \in \mathbb{R}_{+}^{m}: w_{j}=0 \Rightarrow g_{j}(w) \geq 0 .
\end{gathered}
$$

Then for nonnegative initial data the solution of system (2.9) preserves nonnegativity, i.e., $x(t) \geq 0$ for all $t \geq 0$ where it is defined.

\subsubsection{Equilibria and linearization}

Consider the nonlinear functional differential equation system (2.9)

$$
x^{\prime}(t)=\mathcal{F}\left(x_{t}\right),
$$

where $\mathcal{F}(\phi)=f(\phi(0))+W(\phi(-\tau))$ for $\phi \in C$. Then $x(t)=\bar{x} \in \mathbb{R}^{n}$ is a steady-state solution of (2.9) if and only if $\mathcal{F}(\hat{\bar{x}})=0$ holds, where $\hat{\bar{x}} \in C$ is the constant function equal to $\bar{x}$. Suppose there exists such an equilibrium. We formulate the linearized system about the equilibrium $\bar{x}$ as

$$
z^{\prime}(t)=D \mathcal{F}(\hat{\bar{x}}) z_{t}
$$


where $D \mathcal{F}(\hat{\bar{x}}): C \rightarrow \mathbb{R}^{n}$ is a bounded linear operator and $z: \mathbb{R} \rightarrow \mathbb{R}^{n}$. Due to the special form of $\mathcal{F},(2.12)$ reformulates as

$$
z^{\prime}(t)=A_{1} z(t)+A_{2} z(t-\tau)
$$

where $A_{1}=D f(\bar{x}) \in \mathbb{R}^{n \times n}$ and $A_{2}=D W(\bar{x}) \in \mathbb{R}^{n \times n}$.

Proposition 2.13. Suppose that $g, h$ and $k$ are continuously differentiable. Then the matrix $D W(\bar{x})$ can be represented with $g, h$ and $k$ as follows:

$$
D W(\bar{x})=D k(y(\tau ; 0, h(\bar{x}))) e^{\int_{0}^{\tau} D g(y(r ; 0, h(\bar{x}))) \mathrm{d} r} D h(\bar{x})
$$

Proof. Theorem 3.3 in Chapter I in [21] states that as $g$ has continuous first derivative, the solution $y\left(s ; 0, y_{*}\right)$ of system $(2.10)$ is continuously differentiable with respect to $s$ and $y_{*}$ on its domain of definition. The matrix $\frac{\partial y\left(s ; 0, y_{*}\right)}{\partial y_{*}} \in \mathbb{R}^{m \times m}$ satisfies the linear variational equation

$$
Y^{\prime}(s)=D g\left(y\left(s ; 0, y_{*}\right)\right) Y(s)
$$

where $Y: \mathbb{R} \rightarrow \mathbb{R}^{m \times m}$ (we use slightly different notations from [21]) and $\frac{\partial y\left(0 ; 0, y_{*}\right)}{\partial y_{*}}=I$, where $I$ denotes the identity. As from (2.13) it follows that $Y(s)=e^{\int_{0}^{s} D g\left(y\left(r ; 0, y_{*}\right)\right) \mathrm{d} r} Y(0)$, for $Y(0)=I$ we conclude that

$$
\frac{\partial y\left(\tau ; 0, y_{*}\right)}{\partial y_{*}}=e^{\int_{0}^{\tau} D g\left(y\left(r ; 0, y_{*}\right)\right) \mathrm{d} r}
$$

holds for $s=\tau$. From (2.11) we get that for $v \in \mathbb{R}^{n}$,

$$
\begin{aligned}
D W(v) & =D k(y(\tau ; 0, h(v))) \frac{\partial y(\tau ; 0, h(v))}{\partial v} \\
& =D k(y(\tau ; 0, h(v))) \frac{\partial y(\tau ; 0, h(v))}{\partial y_{*}} D h(v),
\end{aligned}
$$

hence from (2.14) and (2.15) we derive

$$
D W(v)=D k(y(\tau ; 0, h(v))) e^{\int_{0}^{\tau} D g(y(r ; 0, h(v))) \mathrm{d} r} D h(v) .
$$

Finally, setting $v=\bar{x}$ in (2.16), we arrive to the equality

$$
D W(\bar{x})=D k(y(\tau ; 0, h(\bar{x}))) e^{\int_{0}^{\tau} D g(y(r ; 0, h(\bar{x}))) \mathrm{d} r} D h(\bar{x}) .
$$

Note that $D k(y(\tau ; 0, h(\bar{x}))) \in \mathbb{R}^{n \times m}, D g(y(r ; 0, h(\bar{x}))) \in \mathbb{R}^{m \times m}$ and $D h(\bar{x}) \in \mathbb{R}^{m \times n}$, hence the result of the matrix multiplication is indeed $D W(\bar{x}) \in \mathbb{R}^{n \times n}$. The proof is complete.

It follows from (2.11) that $\bar{x}$ satisfies the equation $-f(\bar{x})=k(y(\tau ; 0, h(\bar{x})))$. However, $\bar{x}$ being a steady-state solution of (2.9) does not necessarily imply that $y(s, 0 ; h(\bar{x}))=h(\bar{x})$ 
holds for $s \in[0, \tau]$, i.e., $h(\bar{x})$ is an equilibrium of $(2.10)$.

We say that $\bar{x} \in \mathbb{R}^{n}$ is a total equilibrium of systems (2.9) and (2.10) if $x(t)=\bar{x}$ is a steady-state solution of (2.9) and $y(s)=h(\bar{x})$ is a steady-state solution of (2.10). The equilibrium solution $y(s)=\bar{y}, \bar{y} \in \mathbb{R}^{m}$ of $(2.10)$ satisfies the equation $g(\bar{y})=0$, and since $h(\bar{x})=\bar{y}$ and $-f(\bar{x})=k(y(\tau ; 0, h(\bar{x})))$ should hold for the total equilibrium, we conclude that $\bar{x}$ arises as the solution of the system

$$
\begin{aligned}
-f(\bar{x}) & =k(h(\bar{x})), \\
g(h(\bar{x})) & =0 .
\end{aligned}
$$

It follows from (2.17) that, in the special case when $f$ and $g$ are invertible functions, the total equilibrium can be expressed as $\bar{x}=f^{-1}\left(-k\left(g^{-1}(0)\right)\right)$, moreover we also obtain $\bar{y}=h\left(f^{-1}\left(-k\left(g^{-1}(0)\right)\right)\right)$.

We remark that if the functions $g, h$ and $k$ are continuously differentiable and $\bar{x}$ is the total equilibrium of systems (2.9) and (2.10), then it follows from Proposition 2.13 that the matrix $D W(\bar{x})$ has the form $D W(\bar{x})=D k(h(\bar{x})) e^{\tau D g(h(\bar{x}))} D h(\bar{x})$.

\subsection{A model from population dynamics with temporary sep- aration}

We are now in the position to revisit the single population model described by system (1.1) in the introduction, and obtain some basic properties for the model. First, for a given $t_{*}$ we define $y(s)=m\left(s ; t_{*}\right)$ and let $g(y)=b^{S}(y)-d^{S}(y)$, where $y:[0, \tau] \rightarrow \mathbb{R}, g: \mathbb{R} \rightarrow \mathbb{R}$. Then (2.10) is a compact form of the autonomous system (1.2). Furthermore, for $h, k: \mathbb{R} \rightarrow \mathbb{R}$ we let $h(v)=q(v), k(v)=v$, moreover we define $x:[0, \infty) \rightarrow \mathbb{R}$ as $x(t)=n(t)$ and $f: \mathbb{R} \rightarrow \mathbb{R}$ as $f(x)=b(x)-d(x)-q(x)$. Then it follows that system (1.1) can be written in a closed form as the autonomous system (2.9).

Clearly, the functions $f, g, h$ and $k$ defined above satisfy the Lipschitz condition (Lip) on each bounded subset of $\mathbb{R}$, moreover $(\star \star)$ also holds by means of the assumption on the solution of system (1.2). Hence $\mathcal{F}$, defined as $\mathcal{F}(\phi)=f(\phi(0))+W(\phi(-\tau))$ for $\phi \in C_{+}$ satisfies the Lipschitz condition $\left(\operatorname{Lip}^{C}\right)$, so Corollary 2.10 states that system (1.1) has a unique solution defined on $[-\tau, A]$ for some $A>0$. By assuming that condition ( $g$ Lip) holds for $b, d, q, b^{S}$ and $d^{S}$, we get that $f, g, h$ and $k$ satisfy the global Lipschitz condition ( Lip) and $A=\infty$. We have assumed in the model setup that $q=h$ maps nonnegative values to nonnegative values, which obviously holds for $k$ as well, moreover we gave the condition $b^{S}(0)-d^{S}(0) \geq 0$. In addition, if we suppose that $b(0)-d(0)-q(0) \geq 0$ is satisfied (e.g., $b(0)=d(0)=q(0)=0$ holds in many models), then Corollary 2.12 implies 
that for nonnegative initial data the solution of system (1.1) preserves nonnegativity, that is, $C_{+}$is invariant. We summarize these results in the following theorem.

Theorem 2.14. System (1.1) has a unique nonnegative solution for nonnegative initial values. Biologically reasonable conditions on the functions $b, d, q, b^{S}$ and $d^{S}$ ensure that the solution exists on $[-\tau, \infty)$. 


\section{Chapter 3}

\section{A delay model for the spread of pandemics between connected regions}

National boundaries have never prevented infectious diseases from reaching distant territories. However, the speed at which an infectious agent can spread around the world via the global airline transportation network has significantly increased during recent decades. We introduce an SEAIR (susceptible-exposed-asymptomatic infected-infected-recovered)based model to investigate the spread of an infectious disease in two regions which are connected by transportation. As a submodel, an age-structured system is constructed to incorporate the possibility of disease transmission during travel, where age is the time elapsed since the start of the travel. We show that the model is equivalent to a large system of autonomous differential equations of the form considered in Chapter 2. After describing fundamental, but biologically relevant properties of the system, we detail the calculation of the basic reproduction number and obtain disease transmission dynamics results in terms of $\mathcal{R}_{0}$. We parametrize our model for influenza, and use real demographic and air travel data for the numerical simulations. To understand the role of the different characteristics of the regions in the propagation of the disease, three distinct origin-destination pairs will be considered. The model will also be fitted to the first wave of the influenza A(H1N1) 2009 pandemic in Mexico and Canada.

\subsection{Introduction}

The global network of human transportation has been playing a paramount role in the spatial spread of infectious diseases. The high connectedness of distant territories by air travel makes it possible for a disease to invade regions far away from the source faster than 
ever. Some infectious diseases, such as tuberculosis, measles and seasonal influenza, have been known to be transmissible during commercial flights. The importance of the global air travel network was highlighted in the 2002-2003 SARS outbreak (WHO [51]), and clearly contributed to the global spread of the 2009 pandemic influenza A(H1N1) (Khan et al. [25]). Therefore, mathematically describing the spread of infectious diseases on the global human transportation network is of critical public health importance.

There are a few well-known studies which constructed and analyzed various metapopulation models for disease spread in connecting regions (see Arino [2], Arino and van den Driessche [3], Baroyan et al. [5], Ruan et al. [36], Rvachev and Longini [37], Wang and Zhao [50] and the references therein). These studies focus mainly on the impact of spatial dispersal of infected individuals from one region to another, and do not consider transportation as a platform of disease dynamics. However, during long distance travel, such as intercontinental flights, a single infected individual may infect several other passengers during the flight (Wagner et al. [49], Technical Report of ECDC [16]), thus potentially inducing multiple generating infections in the destination region. It is therefore desirable to properly describe the spread of the disease via long distance travel, that incorporates into the models the transmission dynamics during the travel.

Cui et al. [10] and Takeuchi et al. [42] modeled the possibility that individuals may contract the disease while traveling by a system of ordinary differential equations based on the standard SIS epidemic model. They discovered that the disease can persist in regions connected by human transportation even if the infection died out in all regions in the absence of travel. Liu et al. [28] noted that the previously proposed models [10, 29, 42] implicitly used the assumption that the transportation between regions occurs instantaneously. For some diseases of major public health concern, such as SARS and influenza, the progress of the disease is so fast that even a short delay (a fraction of a day) can be significant. Based on such considerations, Liu et al. [28] introduced the time needed to complete the travel into the SIS-type epidemic model and also the possible infections during this time. Nakata [33] described the global dynamics of this system for two identical regions in terms of the basic reproduction number. The model was later generalized by Nakata and Röst [34] to the case of $n$ regions with different characteristics and arbitrary travel networks.

The purpose of this chapter is to formulate a model to properly describe the temporal evolution of an epidemics in regions connected by long distance travel, such as intercontinental flights. The European Centre for Disease Prevention and Control (ECDC) developed 
a risk assessment guideline [16] for infectious diseases transmitted on aircrafts. Existing studies confirmed that on-board transmission was possible in flights even with a duration of less than eight hours. For most diseases which pose a threat of a global pandemic, an SIStype model is not adequate. For this reason, here we use the SEAIR (susceptible-exposedasymptomatic infected-infected-recovered) model as a basic epidemic model building block in the regions and also during travel. The SIS model can be reduced to a logistic equation and then can be solved analytically. This property was heavily used in the analysis done in $[28,33,34]$. However, the lack of closed form solution causes substantial technical difficulties in the analysis of SEAIR-type models, as will be shown in this paper.

More significantly, the aforementioned existing models did not distinguish local residents from temporary visitors in the model setup. In reality, the large part of travels are return trips, and not only the number of visitors, but also the average time that visitors spend in the other region may significantly affect the speed of spatial spread of the disease. If visitors spend more time in a region which is a hotspot of the disease, they will more likely carry the disease back to their region of origin. In addition, visitors and local residents may have very different contact rates and mixing patterns, for example if the visitors are typically on holiday and stay in selected resorts and hotels. Hence in our model we use different compartments for residents and visitors to capture this phenomenon.

Many multiregional epidemic models, specially the gravity type models, are based on the assumption that the speed of the spread of epidemics between regions is inversely proportional to the distance between those regions (see for example Tuite et al. [45] for the recent cholera outbreak in Haiti). However, in case of air travel, the travel behavior is different and can be just the opposite. First, the number of travelers does not depend directly on the distance between regions, but determined by other, more important factors, such as business and cultural relations, tourist attractions. Second, the transmission rate of an infectious disease can be much higher than usual when a large number of passengers are sharing the same cabin, and the longer the flight (which means the distance is larger between regions), the greater number of infections can be expected (Wagner et al. [49]). Hence air travel models we are proposing here is in principle "antigravity".

\subsection{Model description}

We formulate a dynamical model describing the spread of an infectious disease in and between two regions, and also during travel from one region to the other. We divide the entire 


\begin{tabular}{|c|c|}
\hline \multicolumn{2}{|r|}{ Variables } \\
\hline$F_{j}^{r}$ & Force of infection of residents in region $j$ \\
\hline$F_{j}^{v}$ & Force of infection of visitors in region $j$ \\
\hline$F_{j, k}^{T}$ & Force of infection during travel from region $j$ to region $k$ \\
\hline$S_{j}^{r}, E_{j}^{r}, A_{j}^{r}, I_{j}^{r}, R_{j}^{r}$ & $\begin{array}{l}\text { Susceptible, exposed, asymptomatic, symptomatic } \\
\text { infected and recovered residents in region } j\end{array}$ \\
\hline$S_{j}^{v}, E_{j}^{v}, A_{j}^{v}, I_{j}^{v}, R_{j}^{v}$ & $\begin{array}{l}\text { Susceptible, exposed, asymptomatic, symptomatic } \\
\text { infected and recovered visitors in region } j\end{array}$ \\
\hline$N_{j}^{r}, N_{j}^{v}, N_{j}$ & $\begin{array}{l}\text { Total population size of residents, visitors and } \\
\text { all individuals in region } j\end{array}$ \\
\hline$s_{j, k}^{r}, e_{j, k}^{r}, a_{j, k}^{r}, i_{j, k}^{r}, r_{j, k}^{r}$ & $\begin{array}{l}\text { Density of susceptible, exposed, asymptomatic, } \\
\text { symptomatic infected and recovered individuals } \\
\text { during the travel from } j \text { to } k \text { (traveling to visit } k \text { ) }\end{array}$ \\
\hline$s_{j, k}^{v}, e_{j, k}^{v}, a_{j, k}^{v}, i_{j, k}^{v}, r_{j, k}^{v}$ & $\begin{array}{l}\text { Density of susceptible, exposed, asymptomatic, } \\
\text { symptomatic infected and recovered individuals during } \\
\text { the travel from } j \text { to } k \text { (returning to } k \text { from visiting } j \text { ) }\end{array}$ \\
\hline$n_{j, k}^{r}, n_{j, k}^{v}, n_{j, k}$ & $\begin{array}{l}\text { Total density of residents, visitors and } \\
\text { all individuals during the travel from } j \text { to } k\end{array}$ \\
\hline
\end{tabular}

Table 3.1: Variables of the SEAIR model $(j, k \in\{1,2\}, j \neq k)$. In the table, "density" means the density with respect to the time elapsed since the start of travel.

populations of the two regions into the disjoint classes $S_{j}^{m}, E_{j}^{m}, A_{j}^{m}, I_{j}^{m}, R_{j}^{m}, j \in\{1,2\}$, $m \in\{r, v\}$, where the letters $S, E, A, I$ and $R$ represent the compartments of susceptible, exposed, asymptomatic infected, symptomatic infected and recovered individuals, respectively. Lower index $j \in\{1,2\}$ specifies the current region, upper index $m \in\{r, v\}$ denotes the residential status of the individual in the current region (resident versus visitor). For instance, $S_{1}^{v}$ is the compartment of individuals who are susceptible for the disease and staying in region 1 as a visitor (hence, they originally belong to region 2), members of $A_{2}^{r}$ are those who are asymptomatic infected residents in region 2.

Let $S_{j}^{m}(t), E_{j}^{m}(t), A_{j}^{m}(t), I_{j}^{m}(t), R_{j}^{m}(t), j \in\{1,2\}, m \in\{r, v\}$, be the number of individuals belonging to $S_{j}^{m}, E_{j}^{m}, A_{j}^{m}, I_{j}^{m}, R_{j}^{m}$, respectively, at time $t$. The transmission rate between an infected individual with residential status $m$ and a susceptible individual with residential status $n$ in region $j(j \in\{1,2\}, m, n \in\{r, v\})$ is denoted by $\beta_{j}^{m, n}$. Let $F_{j}^{r}$ denote the force of infection (this is, the rate at which susceptible individuals acquire the disease) of residents, and $F_{j}^{v}$ the force of infection of visitors in region $j$. Model parameter $\mu_{E}$ denotes the inverse of the incubation period, $\mu_{A}$ and $\mu_{I}$ are the recovery 


\begin{tabular}{|c|c|}
\hline \multicolumn{2}{|r|}{ Key model parameters } \\
\hline$\Lambda_{j}$ & Recruitment rate in region $j$ \\
\hline$d_{j}^{r}, d_{j}^{v}$ & Natural death rate of residents and visitors of region $j$ \\
\hline$\delta$ & Disease-induced death rate \\
\hline$\beta_{j}^{m, n}$ & Transmission rate between an infected individual \\
\hline & with residential status ' $m$ ' and a susceptible individual \\
\hline & with residential status ' $n$ ' in region $j(m, n \in\{r, v\})$ \\
\hline$\beta^{T}$ & Transmission rate during the travel \\
\hline$\alpha_{j}$ & Traveling rate of residents of region $j$ to region $k$ \\
\hline$\gamma_{j}$ & Inverse of duration of visitors' stay in region $j$ \\
\hline$\tau$ & Duration of travel between the regions \\
\hline$p$ & Probability of developing symptoms \\
\hline$\rho$ & Reduction of infectiousness of asymptotic infecteds \\
\hline$\mu_{E}, \mu_{E}^{T}$ & $\begin{array}{l}\text { Reciprocal of the length of the incubation period } \\
\text { in the regions and during the travel }\end{array}$ \\
\hline$\mu_{A}, \mu_{A}^{T}$ & Recovery rate of asymptomatic infecteds \\
\hline & in the regions and during the travel \\
\hline$\mu_{I}, \mu_{I}^{T}$ & Recovery rate of symptomatic infecteds \\
\hline & in the regions and during the travel \\
\hline
\end{tabular}

Table 3.2: Parameters of the SEAIR model $(j, k \in\{1,2\}, j \neq k)$.

rates of asymptomatic and symptomatic infected individuals. Let $\rho$ be the reduction factor of infectiousness of asymptomatic infected individuals (we assume they are capable of transmitting the disease, but generally with a lower rate than symptomatic infected individuals). Let $p$ denote the probability that an infected individual develops symptoms, and let $\delta$ denote disease-induced mortality rate. We assume constant recruitment terms $\Lambda_{j}$, while $d_{j}^{r}$ and $d_{j}^{v}$ denote natural mortality rate of residents and visitors in region $j$. We denote the travel rate of residents between region $j$ and region $k$ by $\alpha_{j}$ and the rate visitors of region $j$ travel back to region $k$ by $\gamma_{j}$, thus $1 / \gamma_{j}$ is the average time visitors spend in region $j$. For the total population of residents, visitors, and all individuals currently being in region $j$ at time $t$, we use the notations

$$
\begin{aligned}
N_{j}^{r}(t) & =S_{j}^{r}(t)+E_{j}^{r}(t)+A_{j}^{r}(t)+I_{j}^{r}(t)+R_{j}^{r}(t), \\
N_{j}^{v}(t) & =S_{j}^{v}(t)+E_{j}^{v}(t)+A_{j}^{v}(t)+I_{j}^{v}(t)+R_{j}^{v}(t), \\
N_{j}(t) & =N_{j}^{r}(t)+N_{j}^{v}(t) .
\end{aligned}
$$

We divide the population during travel into the classes $s_{j, k}^{m}, e_{j, k}^{m}, a_{j, k}^{m}, i_{j, k}^{m}, r_{j, k}^{m}$. Letters $s, e, a, i, r$ denote susceptible, exposed, asymptomatic infected, symptomatically infected 
Region 1

Region 2

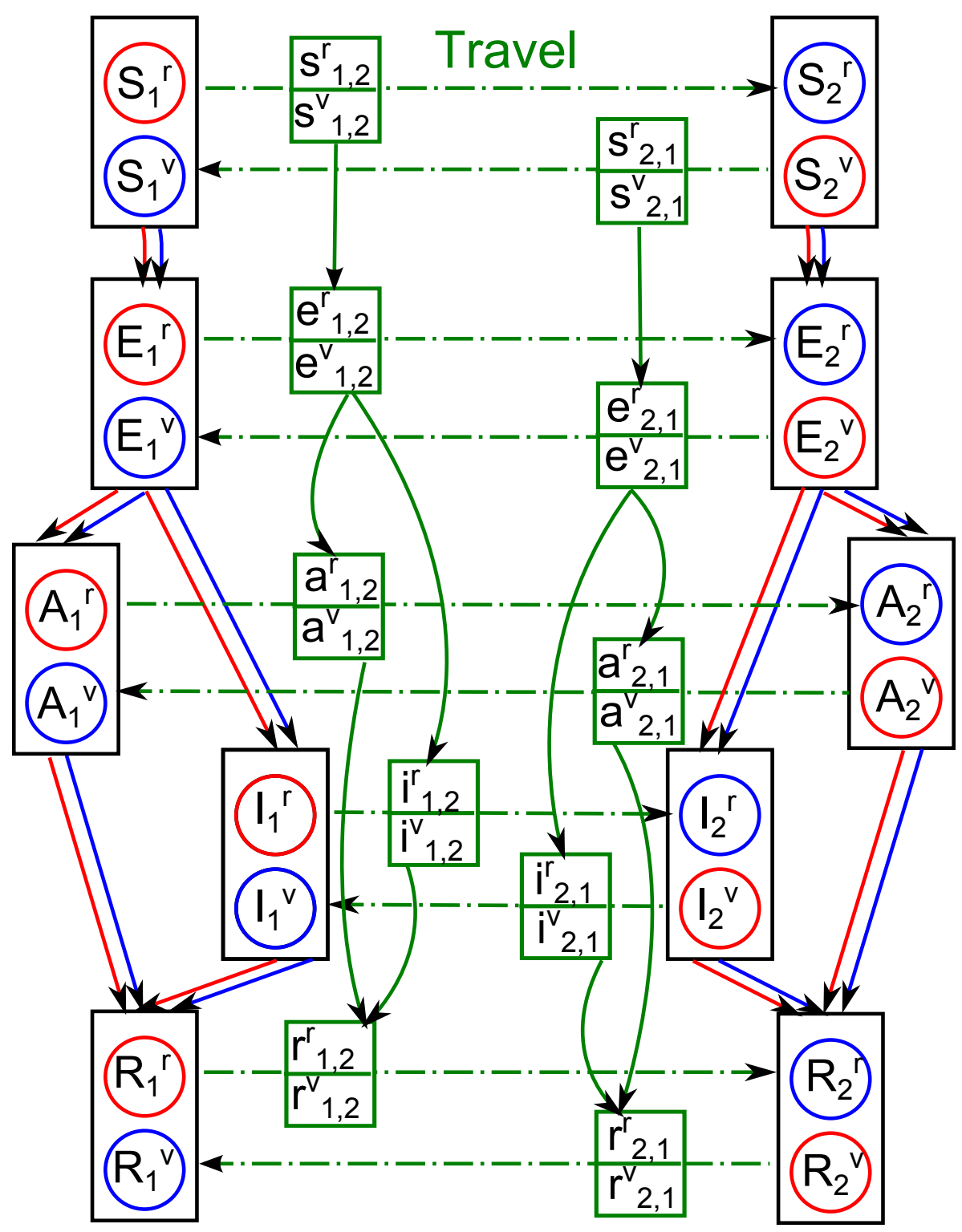

Figure 3.1: Color-coded flow chart of disease transmission and travel dynamics of the SEAIR model. The disease transmission in the two regions is shown in two different columns, the disease progresses vertically from the top to the bottom. Classes having the same origins are marked by the same colors. Red corresponds to the classes originated from region 1 , blue represents classes of region 2. Arrows colored with the same colors indicate how the disease progresses. Green dashed-dotted arrows represent traveling. Green solid arrows show the dynamics of the pandemic during the course of the travel. The description of the variables can be found in Table 3.1 . 
and recovered travelers, respectively. Lower indices $j, k \in\{1,2\}, j \neq k$, indicate that individuals are traveling from region $j$ to region $k$. Upper index $m \in\{r, v\}$ determines individuals' residential status in the region they have just left: for instance, an individual who is now being in $r_{1,2}^{v}$ is recovered, traveling from region 1 to region 2 , was a visitor in region 1 , which means the individual originally belongs to region 2 .

Let $\tau>0$ denote the average time required to complete a one-way trip. To describe the disease dynamics during travel, we define $s_{j, k}^{m}\left(\theta ; t_{*}\right), e_{j, k}^{m}\left(\theta ; t_{*}\right), a_{j, k}^{m}\left(\theta ; t_{*}\right), i_{j, k}^{m}\left(\theta ; t_{*}\right)$, $r_{j, k}^{m}\left(\theta ; t_{*}\right), j, k \in\{1,2\}, j \neq k, m \in\{r, v\}$, as the density of individuals who started travel at time $t_{*}$ and belong to classes $s_{j, k}^{m}, e_{j, k}^{m}, a_{j, k}^{m}, i_{j, k}^{m}, r_{j, k}^{m}$ with respect to $\theta$, where $\theta \in[0, \tau]$ denotes the time elapsed since the beginning of the travel. Let

$$
n_{j, k}^{m}\left(\theta ; t_{*}\right)=s_{j, k}^{m}\left(\theta ; t_{*}\right)+e_{j, k}^{m}\left(\theta ; t_{*}\right)+a_{j, k}^{m}\left(\theta ; t_{*}\right)+i_{j, k}^{m}\left(\theta ; t_{*}\right)+r_{j, k}^{m}\left(\theta ; t_{*}\right),
$$

where $j, k \in\{1,2\}, j \neq k, m \in\{r, v\}$, and let

$$
n_{j, k}\left(\theta ; t_{*}\right)=n_{j, k}^{r}\left(\theta ; t_{*}\right)+n_{j, k}^{v}\left(\theta ; t_{*}\right)
$$

Thus, $\int_{\theta_{2}}^{\theta_{1}} n_{j, k}(\theta ; t-\theta) \mathrm{d} \theta$ is the number of individuals who left region $j$ in the time interval $\left[t-\theta_{1}, t-\theta_{2}\right]$, where $\tau \geq \theta_{1} \geq \theta_{2} \geq 0$. In particular, for $\theta_{1}=\tau$ and $\theta_{2}=0$, this gives the total number of individuals who are in transition from region $j$ to region $k$ at time $t$. We assume that infected individuals do not die during travel, hence $n_{j, k}\left(\theta ; t_{*}\right)=n_{j, k}\left(0 ; t_{*}\right)$ for all $\theta \in[0, \tau]$. During the course of travel, infected individuals can transmit the disease at the rate $\beta^{T}$. We use the notations $\mu_{E}^{T}, \mu_{A}^{T}, \mu_{I}^{T}$ for the inverse of the incubation period and the recovery rates of asymptomatic and symptomatic infected individuals during travel. Let $F_{j, k}^{T}$ denote the force of infection during travel from region $j$ to region $k$. Then $s_{j, k}^{m}(\tau ; t-\tau), e_{j, k}^{m}(\tau ; t-\tau), a_{j, k}^{m}(\tau ; t-\tau), i_{j, k}^{m}(\tau ; t-\tau), r_{j, k}^{m}(\tau ; t-\tau)$ gives the inflow of individuals arriving from region $j$ to compartments $S_{k}^{n}, E_{k}^{n}, A_{k}^{n}, I_{k}^{n}, R_{k}^{n}, j, k \in\{1,2\}$, $j \neq k, m, n \in\{r, v\}, m \neq n$, respectively, at time $t$.

All variables and model parameters are listed in Tables 3.1 and 3.2. The flow chart of the model is depicted in Figure 3.1. Based on the assumptions formulated above, we obtain the following system of differential equations for the disease transmission in the regions: 


$$
\left\{\begin{array}{l}
\dot{S}_{j}^{r}(t)=\Lambda_{j}-S_{j}^{r}(t) F_{j}^{r}(t)-\left(d_{j}^{r}+\alpha_{j}\right) S_{j}^{r}(t)+s_{k, j}^{v}(\tau ; t-\tau), \\
\dot{E}_{j}^{r}(t)=S_{j}^{r}(t) F_{j}^{r}(t)-\left(d_{j}^{r}+\mu_{E}+\alpha_{j}\right) E_{j}^{r}(t)+e_{k, j}^{v}(\tau ; t-\tau), \\
\dot{A}_{j}^{r}(t)=(1-p) \mu_{E} E_{j}^{r}(t)-\left(d_{j}^{r}+\alpha_{j}+\mu_{A}\right) A_{j}^{r}(t)+a_{k, j}^{v}(\tau ; t-\tau), \\
\dot{I}_{j}^{r}(t)=p \mu_{E} E_{j}^{r}(t)-\left(d_{j}^{r}+\alpha_{j}+\delta+\mu_{I}\right) I_{j}^{r}(t)+i_{k, j}^{v}(\tau ; t-\tau), \\
\dot{R}_{j}^{r}(t)=\mu_{I} I_{j}^{r}(t)+\mu_{A} A_{j}^{r}(t)-\left(d_{j}^{r}+\alpha_{j}\right) R_{j}^{r}(t)+r_{k, j}^{v}(\tau ; t-\tau), \\
\dot{S}_{j}^{v}(t)=-S_{j}^{v}(t) F_{j}^{v}(t)-\left(d_{j}^{v}+\gamma_{j}\right) S_{j}^{v}(t)+s_{k, j}^{r}(\tau ; t-\tau), \\
\dot{E}_{j}^{v}(t)=S_{j}^{v}(t) F_{j}^{v}(t)-\left(d_{j}^{v}+\mu_{E}+\gamma_{j}\right) E_{j}^{v}(t)+e_{k, j}^{r}(\tau ; t-\tau), \\
\dot{A}_{j}^{v}(t)=(1-p) \mu_{E} E_{j}^{v}(t)-\left(d_{j}^{v}+\gamma_{j}+\mu_{A}\right) A_{j}^{v}(t)+a_{k, j}^{r}(\tau ; t-\tau), \\
\dot{I}_{j}^{v}(t)=p \mu_{E} E_{j}^{v}(t)-\left(d_{j}^{v}+\gamma_{j}+\delta+\mu_{I}\right) I_{j}^{v}(t)+i_{k, j}^{r}(\tau ; t-\tau), \\
\dot{R}_{j}^{v}(t)=\mu_{I} I_{j}^{v}(t)+\mu_{A} A_{j}^{v}(t)-\left(d_{j}^{v}+\gamma_{j}\right) R_{j}^{v}(t)+r_{k, j}^{r}(\tau ; t-\tau),
\end{array}\right.
$$

where

$$
\begin{aligned}
& F_{j}^{r}(t)=\frac{1}{N_{j}(t)}\left(\beta_{j}^{r r}\left(I_{j}^{r}(t)+\rho A_{j}^{r}(t)\right)+\beta_{j}^{v r}\left(I_{j}^{v}(t)+\rho A_{j}^{v}(t)\right)\right), \\
& F_{j}^{v}(t)=\frac{1}{N_{j}(t)}\left(\beta_{j}^{r v}\left(I_{j}^{r}(t)+\rho A_{j}^{r}(t)\right)+\beta_{j}^{v v}\left(I_{j}^{v}(t)+\rho A_{j}^{v}(t)\right)\right) .
\end{aligned}
$$

For each given $t_{*}$, the following system $(T)$ describes the evolution of the densities during the travel initiated at time $t_{*}$.

$$
\left\{\begin{array}{l}
\frac{\mathrm{d}}{\mathrm{d} \theta} s_{j, k}^{r}\left(\theta ; t_{*}\right)=-s_{j, k}^{r}\left(\theta ; t_{*}\right) F_{j, k}^{T}\left(\theta ; t_{*}\right), \\
\frac{\mathrm{d}}{\mathrm{d} \theta} e_{j, k}^{r}\left(\theta ; t_{*}\right)=s_{j, k}^{r}\left(\theta ; t_{*}\right) F_{j, k}^{T}\left(\theta ; t_{*}\right)-\mu_{E}^{T} e_{j, k}^{r}\left(\theta ; t_{*}\right), \\
\frac{\mathrm{d}}{\mathrm{d} \theta} a_{j, k}^{r}\left(\theta ; t_{*}\right)=(1-p) \mu_{E}^{T} e_{j, k}^{r}\left(\theta ; t_{*}\right)-\mu_{A}^{T} a_{j, k}^{r}\left(\theta ; t_{*}\right), \\
\frac{\mathrm{d}}{\mathrm{d} \theta} i_{j, k}^{r}\left(\theta ; t_{*}\right)=p \mu_{E}^{T} e_{j, k}^{r}\left(\theta ; t_{*}\right)-\mu_{I}^{T} i_{j, k}^{r}\left(\theta ; t_{*}\right), \\
\frac{\mathrm{d}}{\mathrm{d} \theta} r_{j, k}^{r}\left(\theta ; t_{*}\right)=\mu_{A}^{T} a_{j, k}^{r}\left(\theta ; t_{*}\right)+\mu_{I}^{T} i_{j, k}^{r}\left(\theta ; t_{*}\right) \\
\frac{\mathrm{d}}{\mathrm{d} \theta} s_{j, k}^{v}\left(\theta ; t_{*}\right)=-s_{j, k}^{v}\left(\theta ; t_{*}\right) F_{j, k}^{T}\left(\theta ; t_{*}\right) \\
\frac{\mathrm{d}}{\mathrm{d} \theta} e_{j, k}^{v}\left(\theta ; t_{*}\right)=s_{j, k}^{v}\left(\theta ; t_{*}\right) F_{j, k}^{T}\left(\theta ; t_{*}\right)-\mu_{E}^{T} e_{j, k}^{v}\left(\theta ; t_{*}\right), \\
\frac{\mathrm{d}}{\mathrm{d} \theta} a_{j, k}^{v}\left(\theta ; t_{*}\right)=(1-p) \mu_{E}^{T} e_{j, k}^{v}\left(\theta ; t_{*}\right)-\mu_{A}^{T} a_{j, k}^{v}\left(\theta ; t_{*}\right) \\
\frac{\mathrm{d}}{\mathrm{d} \theta} i_{j, k}^{v}\left(\theta ; t_{*}\right)=p \mu_{E}^{T} e_{j, k}^{v}\left(\theta ; t_{*}\right)-\mu_{I}^{T} i_{j, k}^{v}\left(\theta ; t_{*}\right) \\
\frac{\mathrm{d}}{\mathrm{d} \theta} r_{j, k}^{v}\left(\theta ; t_{*}\right)=\mu_{A}^{T} a_{j, k}^{v}\left(\theta ; t_{*}\right)+\mu_{I}^{T} i_{j, k}^{v}\left(\theta ; t_{*}\right)
\end{array}\right.
$$


where $j, k \in\{1,2\}, j \neq k$, and

$$
\begin{aligned}
F_{j, k}^{T}\left(\theta ; t_{*}\right)= & \frac{\beta^{T}}{n_{j, k}\left(\theta ; t_{*}\right)}\left(i_{j, k}^{r}\left(\theta ; t_{*}\right)+i_{j, k}^{v}\left(\theta ; t_{*}\right)+\rho\left(a_{j, k}^{r}\left(\theta ; t_{*}\right)+a_{j, k}^{v}\left(\theta ; t_{*}\right)\right)\right), \\
n_{j, k}\left(\theta ; t_{*}\right)= & \alpha_{j}\left(S_{j}^{r}\left(t_{*}\right)+E_{j}^{r}\left(t_{*}\right)+A_{j}^{r}\left(t_{*}\right)+I_{j}^{r}\left(t_{*}\right)+R_{j}^{r}\left(t_{*}\right)\right) \\
& +\gamma_{j}\left(S_{j}^{v}\left(t_{*}\right)+E_{j}^{v}\left(t_{*}\right)+A_{j}^{v}\left(t_{*}\right)+I_{j}^{v}\left(t_{*}\right)+R_{j}^{v}\left(t_{*}\right)\right) \\
= & \alpha_{j} N_{j}^{r}\left(t_{*}\right)+\gamma_{j} N_{j}^{v}\left(t_{*}\right) .
\end{aligned}
$$

For $\theta=0$, the densities are determined by the rates at which individuals start their travels from one region to the other at time $t_{*}$. Hence, the initial values for system $(T)$ at $\theta=0$ are given by

$$
\left\{\begin{array}{cl}
s_{j, k}^{r}\left(0 ; t_{*}\right)=\alpha_{j} S_{j}^{r}\left(t_{*}\right), & s_{j, k}^{v}\left(0 ; t_{*}\right)=\gamma_{j} S_{j}^{v}\left(t_{*}\right), \\
e_{j, k}^{r}\left(0 ; t_{*}\right)=\alpha_{j} E_{j}^{r}\left(t_{*}\right), & e_{j, k}^{v}\left(0 ; t_{*}\right)=\gamma_{j} E_{j}^{v}\left(t_{*}\right), \\
a_{j, k}^{r}\left(0 ; t_{*}\right)=\alpha_{j} A_{j}^{r}\left(t_{*}\right), & a_{j, k}^{v}\left(0 ; t_{*}\right)=\gamma_{j} A_{j}^{v}\left(t_{*}\right), \\
i_{j, k}^{r}\left(0 ; t_{*}\right)=\alpha_{j} I_{j}^{r}\left(t_{*}\right), & i_{j, k}^{v}\left(0 ; t_{*}\right)=\gamma_{j} I_{j}^{v}\left(t_{*}\right), \\
r_{j, k}^{r}\left(0 ; t_{*}\right)=\alpha_{j} R_{j}^{r}\left(t_{*}\right), & r_{j, k}^{v}\left(0 ; t_{*}\right)=\gamma_{j} R_{j}^{v}\left(t_{*}\right)
\end{array}\right.
$$

for $j, k \in\{1,2\}, j \neq k$.

Now we turn our attention to the terms $s_{j, k}^{m}(\tau ; t-\tau), e_{j, k}^{m}(\tau ; t-\tau), a_{j, k}^{m}(\tau ; t-\tau)$, $i_{j, k}^{m}(\tau ; t-\tau), r_{j, k}^{m}(\tau ; t-\tau)$ in system $(L)$, which are the densities of individuals arriving to classes $S_{k}^{n}, E_{k}^{n}, A_{k}^{n}, I_{k}^{n}, R_{k}^{n}, j, k \in\{1,2\}, j \neq k, m, n \in\{r, v\}, m \neq n$, respectively, at time $t$ upon completing a one-way trip from region $j$. At time $t$, these terms are determined by the solution of system $(T)$ with initial values $(I V T)$ for $t_{*}=t-\tau$ at $\theta=\tau$ :

(i) individuals who enter region $k$ at time $t$ are those who left region $j$ at time $t-\tau$;

(ii) residents of region $j$ become visitors of region $k$ and vice versa $(m \neq n)$ upon completing a one-way trip;

(iii) an individual may move to a different compartment during travel, for example a susceptible resident who travels from region $j$ may arrive as an infected visitor to region $k(j, k \in\{1,2\}, j \neq k)$, as given by the dynamics of system $(T)$.

Next we specify initial values for system $(L)$ at $t=0$. Since travel takes $\tau$ units of time to complete, arrivals to region $j$ are determined by the state of region $k(j, k \in\{1,2\}$, $j \neq k)$ at $t-\tau$, via the solution of systems $(T)$ and $(I V T)$. Thus, we set up initial functions as follows: 


$$
\left\{\begin{aligned}
& S_{j}^{r}(u)=\varphi_{S, j}^{r}(u), S_{j}^{v}(u)=\varphi_{S, j}^{v}(u), \\
& E_{j}^{r}(u)=\varphi_{E, j}^{r}(u), E_{j}^{v}(u)=\varphi_{E, j}^{v}(u), \\
& A_{j}^{r}(u)=\varphi_{A, j}^{r}(u), \quad A_{j}^{v}(u)=\varphi_{A, j}^{v}(u), \\
& I_{j}^{r}(u)=\varphi_{I, j}^{r}(u), \quad I_{j}^{v}(u)=\varphi_{I, j}^{v}(u), \\
& R_{j}^{r}(u)=\varphi_{R, j}^{r}(u), \quad R_{j}^{v}(u)=\varphi_{R, j}^{v}(u),
\end{aligned}\right.
$$

where $u \in[-\tau, 0]$, and each $\varphi_{K, j}^{m}$ is a continuous function for $j \in\{1,2\}, m \in\{r, v\}, K \in$ $\{S, E, A, I, R\}$.

Note that systems $(L)$ and $(T)$ are interconnected, in order to determine the dynamics of the model, simultaneous solution of them is required. Considering the fact that disease transmission is possible during travel, the solution of system $(T)$ at $(\tau ; t-\tau)$ is required for all $t \geq 0$ to find the solution of $(L)$. However, in order to obtain the solution of $(T)$ at $(\tau ; t-\tau)$, it is necessary to use the solution of $(L)$ at $t-\tau$, because $(T)$ takes the initial conditions from $(L)$. Hence, in order to describe the disease transmission in the regions, the solution of another differential equation system is required at each time $t$, which has initial values depending on the earlier state of the system on the regions. Thus $(L)$ is a delay differential system, where the delayed feedback is determined by a solution of a parallel system of ordinary differential equations. In previous papers with travel delay, such as $[28,33,34]$, the authors used an SIS type system during travel, which was analytically solvable, thus it was possible to express the delayed feedback explicitly. Unlike the SIS model, the SEAIR model is not analytically solvable, therefore here we have to deal with a system of functional differential equations, where the delay term is given only implicitly via a solution of a nonlinear system of ordinary differential equations.

\subsection{Basic properties of the model}

In this section, we show that our model is equivalent to a system of nonlinear functional differential equations where the delay term is defined dynamically, via the solution of another system of differential equations. Then we also investigate some biologically relevant properties of the system. Let

$$
X_{j}^{r}=\left(\begin{array}{c}
S_{j}^{r} \\
E_{j}^{r} \\
A_{j}^{r} \\
I_{j}^{r} \\
R_{j}^{r}
\end{array}\right), X_{j}^{v}=\left(\begin{array}{c}
S_{j}^{v} \\
E_{j}^{v} \\
A_{j}^{v} \\
I_{j}^{v} \\
R_{j}^{v}
\end{array}\right), y_{j, k}^{r}=\left(\begin{array}{c}
s_{j, k}^{r} \\
e_{j, k}^{r} \\
a_{j, k}^{r} \\
i_{j, k}^{r} \\
r_{j, k}^{r}
\end{array}\right), y_{j, k}^{v}=\left(\begin{array}{c}
s_{j, k}^{v} \\
e_{j, k}^{v} \\
a_{j, k}^{v} \\
i_{j, k}^{v} \\
r_{j, k}^{v}
\end{array}\right),
$$


where $j, k \in\{1,2\}, j \neq k$, then for

$$
x=\left(\begin{array}{c}
X_{1}^{r} \\
X_{1}^{v} \\
X_{2}^{r} \\
X_{2}^{v}
\end{array}\right), y=\left(\begin{array}{c}
y_{2,1}^{v} \\
y_{2,1}^{r} \\
y_{1,2}^{v} \\
y_{1,2}^{r}
\end{array}\right)
$$

it holds that $x: \mathbb{R}_{+} \rightarrow \mathbb{R}^{20}$ and $y: \mathbb{R}_{+} \times \mathbb{R}_{+} \rightarrow \mathbb{R}^{20}$. For a given $t_{*} \in \mathbb{R}_{+}$, we define the system $\left(T^{*}\right)$ for $y(\cdot)=y\left(\cdot ; t_{*}\right)$ as

$$
\begin{aligned}
y^{\prime}(\theta) & =g(y(\theta)), \\
y(0) & =y_{0},
\end{aligned}
$$

where $\theta \in \mathbb{R}_{+}, y_{0} \in \mathbb{R}^{20}, g: \mathbb{R}^{20} \rightarrow \mathbb{R}^{20}$, and $g_{i}$ equals the right-hand side of the equation for $y_{i}$ in system $(T)$ for each $i \in\{1, \ldots, 20\}$; for instance,

$$
g_{7}(y)=\beta^{T} \frac{y_{6}}{\sum_{j=1}^{10} y_{j}}\left(y_{4}+y_{9}+\rho\left(y_{3}+y_{8}\right)\right)-\mu_{E}^{T} y_{7} .
$$

Let $y\left(\theta ; t_{*}, y_{0}\right)$ denote the solution of the initial value problem $\left(T^{*}\right)$, defined for $t_{*}$. For now let us assume that the solution exists and is unique for $\theta \in[0, \tau]$ for each $y_{0}$, and we will shortly detail the proof of this statement. By introducing

$$
h_{i}(v)= \begin{cases}\gamma_{2} v_{i+15} & \text { if } i=1, \ldots, 5 \\ \alpha_{2} v_{i+5} & \text { if } i=6, \ldots, 10 \\ \gamma_{1} v_{i-5} & \text { if } i=11, \ldots, 15 \\ \alpha_{1} v_{i-15} & \text { if } i=16, \ldots, 20\end{cases}
$$

we get that $\left(T^{*}\right)$ is a compact form of $(T)$ and $(I V T)$ with $y_{0}=h\left(x\left(t_{*}\right)\right)$.

Furthermore, we define $f, f: \mathbb{R}^{20} \rightarrow \mathbb{R}^{20}$, where for each $i \in\{1, \ldots, 20\}, f_{i}$ is given by the right-hand side of the equation of $x_{i}$ in $(L)$ without the inflow from travel; for instance,

$$
f_{16}(x)=-\frac{x_{16}}{\sum_{j=11}^{20} x_{j}}\left(\beta_{2}^{r v}\left(x_{14}+\rho x_{13}\right)+\beta_{2}^{v v}\left(x_{19}+\rho x_{18}\right)\right)-\left(d_{2}^{v}+\gamma_{2}\right) x_{16} .
$$

Finally we let $W(v)=y(\tau ; t-\tau, h(v)), W: \mathbb{R}^{20} \rightarrow \mathbb{R}^{20}$, and claim that our system $(L)$ can be written in a closed form as a system of functional differential equations

$$
\begin{aligned}
x^{\prime}(t) & =\mathcal{F}\left(x_{t}\right), \\
x_{0} & =\Phi,
\end{aligned}
$$

where $\mathcal{F}\left(x_{t}\right)=f(x(t))+W(x(t-\tau)), \mathcal{F}: C_{+} \rightarrow \mathbb{R}^{20}$, with the phase space defined as the nonnegative cone $C_{+}=C\left([-\tau, 0], \mathbb{R}_{+}^{20}\right)$ of the Banach space of continuous functions from $[-\tau, 0]$ to $\mathbb{R}^{20}$, equipped with the supremum norm. Using the notations of 
$(I V L)$, we also let $\Phi=\left(\Phi_{1}^{r}, \Phi_{1}^{v}, \Phi_{2}^{r}, \Phi_{2}^{v}\right)^{T}$, where $\Phi_{j}^{r}=\left(\varphi_{S, j}^{r}, \varphi_{E, j}^{r}, \varphi_{A, j}^{r}, \varphi_{I, j}^{r}, \varphi_{R, j}^{r}\right)^{T}, \Phi_{j}^{v}=$ $\left(\varphi_{S, j}^{v}, \varphi_{E, j}^{v}, \varphi_{A, j}^{v}, \varphi_{I, j}^{v}, \varphi_{R, j}^{v}\right)^{T}, j \in\{1,2\}$.

Fundamental properties for functional differential equations with dynamically defined delayed feedback term (elaborated in Chapter 2) guarantee that there exists a unique solution of the model equations. Both $\left(L^{*}\right)$ and $\left(T^{*}\right)$ are autonomous, thus we refer to Section 2.3 of Chapter 2 to get that the general existence and uniqueness result holds for $\left(L^{*}\right)$ if $\mathcal{F}$ satisfies a global Lipschitz property $\left(g \operatorname{Lip}^{C}\right)$, in which case the solution exists on $[0, \infty)$. In terms of the notations of Chapter 2 , for $n=20, m=20$ and $k: \mathbb{R}^{20} \rightarrow \mathbb{R}^{20}, k(v)=v$, systems $\left(L^{*}\right)$ and $\left(T^{*}\right)$ can be obtained in compact forms as systems (2.9) and (2.10), and we have seen that for $\left(g\right.$ Lip $\left.^{C}\right)$ to be fulfilled, it suffices to show a global Lipschitz property $(g L i p)$ for $f, g h$ and $k$ (note that $g$ satisfying ( $g$ Lip) also implies that the solution $y\left(\theta ; t_{*}, y_{0}\right)$ exists and is unique on the entire positive half line for every $\left.y_{0}\right)$. It is not hard to see that ( $g$ Lip) holds for $h$ and $k$, and shortly we will prove that $f$ and $g$ satisfy this property as well.

First we state a proposition about the nonnegativity of solutions for system $\left(L^{*}\right)$ by applying Corollary 2.12 from the general theory (the conditions in it clearly hold), moreover we claim without proof that nonnegative initial data give rise to nonnegative solutions in system $\left(T^{*}\right)$ (since it is a compact form of the system describing the evolution of densities during travel, which is formulated as the standard SEAIR model). The equivalences of systems $(L)$ and $\left(L^{*}\right)$, and systems $(T)$ and $\left(T^{*}\right)$ imply that the results obtained for $\left(L^{*}\right)$ and $\left(T^{*}\right)$ automatically hold for $(L)$ and $(T)$, respectively. Henceforth, we formulate our statements for systems $(L)$ and $(T)$.

Proposition 3.1. For any $\Phi \in C_{+}$, the solution of system $(L)$ is nonnegative where it exists. System (T) preserves nonnegativity for nonnegative initial values.

We arrive to the following lemma which proves that the global Lipschitz condition is satisfied for $f$ and $g$ on the nonnegative cone of $\mathbb{R}^{20}$. With the use of Proposition 3.1 and the theory in Section 2.3, this yields the existence and uniqueness of the solution of $(L)$.

Lemma 3.2. Functions $f$ and $g$, as defined for the SEAIR model, satisfy the global Lipschitz condition ( $g$ Lip) on each bounded subset of $\mathbb{R}_{+}^{20}$.

Proof. Due to the similarities in the definitions of $f$ and $g$, here we prove the condition only for one of them, e.g., for $f$. The function $f: \mathbb{R}^{20} \rightarrow \mathbb{R}^{20}$ possesses the global Lipschitz condition (gLip) if there exists a Lipschitz constant $K>0$ such that $|f(z)-f(w)|_{20} \leq$ $K|z-w|_{20}$ holds for any $z, w \in \mathbb{R}_{+}^{20}$ (in the sequel we will use $|\cdot|$ to denote any Euclidean vector norm for convenience). First we show that there exist constants $K_{1}, \ldots K_{5}>0$, such that inequalities $\left|f_{i}(z)-f_{i}(w)\right| \leq K_{i}|z-w|, i=1, \ldots, 5$, hold; then we proceed as we 
argue that the formulas of $f_{6}, \ldots, f_{10}, f_{11}, \ldots, f_{15}$ and $f_{16}, \ldots, f_{20}$ differ only in constants from $f_{1}, \ldots, f_{5}$ and henceforth $K_{6}, \ldots, K_{20}$ can be obtained similarly as $K_{1}, \ldots, K_{5}$. For $z, w \in \mathbb{R}_{+}^{20}, z \neq 0, w \neq 0$, it holds that

$$
\begin{aligned}
\left|f_{1}(z)-f_{1}(w)\right|= & \mid \Lambda_{1}-\frac{z_{1}}{\sum_{j=1}^{10} z_{j}}\left(\beta_{1}^{r r}\left(z_{4}+\rho z_{3}\right)+\beta_{1}^{v r}\left(z_{9}+\rho z_{8}\right)\right)-\left(d_{1}^{r}+\alpha_{1}\right) z_{1} \\
& -\Lambda_{1}+\frac{w_{1}}{\sum_{j=1}^{10} w_{j}}\left(\beta_{1}^{r r}\left(w_{4}+\rho w_{3}\right)+\beta_{1}^{v r}\left(w_{9}+\rho w_{8}\right)\right)+\left(d_{1}^{r}+\alpha_{1}\right) w_{1} \mid \\
\leq & \left(d_{1}^{r}+\alpha_{1}\right)\left|w_{1}-z_{1}\right|+\beta_{1}^{r r} \mid \frac{w_{1} w_{4}}{\sum_{j=1}^{10} w_{j}}+\frac{\rho w_{1} w_{3}}{\sum_{j=1}^{10} w_{j}}-\frac{z_{1} z_{4}}{\sum_{j=1}^{10} z_{j}} \\
& -\frac{\rho z_{1} z_{3}}{\sum_{j=1}^{10} z_{j}}\left|+\beta_{1}^{v r}\right| \frac{w_{1} w_{9}}{\sum_{j=1}^{10} w_{j}}+\frac{\rho w_{1} w_{8}}{\sum_{j=1}^{10} w_{j}}-\frac{z_{1} z_{9}}{\sum_{j=1}^{10} z_{j}}-\frac{\rho z_{1} z_{8}}{\sum_{j=1}^{10} z_{j}} \mid .
\end{aligned}
$$

For an expression of the form $\left|\frac{a_{1} a_{2}}{\sum_{j=1}^{10} a_{j}}-\frac{b_{1} b_{2}}{\sum_{j=1}^{10} b_{j}}\right|\left(a_{j}, b_{j} \in \mathbb{R}_{+}\right)$, the following estimation can be derived:

$$
\begin{aligned}
\left|\frac{a_{1} a_{2}}{\sum_{j=1}^{10} a_{j}}-\frac{b_{1} b_{2}}{\sum_{j=1}^{10} b_{j}}\right| \leq & \left|\frac{a_{1} a_{2}}{\sum_{j=1}^{10} a_{j}}-\frac{a_{1} b_{2}}{\sum_{j=1}^{10} a_{j}}\right|+\left|\frac{a_{1} b_{2}}{\sum_{j=1}^{10} a_{j}}-\frac{a_{1} b_{2}}{\sum_{j=1}^{10} b_{j}}\right| \\
& +\left|\frac{a_{1} b_{2}}{\sum_{j=1}^{10} b_{j}}-\frac{b_{1} b_{2}}{\sum_{j=1}^{10} b_{j}}\right| \\
= & \left|a_{2}-b_{2}\right| \frac{a_{1}}{\sum_{j=1}^{10} a_{j}}+\left|\sum_{j=1}^{10} b_{j}-\sum_{j=1}^{10} a_{j}\right| \frac{a_{1} b_{2}}{\left(\sum_{j=1}^{10} a_{j}\right)\left(\sum_{j=1}^{10} b_{j}\right)} \\
& +\left|a_{1}-b_{1}\right| \frac{b_{2}}{\sum_{j=1}^{10} b_{j}} \\
\leq & \left|a_{2}-b_{2}\right| \frac{a_{1}}{\sum_{j=1}^{10} a_{j}}+\left|a_{1}-b_{1}\right| \frac{b_{2}}{\sum_{j=1}^{10} b_{j}} \\
& +\sum_{j=1}^{10}\left|b_{j}-a_{j}\right| \frac{a_{1} b_{2}}{\left(\sum_{j=1}^{10} a_{j}\right)\left(\sum_{j=1}^{10} b_{j}\right)} \\
\leq & \left|a_{2}-b_{2}\right|+\left|a_{1}-b_{1}\right|+\sum_{j=1}^{10}\left|b_{j}-a_{j}\right|,
\end{aligned}
$$

and we use that the inequality $\left|w_{j}-z_{j}\right| \leq|w-z|$ holds for each $j \in\{1, \ldots, 10\}$ to get that

$$
\begin{aligned}
\left|f_{1}(z)-f_{1}(w)\right| \leq & \left(d_{1}^{r}+\alpha_{1}\right)\left|w_{1}-z_{1}\right|+\beta_{1}^{r r}(12|w-z|+12 \rho|w-z|) \\
& +\beta_{1}^{v r}(12|w-z|+12 \rho|w-z|) \\
\leq & \left(d_{1}^{r}+\alpha_{1}+12(1+\rho)\left(\beta_{1}^{r r}+\beta_{1}^{v r}\right)\right)|w-z| .
\end{aligned}
$$

By defining $K_{1}=d_{1}^{r}+\alpha_{1}+12(1+\rho)\left(\beta_{1}^{r r}+\beta_{1}^{v r}\right)$ we see that $\left|f_{1}(z)-f_{1}(w)\right| \leq K_{1}|w-z|$ 
holds, furthermore one can derive that

$$
\begin{aligned}
\left|f_{2}(z)-f_{2}(w)\right|= & \mid \frac{z_{1}}{\sum_{j=1}^{10} z_{j}}\left(\beta_{1}^{r r}\left(z_{4}+\rho z_{3}\right)+\beta_{1}^{v r}\left(z_{9}+\rho z_{8}\right)\right)-\left(d_{1}^{r}+\mu_{E}+\alpha_{1}\right) z_{1} \\
& -\frac{w_{1}}{\sum_{j=1}^{10} w_{j}}\left(\beta_{1}^{r r}\left(w_{4}+\rho w_{3}\right)+\beta_{1}^{v r}\left(w_{9}+\rho w_{8}\right)\right)+\left(d_{1}^{r}+\mu_{E}+\alpha_{1}\right) w_{1} \mid \\
\leq & \left(d_{1}^{r}+\mu_{E}+\alpha_{1}+12(1+\rho)\left(\beta_{1}^{r r}+\beta_{1}^{v r}\right)\right)|w-z|,
\end{aligned}
$$

where $d_{1}^{r}+\mu_{E}+\alpha_{1}+12(1+\rho)\left(\beta_{1}^{r r}+\beta_{1}^{v r}\right)$ is a suitable choice for $K_{2}$ in $\left|f_{2}(z)-f_{2}(w)\right| \leq$ $K_{2}|w-z|$. Clearly, by choosing $K_{3}=(1-p) \mu_{E}+d_{1}^{r}+\mu_{A}+\alpha_{1}$ and $K_{4}=p \mu_{E}+d_{1}^{r}+\mu_{I}+\delta+\alpha_{1}$, the inequalities

$$
\begin{aligned}
\left|f_{3}(z)-f_{3}(w)\right| & =\left|(1-p) \mu_{E} z_{2}-\left(d_{1}^{r}+\mu_{A}+\alpha_{1}\right) z_{3}-(1-p) \mu_{E} w_{2}+\left(d_{1}^{r}+\mu_{A}+\alpha_{1}\right) w_{3}\right| \\
& \leq\left((1-p) \mu_{E}+d_{1}^{r}+\mu_{A}+\alpha_{1}\right)|w-z|, \\
\left|f_{4}(z)-f_{4}(w)\right| & =\left|p \mu_{E} z_{2}-\left(d_{1}^{r}+\mu_{I}+\delta+\alpha_{1}\right) z_{4}-p \mu_{E} w_{2}+\left(d_{1}^{r}+\mu_{I}+\delta+\alpha_{1}\right) w_{4}\right| \\
& \leq\left(p \mu_{E}+d_{1}^{r}+\mu_{A}+\delta+\alpha_{1}\right)|w-z|,
\end{aligned}
$$

yield $\left|f_{3}(z)-f_{3}(w)\right| \leq K_{3}|w-z|$ and $\left|f_{4}(z)-f_{4}(w)\right| \leq K_{4}|w-z|$, and last $\left|f_{5}(z)-f_{5}(w)\right| \leq$ $K_{5}|w-z|$ arises from

$$
\begin{aligned}
\left|f_{5}(z)-f_{5}(w)\right| & =\left|\mu_{A} z_{3}+\mu_{I} z_{4}-\left(d_{1}^{r}+\alpha_{1}\right) z_{5}-\mu_{A} w_{3}-\mu_{I} w_{4}+\left(d_{1}^{r}+\alpha_{1}\right) w_{5}\right| \\
& \leq\left(\mu_{A}+\mu_{I}+d_{1}^{r}+\alpha_{1}\right)|w-z|
\end{aligned}
$$

with $K_{5}=\mu_{A}+\mu_{I}+d_{1}^{r}+\alpha_{1}$.

As pointed out earlier in the proof, the constants $K_{6}, \ldots, K_{20}$ can be derived in a similar way. To obtain $K$, the global Lipschitz constant for $f$, we simply let $K=\sqrt{\sum_{j=1}^{20}\left(K_{i}\right)^{2}}$. The proof is complete.

We conclude that all conditions for the general existence and uniqueness theorem are satisfied for the SEAIR model. As elaborated earlier, the solution exists for all positive times.

Proposition 3.3. For any fixed $t_{*}$ and initial data, there exists a unique solution of system (T) on $[0, \infty)$.

Theorem 3.4. For any initial data $\Phi \in C_{+}$, there exists a unique solution of system $(L)$ defined on $[-\tau, \infty)$.

Next we turn our attention to steady-state solutions and long-term behavior in the model. We define the disease free subspace $C_{+}^{d f}$ as

$$
C_{+}^{d f}=\left\{\Phi \mid \Phi=\left(\varphi_{S, j}^{r}, \hat{0}, \hat{0}, \hat{0}, \varphi_{R, j}^{r}, \varphi_{S, j}^{v}, \hat{0}, \hat{0}, \hat{0}, \varphi_{R, j}^{v}\right)^{T}\right\} \subset C_{+},
$$


where $\hat{0}$ denotes the constant 0 function. If $\Phi \in C_{+}^{d f}$, then

$$
E_{j}^{r}(t)=E_{j}^{v}(t)=A_{j}^{r}(t)=A_{j}^{v}(t)=I_{j}^{r}(t)=I_{j}^{v}(t) \equiv 0
$$

for all $t \geq 0$, hence the disease free subspace is positively invariant.

Proposition 3.5. In the disease free subspace $C_{+}^{d f}$ there exists a unique positive equilibrium of system $(L)$ which is globally asymptotically stable in $C_{+}^{d f}$.

Proof. Using the definitions of $N_{j}^{r}$ and $N_{j}^{v}$ in Section 3.2, for these variables we derive the following differential equation system

$$
\begin{aligned}
& \dot{N}_{j}^{r}(t)=\Lambda_{j}-\left(d_{j}^{r}+\alpha_{j}\right) N_{j}^{r}(t)+\gamma_{k} N_{k}^{v}(t-\tau), \\
& \dot{N}_{j}^{v}(t)=-\left(d_{j}^{v}+\gamma_{j}\right) N_{j}^{v}(t)+\alpha_{k} N_{k}^{r}(t-\tau),
\end{aligned}
$$

where $j, k \in\{1,2\}, j \neq k$. One can find that the positive equilibrium $\left(\hat{N}_{1}^{r}, \hat{N}_{1}^{v}, \hat{N}_{2}^{r}, \hat{N}_{2}^{v}\right)$ is given by

$$
\left(\begin{array}{c}
\hat{N}_{1}^{r} \\
\hat{N}_{1}^{v} \\
\hat{N}_{2}^{r} \\
\hat{N}_{2}^{v}
\end{array}\right)=\left(\begin{array}{cccc}
d_{1}^{r}+\alpha_{1} & 0 & 0 & -\gamma_{2} \\
0 & d_{1}^{v}+\gamma_{1} & -\alpha_{2} & 0 \\
0 & -\gamma_{2} & d_{2}^{r}+\alpha_{2} & 0 \\
-\alpha_{2} & 0 & 0 & d_{2}^{v}+\gamma_{2}
\end{array}\right)^{-1}\left(\begin{array}{c}
\Lambda_{1} \\
0 \\
\Lambda_{2} \\
0
\end{array}\right)
$$

Set $M_{j}^{r}(t):=N_{j}^{r}(t)-\hat{N}_{j}^{r}, M_{j}^{v}(t):=N_{j}^{v}(t)-\hat{N}_{j}^{v}, j \in\{1,2\}$. We obtain the decoupled linear systems

$$
\begin{aligned}
& \dot{M}_{1}^{r}(t)=-\left(d_{1}^{r}+\alpha_{1}\right) M_{1}^{r}(t)+\gamma_{2} M_{2}^{v}(t-\tau), \\
& \dot{M}_{2}^{v}(t)=-\left(d_{2}^{v}+\gamma_{2}\right) M_{2}^{v}(t)+\alpha_{1} M_{1}^{r}(t-\tau),
\end{aligned}
$$

and

$$
\begin{aligned}
& \dot{M}_{2}^{r}(t)=-\left(d_{2}^{r}+\alpha_{2}\right) M_{2}^{r}(t)+\gamma_{1} M_{1}^{v}(t-\tau), \\
& \dot{M}_{1}^{v}(t)=-\left(d_{1}^{v}+\gamma_{1}\right) M_{1}^{v}(t)+\alpha_{2} M_{2}^{r}(t-\tau) .
\end{aligned}
$$

We apply the results of Suzuki and Matsunaga ([41]) to systems (3.6) and (3.7), where criteria for the stability of the trivial solution in a class of linear differential equations has been given. Since $d_{1}^{r}, d_{1}^{v}, d_{2}^{r}, d_{2}^{v}$ and $\alpha_{1}, \alpha_{2}, \gamma_{1}, \gamma_{2}$ are positive, moreover the inequalities $\left(d_{1}^{r}+\alpha_{1}\right)\left(d_{2}^{v}+\gamma_{2}\right)>\alpha_{1} \gamma_{2}$ and $\left(d_{2}^{r}+\alpha_{2}\right)\left(d_{1}^{v}+\gamma_{1}\right)>\alpha_{2} \gamma_{1}$ are satisfied, condition (16) in [41] holds. Thus, the zero solutions of systems (3.6) and (3.7) are asymptotically stable, which implies that the positive equilibrium $\left(\hat{N}_{1}^{r}, \hat{N}_{1}^{v}, \hat{N}_{2}^{r}, \hat{N}_{2}^{v}\right)$ is asymptotically stable.

Since on the disease free subspace $\frac{\mathrm{d}}{\mathrm{d} \theta} r_{j, k}^{r}\left(\theta ; t_{*}\right)=0$ and $\frac{\mathrm{d}}{\mathrm{d} \theta} r_{j, k}^{v}\left(\theta ; t_{*}\right)=0$, from $(I V T)$ we obtain $r_{k, j}^{v}(\tau ; t-\tau)=\gamma_{k} R_{k}^{v}(t-\tau)$ and $r_{k, j}^{r}(\tau ; t-\tau)=\alpha_{k} R_{k}^{r}(t-\tau)$ for $j, k \in\{1,2\}, j \neq k$. Consider the following subsystem

$$
\begin{aligned}
\dot{R}_{j}^{r}(t) & =-\left(d_{j}^{r}+\alpha_{j}\right) R_{j}^{r}(t)+\gamma_{k} R_{k}^{v}(t-\tau), \\
\dot{R}_{j}^{v}(t) & =-\left(d_{j}^{v}+\gamma_{j}\right) R_{j}^{v}(t)+\alpha_{k} R_{k}^{r}(t-\tau) .
\end{aligned}
$$


With similar argument as for systems (3.6) and (3.7), we obtain that the equilibrium $\left(\hat{R}_{1}^{r}, \hat{R}_{1}^{v}, \hat{R}_{2}^{r}, \hat{R}_{2}^{v}\right)=(0,0,0,0)$ is asymptotically stable. We conclude that $R_{j}^{r}(t), R_{j}^{v}(t) \rightarrow 0$ as $t \rightarrow \infty, j, k \in\{1,2\}, j \neq k$. In the disease free subspace, $N_{j}^{r}(t)=S_{j}^{r}(t)+R_{j}^{r}(t)$ and $N_{j}^{v}(t)=S_{j}^{v}(t)+R_{j}^{v}(t), j \in\{1,2\}$, thus $S_{j}^{r}(t) \rightarrow \hat{N}_{j}^{r}$ and $S_{j}^{v}(t) \rightarrow \hat{N}_{j}^{v}$ as $t \rightarrow \infty$.

Henceforward, in the disease free subspace the solutions of $(L)$ converge to the equilibrium $\hat{N}=\left(\hat{N}_{1}^{r}, 0,0,0,0, \hat{N}_{1}^{v}, 0,0,0,0, \hat{N}_{2}^{r}, 0,0,0,0, \hat{N}_{2}^{v}, 0,0,0,0\right)^{T}$.

As an immediate consequence of Propositions 3.1 and 3.5, we have that in the disease free subspace the solutions of $(L)$ are bounded.

Proposition 3.6. If $\delta=0$ then the total populations $\left(N_{1}^{r}(t), N_{1}^{v}(t), N_{2}^{r}(t), N_{2}^{v}(t)\right)$ converge to $\left(\hat{N}_{1}^{r}, \hat{N}_{1}^{v}, \hat{N}_{2}^{r}, \hat{N}_{2}^{v}\right)$, which is given by (3.5).

Proof. If $\delta=0$, then it is easy to see that $N_{1}^{r}(t), N_{1}^{v}(t), N_{2}^{r}(t), N_{2}^{v}(t)$ satisfy system (3.4), hence we obtain the same positive equilibrium $\left(\hat{N}_{1}^{r}, \hat{N}_{1}^{v}, \hat{N}_{2}^{r}, \hat{N}_{2}^{v}\right)$ which is globally asymptotically stable.

Proposition 3.7. Solutions of system (L) are bounded.

Proof. For any $\Phi \in C_{+}$, the system of $N_{1}^{r}(t), N_{1}^{v}(t), N_{2}^{r}(t), N_{2}^{v}(t)$ becomes

$$
\begin{aligned}
& \dot{N}_{j}^{r}(t)=\Lambda_{j}-\left(d_{j}^{r}+\alpha_{j}\right) N_{j}^{r}(t)-\delta I_{j}^{r}(t)+\gamma_{k} N_{k}^{v}(t-\tau), \\
& \dot{N}_{j}^{v}(t)=-\left(d_{j}^{v}+\gamma_{j}\right) N_{j}^{v}(t)-\delta I_{j}^{v}(t)+\alpha_{k} N_{k}^{r}(t-\tau) .
\end{aligned}
$$

By Proposition 3.1, $N_{j}^{m}(u), I_{j}^{m}(u), j \in\{1,2\}, m \in\{v, r\}$, are nonnegative, thus by a standard comparison argument (see Theorem 4.1 in [23]), solutions of (3.8) are bounded by the solutions of (3.4), which are convergent according to Proposition 3.5. Thus, we conclude that $N_{j}^{r}(t)$ and $N_{j}^{v}(t), j \in\{1,2\}$, are bounded. Since

$$
\begin{aligned}
& 0 \leq S_{j}^{r}(t), E_{j}^{r}(t), A_{j}^{r}(t), I_{j}^{r}(t), R_{j}^{r}(t) \leq N_{j}^{r}(t), \\
& 0 \leq S_{j}^{v}(t), E_{j}^{v}(t), A_{j}^{v}(t), I_{j}^{v}(t), R_{j}^{v}(t) \leq N_{j}^{v}(t),
\end{aligned}
$$

solutions of system $(L)$ are bounded.

\subsection{The basic reproduction number}

The basic reproduction number $\left(\mathcal{R}_{0}\right)$ is a central quantity in epidemiology as it determines the average number of secondary infections caused by a typical infected individual during the period of infectiousness, who was introduced into a completely susceptible population. In Section 3.2 we introduced a dynamical model describing the temporal evolution of an infectious disease in and between two regions connected by public transportation. This section is devoted to the computation of the basic (global) reproduction number of the 
model. It is defined as the dominant eigenvalue of the next generation matrix (NGM), as introduced in $[11,12]$. First we apply some modifications on the model setup and calculate the NGM. Then we show that the reproduction number works as a threshold quantity for the stability of the disease free equilibrium of the system.

We define the local reproduction numbers as we consider our model in the absence of travel. In this case the two regions are isolated, hence to obtain the (local) reproduction number of region $j, j \in\{1,2\}$, it suffices to follow a typical infected individual during the infectious period in region $j$. Given that the probability of developing symptoms is $p$, the reduction of infectiousness of asymptomatic infecteds is $\rho$, and the average length of the infectious period in classes $I$ and $A$ is $1 / \mu_{I}$ and $1 / \mu_{A}$, respectively, we arrive to the formula

$$
\mathcal{R}_{L, j}=\beta_{j}^{r r}\left(\frac{p}{\mu_{I}}+(1-p) \frac{\rho}{\mu_{A}}\right)
$$

where $\beta_{j}^{r r}$ is the transmission rate in region $j$. In case of isolated regions, the global reproduction number arises as the maximum of the local reproduction numbers. However, the unlimited number of travels and the possibility of disease transmission during travel make it very complicated to trace secondary cases if we incorporate air transportation.

In this section, we neglect the transition from exposed to infected, and from infected to recovered classes during travel, i.e., we assume that $\mu_{E}^{T}=\mu_{A}^{T}=\mu_{I}^{T}=0$. Although with this limitation we ignore the possibility of going to the infected classes or to the recovered compartment on the plane, this assumption also ensures that individuals do not undergo multiple disease states during the same travel. For realistic values of the travel duration $\tau$ it is quite unrealistic to expect that, for instance, someone who was susceptible before travel arrives as recovered upon completing the travel. As shown below, this hypothesis also allows us to calculate the basic reproduction number explicitly, and the most important part of the transmission dynamics during travel, namely exposure of susceptibles to the infection, is still fully considered in the modified model.

With the assumption that $\mu_{E}^{T}=\mu_{A}^{T}=\mu_{I}^{T}=0$ and the notations

$$
\begin{aligned}
& F_{j, k}^{T}\left(\theta ; t_{*}\right)=\frac{\beta^{T}}{n_{j, k}\left(\theta ; t_{*}\right)}\left(i_{j, k}^{r}\left(\theta ; t_{*}\right)+i_{j, k}^{v}\left(\theta ; t_{*}\right)+\rho\left(a_{j, k}^{r}\left(\theta ; t_{*}\right)+a_{j, k}^{v}\left(\theta ; t_{*}\right)\right)\right), \\
& n_{j, k}\left(\theta ; t_{*}\right)=\alpha_{j} N_{j}^{r}\left(t_{*}\right)+\gamma_{j} N_{j}^{v}\left(t_{*}\right),
\end{aligned}
$$


our system $(T)$ becomes

$$
\left\{\begin{array}{l}
\frac{\mathrm{d}}{\mathrm{d} \theta} s_{j, k}^{r}\left(\theta ; t_{*}\right)=-s_{j, k}^{r}\left(\theta ; t_{*}\right) F_{j, k}^{T}\left(\theta ; t_{*}\right), \\
\frac{\mathrm{d}}{\mathrm{d} \theta} e_{j, k}^{r}\left(\theta ; t_{*}\right)=s_{j, k}^{r}\left(\theta ; t_{*}\right) F_{j, k}^{T}\left(\theta ; t_{*}\right), \\
\frac{\mathrm{d}}{\mathrm{d} \theta} a_{j, k}^{r}\left(\theta ; t_{*}\right)=\frac{\mathrm{d}}{\mathrm{d} \theta} i_{j, k}^{r}\left(\theta ; t_{*}\right)=\frac{\mathrm{d}}{\mathrm{d} \theta} r_{j, k}^{r}\left(\theta ; t_{*}\right)=0 \\
\frac{\mathrm{d}}{\mathrm{d} \theta} s_{j, k}^{v}\left(\theta ; t_{*}\right)=-s_{j, k}^{v}\left(\theta ; t_{*}\right) F_{j, k}^{T}\left(\theta ; t_{*}\right), \\
\frac{\mathrm{d}}{\mathrm{d} \theta} e_{j, k}^{v}\left(\theta ; t_{*}\right)=s_{j, k}^{v}\left(\theta ; t_{*}\right) F_{j, k}^{T}\left(\theta ; t_{*}\right), \\
\frac{\mathrm{d}}{\mathrm{d} \theta} a_{j, k}^{v}\left(\theta ; t_{*}\right)=\frac{\mathrm{d}}{\mathrm{d} \theta} i_{j, k}^{v}\left(\theta ; t_{*}\right)=\frac{\mathrm{d}}{\mathrm{d} \theta} r_{j, k}^{v}\left(\theta ; t_{*}\right)=0
\end{array}\right.
$$

where $j, k \in\{1,2\}, j \neq k$. Using systems $\left(T^{\prime}\right)$ and (IVT), we obtain the densities of asymptomatic, symptomatic infected and recovered individuals during travel with respect to $\theta$ as

$$
\begin{array}{ll}
a_{j, k}^{r}\left(\theta ; t_{*}\right)=a_{j, k}^{r}\left(0 ; t_{*}\right)=\alpha_{j} A_{j}^{r}\left(t_{*}\right), & a_{j, k}^{v}\left(\theta ; t_{*}\right)=a_{j, k}^{v}\left(0 ; t_{*}\right)=\gamma_{j} A_{j}^{v}\left(t_{*}\right), \\
i_{j, k}^{r}\left(\theta ; t_{*}\right)=i_{j, k}^{r}\left(0 ; t_{*}\right)=\alpha_{j} I_{j}^{r}\left(t_{*}\right), & i_{j, k}^{v}\left(\theta ; t_{*}\right)=i_{j, k}^{v}\left(0 ; t_{*}\right)=\gamma_{j} I_{j}^{v}\left(t_{*}\right), \\
r_{j, k}^{r}\left(\theta ; t_{*}\right)=r_{j, k}^{r}\left(0 ; t_{*}\right)=\alpha_{j} R_{j}^{r}\left(t_{*}\right), & r_{j, k}^{v}\left(\theta ; t_{*}\right)=r_{j, k}^{v}\left(0 ; t_{*}\right)=\gamma_{j} R_{j}^{v}\left(t_{*}\right)
\end{array}
$$

for all $t_{*}, \theta \in[0, \tau]$ and $j, k \in\{1,2\}, j \neq k$. Then, using (3.9), the force of infection $F_{j, k}^{T}$ can be obtained as

$$
F_{j, k}^{T}\left(\theta ; t_{*}\right)=\beta^{T} \frac{\alpha_{j} I_{j}^{r}\left(t_{*}\right)+\gamma_{j} I_{j}^{v}\left(t_{*}\right)+\rho\left(\alpha_{j} A_{j}^{r}\left(t_{*}\right)+\gamma_{j} A_{j}^{v}\left(t_{*}\right)\right)}{\alpha_{j} N_{j}^{r}\left(t_{*}\right)+\gamma_{j} N_{j}^{v}\left(t_{*}\right)},
$$

where $\theta \in[0, \tau]$ and $j, k \in\{1,2\}, j \neq k$, and we can determine the density of susceptible individuals during travel for $\theta \in[0, \tau], j, k \in\{1,2\}, j \neq k$ as

$$
\begin{aligned}
s_{j, k}^{r}\left(\theta ; t_{*}\right) & =s_{j, k}^{r}\left(0 ; t_{*}\right) e^{-\int_{0}^{\theta} F_{j, k}^{T}\left(\nu, t_{*}\right) d \nu} \\
& =\alpha_{j} S_{j}^{r}\left(t_{*}\right) e^{-\theta \beta^{T} \frac{\alpha_{j} I_{j}^{r}\left(t_{*}\right)+\gamma_{j} I_{j}^{v}\left(t_{*}\right)+\rho\left(\alpha_{j} A_{j}^{r}\left(t_{*}\right)+\gamma_{j} A_{j}^{v}\left(t_{*}\right)\right)}{\alpha_{j} N_{j}^{r}\left(t_{*}\right)+\gamma_{j} N_{j}^{v}\left(t_{*}\right)}}, \\
s_{j, k}^{v}\left(\theta ; t_{*}\right) & =s_{j, k}^{v}\left(0 ; t_{*}\right) e^{-\int_{0}^{\theta} F_{j, k}^{T}\left(\nu, t_{*}\right) d \nu} \\
& =\gamma_{j} S_{j}^{v}\left(t_{*}\right) e^{-\theta \beta^{T} \frac{\alpha_{j} I_{j}^{r}\left(t_{*}\right)+\gamma_{j} I_{j}^{v}\left(t_{*}\right)+\rho\left(\alpha_{j} A_{j}^{r}\left(t_{*}\right)+\gamma_{j} A_{j}^{v}\left(t_{*}\right)\right)}{\alpha_{j} N_{j}^{r}\left(t_{*}\right)+\gamma_{j} N_{j}^{v}\left(t_{*}\right)}} .
\end{aligned}
$$

Last, using the definition of $n_{j, k}^{m}\left(\theta ; t_{*}\right)(j, k \in\{1,2\}, m \in\{r, v\})$, we obtain the density of 
exposed individuals during travel as

$$
\begin{aligned}
e_{j, k}^{r}\left(\theta ; t_{*}\right) & =n_{j, k}^{r}\left(\theta ; t_{*}\right)-a_{j, k}^{r}\left(\theta ; t_{*}\right)-i_{j, k}^{r}\left(\theta ; t_{*}\right)-r_{j, k}^{r}\left(\theta ; t_{*}\right)-s_{j, k}^{r}\left(\theta ; t_{*}\right) \\
& =\alpha_{j} N_{j}^{r}\left(t_{*}\right)-\alpha_{j} A_{j}^{r}\left(t_{*}\right)-\alpha_{j} I_{j}^{r}\left(t_{*}\right)-\alpha_{j} R_{j}^{r}\left(t_{*}\right)-s_{j, k}^{r}\left(\theta ; t_{*}\right) \\
& =\alpha_{j}\left(S_{j}^{r}\left(t_{*}\right)\left(1-e^{-\theta \beta^{T} \frac{\alpha_{j} I_{j}^{r}\left(t_{*}\right)+\gamma_{j} I_{j}^{v}\left(t_{*}\right)+\rho\left(\alpha_{j} A_{j}^{r}\left(t_{*}\right)+\gamma_{j} A_{j}^{v}\left(t_{*}\right)\right)}{\alpha_{j} N_{j}^{r}\left(t_{*}\right)+\gamma_{j} N_{j}^{v}\left(t_{*}\right)}}\right)+E_{j}^{r}\left(t_{*}\right)\right), \\
e_{j, k}^{v}\left(\theta ; t_{*}\right) & =n_{j, k}^{v}\left(\theta ; t_{*}\right)-a_{j, k}^{v}\left(\theta ; t_{*}\right)-i_{j, k}^{v}\left(\theta ; t_{*}\right)-r_{j, k}^{v}\left(\theta ; t_{*}\right)-s_{j, k}^{v}\left(\theta ; t_{*}\right) \\
& =\gamma_{j} N_{j}^{v}\left(t_{*}\right)-\gamma_{j} A_{j}^{v}\left(t_{*}\right)-\gamma_{j} I_{j}^{v}\left(t_{*}\right)-\gamma_{j} R_{j}^{v}\left(t_{*}\right)-s_{j, k}^{v}\left(\theta ; t_{*}\right) \\
& =\gamma_{j}\left(S_{j}^{v}\left(t_{*}\right)+E_{j}^{v}\left(t_{*}\right)\right)-\gamma_{j} S_{j}^{v}\left(t_{*}\right) e^{-\theta \beta^{T} \frac{\alpha_{j} I_{j}^{r}\left(t_{*}\right)+\gamma_{j} I_{j}^{v}\left(t_{*}\right)+\rho\left(\alpha_{j} A_{j}^{r}\left(t_{*}\right)+\gamma_{j} A_{j}^{v}\left(t_{*}\right)\right)}{\alpha_{j} N_{j}^{r}\left(t_{*}\right)+\gamma_{j} N_{j}^{v}\left(t_{*}\right)}} .
\end{aligned}
$$

Choosing $\theta=\tau$ and $t_{*}=t-\tau$, the inflow terms $s_{j, k}^{m}(\tau ; t-\tau), e_{j, k}^{m}(\tau ; t-\tau), a_{j, k}^{m}(\tau ; t-\tau)$, $i_{j, k}^{m}(\tau ; t-\tau)$ and $r_{j, k}^{m}(\tau ; t-\tau)(j, k \in\{1,2\}, m \in\{r, v\})$, determined in (3.9), (3.10) and (3.11), arise as delay terms of $S_{j}^{m}, E_{j}^{m}, A_{j}^{m}, I_{j}^{m}$ and $R_{j}^{m}$.

Notation 3.8. In the sequel, we denote by $\left(L^{\prime}\right)$ the special case of $(L)$ with the particular inflow terms defined in (3.9), (3.10) and (3.11).

We use the notations of Section 3.3 and define $\bar{W}: \mathbb{R}^{20} \rightarrow \mathbb{R}^{20}$ as $\bar{W}_{i}$ equals the inflow term of the right-hand side of the equation for $x_{i}$ in $\left(L^{\prime}\right)$. For instance,

$$
\bar{W}_{1}(v)=\gamma_{2} v_{16} e^{-\tau \beta^{T} \frac{\alpha_{2} v_{14}+\gamma_{2} v_{19}+\rho\left(\alpha_{2} v_{13}+\gamma_{2} v_{18}\right)}{\alpha_{2} \sum_{j=11}^{15} v_{j}+\gamma_{2} \sum_{j=16}^{20} v_{j}}}, \bar{W}_{18}(v)=\alpha_{1} v_{3} .
$$

System $\left(L^{\prime}\right)$ can be written in the compact form of

$$
x^{\prime}(t)=\overline{\mathcal{F}}(x(t), x(t-\tau)),
$$

where $\overline{\mathcal{F}}(x(t), x(t-\tau))=f(x(t))+\bar{W}(x(t-\tau)), \overline{\mathcal{F}}: \mathbb{R}^{20} \times \mathbb{R}^{20} \rightarrow \mathbb{R}^{20}$. Now we focus on system $(\bar{L})$ and we detail the computation of the reproduction number.

Notice that the disease free equilibrium $\hat{N}=\left(\hat{N}_{1}^{r}, 0,0,0,0, \hat{N}_{1}^{v}, 0,0,0,0, \hat{N}_{2}^{r}, 0,0,0,0\right.$, $\left.\hat{N}_{2}^{v}, 0,0,0,0\right)$ of system $(L)$, defined in Section 3.3 , is the unique positive equilibrium of $(\bar{L})$ in the disease free subspace. In the initial stage of the epidemic, we can assume that system $(\bar{L})$ is near the equilibrium $\hat{N}$ and approximate the equations of classes $E_{j}^{m}, A_{j}^{m}, I_{j}^{m}, j \in$ $\{1,2\}, m \in\{r, v\}$, with the linear system

$$
z^{\prime}(t)=\mathcal{A} z(t)+\mathcal{B} z(t-\tau)
$$

where $z: \mathbb{R} \rightarrow \mathbb{R}^{12}, \mathcal{A}, \mathcal{B} \in \mathbb{R}^{12 \times 12}$ and $\mathcal{A}=D f(\hat{N}), \mathcal{B}=D \bar{W}(\hat{N})$ hold. The matrices $\mathcal{A}$ and $\mathcal{B}$ have the form

$$
\mathcal{A}=\left(\begin{array}{cccc}
A_{11} & A_{12} & A_{13} & A_{14} \\
A_{21} & A_{22} & A_{23} & A_{24} \\
A_{31} & A_{32} & A_{33} & A_{34} \\
A_{41} & A_{42} & A_{43} & A_{44}
\end{array}\right), \quad \mathcal{B}=\left(\begin{array}{llll}
B_{11} & B_{12} & B_{13} & B_{14} \\
B_{21} & B_{22} & B_{23} & B_{24} \\
B_{31} & B_{32} & B_{33} & B_{34} \\
B_{41} & B_{42} & B_{43} & B_{44}
\end{array}\right)
$$


where $A_{j, k}, B_{j, k} \in \mathbb{R}^{3 \times 3}$ for $j, k \in\{1,2,3,4\}$, and $A_{j, k}$ and $B_{j, k}$ can be obtained as follows:

$$
\begin{gathered}
A_{11}=\left(\begin{array}{ccc}
-\left(\mu_{E}+\alpha_{1}+d_{1}^{r}\right) & \rho \beta_{1}^{r r} \frac{\hat{N}_{1}^{r}}{\hat{N}_{1}^{r}+\hat{N}_{1}^{v}} & \beta_{1}^{r r} \frac{\hat{N}_{1}^{r}}{\hat{N}_{1}^{r}+\hat{N}_{1}^{v}} \\
(1-p) \mu_{E} & -\left(\mu_{A}+\alpha_{1}+d_{1}^{r}\right) & 0 \\
p \mu_{E} & 0 & -\left(\mu_{I}+\alpha_{1}+\delta+d_{1}^{r}\right)
\end{array}\right), \\
A_{12}=\left(\begin{array}{ccc}
0 & \rho \beta_{1}^{v r} \frac{\hat{N}_{1}^{r}}{\hat{N}_{1}^{r}+\hat{N}_{1}^{v}} & \beta_{1}^{v r} \frac{\hat{N}_{1}^{r}}{\hat{N}_{1}^{r}+\hat{N}_{1}^{v}} \\
0 & 0 & 0 \\
0 & 0 & 0
\end{array}\right),
\end{gathered}
$$

and $A_{1,3}=A_{1,4}=\mathcal{O}$, where we denote the matrix with 0 -entries by $\mathcal{O}$, moreover

$$
B_{13}=\left(\begin{array}{ccc}
0 & \frac{\tau \beta^{T} \rho \alpha_{2} \gamma_{2} \hat{N}_{2}^{v}}{\alpha_{2} \hat{N}_{2}^{r}+\gamma_{2} \hat{N}_{2}^{v}} & \frac{\tau \beta^{T} \alpha_{2} \gamma_{2} \hat{N}_{2}^{v}}{\alpha_{2} \hat{N}_{2}^{r}+\gamma_{2} \hat{N}_{2}^{v}} \\
0 & 0 & 0 \\
0 & 0 & 0
\end{array}\right), \quad B_{14}=\left(\begin{array}{ccc}
\gamma_{2} & \frac{\tau \beta^{T} \rho \gamma_{2}^{2} \hat{N}_{2}^{v}}{\alpha_{2} \hat{N}_{2}^{r}+\gamma_{2} \hat{N}_{2}^{v}} & \frac{\tau \beta^{T} \gamma_{2}^{2} \hat{N}_{2}^{v}}{\alpha_{2} \hat{N}_{2}^{r}+\gamma_{2} \hat{N}_{2}^{v}} \\
0 & \gamma_{2} & 0 \\
0 & 0 & \gamma_{2}
\end{array}\right),
$$

and $B_{1,1}=B_{1,2}=\mathcal{O}$. The matrix elements $A_{j, k}, B_{j, k}, j \in\{2,3,4\}, k \in\{1,2,3,4\}$, can be derived similarly.

Next we decompose the matrix $\mathcal{A}+\mathcal{B}$ as $\mathbb{F}-\mathbb{V}$, where $\mathbb{F}$ is the transmission part, describing the production of new infections, and $-\mathbb{V}$ is the transition part, describing all other changes in state (see $[11,47]$ for more details and some motivation for the decomposition). We first determine what we call reproduction here, i.e., in what kind of situations do new infections occur. We define two possible ways of reproduction:

(i) a susceptible moves to exposed class while being in a region;

(ii) an exposed individual, who was susceptible before travel, arrives to a region upon completing a trip.

With this definition in mind, we obtain $\mathbb{F} \in \mathbb{R}^{12 \times 12}$ and $\mathbb{V} \in \mathbb{R}^{12 \times 12}$ as

$$
\mathbb{F}=\left(\begin{array}{llll}
F_{11} & F_{12} & F_{13} & F_{14} \\
F_{21} & F_{22} & F_{23} & F_{24} \\
F_{31} & F_{32} & F_{33} & F_{34} \\
F_{41} & F_{42} & F_{43} & F_{44}
\end{array}\right), \quad \mathbb{V}=\left(\begin{array}{llll}
V_{11} & V_{12} & V_{13} & V_{14} \\
V_{21} & V_{22} & V_{23} & V_{24} \\
V_{31} & V_{32} & V_{33} & V_{34} \\
V_{41} & V_{42} & V_{43} & V_{44}
\end{array}\right),
$$

where $F_{j, k}, V_{j, k} \in \mathbb{R}^{3 \times 3}(j, k \in\{1,2,3,4\})$. It is easy to see that

$$
F_{11}=\left(\begin{array}{ccc}
0 & \rho \beta_{1}^{r r} \frac{\hat{N}_{1}^{r}}{\hat{N}_{1}^{r}+\hat{N}_{1}^{v}} & \beta_{1}^{r r} \frac{\hat{N}_{1}^{r}}{\hat{N}_{1}^{r}+\hat{N}_{1}^{v}} \\
0 & 0 & 0 \\
0 & 0 & 0
\end{array}\right), \quad F_{12}=\left(\begin{array}{ccc}
0 & \rho \beta_{1}^{v r} \frac{\hat{N}_{1}^{r}}{\hat{N}_{1}^{r}+\hat{N}_{1}^{v}} & \beta_{1}^{v r} \frac{\hat{N}_{1}^{r}}{\hat{N}_{1}^{r}+\hat{N}_{1}^{v}} \\
0 & 0 & 0 \\
0 & 0 & 0
\end{array}\right) \text {, }
$$




$$
F_{13}=\left(\begin{array}{ccc}
0 & \frac{\tau \beta^{T} \rho \alpha_{2} \gamma_{2} \hat{N}_{2}^{v}}{\alpha_{2} \hat{N}_{2}^{r}+\gamma_{2} \hat{N}_{2}^{v}} & \frac{\tau \beta^{T} \alpha_{2} \gamma_{2} \hat{N}_{2}^{v}}{\alpha_{2} \hat{N}_{2}^{r}+\gamma_{2} \hat{N}_{2}^{v}} \\
0 & 0 & 0 \\
0 & 0 & 0
\end{array}\right), \quad F_{14}=\left(\begin{array}{ccc}
0 & \frac{\tau \beta^{T} \rho \gamma_{2}^{2} \hat{N}_{2}^{v}}{\alpha_{2} \hat{N}_{2}^{r}+\gamma_{2} \hat{N}_{2}^{v}} & \frac{\tau \beta^{T} \gamma_{2}^{2} \hat{N}_{2}^{v}}{\alpha_{2} \hat{N}_{2}^{r}+\gamma_{2} \hat{N}_{2}^{v}} \\
0 & 0 & 0 \\
0 & 0 & 0
\end{array}\right)
$$

and the elements $F_{j, k}, j \in\{2,3,4\}, k \in\{1,2,3,4\}$ arise similarly. The elements of $-\mathbb{V}$ represent rates at which individuals progress from one class to another:

$$
\begin{gathered}
V_{11}=\left(\begin{array}{ccc}
\mu_{E}+\alpha_{1}+d_{1}^{r} & 0 & 0 \\
-(1-p) \mu_{E} & \mu_{A}+\alpha_{1}+d_{1}^{r} & 0 \\
-p \mu_{E} & 0 & \mu_{I}+\alpha_{1}+\delta+d_{1}^{r}
\end{array}\right), \\
V_{14}=\left(\begin{array}{ccc}
-\gamma_{2} & 0 & 0 \\
0 & -\gamma_{2} & 0 \\
0 & 0 & -\gamma_{2}
\end{array}\right)
\end{gathered}
$$

while $V_{1,2}=V_{1,3}=\mathcal{O}$, and the elements $V_{j, k}, j \in\{2,3,4\}, k \in\{1,2,3,4\}$, can be obtained similarly. Clearly $\mathbb{F}$ is a positive matrix, that is, all of its entries are non-negative, and it is easy to check that $-\mathbb{V}$ is positive-off-diagonal, that is, all entries are non-negative except possibly those on the diagonal. For a square matrix $M$ we define the spectral bound $s(M)$ and the spectral radius $\rho(M)$ by $s(M):=\sup \{\operatorname{Re}(\lambda): \lambda \in \sigma(M)\}, \rho(M):=\sup \{|\lambda|: \lambda \in$ $\sigma(M)\}$, where $\sigma(M)$ denotes the set of eigenvalues of $M$. One can show that $s(-\mathbb{V})<0$, this is equivalent to the statement that $\mathbb{V}$ is invertible and $\mathbb{V}^{-1}$ is a positive matrix (for the proof of the equivalence, see, e.g., Lemma 6.12 in [11]). We state the following proposition.

Proposition 3.9. The zero solution of the linear delay differential equation

$$
z^{\prime}(t)=\mathcal{A} z(t)+\mathcal{B} z(t-\tau)
$$

is asymptotically stable if $\rho\left(\mathbb{F} \mathbb{V}^{-1}\right)<1$ and unstable if $\rho\left(\mathbb{F} \mathbb{V}^{-1}\right)>1$.

Proof. The principal result of Section 5, Chapter 5 in [39] is that the stability of an equilibrium of a cooperative and irreducible system of delay differential equations is the same as for an associated system of cooperative ordinary differential equations. System (3.12) is cooperative since $\mathcal{A}$ is positive-off-diagonal and $\mathcal{B}$ is a positive matrix. Every column of $\mathcal{B}$ contains at least one non-zero element, which together with the irreducibility of matrix $\mathcal{A}+\mathcal{B}$ implies that system (3.12) is irreducible. Corollary 5.2 in [39] states that the zero solution of the linear delay differential equation (3.12) is asymptotically stable (unstable) if and only if the zero solution of the linear ordinary differential equation

$$
w^{\prime}(t)=(\mathcal{A}+\mathcal{B}) w(t)
$$

is asymptotically stable (unstable). We can reformulate (3.13) as

$$
w^{\prime}(t)=(\mathbb{F}-\mathbb{V}) w(t)
$$


We have seen that $\mathbb{F}$ is a positive matrix and $-\mathbb{V}$ is a positive-off-diagonal matrix with $s(-\mathbb{V})<0$. The stability of the zero steady state of $w^{\prime}(t)=(\mathbb{F}-\mathbb{V}) w(t)$ is determined by the sign of $s(\mathbb{F}-\mathbb{V})$, which coincides with the sign of $\rho\left(\mathbb{F} \mathbb{V}^{-1}\right)-1$ (see Theorem A.1 in [13]). The proof is complete.

The statement of Proposition 3.9 extends to the nonlinear system $(\bar{L})$ by the principle of linearized stability.

Proposition 3.10. The disease free equilibrium of system $(\bar{L})$ is asymptotically stable if $\rho\left(\mathbb{F} \mathbb{V}^{-1}\right)<1$ and unstable if $\rho\left(\mathbb{F} \mathbb{V}^{-1}\right)>1$.

After obtaining stability results for $(\bar{L})$, a system of delay differential equations, we consider the following associated system of ordinary differential equations

$$
x^{\prime}(t)=f(x(t))+\bar{W}(x(t))
$$

where $\bar{W}$ was introduced previously in this section and for the definition of $x$ and $f$, see Section 3.3. The concept of the next generation matrix (NGM) of an epidemic model was introduced in Diekmann et al. [12] (and elaborated in Chapter 5 [11]) as a matrix whose elements give the number of newly infected individuals in specific categories. To obtain this matrix, one considers the equations of the system that describe the production of new infections and changes in state among infected individuals; in case of system (3.14), this infected subsystem consists of the equations for $E_{j}^{m}, A_{j}^{m}, I_{j}^{m}, j \in\{1,2\}, m \in\{r, v\}$. Clearly $\hat{N}$ works as the unique disease free equilibrium of (3.14), so we can linearize the infected subsystem about the infection-free steady state and get

$$
w^{\prime}(t)=(\mathcal{A}+\mathcal{B}) w(t)=(\mathbb{F}-\mathbb{V}) w(t)
$$

Diekmann et al. [13] refers to $\mathbb{F} \mathbb{V}^{-1} \in \mathbb{R}^{12 \times 12}$ as $\mathcal{K}_{L}$, the next generation matrix for system (3.14) with large domain. However, this matrix does not equal the next generation matrix $\mathcal{K}$ of the ODE system (3.14), because the decomposition of $\mathcal{A}+\mathcal{B}$ relates to the expected offspring of individuals of any state and not just epidemiological newborns (new infections). Since in the case of system (3.14), only states $E_{1}^{r}, E_{1}^{v}, E_{2}^{r}$ and $E_{2}^{v}$ are involved in the action of the next generation matrix $\mathcal{K}$, it is clear that $\mathcal{K} \in \mathbb{R}^{4 \times 4}$. The work [13] claims that $\rho\left(\mathcal{K}_{L}\right)=\rho(\mathcal{K})$ and it can be shown that the next generation matrix for (3.14) can be obtained as $\mathcal{K}=\left(\mathcal{K}_{L}\right)_{1,4,7,10}^{1,4,7,10}$.

Next we determine the next generation matrix $\mathcal{N}$ and the reproduction number $\mathcal{R}_{0}$ for the delay system $(\bar{L})$, then we will show that $\mathcal{N}=\mathcal{K}$, i.e., the next generation matrix for the delay system $(\bar{L})$ equals the next generation matrix for the ODE system (3.14). This result implies the following conclusion. 
Proposition 3.11. The disease free equilibrium of system $(\bar{L})$ is asymptotically stable if $\mathcal{R}_{0}<1$ and unstable if $\mathcal{R}_{0}>1$.

Proof. Since $\mathcal{R}_{0}$ is defined as the dominant eigenvalue of $\mathcal{N}$ (the existence of the dominant eigenvalue is guaranteed by the Frobenius-Perron theorem), moreover $\mathcal{K}_{L}=\mathbb{F} \mathbb{V}^{-1}$ and $\rho\left(\mathcal{K}_{L}\right)=\rho(\mathcal{K})$ hold, we obtain that $\mathcal{R}_{0}=\rho\left(\mathbb{F V}^{-1}\right)$. Then it follows from Proposition 3.10 that $\mathcal{R}_{0}$ works as a threshold quantity for the stability of the disease free equilibrium of system $(\bar{L})$.

\subsubsection{The next generation matrix}

We construct the next generation matrix $\mathcal{N}$ for system $(\bar{L})$ as we divide all exposed individuals into four groups: residents of region $1\left(E_{1}^{r}\right)$, visitors of region $1\left(E_{1}^{v}\right)$, residents of region $2\left(E_{2}^{r}\right)$ and visitors of region $2\left(E_{2}^{v}\right)$. We denote the number of new infections among individuals of region $k$ with residential status $n$ generated by an exposed individual of region $j$ with residential status $m$ by $R_{j, k}^{m, n}$, where $j, k \in\{1,2\}, m, n \in\{r, v\}$. Then $\mathcal{N} \in \mathbb{R}^{4 \times 4}$ has the form

$$
\mathcal{N}=\left(\begin{array}{llll}
R_{11}^{r r} & R_{11}^{v r} & R_{21}^{r r} & R_{21}^{v r} \\
R_{11}^{r v} & R_{11}^{v v} & R_{21}^{r v} & R_{21}^{v v} \\
R_{12}^{r r} & R_{12}^{v r} & R_{22}^{r r} & R_{22}^{v r} \\
R_{12}^{r v} & R_{12}^{v v} & R_{22}^{r v} & R_{22}^{v v}
\end{array}\right)
$$

We can obtain the elements of $\mathcal{N}$ by biological reasoning, i.e., by following a typical infected individual during the infectious period, and using our definition of reproduction. Here we detail the calculation of two elements of $\mathcal{N}$ and then show that they equal the corresponding elements of $\mathcal{K}$, the next generation matrix of the associated system of ODEs (3.14). Formulas for other elements - and hence the equalities $(\mathcal{N})_{j, k}=(\mathcal{K})_{j, k}, j, k \in\{1,2,3,4\}-$ can be derived similarly.

First, let us consider the element $R_{11}^{r r}$, namely the number of new infections in $E_{1}^{r}$ generated by an exposed resident of region 1 (a member of $E_{1}^{r}$ ). Since in the model setup we addressed no restrictions on the number of travels an individual can start, we distinguish two scenarios:

(A) After completing some even number of trips, the exposed individual turns infected (member of class $I$ or $A$ ) in region 1.

(B) After completing some odd number of trips, the exposed individual turns infected in region 2 . 
We calculate the probabilities of these events. First, the probability of turning infected after $(2 n)$ trips, $n=0,1,2, \ldots$, is obtained as

$$
\left(\frac{\alpha_{1}}{\alpha_{1}+\mu_{E}+d_{1}^{r}} \frac{\gamma_{2}}{\gamma_{2}+\mu_{E}+d_{2}^{v}}\right)^{n} \frac{\mu_{E}}{\alpha_{1}+\mu_{E}+d_{1}^{r}},
$$

which implies that

$$
\begin{aligned}
P(A) & =\sum_{n=0}^{\infty}\left(\frac{\alpha_{1}}{\alpha_{1}+\mu_{E}+d_{1}^{r}} \frac{\gamma_{2}}{\gamma_{2}+\mu_{E}+d_{2}^{v}}\right)^{n} \frac{\mu_{E}}{\alpha_{1}+\mu_{E}+d_{1}^{r}} \\
& =\frac{\mu_{E}}{\alpha_{1}+\mu_{E}+d_{1}^{r}} \sum_{n=0}^{\infty}\left(\frac{\alpha_{1}}{\alpha_{1}+\mu_{E}+d_{1}^{r}} \frac{\gamma_{2}}{\gamma_{2}+\mu_{E}+d_{2}^{v}}\right)^{n} \\
& =\frac{\mu_{E}}{\alpha_{1}+\mu_{E}+d_{1}^{r}} \cdot \frac{1}{1-\frac{\alpha_{1}}{\alpha_{1}+\mu_{E}+d_{1}^{r}} \frac{\gamma_{2}}{\gamma_{2}+\mu_{E}+d_{2}^{v}}} \\
& =\frac{\mu_{E}}{\alpha_{1}+\mu_{E}+d_{1}^{r}} \cdot \frac{\left(\alpha_{1}+\mu_{E}+d_{1}^{r}\right)\left(\gamma_{2}+\mu_{E}+d_{2}^{v}\right)}{\left(\alpha_{1}+\mu_{E}+d_{1}^{r}\right)\left(\gamma_{2}+\mu_{E}+d_{2}^{v}\right)-\alpha_{1} \gamma_{2}} \\
& =\frac{\mu_{E}\left(\gamma_{2}+\mu_{E}+d_{2}^{v}\right)}{\left(\alpha_{1}+\mu_{E}+d_{1}^{r}\right)\left(\gamma_{2}+\mu_{E}+d_{2}^{v}\right)-\alpha_{1} \gamma_{2}} .
\end{aligned}
$$

On the other hand, the probability of becoming infected after $(2 n+1)$ trips, $n=0,1,2, \ldots$, is

$$
\left(\frac{\alpha_{1}}{\alpha_{1}+\mu_{E}+d_{1}^{r}}\right)^{n+1}\left(\frac{\gamma_{2}}{\gamma_{2}+\mu_{E}+d_{2}^{v}}\right)^{n} \frac{\mu_{E}}{\mu_{E}+\gamma_{2}+d_{2}^{v}},
$$

hence we get the probability of case (B) by the calculations

$$
\begin{aligned}
P(B) & =\sum_{n=0}^{\infty} \frac{\mu_{E}}{\mu_{E}+\gamma_{2}+d_{2}^{v}} \frac{\alpha_{1}}{\alpha_{1}+\mu_{E}+d_{1}^{r}} \cdot\left(\frac{\alpha_{1}}{\alpha_{1}+\mu_{E}+d_{1}^{r}} \frac{\gamma_{2}}{\gamma_{2}+\mu_{E}+d_{2}^{v}}\right)^{n} \\
& =\frac{\mu_{E}}{\mu_{E}+\gamma_{2}+d_{2}^{v}} \cdot \frac{\alpha_{1}}{\alpha_{1}+\mu_{E}+d_{1}^{r}} \sum_{n=0}^{\infty}\left(\frac{\alpha_{1}}{\alpha_{1}+\mu_{E}+d_{1}^{r}} \frac{\gamma_{2}}{\gamma_{2}+\mu_{E}+d_{2}^{v}}\right)^{n} \\
& =\frac{\mu_{E}}{\mu_{E}+\gamma_{2}+d_{2}^{v}} \cdot \frac{\alpha_{1}}{\alpha_{1}+\mu_{E}+d_{1}^{r}} \cdot \frac{1}{1-\frac{\alpha_{1}}{\alpha_{1}+\mu_{E}+d_{1}^{r}} \frac{\gamma_{2}}{\gamma_{2}+\mu_{E}+d_{2}^{v}}} \\
& =\frac{\mu_{E}}{\mu_{E}+\gamma_{2}+d_{2}^{v}} \cdot \frac{\alpha_{1}}{\alpha_{1}+\mu_{E}+d_{1}^{r}} \cdot \frac{\left(\alpha_{1}+\mu_{E}+d_{1}^{r}\right)\left(\gamma_{2}+\mu_{E}+d_{2}^{v}\right)}{\left(\alpha_{1}+\mu_{E}+d_{1}^{r}\right)\left(\gamma_{2}+\mu_{E}+d_{2}^{v}\right)-\alpha_{1} \gamma_{2}} \\
& =\frac{\mu_{E} \alpha_{1}}{\left(\alpha_{1}+\mu_{E}+d_{1}^{r}\right)\left(\gamma_{2}+\mu_{E}+d_{2}^{v}\right)-\alpha_{1} \gamma_{2}} .
\end{aligned}
$$

In case $(\mathrm{A})$, an infected individual can transmit the disease in two ways:

(a1) as a member of class $I_{1}^{r}\left(A_{1}^{r}\right)$, i.e., the individual infects in region 1 after an even number of completed trips (counted since the individual became infected), or

(a2) after an odd number of completed trips (counted since the individual became infected), the individual is a member of class $I_{2}^{v}\left(A_{2}^{v}\right)$; the individual leaves region 2 and infects during travel from region 2 to region 1. 
Similarly, in case (B), the two ways of disease transmission are as follows:

(b1) as a member of class $I_{1}^{r}\left(A_{1}^{r}\right)$, i.e., the individual infects in region 1 after an odd number of completed trips (counted since the individual became infected), or

(b2) after an even number of completed trips (counted since the individual became infected), the individual is a member of class $I_{2}^{v}\left(A_{2}^{v}\right)$; the individual leaves region 2 and infects during travel from region 2 to region 1.

In case (a1), the expected duration of infection of an individual in class $I_{1}^{r}$ after the $(2 n)$ th trip $(n=0,1, \ldots)$ is

$$
\left(\frac{\alpha_{1}}{\alpha_{1}+\mu_{I}+\delta+d_{1}^{r}} \frac{\gamma_{2}}{\gamma_{2}+\mu_{I}+\delta+d_{2}^{v}}\right)^{n} \frac{1}{\alpha_{1}+\mu_{I}+\delta+d_{1}^{r}}
$$

we derive the total expected infection time in region 1 as

$$
\begin{aligned}
\sum_{n=0}^{\infty} & \left(\frac{\alpha_{1}}{\alpha_{1}+\mu_{I}+\delta+d_{1}^{r}} \frac{\gamma_{2}}{\gamma_{2}+\mu_{I}+\delta+d_{2}^{v}}\right)^{n} \frac{1}{\alpha_{1}+\mu_{I}+\delta+d_{1}^{r}} \\
& =\frac{1}{\alpha_{1}+\mu_{I}+\delta+d_{1}^{r}} \sum_{n=0}^{\infty}\left(\frac{\alpha_{1}}{\alpha_{1}+\mu_{I}+\delta+d_{1}^{r}} \frac{\gamma_{2}}{\gamma_{2}+\mu_{I}+\delta+d_{2}^{v}}\right)^{n} \\
& =\frac{1}{\alpha_{1}+\mu_{I}+\delta+d_{1}^{r}} \cdot \frac{1}{1-\frac{\alpha_{1}}{\alpha_{1}+\mu_{I}+\delta+d_{1}^{r}} \frac{\gamma_{2}}{\gamma_{2}+\mu_{I}+\delta+d_{2}^{v}}} \\
& =\frac{1}{\alpha_{1}+\mu_{I}+\delta+d_{1}^{r}} \cdot \frac{\left(\alpha_{1}+\mu_{I}+\delta+d_{1}^{r}\right)\left(\gamma_{2}+\mu_{I}+\delta+d_{1}^{r}\right)}{\left(\alpha_{1}+\mu_{I}+\delta+d_{1}^{r}\right)\left(\gamma_{2}+\mu_{I}+\delta+d_{2}^{v}\right)-\alpha_{1} \gamma_{2}} \\
& =\frac{\gamma_{2}+\mu_{I}+\delta+d_{2}^{v}}{\left(\alpha_{1}+\mu_{I}+\delta+d_{1}^{r}\right)\left(\gamma_{2}+\mu_{I}+\delta+d_{2}^{v}\right)-\alpha_{1} \gamma_{2}} .
\end{aligned}
$$

Similar formula holds for individuals in class $A_{1}^{r}$, so we obtain the number of new infections in case (a1) as

$$
\begin{aligned}
p & \left(\frac{\mu_{E}\left(\gamma_{2}+\mu_{E}+d_{2}^{v}\right)}{\left(\alpha_{1}+\mu_{E}+d_{1}^{r}\right)\left(\gamma_{2}+\mu_{E}+d_{2}^{v}\right)-\alpha_{1} \gamma_{2}}\right. \\
& \left.\cdot \frac{\gamma_{2}+\mu_{I}+\delta+d_{2}^{v}}{\left(\alpha_{1}+\mu_{I}+\delta+d_{1}^{r}\right)\left(\gamma_{2}+\mu_{I}+\delta+d_{2}^{v}\right)-\alpha_{1} \gamma_{2}} \beta_{1}^{r r} \frac{\hat{N}_{1}^{r}}{\hat{N}_{1}^{r}+\hat{N}_{1}^{v}}\right) \\
+(1-p) & \left(\frac{\mu_{E}\left(\gamma_{2}+\mu_{E}+d_{2}^{v}\right)}{\left(\alpha_{1}+\mu_{E}+d_{1}^{r}\right)\left(\gamma_{2}+\mu_{E}+d_{2}^{v}\right)-\alpha_{1} \gamma_{2}}\right. \\
& \left.\cdot \frac{\gamma_{2}+\mu_{A}+d_{2}^{v}}{\left(\alpha_{1}+\mu_{A}+d_{1}^{r}\right)\left(\gamma_{2}+\mu_{A}+d_{2}^{v}\right)-\alpha_{1} \gamma_{2}} \rho \beta_{1}^{r r} \frac{\hat{N}_{1}^{r}}{\hat{N}_{1}^{r}+\hat{N}_{1}^{v}}\right) .
\end{aligned}
$$

We derive the number of new infections in case (a2) similarly. Since the probability of being (symptomatic) infected just before the $(2 n+2)$ th trip $(n=0,1, \ldots)$ is

$$
\frac{\alpha_{1}}{\alpha_{1}+\mu_{I}+\delta+d_{1}^{r}}\left(\frac{\alpha_{1}}{\alpha_{1}+\mu_{I}+\delta+d_{1}^{r}} \frac{\gamma_{2}}{\gamma_{2}+\mu_{I}+\delta+d_{2}^{v}}\right)^{n} \frac{\gamma_{2}}{\gamma_{2}+\mu_{I}+\delta+d_{2}^{v}}
$$


and the duration of infection during the $(2 n+2)$ th trip is $\tau$, we get the total expected infection time of an individual of $I_{1}^{v}$ as

$$
\begin{aligned}
\sum_{n=0}^{\infty} \tau & \left(\frac{\alpha_{1}}{\alpha_{1}+\mu_{I}+\delta+d_{1}^{r}} \frac{\gamma_{2}}{\gamma_{2}+\mu_{I}+\delta+d_{2}^{v}}\right)^{n+1} \\
& =\tau \frac{\alpha_{1}}{\alpha_{1}+\mu_{I}+\delta+d_{1}^{r}} \cdot \frac{\gamma_{2}}{\gamma_{2}+\mu_{I}+\delta+d_{2}^{v}} \cdot \frac{1}{1-\frac{\alpha_{1}}{\alpha_{1}+\mu_{I}+\delta+d_{1}^{r}} \frac{\gamma_{2}}{\gamma_{2}+\mu_{I}+\delta+d_{2}^{v}}} \\
& =\tau \frac{\alpha_{1}}{\alpha_{1}+\mu_{I}+\delta+d_{1}^{r}} \cdot \frac{\gamma_{2}}{\gamma_{2}+\mu_{I}+\delta+d_{2}^{v}} \cdot \frac{\left(\alpha_{1}+\mu_{I}+\delta+d_{1}^{r}\right)\left(\gamma_{2}+\mu_{I}+\delta+d_{2}^{v}\right)}{\left(\alpha_{1}+\mu_{I}+\delta+d_{1}^{r}\right)\left(\gamma_{2}+\mu_{I}+\delta+d_{2}^{v}\right)-\alpha_{1} \gamma_{2}} \\
& =\frac{\alpha_{1}}{\left(\alpha_{1}+\mu_{I}+\delta+d_{1}^{r}\right)\left(\gamma_{2}+\mu_{I}+\delta+d_{2}^{v}\right)-\alpha_{1} \gamma_{2}} .
\end{aligned}
$$

This implies that the number of new infections in case (a2) is

$$
\begin{aligned}
p & \left(\frac{\mu_{E}\left(\gamma_{2}+\mu_{E}+d_{2}^{v}\right)}{\left(\alpha_{1}+\mu_{E}+d_{1}^{r}\right)\left(\gamma_{2}+\mu_{E}+d_{2}^{v}\right)-\alpha_{1} \gamma_{2}}\right. \\
& \left.\cdot \frac{\alpha_{1} \gamma_{2}}{\left(\alpha_{1}+\mu_{I}+\delta+d_{1}^{r}\right)\left(\gamma_{2}+\mu_{I}+\delta+d_{2}^{v}\right)-\alpha_{1} \gamma_{2}} \tau \beta^{T} \frac{\gamma_{2} \hat{N}_{2}^{v}}{\gamma_{2} \hat{N}_{2}^{v}+\alpha_{2} \hat{N}_{2}^{r}}\right) \\
+(1-p) & \left(\frac{\mu_{E}\left(\gamma_{2}+\mu_{E}+d_{2}^{v}\right)}{\left(\alpha_{1}+\mu_{E}+d_{1}^{r}\right)\left(\gamma_{2}+\mu_{E}+d_{2}^{v}\right)-\alpha_{1} \gamma_{2}}\right. \\
& \left.\cdot \frac{\alpha_{1} \gamma_{2}}{\left(\alpha_{1}+\mu_{A}+d_{1}^{r}\right)\left(\gamma_{2}+\mu_{A}+d_{2}^{v}\right)-\alpha_{1} \gamma_{2}} \tau \rho \beta^{T} \frac{\gamma_{2} \hat{N}_{2}^{v}}{\gamma_{2} \hat{N}_{2}^{v}+\alpha_{2} \hat{N}_{2}^{r}}\right) .
\end{aligned}
$$

Next, we go through the possible scenarios in case (B). In case (b1), the expected duration of infection of an individual in class $I_{1}^{v}$ after the $(2 n+1)$ th trip $(n=0,1, \ldots)$ is

$$
\frac{\gamma_{2}}{\gamma_{2}+\mu_{I}+\delta+d_{2}^{v}}\left(\frac{\alpha_{1}}{\alpha_{1}+\mu_{I}+\delta+d_{1}^{r}} \frac{\gamma_{2}}{\gamma_{2}+\mu_{I}+\delta+d_{2}^{v}}\right)^{n} \frac{1}{\mu_{I}+\alpha_{1}+\delta+d_{1}^{r}}
$$

thus the total expected infection time can be obtained as

$$
\begin{gathered}
\frac{\gamma_{2}}{\gamma_{2}+\mu_{I}+\delta+d_{2}^{v}} \sum_{n=0}^{\infty}\left(\frac{\alpha_{1}}{\alpha_{1}+\mu_{I}+\delta+d_{1}^{r}} \frac{\gamma_{2}}{\gamma_{2}+\mu_{I}+\delta+d_{2}^{v}}\right)^{n} \frac{1}{\alpha_{1}+\mu_{I}+\delta+d_{1}^{r}} \\
=\frac{\gamma_{2}}{\left(\alpha_{1}+\mu_{I}+\delta+d_{1}^{r}\right)\left(\gamma_{2}+\mu_{I}+\delta+d_{2}^{v}\right)-\alpha_{1} \gamma_{2}},
\end{gathered}
$$

and the number of new infections in case (b1) is

$$
\begin{aligned}
p & \left(\frac{\mu_{E} \alpha_{1}}{\left(\alpha_{1}+\mu_{E}+d_{1}^{r}\right)\left(\gamma_{2}+\mu_{E}+d_{2}^{v}\right)-\alpha_{1} \gamma_{2}}\right. \\
& \left.\cdot \frac{\gamma_{2}}{\left(\alpha_{1}+\mu_{I}+\delta+d_{1}^{r}\right)\left(\gamma_{2}+\mu_{I}+\delta+d_{2}^{v}\right)-\alpha_{1} \gamma_{2}} \beta_{1}^{r r} \frac{\hat{N}_{1}^{r}}{\hat{N}_{1}^{r}+\hat{N}_{1}^{v}}\right) \\
+(1-p) & \left(\frac{\mu_{E} \alpha_{1}}{\left(\alpha_{1}+\mu_{E}+d_{1}^{r}\right)\left(\gamma_{2}+\mu_{E}+d_{2}^{v}\right)-\alpha_{1} \gamma_{2}}\right. \\
& \left.\cdot \frac{\gamma_{2}}{\left(\alpha_{1}+\mu_{A}+d_{1}^{r}\right)\left(\gamma_{2}+\mu_{A}+d_{2}^{v}\right)-\alpha_{1} \gamma_{2}} \rho \beta_{1}^{r r} \frac{\hat{N}_{1}^{r}}{\hat{N}_{1}^{r}+\hat{N}_{1}^{v}}\right) .
\end{aligned}
$$


Similarly, the probability of being (symptomatic) infected just before the $(2 n+1)$ th trip $(n=0,1, \ldots)$ in case $(\mathrm{b} 2)$ is

$$
\left(\frac{\gamma_{2}}{\gamma_{2}+\mu_{I}+\delta+d_{2}^{v}} \frac{\alpha_{1}}{\alpha_{1}+\mu_{I}+\delta+d_{1}^{r}}\right)^{n} \frac{\gamma_{2}}{\gamma_{2}+\mu_{I}+\delta+d_{2}^{v}}
$$

moreover the duration of the infectious period during the $(2 n+1)$ th trip is $\tau$. Thus it follows that the total expected infection time of a symptomatic infected individual in case (b2) is

$$
\begin{gathered}
\sum_{n=0}^{\infty} \tau \\
\left.\quad=\frac{\gamma_{2}}{\gamma_{2}+\mu_{I}+\delta+d_{2}^{v}} \frac{\alpha_{1}}{\alpha_{1}+\mu_{I}+\delta+d_{1}^{r}}\right)^{n} \frac{\gamma_{2}}{\gamma_{2}+\mu_{I}+\delta+d_{2}^{v}} \\
\left(\alpha_{1}+\mu_{I}+\delta+\gamma_{2}\left(\alpha_{1}+\mu_{I}+\delta+d_{1}^{r}\right)\right.
\end{gathered}
$$

and we obtain the number of new infections in case (b2) as

$$
\begin{aligned}
p & \left(\frac{\mu_{E} \alpha_{1}}{\left(\alpha_{1}+\mu_{E}+d_{1}^{r}\right)\left(\gamma_{2}+\mu_{E}+d_{2}^{v}\right)-\alpha_{1} \gamma_{2}}\right. \\
& \left.\cdot \frac{\gamma_{2}\left(\alpha_{1}+\mu_{I}+\delta+d_{1}^{r}\right)}{\left(\alpha_{1}+\mu_{I}+\delta+d_{1}^{r}\right)\left(\gamma_{2}+\mu_{I}+\delta+d_{2}^{v}\right)-\alpha_{1} \gamma_{2}} \tau \beta^{T} \frac{\gamma_{2} \hat{N}_{2}^{v}}{\gamma_{2} \hat{N}_{2}^{v}+\alpha_{2} \hat{N}_{2}^{r}}\right) \\
+(1-p) & \left(\frac{\mu_{E} \alpha_{1}}{\left(\alpha_{1}+\mu_{E}+d_{1}^{r}\right)\left(\gamma_{2}+\mu_{E}+d_{2}^{v}\right)-\alpha_{1} \gamma_{2}}\right. \\
& \left.\cdot \frac{\gamma_{2}\left(\alpha_{1}+\mu_{A}+d_{1}^{r}\right)}{\left(\alpha_{1}+\mu_{A}+d_{1}^{r}\right)\left(\gamma_{2}+\mu_{A}+d_{2}^{v}\right)-\alpha_{1} \gamma_{2}} \tau \rho \beta^{T} \frac{\gamma_{2} \hat{N}_{2}^{v}}{\gamma_{2} \hat{N}_{2}^{v}+\alpha_{2} \hat{N}_{2}^{r}}\right) .
\end{aligned}
$$

We arrive at the formula for $R_{11}^{r r}$ by summing the number of new infections in the different cases. 


$$
\begin{aligned}
R_{11}^{r r}= & p\left(\frac{\mu_{E}}{\left(\alpha_{1}+\mu_{E}+d_{1}^{r}\right)\left(\gamma_{2}+\mu_{E}+d_{2}^{v}\right)-\alpha_{1} \gamma_{2}}\right. \\
& \cdot \frac{\left(\gamma_{2}+\mu_{E}+d_{2}^{v}\right)\left(\gamma_{2}+\mu_{I}+\delta+d_{2}^{v}\right)+\alpha_{1} \gamma_{2}}{\left(\alpha_{1}+\mu_{I}+\delta+d_{1}^{r}\right)\left(\gamma_{2}+\mu_{I}+\delta+d_{2}^{v}\right)-\alpha_{1} \gamma_{2}} \beta_{1}^{r r} \frac{\hat{N}_{1}^{r}}{\hat{N}_{1}^{r}+\hat{N}_{1}^{v}} \\
& +\frac{\mu_{E} \alpha_{1} \gamma_{2}}{\left(\alpha_{1}+\mu_{E}+d_{1}^{r}\right)\left(\gamma_{2}+\mu_{E}+d_{2}^{v}\right)-\alpha_{1} \gamma_{2}} \\
& \left.\cdot \frac{\gamma_{2}+\mu_{E}+d_{2}^{v}+\alpha_{1}+\mu_{I}+\delta+d_{1}^{r}}{\left(\alpha_{1}+\mu_{I}+\delta+d_{1}^{r}\right)\left(\gamma_{2}+\mu_{I}+\delta+d_{2}^{v}\right)-\alpha_{1} \gamma_{2}} \tau \beta^{T} \frac{\gamma_{2} \hat{N}_{2}^{v}}{\gamma_{2} \hat{N}_{2}^{v}+\alpha_{2} \hat{N}_{2}^{r}}\right) \\
& +(1-p)\left(\frac{\mu_{E}}{\left(\alpha_{1}+\mu_{E}+d_{1}^{r}\right)\left(\gamma_{2}+\mu_{E}+d_{2}^{v}\right)-\alpha_{1} \gamma_{2}}\right. \\
& \cdot \frac{\left(\gamma_{2}+\mu_{E}+d_{2}^{v}\right)\left(\gamma_{2}+\mu_{A}+d_{2}^{v}\right)+\alpha_{1} \gamma_{2}}{\left(\alpha_{1}+\mu_{A}+d_{1}^{r}\right)\left(\gamma_{2}+\mu_{A}+d_{2}^{v}\right)-\alpha_{1} \gamma_{2}} \rho \frac{\mu_{1}^{r r}}{\hat{N}_{1}^{r}+\hat{N}_{1}^{v}} \\
& +\frac{\mu_{1} \gamma_{2}}{\left(\alpha_{1}+\mu_{E}+d_{1}^{r}\right)\left(\gamma_{2}+\mu_{E}+d_{2}^{v}\right)-\alpha_{1} \gamma_{2}} \\
& \left.\cdot \frac{\gamma_{2}+\mu_{E}+d_{2}^{v}+\alpha_{1}+\mu_{A}+d_{1}^{r}}{\left(\alpha_{1}+\mu_{A}+d_{1}^{r}\right)\left(\gamma_{2}+\mu_{A}+d_{2}^{v}\right)-\alpha_{1} \gamma_{2}} \tau \rho \beta^{T} \frac{\gamma_{2} \hat{N}_{2}^{v}}{\gamma_{2} \hat{N}_{2}^{v}+\alpha_{2} \hat{N}_{2}^{r}}\right) .
\end{aligned}
$$

We detail the calculation of another element $R_{12}^{r v}$, which is the number of new infections in $E_{2}^{v}$ originated from $E_{1}^{r}$. We may define the two scenarios for the exposed-to-infected transition as before, and thus the above calculated probabilities for events (A) and (B) still hold.

$$
\begin{aligned}
& P(A)=\frac{\mu_{E}\left(\gamma_{2}+\mu_{E}+d_{2}^{v}\right)}{\left(\alpha_{1}+\mu_{E}+d_{1}^{r}\right)\left(\gamma_{2}+\mu_{E}+d_{2}^{v}\right)-\alpha_{1} \gamma_{2}}, \\
& P(B)=\frac{\mu_{E} \alpha_{1}}{\left(\alpha_{1}+\mu_{E}+d_{1}^{r}\right)\left(\gamma_{2}+\mu_{E}+d_{2}^{v}\right)-\alpha_{1} \gamma_{2}} .
\end{aligned}
$$

Again, in each case different ways of disease transmission arise. In case (A), an infected individual can transmit the disease as follows:

(a1) after an even number of completed trips (counted since the individual became infected), the individual is a member of class $I_{1}^{r}\left(A_{1}^{r}\right)$; the individual leaves region 1 and infects during travel from region 1 to region 2 , or

(a2) as a member of class $I_{2}^{v}\left(A_{2}^{v}\right)$, i.e., the individual infects in region 2 after an odd number of completed trips (counted since the individual became infected).

Similarly, in case (B), the individual can transmit the disease in one of the following ways:

(b1) after an odd number of completed trips (counted since the individual became infected), the individual is a member of class $I_{1}^{r}\left(A_{1}^{r}\right)$; the individual leaves region 1 and infects during travel from region 1 to region 2 , or 
(b2) as a member of class $I_{2}^{v}\left(A_{2}^{v}\right)$, i.e., the individual infects in region 2 after an even number of completed trips (counted since the individual became infected).

The total infection time in case (a1), (a2), (b1) and (b2) can be obtained with very similar calculations as by the element $R_{11}^{r r}$; that is, since the time of infection during travel is $\tau$, the total infection time of a symptomatic infected individual in case (a1) and (b1) is

$$
\begin{gathered}
\sum_{n=0}^{\infty} \tau\left(\frac{\alpha_{1}}{\alpha_{1}+\mu_{I}+\delta+d_{1}^{r}} \frac{\gamma_{2}}{\gamma_{2}+\mu_{I}+\delta+d_{2}^{v}}\right)^{n} \frac{\alpha_{1}}{\alpha_{1}+\mu_{I}+\delta+d_{1}^{r}} \\
=\frac{\tau \alpha_{1}\left(\gamma_{2}+\mu_{I}+\delta+d_{2}^{v}\right)}{\left(\alpha_{1}+\mu_{I}+\delta+d_{1}^{r}\right)\left(\gamma_{2}+\mu_{I}+\delta+d_{2}^{v}\right)-\alpha_{1} \gamma_{2}}
\end{gathered}
$$

and

$$
\begin{aligned}
\sum_{n=0}^{\infty} \tau & \frac{\gamma_{2}}{\gamma_{2}+\mu_{I}+\delta+d_{2}^{v}}\left(\frac{\gamma_{2}}{\gamma_{2}+\mu_{I}+\delta+d_{2}^{v}} \frac{\alpha_{1}}{\alpha_{1}+\mu_{I}+\delta+d_{1}^{r}}\right)^{n} \frac{\alpha_{1}}{\alpha_{1}+\mu_{I}+\delta+d_{1}^{r}} \\
& =\frac{\tau \alpha_{1} \gamma_{2}}{\left(\alpha_{1}+\mu_{I}+\delta+d_{1}^{r}\right)\left(\gamma_{2}+\mu_{I}+\delta+d_{2}^{v}\right)-\alpha_{1} \gamma_{2}}
\end{aligned}
$$

respectively, the duration of infection in case (a2) and (b2) is

$$
\begin{aligned}
& \sum_{n=0}^{\infty}\left(\frac{\alpha_{1}}{\alpha_{1}+\mu_{I}+\delta+d_{1}^{r}} \frac{\gamma_{2}}{\gamma_{2}+\mu_{I}+\delta+d_{2}^{v}}\right)^{n} \frac{\alpha_{1}}{\alpha_{1}+\mu_{I}+\delta+d_{1}^{r}} \cdot \frac{1}{\gamma_{2}+\mu_{I}+\delta+d_{2}^{v}} \\
& \quad=\frac{\alpha_{1}}{\left(\alpha_{1}+\mu_{I}+\delta+d_{1}^{r}\right)\left(\gamma_{2}+\mu_{I}+\delta+d_{2}^{v}\right)-\alpha_{1} \gamma_{2}}
\end{aligned}
$$

and

$$
\begin{aligned}
& \sum_{n=0}^{\infty}\left(\frac{\gamma_{2}}{\gamma_{2}+\mu_{I}+\delta+d_{2}^{v}} \frac{\alpha_{1}}{\alpha_{1}+\mu_{I}+\delta+d_{1}^{r}}\right)^{n} \frac{1}{\gamma_{2}+\mu_{I}+\delta+d_{2}^{v}} \\
& \quad=\frac{\alpha_{1}+\mu_{I}+\delta+d_{1}^{r}}{\left(\alpha_{1}+\mu_{I}+\delta+d_{1}^{r}\right)\left(\gamma_{2}+\mu_{I}+\delta+d_{2}^{v}\right)-\alpha_{1} \gamma_{2}} .
\end{aligned}
$$

Thus, the number of new infections from cases (a1) and (b1) are determined by the formulas

$$
\begin{aligned}
p & \left(\frac{\mu_{E}\left(\gamma_{2}+\mu_{E}+d_{2}^{v}\right)}{\left(\alpha_{1}+\mu_{E}+d_{1}^{r}\right)\left(\gamma_{2}+\mu_{E}+d_{2}^{v}\right)-\alpha_{1} \gamma_{2}}\right. \\
& \left.\cdot \frac{\alpha_{1}\left(\gamma_{2}+\mu_{I}+\delta+d_{2}^{v}\right)}{\left(\alpha_{1}+\mu_{I}+\delta+d_{1}^{r}\right)\left(\gamma_{2}+\mu_{I}+\delta+d_{2}^{v}\right)-\alpha_{1} \gamma_{2}} \tau \beta^{T} \frac{\alpha_{1} \hat{N}_{1}^{r}}{\alpha_{1} \hat{N}_{1}^{r}+\gamma_{1} \hat{N}_{1}^{v}}\right) \\
+(1-p) & \left(\frac{\mu_{E}\left(\gamma_{2}+\mu_{E}+d_{2}^{v}\right)}{\left(\alpha_{1}+\mu_{E}+d_{1}^{r}\right)\left(\gamma_{2}+\mu_{E}+d_{2}^{v}\right)-\alpha_{1} \gamma_{2}}\right. \\
& \left.\cdot \frac{\alpha_{1}\left(\gamma_{2}+\mu_{A}+d_{2}^{v}\right)}{\left(\alpha_{1}+\mu_{A}+d_{1}^{r}\right)\left(\gamma_{2}+\mu_{A}+d_{2}^{v}\right)-\alpha_{1} \gamma_{2}} \tau \rho \beta^{T} \frac{\alpha_{1} \hat{N}_{1}^{r}}{\alpha_{1} \hat{N}_{1}^{r}+\gamma_{1} \hat{N}_{1}^{v}}\right)
\end{aligned}
$$


and

$$
\begin{aligned}
p & \left(\frac{\mu_{E} \alpha_{1}}{\left(\alpha_{1}+\mu_{E}+d_{1}^{r}\right)\left(\gamma_{2}+\mu_{E}+d_{2}^{v}\right)-\alpha_{1} \gamma_{2}}\right. \\
& \left.\cdot \frac{\alpha_{1} \gamma_{2}}{\left(\alpha_{1}+\mu_{I}+\delta+d_{1}^{r}\right)\left(\gamma_{2}+\mu_{I}+\delta+d_{2}^{v}\right)-\alpha_{1} \gamma_{2}} \tau \beta^{T} \frac{\alpha_{1} \hat{N}_{1}^{r}}{\gamma_{1} \hat{N}_{1}^{v}+\alpha_{1} \hat{N}_{1}^{r}}\right) \\
+(1-p) & \left(\frac{\mu_{E} \alpha_{1}}{\left(\alpha_{1}+\mu_{E}+d_{1}^{r}\right)\left(\gamma_{2}+\mu_{E}+d_{2}^{v}\right)-\alpha_{1} \gamma_{2}}\right. \\
& \left.\cdot \frac{\alpha_{1} \gamma_{2}}{\left(\alpha_{1}+\mu_{A}+d_{1}^{r}\right)\left(\gamma_{2}+\mu_{A}+d_{2}^{v}\right)-\alpha_{1} \gamma_{2}} \tau \rho \beta^{T} \frac{\alpha_{1} \hat{N}_{1}^{r}}{\gamma_{1} \hat{N}_{1}^{v}+\alpha_{1} \hat{N}_{1}^{r}}\right) .
\end{aligned}
$$

We derive the number of new infections in cases (a2) and (b2) as

$$
\begin{aligned}
p & \left(\frac{\mu_{E}\left(\gamma_{2}+\mu_{E}+d_{2}^{v}\right)}{\left(\alpha_{1}+\mu_{E}+d_{1}^{r}\right)\left(\gamma_{2}+\mu_{E}+d_{2}^{v}\right)-\alpha_{1} \gamma_{2}}\right. \\
& \left.\cdot \frac{\alpha_{1}}{\left(\alpha_{1}+\mu_{I}+\delta+d_{1}^{r}\right)\left(\gamma_{2}+\mu_{I}+\delta+d_{2}^{v}\right)-\alpha_{1} \gamma_{2}} \beta_{2}^{v v} \frac{\hat{N}_{2}^{v}}{\hat{N}_{2}^{r}+\hat{N}_{2}^{v}}\right) \\
+(1-p) & \left(\frac{\mu_{E}\left(\gamma_{2}+\mu_{E}+d_{2}^{v}\right)}{\left(\alpha_{1}+\mu_{E}+d_{1}^{r}\right)\left(\gamma_{2}+\mu_{E}+d_{2}^{v}\right)-\alpha_{1} \gamma_{2}}\right. \\
& \left.\cdot \frac{\alpha_{1}}{\left(\alpha_{1}+\mu_{A}+d_{1}^{r}\right)\left(\gamma_{2}+\mu_{A}+d_{2}^{v}\right)-\alpha_{1} \gamma_{2}} \rho \beta_{2}^{v v} \frac{\hat{N}_{2}^{v}}{\hat{N}_{2}^{r}+\hat{N}_{2}^{v}}\right)
\end{aligned}
$$

and

$$
\begin{aligned}
p & \left(\frac{\mu_{E} \alpha_{1}}{\left(\alpha_{1}+\mu_{E}+d_{1}^{r}\right)\left(\gamma_{2}+\mu_{E}+d_{2}^{v}\right)-\alpha_{1} \gamma_{2}}\right. \\
& \left.\cdot \frac{\alpha_{1}+\mu_{I}+\delta+d_{1}^{r}}{\left(\alpha_{1}+\mu_{I}+\delta+d_{1}^{r}\right)\left(\gamma_{2}+\mu_{I}+\delta+d_{2}^{v}\right)-\alpha_{1} \gamma_{2}} \beta_{2}^{v v} \frac{\hat{N}_{2}^{v}}{\hat{N}_{2}^{r}+\hat{N}_{2}^{v}}\right) \\
+(1-p) & \left(\frac{\mu_{E} \alpha_{1}}{\left(\alpha_{1}+\mu_{E}+d_{1}^{r}\right)\left(\gamma_{2}+\mu_{E}+d_{2}^{v}\right)-\alpha_{1} \gamma_{2}}\right. \\
& \left.\cdot \frac{\alpha_{1}+\mu_{A}+d_{1}^{r}}{\left(\alpha_{1}+\mu_{A}+d_{1}^{r}\right)\left(\gamma_{2}+\mu_{A}+d_{2}^{v}\right)-\alpha_{1} \gamma_{2}} \rho \beta_{2}^{v v} \frac{\hat{N}_{2}^{v}}{\hat{N}_{2}^{r}+\hat{N}_{2}^{v}}\right) .
\end{aligned}
$$

We obtain the new infections in $E_{2}^{v}$ generated by individuals in $E_{1}^{r}$ by the formula 


$$
\begin{aligned}
R_{12}^{r v}= & p\left(\frac{\mu_{E} \alpha_{1}}{\left(\alpha_{1}+\mu_{E}+d_{1}^{r}\right)\left(\gamma_{2}+\mu_{E}+d_{2}^{v}\right)-\alpha_{1} \gamma_{2}}\right. \\
& \cdot \frac{\left(\gamma_{2}+\mu_{E}+d_{2}^{v}\right)\left(\gamma_{2}+\mu_{I}+\delta+d_{2}^{v}\right)+\alpha_{1} \gamma_{2}}{\left(\alpha_{1}+\mu_{I}+\delta+d_{1}^{r}\right)\left(\gamma_{2}+\mu_{I}+\delta+d_{2}^{v}\right)-\alpha_{1} \gamma_{2}} \tau \beta^{T} \frac{\alpha_{1} \hat{N}_{1}^{r}}{\gamma_{1} \hat{N}_{1}^{v}+\alpha_{1} \hat{N}_{1}^{r}} \\
& +\frac{\mu_{E} \alpha_{1}}{\left(\alpha_{1}+\mu_{E}+d_{1}^{r}\right)\left(\gamma_{2}+\mu_{E}+d_{2}^{v}\right)-\alpha_{1} \gamma_{2}} \\
& \left.\cdot \frac{\gamma_{2}+\mu_{E}+d_{2}^{v}+\alpha_{1}+\mu_{I}+\delta+d_{1}^{r}}{\left(\alpha_{1}+\mu_{I}+\delta+d_{1}^{r}\right)\left(\gamma_{2}+\mu_{I}+\delta+d_{2}^{v}\right)-\alpha_{1} \gamma_{2}} \beta_{2}^{v v} \frac{\hat{N}_{2}^{v}}{\hat{N}_{2}^{r}+\hat{N}_{2}^{v}}\right) \\
& +(1-p)\left(\frac{\mu_{1}}{\left(\alpha_{1}+\mu_{E}+d_{1}^{r}\right)\left(\gamma_{2}+\mu_{E}+d_{2}^{v}\right)-\alpha_{1} \gamma_{2}}\right. \\
& \cdot \frac{\left(\gamma_{2}+\mu_{E}+d_{2}^{v}\right)\left(\gamma_{2}+\mu_{A}+d_{2}^{v}\right)+\alpha_{1} \gamma_{2}}{\left(\alpha_{1}+\mu_{A}+d_{1}^{r}\right)\left(\gamma_{2}+\mu_{A}+d_{2}^{v}\right)-\alpha_{1} \gamma_{2}} \tau \beta^{T} \frac{\alpha_{1} \hat{N}_{1}^{r}}{\alpha_{1} \hat{N}_{1}^{r}+\gamma_{1} \hat{N}_{1}^{v}} \\
& +\frac{\mu_{1}}{\left(\alpha_{1}+\mu_{E}+d_{1}^{r}\right)\left(\gamma_{2}+\mu_{E}+d_{2}^{v}\right)-\alpha_{1} \gamma_{2}} \\
& \left.\cdot \frac{\gamma_{2}+\mu_{E}+d_{2}^{v}+\alpha_{1}+\mu_{A}+d_{1}^{r}}{\left(\alpha_{1}+\mu_{A}+d_{1}^{r}\right)\left(\gamma_{2}+\mu_{A}+d_{2}^{v}\right)-\alpha_{1} \gamma_{2}} \rho \beta_{2}^{v v} \frac{\hat{N}_{2}^{v}}{\hat{N}_{2}^{r}+\hat{N}_{2}^{v}}\right) .
\end{aligned}
$$

The element $R_{r v}^{11}$ can be obtained very similarly as $R_{r r}^{11}$ (change $S_{1}^{r}$ to $S_{1}^{v}$, change $\gamma_{2} S_{2}^{v}$ to $\alpha_{2} S_{2}^{r}$, change $\beta_{1}^{r r}$ to $\beta_{1}^{r v}$ ). $R_{r r}^{12}$ is derived as one writes $S_{2}^{r}$ instead of $S_{2}^{v}, \gamma_{1} S_{1}^{v}$ instead of $\alpha_{1} S_{1}^{r}$ and $\beta_{2}^{v r}$ instead of $\beta_{2}^{v v}$ in the formula of $R_{r v}^{12}$. The elements of the second column can be derived by using the elements of the first column and changing the first upper index of the transmission rates from $r$ to $v$ (i.e., $\beta_{1}^{v r}$ instead of $\beta_{1}^{r r}$ etc). For an element $R_{j, k}^{m, n}$ of the third or forth column one may consider $R_{k, j}^{m, n}$, the corresponding element which is in the first or second column, and change index 1 to 2 and index 2 to 1 .

Now we show that $\mathcal{K}=\mathcal{N}$. The first element of the first row of $\mathcal{K}$ is obtained as the scalar product of the first row of $\mathbb{F}$ and the first column of $\mathbb{V}^{-1}$. From earlier in this section we obtain

$$
\begin{array}{r}
(\mathbb{F})_{1, \cdot}=\left(0, \rho \beta_{1}^{r r} \frac{\hat{N}_{1}^{r}}{\hat{N}_{1}^{r}+\hat{N}_{1}^{v}}, \beta_{1}^{r r} \frac{\hat{N}_{1}^{r}}{\hat{N}_{1}^{r}+\hat{N}_{1}^{v}}, 0, \rho \beta_{1}^{v r} \frac{\hat{N}_{1}^{r}}{\hat{N}_{1}^{r}+\hat{N}_{1}^{v}}, \beta_{1}^{v r} \frac{\hat{N}_{1}^{r}}{\hat{N}_{1}^{r}+\hat{N}_{1}^{v}}, 0\right. \\
\left.\frac{\tau \beta^{T} \rho \alpha_{2} \gamma_{2} \hat{N}_{2}^{v}}{\alpha_{2} \hat{N}_{2}^{r}+\gamma_{2} \hat{N}_{2}^{v}}, \frac{\tau \beta^{T} \alpha_{2} \gamma_{2} \hat{N}_{2}^{v}}{\alpha_{2} \hat{N}_{2}^{r}+\gamma_{2} \hat{N}_{2}^{v}}, 0, \frac{\tau \beta^{T} \rho \gamma_{2}^{2} \hat{N}_{2}^{v}}{\alpha_{2} \hat{N}_{2}^{r}+\gamma_{2} \hat{N}_{2}^{v}}, \frac{\tau \beta^{T} \gamma_{2}^{2} \hat{N}_{2}^{v}}{\alpha_{2} \hat{N}_{2}^{r}+\gamma_{2} \hat{N}_{2}^{v}}\right)
\end{array}
$$




$$
\begin{aligned}
& \left(\mathbb{V}^{-1}\right)_{\cdot, 1}= \\
& \left(\frac{\gamma_{2}+\mu_{E}+d_{v}^{2}}{\left(\alpha_{1}+\mu_{E}+d_{r}^{1}\right)\left(\gamma_{2}+\mu_{E}+d_{v}^{2}\right)-\alpha_{1} \gamma_{2}},\right. \\
& \frac{(1-p) \mu_{E}}{\left(\alpha_{1}+\mu_{E}+d_{1}^{r}\right)\left(\gamma_{2}+\mu_{E}+d_{2}^{v}\right)-\alpha_{1} \gamma_{2}} \cdot \frac{\left(\gamma_{2}+\mu_{E}+d_{2}^{v}\right)\left(\gamma_{2}+\mu_{A}+d_{2}^{v}\right)+\alpha_{1} \gamma_{2}}{\left(\alpha_{1}+\mu_{A}+d_{1}^{r}\right)\left(\gamma_{2}+\mu_{A}+d_{2}^{v}\right)-\alpha_{1} \gamma_{2}}, \\
& \frac{p \mu_{E}}{\left(\alpha_{1}+\mu_{E}+d_{1}^{r}\right)\left(\gamma_{2}+\mu_{E}+d_{2}^{v}\right)-\alpha_{1} \gamma_{2}} \cdot \frac{\left(\gamma_{2}+\mu_{E}+d_{2}^{v}\right)\left(\gamma_{2}+\mu_{I}+\delta+d_{2}^{v}\right)+\alpha_{1} \gamma_{2}}{\left(\alpha_{1}+\mu_{I}+\delta+d_{1}^{r}\right)\left(\gamma_{2}+\mu_{I}+\delta+d_{2}^{v}\right)-\alpha_{1} \gamma_{2}}, \\
& 0,0,0,0,0,0, \frac{\alpha_{1}}{\left(\alpha_{1}+\mu_{E}+d_{r}^{1}\right)\left(\gamma_{2}+\mu_{E}+d_{v}^{2}\right)-\alpha_{1} \gamma_{2}}, \\
& \frac{(1-p) \mu_{E} \alpha_{1}}{\left(\alpha_{1}+\mu_{E}+d_{1}^{r}\right)\left(\gamma_{2}+\mu_{E}+d_{2}^{v}\right)-\alpha_{1} \gamma_{2}} \cdot \frac{\gamma_{2}+\mu_{E}+d_{2}^{v}+\alpha_{1}+\mu_{A}+d_{1}^{r}}{\left(\alpha_{1}+\mu_{A}+d_{1}^{r}\right)\left(\gamma_{2}+\mu_{A}+d_{2}^{v}\right)-\alpha_{1} \gamma_{2}}, \\
& \left.\frac{p \mu_{E} \alpha_{1}}{\left(\alpha_{1}+\mu_{E}+d_{1}^{r}\right)\left(\gamma_{2}+\mu_{E}+d_{2}^{v}\right)-\alpha_{1} \gamma_{2}} \cdot \frac{\gamma_{2}+\mu_{E}+d_{2}^{v}+\alpha_{1}+\mu_{I}+\delta+d_{1}^{r}}{\left(\alpha_{1}+\mu_{I}+\delta+d_{1}^{r}\right)\left(\gamma_{2}+\mu_{I}+\delta+d_{2}^{v}\right)-\alpha_{1} \gamma_{2}}\right)^{T} \text {, }
\end{aligned}
$$

and the product indeed equals $R_{11}^{r r}$. In order to obtain the first element of the forth row of $\mathcal{K}$, one needs to multiply the tenth row of $\mathbb{F}$ with the first column of $\mathbb{V}^{-1}$. The tenth row of $\mathbb{F}$ is

$$
\begin{aligned}
& (\mathbb{F})_{10, \cdot}=\left(0, \frac{\tau \beta^{T} \rho \alpha_{1}^{2} \hat{N}_{1}^{r}}{\alpha_{1} \hat{N}_{1}^{r}+\gamma_{1} \hat{N}_{1}^{v}}, \frac{\tau \beta^{T} \alpha_{1}^{2} \hat{N}_{1}^{r}}{\alpha_{1} \hat{N}_{1}^{r}+\gamma_{1} \hat{N}_{1}^{v}}, 0, \frac{\tau \beta^{T} \rho \alpha_{1} \gamma_{1} \hat{N}_{1}^{r}}{\alpha_{1} \hat{N}_{1}^{r}+\gamma_{1} \hat{N}_{1}^{v}}, \frac{\tau \beta^{T} \alpha_{1} \gamma_{1} \hat{N}_{1}^{r}}{\alpha_{1} \hat{N}_{1}^{r}+\gamma_{1} \hat{N}_{1}^{v}},\right. \\
& \left.0, \rho \beta_{2}^{r v} \frac{\hat{N}_{2}^{v}}{\hat{N}_{2}^{r}+\hat{N}_{2}^{v}}, \beta_{2}^{r v} \frac{\hat{N}_{2}^{v}}{\hat{N}_{2}^{r}+\hat{N}_{2}^{v}}, 0, \rho \beta_{2}^{v v} \frac{\hat{N}_{2}^{v}}{\hat{N}_{2}^{r}+\hat{N}_{2}^{v}}, \beta_{2}^{v v} \frac{\hat{N}_{2}^{v}}{\hat{N}_{2}^{r}+\hat{N}_{2}^{v}}\right) \text {, }
\end{aligned}
$$

the product indeed gives $R_{12}^{r v}$. The equalities of the other 14 elements of $\mathcal{K}$ and $\mathcal{N}$ arise similarly.

\subsubsection{The dependence of $\mathcal{R}_{0}$ on key model parameters}

Throughout this subsection we demonstrate how the reproduction number depends on various key model parameters. We previously described the calculation of $\mathcal{R}_{0}$ as the dominant eigenvalue of the next generation matrix. We obtained that each element of this $4 \times 4$ matrix is given by a complex formula of the model parameters, hence due to the complicated structure, we do not present here analytic results but numerical simulations with reasonable parameter values. We performed a systematic analysis to reveal the dependence of $\mathcal{R}_{0}$ on several model parameters. We chose reasonable values for the parameters (see Section 3.5 for plausible parameter ranges for influenza), population sizes and travel rates (see [24]). In this subsection we assume that the two regions are symmetric in the population sizes, travel rates and epidemiological characteristics. This assumption enables us to focus on a better understanding of the role of the key parameters. The findings support our intuitions about the dependence of the basic reproduction number on epidemiological 


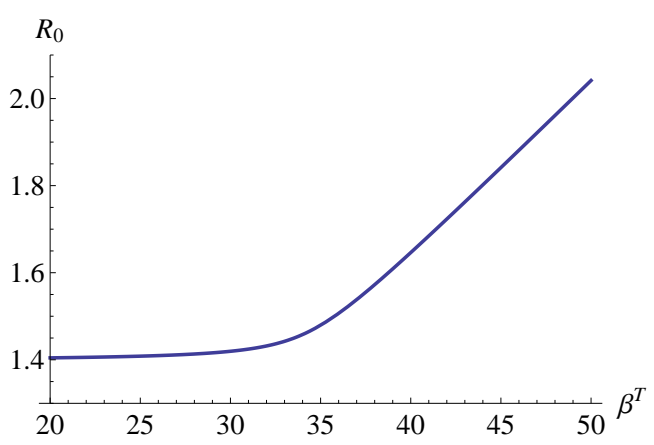

Figure 3.2: The dependence of $\mathcal{R}_{0}$ on $\beta^{T}$. Parameter values were chosen as $\mathcal{R}_{L, 1}=1.4$, $\mathcal{R}_{L, 2}=1.4, \tau=0.5, \gamma_{1}^{-1}=\gamma_{2}^{-1}=7, \mu_{E}^{-1}=1.4, \mu_{I}^{-1}=3, \mu_{A}^{-1}=4.1, p=0.6, \rho=0.1$.

parameters like the transmission rates and duration of infectious periods: increasing the values of these parameters results in an increase of the value of the reproduction number. However, we observed some unexpected behavior when examining $\mathcal{R}_{0}$ as a function of the transmission rate during travel and the travel rate of visitors.

Figure 3.2 shows $\mathcal{R}_{0}$ as a function of $\beta^{T}$ when other model parameters are fixed and the local reproduction numbers are set to 1.4. For $\beta^{T}<30$ the reproduction number settles at around 1.4, however the function approaches a line with a strictly positive slope as we further increase the parameter value. Numerical simulations proved that there is an eigenvalue of the NGM whose dependence on $\beta^{T}$ is almost linear. As the parameter value grows, this eigenvalue dominates the one which is close to 1.4 for each value of $\beta^{T}$. Next we set $\beta^{T}=40$ and examine the effect of parameters $\gamma_{1}$ and $\gamma_{2}$ on the reproduction number. If we ignore the time needed to complete a one-way trip, the value of these parameters does not influence the value of $\mathcal{R}_{0}$ in the case of identical regions: if $\tau=0$ and the two regions are symmetric in the population sizes and values of epidemiological parameters including the local reproduction numbers, then $\mathcal{R}_{0}=\mathcal{R}_{L, 1}=\mathcal{R}_{L, 2}$ holds. However, for positive values of $\tau$ we obtain some non-monotonic behavior of $\mathcal{R}_{0}$ as a function of the duration of visitors' stay (reciprocal of $\gamma_{1}$ and $\gamma_{2}$ ) as shown in Figure 3.3. To understand this phenomenon we examined how the elements of the next generation matrix depend on $\frac{1}{\gamma_{1}}$ and $\frac{1}{\gamma_{2}}$. We found that these parameters do not significantly influence most of the matrix elements if one considers realistic parameter range $\left(0<\frac{1}{\gamma_{1}}, \frac{1}{\gamma_{2}}<50\right.$ (days)). However, $R_{21}^{v r}, R_{21}^{v v}, R_{12}^{v r}$ and $R_{12}^{v v}$ have similar non-monotonic shapes as obtained by the reproduction number.

See Figure 3.4 for the graph of four elements of the next generation matrix. The values of $R_{11}^{r r}$ and $R_{21}^{r r}$ do not depend strongly on the length of visitors' stay, as for real air traffic data travel rates of residents are low, hence with high probability the exposed resident never 


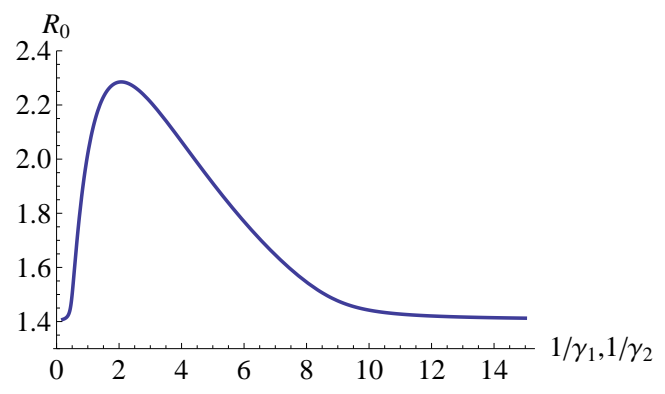

Figure 3.3: The dependence of $\mathcal{R}_{0}$ on $\frac{1}{\gamma_{1}}$ and $\frac{1}{\gamma_{2}}$ in the case of $\gamma_{1}=\gamma_{2}$ and $\tau>0$. Parameter values were chosen as $\mathcal{R}_{L, 1}=1.4, \mathcal{R}_{L, 2}=1.4, \tau=0.5, \beta^{T}=40, \mu_{E}^{-1}=1.4, \mu_{I}^{-1}=3$, $\mu_{A}^{-1}=4.1, p=0.6, \rho=0.1$.

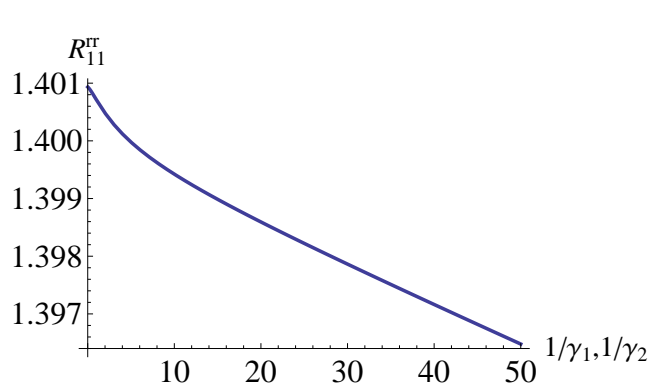

(a) $R_{11}^{r r}$

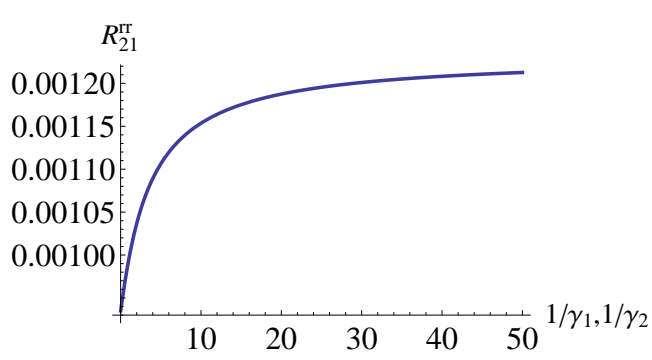

(c) $R_{21}^{r r}$

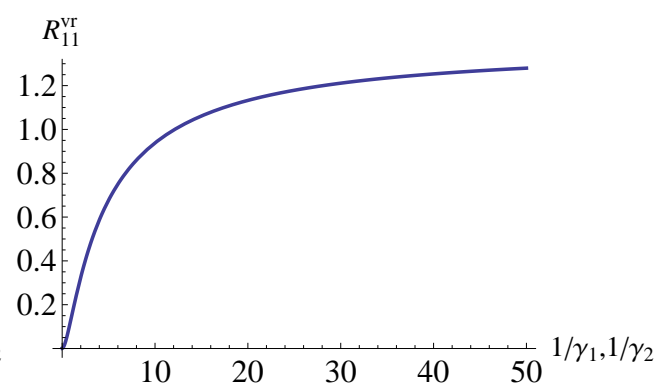

(b) $R_{11}^{v r}$

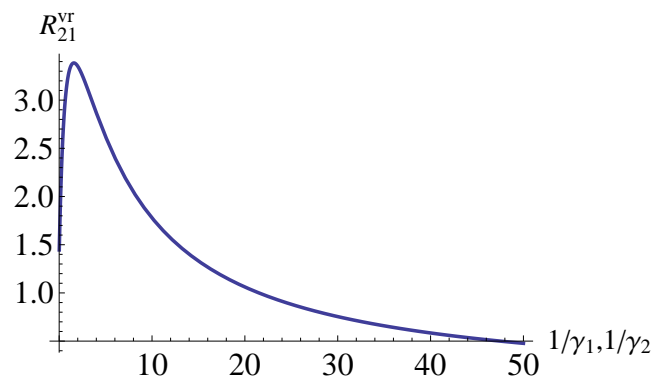

(d) $R_{21}^{v r}$

Figure 3.4: The dependence of some elements of the next generation matrix on $\frac{1}{\gamma_{1}}$ and $\frac{1}{\gamma_{2}}$ in the case of $\gamma_{1}=\gamma_{2}$ and $\tau>0$. Parameter values were chosen as $\mathcal{R}_{L, 1}=1.4, \mathcal{R}_{L, 2}=1.4$, $\tau=0.5, \beta^{T}=40, \mu_{E}^{-1}=1.4, \mu_{I}^{-1}=3, \mu_{A}^{-1}=4.1, p=0.6, \rho=0.1$.

becomes a visitor. The longer visitors stay in the foreign region on average, the higher $R_{11}^{v r}$ is (converges to $\mathcal{R}_{L, 1}$ as $\frac{1}{\gamma_{1}}$ and $\frac{1}{\gamma_{2}}$ tend to infinity). The element $R_{21}^{v r}$ defines the number of new infections among residents of region 1 caused by a single exposed visitor of region 2. Following our definition of reproduction, $R_{21}^{v r}$ counts new infections accrued while the visitor was traveling to region 1 and while the visitor was staying there. If the duration of visitors' stay is short, the probability that a visitor leaves region 2 before finishing his 


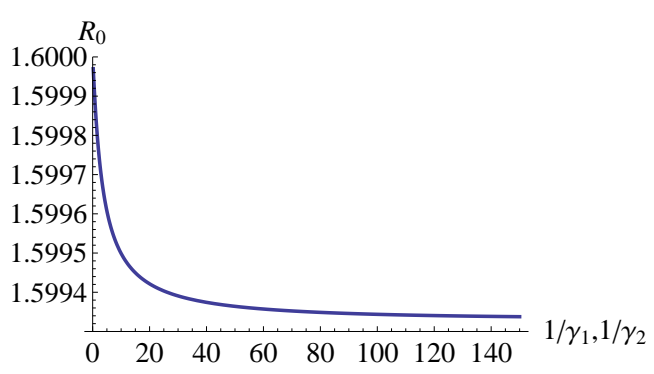

Figure 3.5: The dependence of $\mathcal{R}_{0}$ on $\frac{1}{\gamma_{1}}$ and $\frac{1}{\gamma_{2}}$ in the case of $\gamma_{1}=\gamma_{2}$ and $\tau=0$, i.e., we neglect the time required to complete a one-way trip. Parameter values were chosen as $\mathcal{R}_{L, 1}=1.2, \mathcal{R}_{L, 2}=1.6, \tau=0, \mu_{E}^{-1}=1.4, \mu_{I}^{-1}=3, \mu_{A}^{-1}=4.1, p=0.6, \rho=0.1$.

exposed period is high, and as we neglected the possibility of moving to classes $I$ or $A$ during travel, he will start his infectious period only in region 1. Choosing realistic values for travel rates makes the chance of leaving region 1 small, thus for small values of $\frac{1}{\gamma_{1}}$ and $\frac{1}{\gamma_{2}}, R_{21}^{v r}$ approximately equals 1.4 , the value of the local reproduction number $\mathcal{R}_{L, 1}$. As the length of visitors' stay increases, the chances for the visitor to move to class $I$ or $A$ before traveling back to region 1 rise. This results in the elevated number of newly infected individuals due to increased transmission potential during travel. However, if the duration of visitors' stay is much longer, the probability that the visitor travels back to region 1 and gets into contact with residents there (in region 1) is low, hence the expected number of such new infections is close to zero. The graphical interpretation of other elements of the NGM can be explained similarly. We remark that the non-monotonic behavior of the reproduction number as a function of the duration of visitors' stay is observed in the case of asymmetric regions as well. However, if we neglect the duration of travel and assume different local reproduction numbers in the regions, the graph of $\mathcal{R}_{0}$ becomes monotonically decreasing as shown in Figure 3.5. This shows that including travel-related infections can fundamentally change the way the reproduction number depends on various model parameters, and in the case of a non-monotone dependence on a parameter, one has to be very careful when proposing control measures which change the given parameter.

\subsection{3 $\mathcal{R}_{0}$ as the threshold quantity for epidemic outbreaks}

The importance of parameter $\beta^{T}$ in the computation of the reproduction number has been revealed in the previous subsection. However, it is not clear how the nonlinear dependence of $\mathcal{R}_{0}$ on the transmission rate during travel is reflected on the epidemic curves or the final epidemic size. We consider the hypothetical case when the two regions are symmetric in the population sizes, travel rates and values of every model parameter except the local reproduction numbers $\left(\mathcal{R}_{L, 1}=\mathcal{R}_{L, 2}\right.$ would result in identical epidemic curves). See Section 


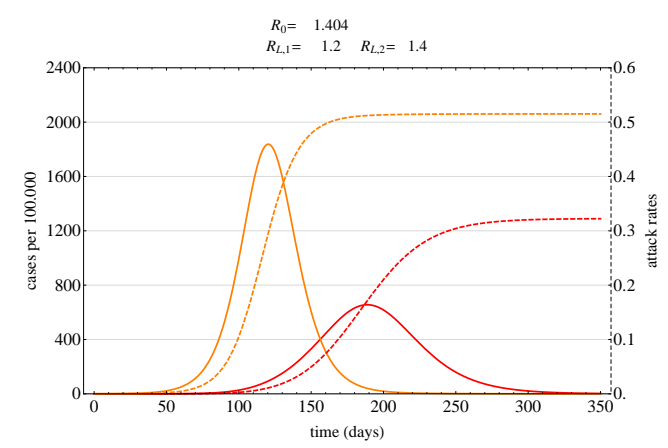

(a) Peak time in region 1: day 178, peak time in region 2: day 122 , when $\mathcal{R}_{L, 1}=1.2, \mathcal{R}_{L, 2}=$ $1.4, \beta^{T}=25$.

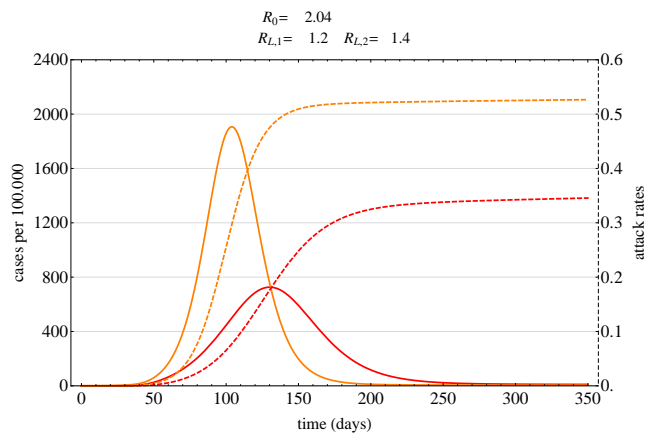

(b) Peak time in region 1: day 126, peak time in region 2: day 103 , when $\mathcal{R}_{L, 1}=1.2, \mathcal{R}_{L, 2}=$ $1.4, \beta^{T}=50$.

Figure 3.6: Epidemic curves and attack rates of region 1 (red and red-dashed) and region 2 (orange and orange-dashed) for two values of $\beta^{T}$ when both the local reproduction numbers are greater than 1 . The increase in the value of $\beta^{T}$ significantly alters the value of $\mathcal{R}_{0}$, but its effect on the epidemic curves only manifests in earlier peak times. Parameter values were chosen as $\mathcal{R}_{L, 1}=1.2, \mathcal{R}_{L, 2}=1.4, \tau=0.5, \mu_{E}^{-1}=1.4, \mu_{I}^{-1}=3, \mu_{A}^{-1}=4.1, p=0.6$, $\rho=0.1, \gamma_{1}^{-1}=\gamma_{2}^{-1}=7, \alpha_{1}=5 \cdot 10^{-5}, \alpha_{2}=5 \cdot 10^{-5}, N_{1}^{r}(0)=3.4 \cdot 10^{7}, N_{2}^{r}(0)=3.4 \cdot 10^{7}$.

3.5 for realistic travel rates, population sizes and epidemiological parameters for influenza.

If both the regional reproduction numbers are greater than one, increasing $\beta^{T}$ does not have a significant effect on the disease outbreak: although the curves peak earlier for larger values of the parameter, peak sizes and attack rates (defined as the fraction of individuals who have contracted the disease) remain similar in both regions. However, as illustrated in Figure 3.6, the difference manifests in the value of the basic reproduction number: changing $\beta^{T}$ from 25 to 50 can increase $\mathcal{R}_{0}$ from 1.4 for 2 in this particular case.

There is no epidemic outbreak in the absence of travel if we assume that both regional reproduction numbers are less than 1 . In case of connected regions, minor outbreaks can occur if the value of $\beta^{T}$ is set to ensure that $\mathcal{R}_{0}$ exceeds one. However, further increasing $\beta^{T}$ may result in long-continued outbreaks with small peak sizes but relatively high values of the reproduction number and the attack rates, as indicated in Figure 3.7. These examples clearly show that although - as we have proved it in Section $3.4-\mathcal{R}_{0}$ works as a threshold regarding the stability of the disease free state (that is relevant to the initial growth of an epidemic), it is not necessarily a good predictor for the entire course of the outbreak and the attack rates. 


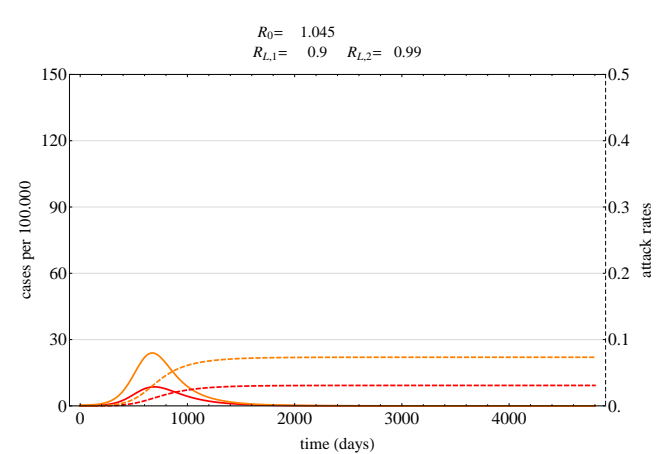

(a) $\mathcal{R}_{L, 1}=0.9, \mathcal{R}_{L, 2}=0.99, \beta^{T}=25$

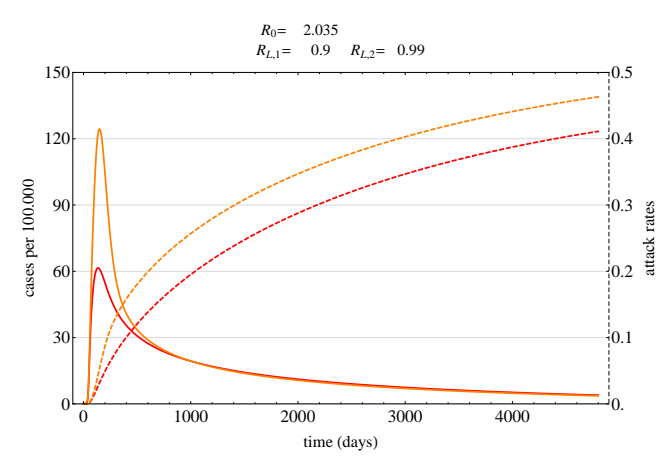

(b) $\mathcal{R}_{L, 1}=0.9, \mathcal{R}_{L, 2}=0.99, \beta^{T}=50$

Figure 3.7: Epidemic curves and attack rates of region 1 (red and red-dashed) and region 2 (orange and orange-dashed) for two values of $\beta^{T}$ when both the local reproduction numbers are below 1 but $\mathcal{R}_{0}>1$. The relatively high value of the reproduction number is reflected in the size of the outbreak: the dashed curves show that for $\beta^{T}=50$ more than $40 \%$ of the populations of both region 1 and 2 has been infected by day 4500. Parameter values were chosen as $\mathcal{R}_{L, 1}=0.9, \mathcal{R}_{L, 2}=0.99, \tau=0.5, \mu_{E}^{-1}=1.4, \mu_{I}^{-1}=3, \mu_{A}^{-1}=4.1, p=0.6$, $\rho=0.1, \gamma_{1}^{-1}=\gamma_{2}^{-1}=7, \alpha_{1}=5 \cdot 10^{-5}, \alpha_{2}=5 \cdot 10^{-5}, N_{1}^{r}(0)=3.4 \cdot 10^{7}, N_{2}^{r}(0)=3.4 \cdot 10^{7}$.

\subsection{Parametrization for influenza}

We parametrize our model for the $2009 \mathrm{~A}(\mathrm{H} 1 \mathrm{~N} 1)$ pandemic influenza. We ignore demography and set parameters $\Lambda_{j}, d_{j}^{r}$ and $d_{j}^{v}, j \in\{1,2\}$, equal to 0 , moreover we also neglect the possibility of disease induced mortality and let $\delta=0$. Several studies ([1, 4, 6, 9, 16, 30, $46,48,49]$ and the references therein) estimated the local reproduction number and values of key epidemiological parameters for recent influenza pandemics. In Table 3.3 we give an overview of the ranges of these parameters, and choose reasonable values from the ranges for our simulations. Parameter $\beta^{T}$, the transmission rate during travel is estimated to be 10-20, as [49] claims that the expected number of H1N1 infections caused by a single infectious case varies between 5 and 10, considering transmission during an eleven-hour-long flight.

In the model description, several parameters were introduced to characterize transportation between the regions: travel rate of residents from their origin, duration of visitors' stay, duration of travel. We determine the values of these parameters for specific cases: we pick Canada to be region 1 (origin) and we consider three possible destinations as region 2 (for the destinations, see Section 3.6). We derive travel rates from [24], which provides the annual volume of passengers toward Canada from several international sources. However, neither records about travelers' origin (Canadian resident or visitor of Canada), nor data 


\begin{tabular}{|c||c|cl|}
\hline Parameter & Value for simulations & Range from literature \\
\hline$\Lambda_{j}, d_{j}^{m}, \delta$ & 0 & & \\
$\mu_{E}^{-1}$ & 1.4 & $(1-2.62)$ & {$[4,46,48]$} \\
$\mu_{I}^{-1}$ & 3 & $(1.1-4.69)$ & {$[4,46,48]$} \\
$\mu_{A}^{-1}$ & 4.1 & $(2.06-4.69)$ & {$[30,46]$} \\
$\rho$ & 0.1 & $(0-0.5)$ & {$[1,6,30]$} \\
$p$ & 0.6 & $(0.5-0.75)$ & {$[1,9]$} \\
$\beta^{T}$ & 15 & $(10-20)$ & {$[16,49]$} \\
$\mathcal{R}_{L}$ & $1.3,1.4$ & & \\
$\beta_{j}^{m, n}$ & calculated from $\mathcal{R}_{L}$ & & \\
$\alpha_{j}, \gamma_{j}, \tau$ & see specific cases & & \\
\hline
\end{tabular}

Table 3.3: Parameters for simulations $(j \in\{1,2\}, m, n \in\{r, v\})$.

about the volume of passengers traveling in other directions are available in this study. For many destinations available from Canada, the surveys of Statistics Canada [40] provide information about the ratio of the volume of foreign travelers to Canada and Canadian travelers to the other region. We assume that all Canadian residents who leave Canada will return sometimes later, therefore for a specific region 2 , the ratio of the annual volume of residents of this region traveling to Canada and Canadians returning home from the region is explicitly given by the statistics. Thus, given the annual volume of all passengers from a specific region 2 toward Canada, we can determine $\Omega_{2}$, the annual volume of residents of region 2 traveling to Canada, and the annual volume of Canadian residents returning home from region 2. We assume that this number equals $\Omega_{1}$, the annual volume of Canadian passengers to region 2. The annual volume of passengers toward Canada and the ratio of Canadian residents and residents of region 2 traveling between Canada and region 2 for three origin-destination pairs can be found in Table 3.4.

We need $\alpha_{1}$ and $\alpha_{2}$, the traveling rates of Canadian residents and residents of region 2. In our simulations, we define the traveling rate of residents of region $j$ at time $t$ as $\alpha_{j}(t)=\frac{\Omega_{j}}{365} \frac{1}{N_{j}^{r}(t)}, j \in\{1,2\}$ (the definition is slightly different in the case of the origindestination pair "Canada-Mexico", see the corresponding subsection). Parameters $\gamma_{j}$ and 


\begin{tabular}{|c||c|c|}
\hline Origin-destination pair & Khan et al. [24] & Statistics Canada 2009 [40] \\
\hline Canada - China & 786569 & $9: 5$ \\
Canada - UK & 1203272 & $14: 11$ \\
Canada - Mexico & 655219 & $15: 2$ \\
\hline
\end{tabular}

Table 3.4: Annual travel volumes and statistics for three origin-destination pairs. The annual volumes of passengers toward Canada were derived from [24]. The statistics of [40] provide the ratio of Canadian residents and residents of the other region (China / United Kingdom / Mexico).

$\tau$ are determined for each specific origin-destination pair. Initial values are set as follows:

$$
\begin{aligned}
& S_{j}^{r}(u)= \begin{cases}\left(1-10^{-5}\right) M_{j} & \text { if } u=0, \\
M_{j} & \text { if } u<0,\end{cases} \\
& S_{j}^{v}(u) \equiv \frac{\Omega_{k}}{365 \gamma_{j}}, \\
& E_{j}^{r}(u)= \begin{cases}10^{-5} M_{j} & \text { if } u=0, \\
0 & \text { if } u<0,\end{cases}
\end{aligned}
$$

where $M_{j}$ denotes the population size of region $j$, and $E_{j}^{v}(u)=A_{j}^{m}(u)=I_{j}^{m}(u)=R_{j}^{m}(u) \equiv$ 0 for $u \in[-\tau, 0], j, k \in\{1,2\}, j \neq k, m \in\{r, v\}$. We determine the transmission rates $\beta_{j}^{m, n}, j \in\{1,2\}, m, n \in\{r, v\}$, as follows. For each origin-destination pair of our model, we denote the local reproduction number of region $j$ (i.e., the reproduction number of the region in the absence of travel) by $\mathcal{R}_{L, j}$. Several recent studies $([4,46])$ estimated the basic reproduction number for regions which were affected by the 2009 H1N1 pandemic. For a given $\mathcal{R}_{L, j}$, we can use the formula

$$
\mathcal{R}_{L, j}=\beta_{j} \frac{S_{j}^{r}(0)}{N_{j}^{r}(0)}\left(\frac{p}{\mu_{I}}+(1-p) \frac{\rho}{\mu_{A}}\right)
$$

to calculate $\beta_{j}$. Assuming homogeneous mixing in the regions, we can set $\beta_{j}^{r r}=\beta_{j}^{r v}=$ $\beta_{j}^{v r}=\beta_{j}^{v v}=\beta_{j}$. For the numerical simulations we set $\mu_{E}^{T}=\mu_{A}^{T}=\mu_{I}^{T}=0$; as pointed out in Section 3.4, this assumption allows us to obtain the inflow terms explicitly and also to calculate the reproduction number.

\subsection{Prototype origin-destination pairs}

In this section, we present simulations for influenza using real demographic and air traffic data. We set up three distinct scenarios for the origin-destination pairs: we choose Canada to represent region 1 and consider three possible geographic locations for region 2: China, Mexico and the United Kingdom. All these three countries are popular destinations of 
flights originated from Canada. We would like to emphasize that, although we used real demographic and air travel data in the simulations and chose reasonable parameter values from the ranges of Table 3.3, the epidemic curves depicted below do not need to match with the $2009 \mathrm{~A}(\mathrm{H} 1 \mathrm{~N} 1)$ influenza pandemic data reported, since there were other factors which are not considered in our model setting. The purpose of choosing such origin-destination pairs for simulations was to illustrate our model for regions parametrized with different values of the key model parameters. Nevertheless, we also present the data fitting results of the model to the Canadian and Mexican morbidity data of the first wave of the pandemic, since in the early stage these data were largely determined by the characteristics of the two countries and the travel between them. Table 3.4 summarizes real air traffic data and the ratios of Canadian residents traveling to region 2 and residents of region 2 traveling to Canada for three origin-destination pairs.

\subsubsection{Canada - China: The case of asymmetric populations}

China, including Hong Kong generates the third largest volume of international passenger traffic entering Canada: approximately 780000 air passengers initiate their trip from within China's borders. In aspect of global preparedness against worldwide spreading pandemics China is of particular interest: its variability of poverty and wealth provides a platform for the appearance of emerging infectious diseases (e.g., H5N1, SARS). The country's high connectivity with all parts of the world (it possesses the fifth largest international airport in the world) clearly shows it's significance in international spreading of the infection.

In the model construction we assumed that each individual of the population has equal chances to travel. This generalization is definitely not fulfilled in a population of more than 1 billion with various social-economic background, hence here we use a somewhat smaller population of potential travelers of China (150 million). The population size of Canada is set to 34.461 million, we let $\tau=0.5$ (days) since we assume that a flight between the regions takes approximately 12 hours. For the local reproduction numbers of Canada and China, we pick $\mathcal{R}_{L, 1}=1.3$ and $\mathcal{R}_{L, 2}=1$.4. Parameters $\alpha_{1}$ and $\alpha_{2}$ were derived using travel volumes as explained in Section 3.5, we choose $\gamma_{1}=\gamma_{2}=\frac{1}{7}$.

We present Figures 3.8 and 3.9 to demonstrate the role of the human transportation system played in the spread of influenza. Assuming that initial outbreaks in Canada (red curves) and China (blue curves) occurred independently, the effect of traveling on the spread of the epidemic seems negligible, because the epidemic curves in case of separated regions (Figure 3.8(a)) are very similar to the epidemic curves in case of connected regions (Figure 3.8(b)). However, Figure 3.8(c) clearly shows the importance of incorporating 


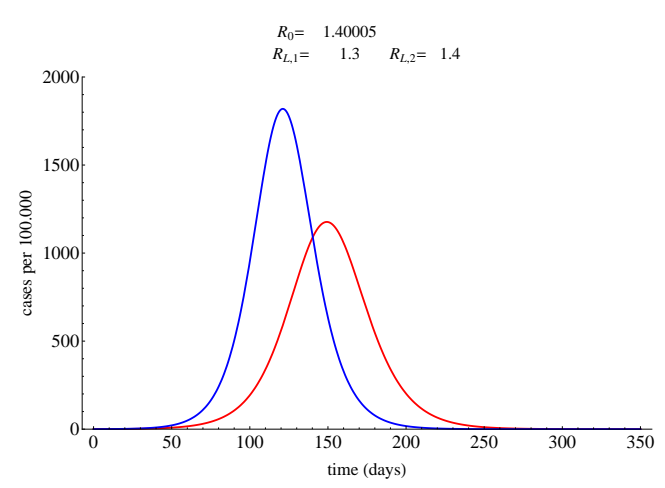

(a) Peak time in Canada: day 149, peak time in China: day 125 in the case of regions connected by air travel, independent outbreaks.

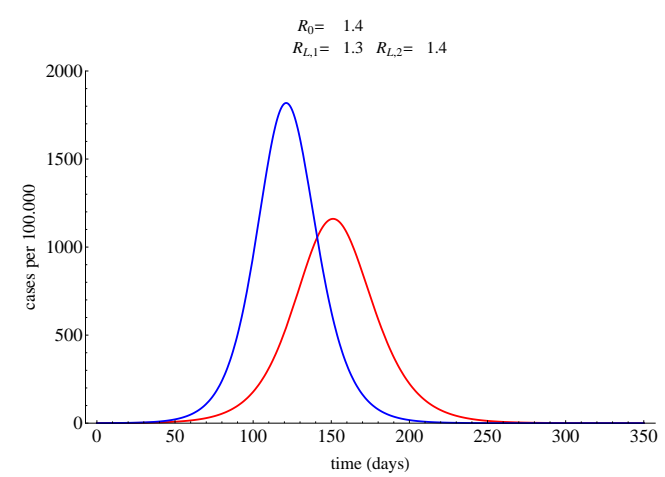

(b) Peak time in Canada: day 152, peak time in China: day 125 in the case of separated regions, independent outbreaks.

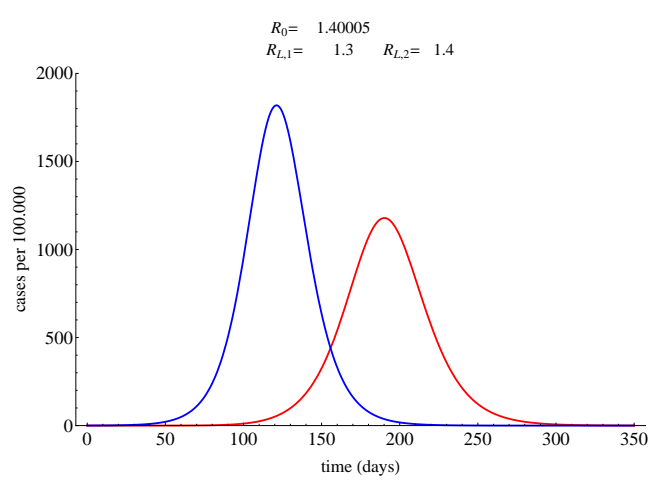

(c) Peak time in Canada: day 190, peak time in China: day 125 in the case of regions connected by air travel, initial cases only in China.

Figure 3.8: Epidemic curves of Canada (region 1, red) and China (region 2, blue). In case of independent outbreaks in Canada and China, the effect of travel on the spread of the pandemic is negligible. However, if we assume that the pandemic originates from China and Canada is susceptible, then due to air transportation the infection invades the disease free Canada, though with delayed peak time. For the simulations, we set $\mathcal{R}_{L, 1}=1.3$, $\mathcal{R}_{L, 2}=1.4, \tau=0.5, \gamma_{1}^{-1}=\gamma_{2}^{-1}=7, \beta^{T}=15, \mu_{E}^{-1}=1.4, \mu_{I}^{-1}=3, \mu_{A}^{-1}=4.1, p=0.6$, $\rho=0.1$.

transportation into the model: if we assume that initial cases only appear in China and Canada is completely susceptible, then the disease reaches Canada, obviously due to air transportation. This scenario results in approximately the same peak size as if we assumed initial outbreaks in both regions, although the peak time in Canada is delayed by almost 40 days. Analyzing the public reports of health agencies confirms that large delays between peak times of connected regions are unrealistic when one considers pandemics where air transportation was proved to play a key role in disease transmission (SARS 2002-2003, 


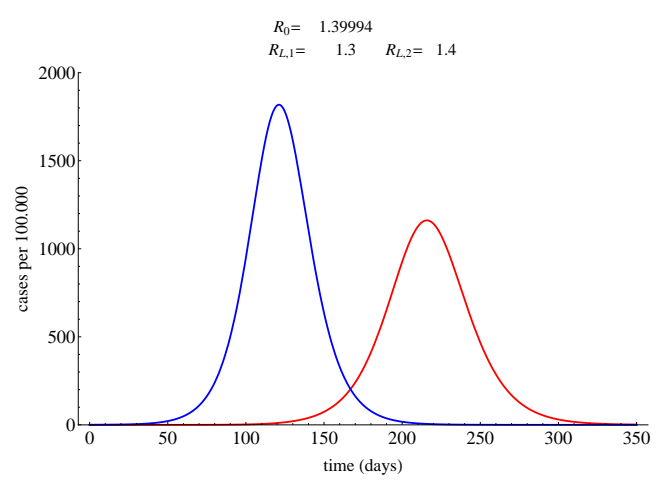

Figure 3.9: Epidemic curves of Canada (region 1, red) and China (region 2, blue) when we ignore the possibility of disease transmission during travel and we assume that the pandemic originates from China and Canada is susceptible. Peak time in Canada: day 220, peak time in China: day 125. For the simulations, we set $\mathcal{R}_{L, 1}=1.3, \mathcal{R}_{L, 2}=1.4$, $\tau=0.5, \gamma_{1}^{-1}=\gamma_{2}^{-1}=7, \beta^{T}=15, \mu_{E}^{-1}=1.4, \mu_{I}^{-1}=3, \mu_{A}^{-1}=4.1, p=0.6, \rho=0.1$.

$\mathrm{A}(\mathrm{H} 1 \mathrm{N1}) \mathrm{v}$ influenza 2009). Figure 3.9 shows what happens if we ignore the possibility of on-board disease transmission in the model. Comparing this result with Figure 3.8(c) is of particular interest: if one incorporates disease dynamics during travel, the model predicts the peak time of the invaded region to be 30 days earlier. As a concluding remark, we wish to emphasize that, following these findings, simpler models that ignore travelrelated infections can seriously overestimate the time a region has for preparation before the outbreak arrives.

\subsubsection{Canada - United Kingdom: The symmetric case}

The European Union generates almost one-fifth of all international traffic entering Canada. Although the EU may be an unlikely source for the emergence of new or dangerous infectious disease threats, it generates over $19 \%$ of the world's international traffic volume and consequently should receive special consideration as an important potential location from which infectious disease threats may enter Canada. Being the second leading international source of passenger traffic entering Canada (6.6 \%), the United Kingdom is an important international traffic intersection.

Unlike the Canada-China and the Canada-Mexico origin-destination pairs, the CanadaUnited Kingdom pair can be considered as the case of two symmetric regions. The population sizes of the two countries have the same magnitude, moreover just like Canada, the UK also possesses highly developed health care system and advanced intervention techniques in disease control and prevention, hence we can assume that the local reproduction numbers are similar. In the simulations we set the population size of the UK to be 62.262 


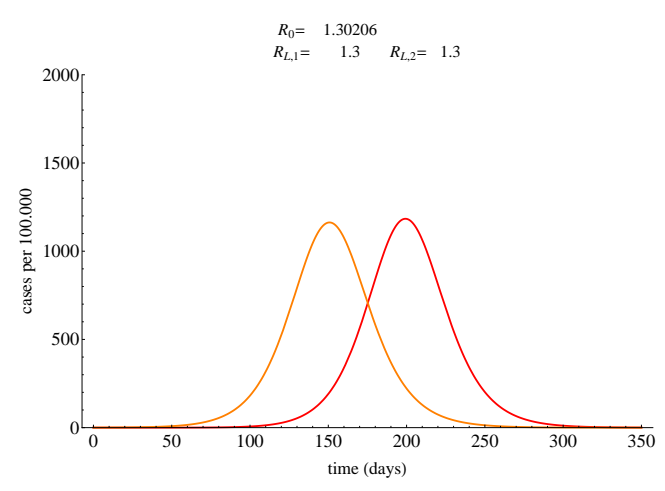

(a) Peak time in Canada: day 200, peak time in the UK: day 153 when there are no reduction in travel volumes.

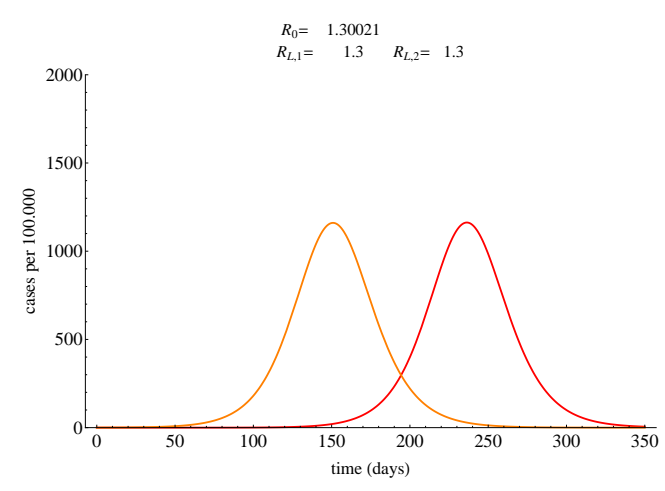

(b) Peak time in Canada: day 240, peak time in the UK: day 153 when there is a $90 \%$ reduction in travel volumes.

Figure 3.10: Reducing travel volumes by $90 \%$ between Canada (region 1, red) and the UK (region 2, orange) results in a delay of the peak time in Canada by 40 days. We assumed the initial number of infectious cases in Canada to be 0. For the simulations, we set $\mathcal{R}_{L, 1}=1.3, \mathcal{R}_{L, 2}=1.3, \tau=0.5, \gamma_{1}^{-1}=\gamma_{2}^{-1}=7, \beta^{T}=15, \mu_{E}^{-1}=1.4, \mu_{I}^{-1}=3$, $\mu_{A}^{-1}=4.1, p=0.6, \rho=0.1$.

million, $\mathcal{R}_{L, 1}=\mathcal{R}_{L, 2}=1.3, \gamma_{1}=\gamma_{2}=\frac{1}{7}$ and $\tau=0.5$.

Reducing the number of flights to and from infected areas and screening out infected individuals at their arrival to international airports are considered to be powerful tools when one's aim is to mitigate the severity of pandemic outbreaks. However, entry screening works ineffectively on asymptomatic infected individuals, who - despite their reduced disease transmissibility - can spread the infection after arrival. We examined the benefits of travel restrictions on the spread of the pandemic in two symmetric regions. We found that in the case of a single outbreak in the UK, the peak time of the outbreak in the originally disease free Canada can be delayed by 40 days when we consider the hypothetical case of $90 \%$ limitation in the travel volumes between the two regions. See Figure 3.10, red and orange curves show the number of symptomatic infected cases per 1000000 in Canada and the UK, respectively.

\subsubsection{Canada - Mexico: The case of asymmetric travel}

Mexico is a potential source of threatening pandemics due to high population density, poverty and limited health care resources on one side but high volume of international traffic on the other side. The country generates the forth largest volume of international passenger traffic entering Canada (approximately 655000 passengers, second largest volume from any developing countries). A significantly large part of this traffic originates from re- 


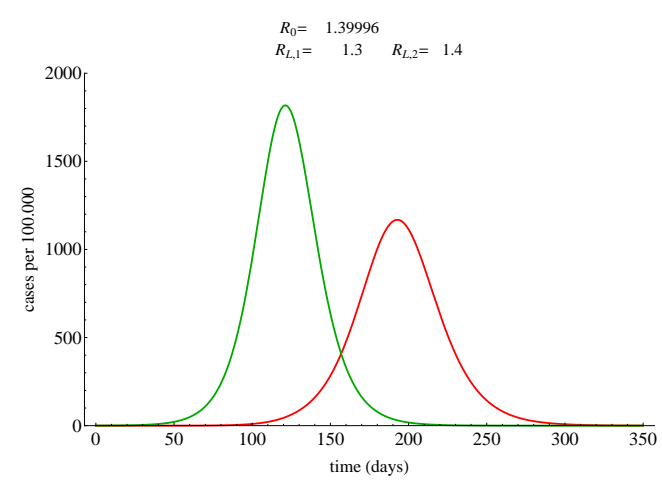

(a) Peak time in Canada: day 195, peak time in Mexico: day 123 when $\gamma_{1}=\frac{1}{15}, \gamma_{2}=\frac{1}{15}$.

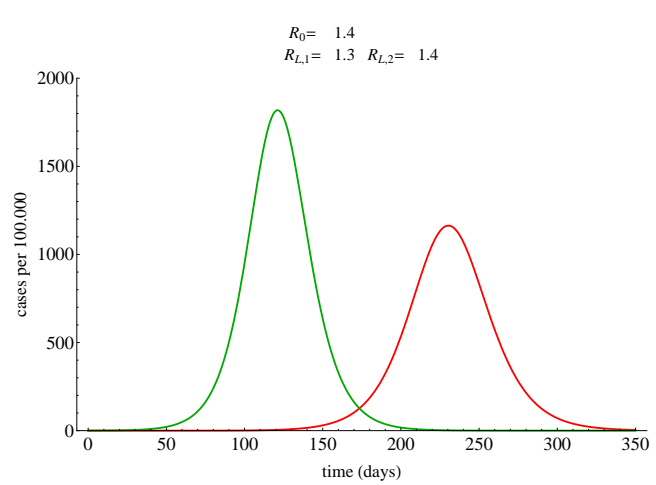

(b) Peak time in Canada: day 230, peak time in Mexico: day 123 when $\gamma_{1}=\frac{1}{0.2}, \gamma_{2}=\frac{1}{0.2}$.

Figure 3.11: In case of a single initial outbreak in Mexico (region 2, green), the outbreak in Canada (region 1, red) can be delayed by 35 days if the average stay of visitors is reduced from 15 days to five hours. For the simulations, we set $\mathcal{R}_{L, 1}=1.3, \mathcal{R}_{L, 2}=1.4, \tau=0.25$, $\beta^{T}=15, \mu_{E}^{-1}=1.4, \mu_{I}^{-1}=3, \mu_{A}^{-1}=4.1, p=0.6, \rho=0.1$.

sort cities like Cancun (177000) and Puerto Vallarta (105000) between January and April, as Canadians return home from winter vacations. However, Mexico City is a steady source of inflowing air traffic throughout the year (190000). This asymmetric travel behavior may cause very dissimilar epidemic courses if we consider outbreaks in different parts of the year.

In order to incorporate the phenomenon of this asymmetric travel behavior of passengers traveling between Canada and Mexico, we divide the course of the year into two phases. Between January and April (Phase I) the daily volume of Canadian residents traveling to Mexico is significantly higher than during the other 8 months of the year (May-December, Phase II). We denote the travel rates of Canadian residents toward Mexico in Phase I and Phase II by $\alpha_{1}^{I}$ and $\alpha_{1}^{I I}$, respectively. Parameter $\Omega_{1}$ was introduced in Section 3.5 to denote the annual volume of Canadians entering Mexico, and using the data of Table 3.4, we obtain that $\Omega_{1}=655000 \frac{15}{15+2}$. We define $\alpha_{1}(t)$, the traveling rate of Canadian residents toward Mexico at time $t$ as follows: we assume that the passenger traffic from Canada to resort cities like Cancun and Puerto Vallarta is due to Canadian residents only, who travel to Mexico for vacation, and all travelers to these cities arrive to Mexico during the first four months of the year. We denote the number of all residents of region 1 (Canada) at time $t$ by $N_{1}^{r}(t)$. We get that

$$
\alpha_{1}(t)=\alpha_{1}^{I I}=\frac{\Omega_{1}-177000-105000}{365} \frac{1}{N_{1}^{r}(t)}
$$




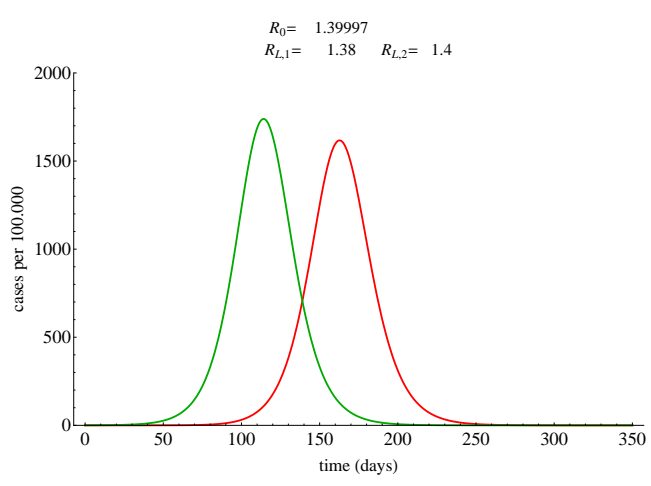

Figure 3.12: Epidemic curves of Canada (region 1, red, peak time: day 160) and Mexico (region 2, green, peak time: day 117) when peak times were fitted to the real morbidity data of the first wave of the 2009 H1N1 and day 0 corresponds to December the 31st 2008. Travel rates arise from [24], historical peak times (day 117-123 in Mexico, day 155-162 in Canada) were derived from $[32,35,52]$. We set $\mathcal{R}_{L, 1}=1.38, \mathcal{R}_{L, 2}=1.4, \tau=0.25$, $\gamma_{1}^{-1}=\gamma_{2}^{-1}=15, \beta^{T}=20, \mu_{E}^{-1}=1.4, \mu_{I}^{-1}=2.7, \mu_{A}^{-1}=4.1, p=0.6, \rho=0.1$.

for $t>121$, i.e., in Phase II and

$$
\alpha_{1}(t)=\alpha_{1}^{I}=\alpha_{1}^{I I}+\frac{177000+105000}{121} \frac{1}{N_{1}^{r}(t)}
$$

for $0 \leq t \leq 121$, where $t=0$ corresponds to December 31, 2008 .

As mentioned above, a significant part of the passenger traffic between Canada and Mexico is due to Canadian vacationers who visit holiday resorts in the first four months of the year. Although the news about an epidemic outbreak might not make people cancel their vacation, it may affect the length of their stay in the affected area. We considered two scenarios for the average length of visitors' stay to reveal the importance of this time period. Figure 3.11 shows that the smaller the value of parameters $\frac{1}{\gamma_{1}}$ and $\frac{1}{\gamma_{2}}$ is, the later the pandemic hits Canada (red curve) if we assume that the first cases were identified in Mexico (green curve). If visitors spend 15 days on average in the other region, the pandemic peaks 35 days earlier in Canada than if we consider an 0.2 day-long (approximately five hours, usual waiting time of transit passengers at airports) stay only. These results were obtained using parameter values $\tau=0.25, \mathcal{R}_{L, 1}=1.3, \mathcal{R}_{L, 2}=1.4$ and the population size of Mexico was set 112.323 million.

\subsubsection{Fitting the model to the $2009 \mathrm{~A}(\mathrm{H} 1 \mathrm{~N} 1)$ pandemic}

To illustrate the applicability of our approach, we fitted the model to the first wave of the 2009 A(H1N1)v pandemic in Canada and Mexico. For the simulation, we chose reasonable values for epidemiological parameters from the ranges of Table 3.3, moreover we used real 
demographic and air traffic data of the Canada-Mexico origin-destination pair. Travel rates were derived from [24], and we set $\tau=0.25$. According to the public reports of the Mexican Social Security Institute [32], WHO Global Influenza Virological Surveillance [52] and the Public Health Agency of Canada [35], the epidemic peaked around week 18 in Mexico and weeks 23-24 in Canada. If day 0 corresponds to December the 31st 2008, then historical peak times are obtained around days 117-123 in Mexico and around days 155-162 in Canada. For the simulations, we estimated the local reproduction numbers to ensure that the peak times of the epidemic curves fit the real morbidity data. The result can be seen in Figure 3.12, where $\mathcal{R}_{L, 1}=1.38$ and $\mathcal{R}_{L, 2}=1.4$. These local reproduction numbers match the results of $[4,46,48]$.

We performed a systematic analysis to reveal the sensitivity to several key parameters. The analysis showed the robustness of the presented fitting in parameters $\gamma_{1}, \gamma_{2}, p$ and $\rho$, although it turned out that the length of latency and infectious periods, the transmission rate during travel and the local reproduction numbers strongly affect the peak times. We wish to emphasize the utmost importance of incorporating disease dynamics during transportation into our model. The discussion around Figures 3.8(c) and 3.9 in Section 3.6 clearly shows that ignoring the possibility of on-board disease transmission results in delayed peak times, thus we could not have had a fitting as presented in Figure 3.12 if we had neglected travel-related infections while keeping every other parameter fixed. To ensure that the curve of Canada peaks around day 155-162 in the absence of travel infections, a much higher value for the Canadian reproduction number $\mathcal{R}_{L, 1}$ would be necessary, which is unrealistic according to the above mentioned references.

\subsection{Conclusion}

Recent epidemics like the 2002-2003 SARS outbreak and the 2009 pandemic influenza A(H1N1) exemplified the role of the global air transportation network played in the worldwide spread of infectious diseases. The topic of epidemic spread due to human transportation has recently been examined in several studies. The metapopulation models in $[2,3,36,50]$ describe the spatial dispersal of infected individuals in connected regions, although ignore the fact that long distance travel such as intercontinental flights provides a platform for on-board transmission of the disease ([16, 49]). The studies [10, 28, 33, 34, 42] account for the fact that, since the progress of the above mentioned diseases is fast, the time needed for transportation between regions is not negligible. They consider the possibility of disease transmission during travel, although the standard SIS-type models used in these works might not be suitable for modeling influenza or SARS. 
We introduced a dynamic model which describes the spread of an infectious disease in and between two regions which are connected by transportation. In the model setup we distinguished local residents from temporary visitors because they might have very different contact rates, mixing patterns and travel behavior. We used the SEAIR model as a basic epidemic building block in the regions and also during the travel, and we modeled disease dynamics during travel by a system structured by travel time. We showed that our model is equivalent to a system of nonlinear functional differential equations with dynamically defined delayed feedback, and we examined the fundamental dynamic properties of the system.

We detailed the computation of the basic reproduction number, which is a threshold quantity for epidemic outbreaks, and discussed the dependence of $\mathcal{R}_{0}$ on several key model parameters. The analysis demonstrates the importance of incorporating the phenomenon of disease transmission during transportation: transmission rates during travel can be much higher than under usual circumstances and our results show that $\beta^{T}$ may significantly alter the value of $\mathcal{R}_{0}$. We parametrized our model for influenza and performed simulations with real demographic and air traffic data. Three origin-destination pairs were introduced for the regions to demonstrate the effect of changing the value of various key model parameters and addressing possible interventions. We showed the applicability of our approach by fitting the model to the first wave of the $2009 \mathrm{~A}(\mathrm{H} 1 \mathrm{~N} 1)$ influenza pandemic in Canada and Mexico.

Our results, in conjunction with recent studies [16, 28, 49], support that considering disease transmission during travel is of particular interest to model the spread of diseases with fast progression. We demonstrated that simpler models which ignore on-board infections can seriously overestimate the time a region has before the epidemic wave arrives. 


\section{Chapter 4}

\section{Epidemic models with travel-related infection}

In the introduction we presented a class of models for the spread of infectious diseases in regions which are connected by means of long distance travel. The SEAIR-based model we established and analyzed in Chapter 3 describes how the global airline network contributes to the propagation of pandemics of the present age, like SARS or influenza. Our results highlight the importance of including travel time and disease dynamics during travel in the model: the invasion of disease free regions is highly expedited by the elevated transmission potential during transportation. Intervention techniques like partial or full airport closure are considered to be potential tools in epidemic prevention and control, as these strategies are aimed to prevent a situation of multiple induced outbreaks generated by infected agents who arrive from endemic regions. In this chapter, we further investigate the topic of infection spread on travel networks as we consider a simple disease transmission model in two connected regions with an entry screening procedure initiated for travelers upon arrival to a region. We also formulate an epidemic model that describes the propagation of a disease in a population of individuals who are distributed over an arbitrary $r$ number of regions, which are connected by a general transportation network. We will see that the model setup leads to a system of autonomous equations with multiple delays, thus we extend our framework elaborated in Chapter 2 to such systems. Instead of the typical assumption of modeling disease transmission by standard incidence, we consider a general infection term and give condition for the existence of solutions in the model.

\subsection{An epidemic model with entry screening}

We formulate a dynamic model describing the spread of an infectious disease in two regions, and also during travel from one region to the other. We assume that the time required to 


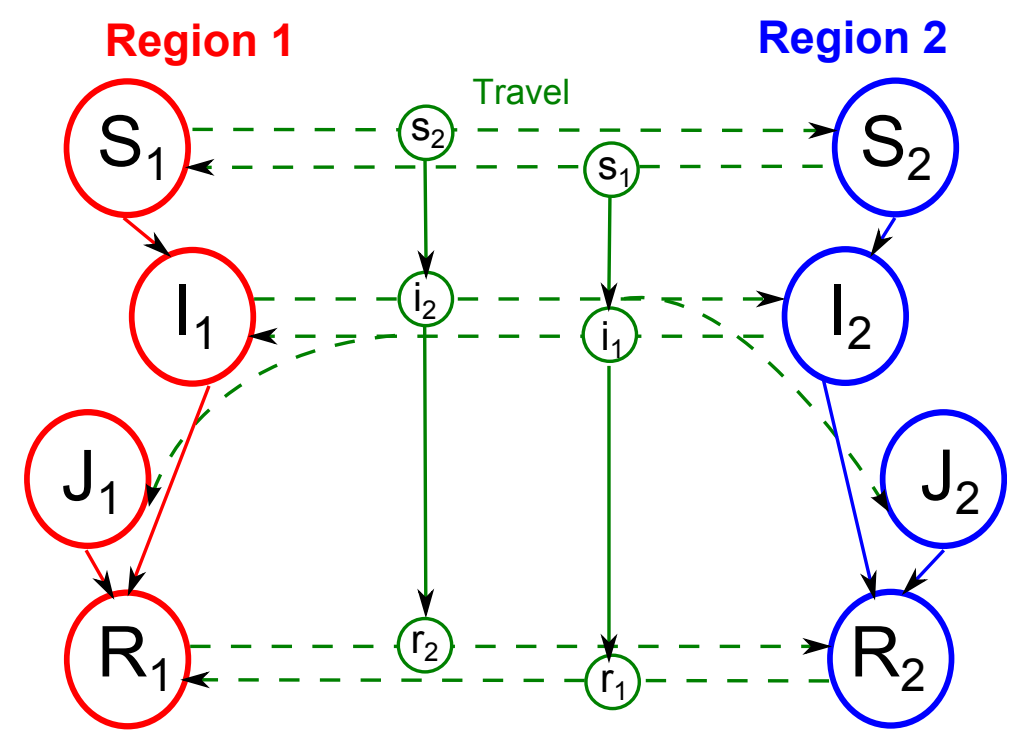

Figure 4.1: Color-coded flow chart of disease transmission and travel dynamics of the SIRJ model. The disease transmission in the two regions is shown in two different columns, the disease progresses vertically from the top to the bottom (solid arrows). Green dashed arrows represent traveling. Green solid arrows show the dynamics of the pandemic during the course of the travel. The description of the variables can be found in Table 4.1.

complete travel between the regions is not negligible. We divide the entire populations of the two regions into the disjoint classes $S_{1}, I_{1}, R_{1}, J_{1}, S_{2}, I_{2}, R_{2}$ and $J_{2}$. Lower index denotes the current region, letters $S$ and $R$ represent the compartments of susceptible and recovered individuals, respectively. We assume that individuals are traveling between the regions and travelers are requested to undergo an entry screening procedure before entering a region after travel. The purpose of the examination is to detect travelers who are infected with the disease and isolate them in order to minimize the chances of an infected agent spreading the infection in a disease free region. Such interventions were proven to have significant effect in mitigating the severity of epidemic outbreaks. Some individuals who are infected with the disease get screened out upon arrival to a region, so they become isolated and belong to class $J$. Others whose illness remains hidden by the examination, and those who are sick but do not travel are in class $I$ and we simply call them infecteds.

Let $S_{1}(t), I_{1}(t), R_{1}(t), J_{1}(t), S_{2}(t), I_{2}(t), R_{2}(t)$ and $J_{2}(t)$ be the number of individuals belonging to $S_{1}, I_{1}, R_{1}, J_{1}, S_{2}, I_{2}, R_{2}$ and $J_{2}$, respectively, at time $t$. Susceptible, infected and recovered individuals of region 1 travel to region 2 by travel rate $\alpha_{1}$. The travel rate of individuals in classes $S_{2}, I_{2}$ and $R_{2}$ from region 2 to region 1 is denoted by $\alpha_{2}$. Isolated individuals are not allowed to travel, moreover we assume that they do not make contact with individuals in other classes until they recover. Model parameters $\mu_{1}$ and $\mu_{2}$ represent 


\begin{tabular}{|ll|}
\hline & \multicolumn{1}{c|}{ Variables and key model parameters } \\
\hline$S_{1}, I_{1}, R_{1}, J_{1}$ & Susceptible, infected, recovered and isolated individuals in region 1 \\
$S_{2}, I_{2}, R_{2}, J_{2}$ & Susceptible, infected, recovered and isolated individuals in region 2 \\
$s_{1}, i_{1}, r_{1}$ & Density of susceptible, infected and recovered individuals \\
& during the travel from region 2 to region 1 \\
$s_{2}, i_{2}, r_{2}$ & Density of susceptible, infected and recovered individuals \\
& during the travel from region 1 to region 2 \\
$\beta_{1}, \beta_{2}$ & Transmission rate in region 1 and in region 2 \\
$\alpha_{1}, \alpha_{2}$ & Traveling rate of individuals in region 1 and in region 2 \\
$\mu_{1}, \mu_{2}$ & Recovery rate of infected and isolated individuals \\
$p_{1}, p_{2}$ & in region 1 and in region 2 \\
& Probability of screening out infected travelers \\
$\tau$ & arriving to region 1 and to region 2 \\
$\beta^{T}$ & Duration of travel between the regions \\
$\mu^{T}$ & Transmission rate during travel \\
\hline
\end{tabular}

Table 4.1: Variables and parameters of the SIRJ model. In the table, "density" means the density with respect to the time elapsed since the start of travel.

the recovery rate of infected and isolated individuals in region 1 and region 2, we denote the transmission rates in region 1 and region 2 by $\beta_{1}$ and $\beta_{2}$.

Let $s_{1}, i_{1}, r_{1}$ and $s_{2}, i_{2}, r_{2}$ denote the classes of susceptible, infected, recovered individuals during the trip to region 1 and to region 2, respectively. The recovery rate of infecteds in travel is $\mu^{T}$, they transmit the disease by rate $\beta^{T}$ during the course of travel. Let $\tau>0$ denote the time required to complete a one-way trip, which is assumed to be fixed. To describe the disease dynamics during the travel, for each $t_{*}$ we define $s_{1}\left(\theta ; t_{*}\right), i_{1}\left(\theta ; t_{*}\right)$, $r_{1}\left(\theta ; t_{*}\right), s_{2}\left(\theta ; t_{*}\right), i_{2}\left(\theta ; t_{*}\right)$ and $r_{2}\left(\theta ; t_{*}\right)$ as the density of individuals with respect to $\theta$ who started travel at time $t_{*}$ and belong to classes $s_{1}, i_{1}, r_{1}, s_{2}, i_{2}$ and $r_{2}$, respectively, where $\theta \in[0, \tau]$ denotes the time elapsed since the beginning of the trip. The total density of individuals who started travel at $t_{*}$ is constant during the trip, that is,

$$
\begin{aligned}
& s_{1}\left(\theta ; t_{*}\right)+i_{1}\left(\theta ; t_{*}\right)+r_{1}\left(\theta ; t_{*}\right)=s_{1}\left(0 ; t_{*}\right)+i_{1}\left(0 ; t_{*}\right)+r_{1}\left(0 ; t_{*}\right), \\
& s_{2}\left(\theta ; t_{*}\right)+i_{2}\left(\theta ; t_{*}\right)+r_{2}\left(\theta ; t_{*}\right)=s_{2}\left(0 ; t_{*}\right)+i_{2}\left(0 ; t_{*}\right)+r_{2}\left(0 ; t_{*}\right)
\end{aligned}
$$

for all $\theta \in[0, \tau]$. By choosing $\theta=\tau, t_{*}=t-\tau$, the terms $s_{1}(\tau ; t-\tau), r_{1}(\tau ; t-\tau)$ and $s_{2}(\tau ; t-\tau), r_{2}(\tau ; t-\tau)$ express the inflow of susceptible and recovered individuals arriving to region 1 to compartments $S_{1}, R_{1}$, and to region 2 to compartments $S_{2}, R_{2}$, 
respectively, at time $t$. We assume that travelers undergo an examination upon the arrival to region 1 and 2 , which detects infection by infecteds with probability $0<p_{1}, p_{2}<1$. This implies that the densities $p_{1} i_{1}(\tau ; t-\tau)$ and $p_{2} i_{2}(\tau ; t-\tau)$ determine individuals who enter $J_{1}$ and $J_{2}$, respectively, at time $t$, since $p_{1}$ and $p_{2}$ are the probabilities that infected travelers get screened out upon arrival. However, infected individuals enter classes $I_{1}$ and $I_{2}$ with probabilities $1-p_{1}$ and $1-p_{2}$ upon completing a trip, hence $\left(1-p_{1}\right) i_{1}(\tau ; t-\tau)$ and $\left(1-p_{2}\right) i_{2}(\tau ; t-\tau)$ give the inflow to classes $I_{1}$ and $I_{2}$, respectively, at time $t$.

The flow chart of the model is depicted in Figure 4.1, see Table 4.1 for variables and key model parameters. We obtain the following system of differential equations for the disease spread in the regions, where disease transmission is modeled by standard incidence:

$$
\left\{\begin{array}{l}
\dot{S}_{1}(t)=-\beta_{1} \frac{S_{1}(t) I_{1}(t)}{S_{1}(t)+I_{1}(t)+R_{1}(t)}-\alpha_{1} S_{1}(t)+s_{1}(\tau ; t-\tau), \\
\dot{I}_{1}(t)=\beta_{1} \frac{S_{1}(t) I_{1}(t)}{S_{1}(t)+I_{1}(t)+R_{1}(t)}-\mu_{1} I_{1}(t)-\alpha_{1} I_{1}(t)+\left(1-p_{1}\right) i_{1}(\tau ; t-\tau), \\
\dot{R}_{1}(t)=\mu_{1}\left(I_{1}(t)+J_{1}(t)\right)-\alpha_{1} R_{1}(t)+r_{1}(\tau ; t-\tau), \\
\dot{J}_{1}(t)=-\mu_{1} J_{1}(t)+p_{1} i_{1}(\tau ; t-\tau) \\
\dot{S}_{2}(t)=-\beta_{2} \frac{S_{2}(t) I_{2}(t)}{S_{2}(t)+I_{2}(t)+R_{2}(t)}-\alpha_{2} S_{2}(t)+s_{2}(\tau ; t-\tau), \\
\dot{I}_{2}(t)=\beta_{2} \frac{S_{2}(t) I_{2}(t)}{S_{2}(t)+I_{2}(t)+R_{2}(t)}-\mu_{2} I_{2}(t)-\alpha_{2} I_{2}(t)+\left(1-p_{2}\right) i_{2}(\tau ; t-\tau), \\
\dot{R}_{2}(t)=\mu_{2}\left(I_{2}(t)+J_{2}(t)\right)-\alpha_{2} R_{2}(t)+r_{2}(\tau ; t-\tau) \\
\dot{J}_{2}(t)=-\mu_{2} J_{2}(t)+p_{2} i_{2}(\tau ; t-\tau)
\end{array}\right.
$$

For each $t_{*}$, the following system describes the evolution of the densities during the travel which started at time $t_{*}$ :

$$
\left\{\begin{array}{l}
\frac{\mathrm{d}}{\mathrm{d} \theta} s_{1}\left(\theta ; t_{*}\right)=-\beta^{T} \frac{s_{1}\left(\theta ; t_{*}\right) i_{1}\left(\theta ; t_{*}\right)}{s_{1}\left(\theta ; t_{*}\right)+i_{1}\left(\theta ; t_{*}\right)+r_{1}\left(\theta ; t_{*}\right)} \\
\frac{\mathrm{d}}{\mathrm{d} \theta} i_{1}\left(\theta ; t_{*}\right)=\beta^{T} \frac{s_{1}\left(\theta ; t_{*}\right) i_{1}\left(\theta ; t_{*}\right)}{s_{1}\left(\theta ; t_{*}\right)+i_{1}\left(\theta ; t_{*}\right)+r_{1}\left(\theta ; t_{*}\right)}-\mu^{T} i_{1}\left(\theta ; t_{*}\right) \\
\frac{\mathrm{d}}{\mathrm{d} \theta} r_{1}\left(\theta ; t_{*}\right)=\mu^{T} i_{1}\left(\theta ; t_{*}\right) \\
\frac{\mathrm{d}}{\mathrm{d} \theta} s_{2}\left(\theta ; t_{*}\right)=-\beta^{T} \frac{s_{2}\left(\theta ; t_{*}\right) i_{2}\left(\theta ; t_{*}\right)}{s_{2}\left(\theta ; t_{*}\right)+i_{2}\left(\theta ; t_{*}\right)+r_{2}\left(\theta ; t_{*}\right)} \\
\frac{\mathrm{d}}{\mathrm{d} \theta} i_{2}\left(\theta ; t_{*}\right)=\beta^{T} \frac{s_{2}\left(\theta ; t_{*}\right) i_{2}\left(\theta ; t_{*}\right)}{s_{2}\left(\theta ; t_{*}\right)+i_{2}\left(\theta ; t_{*}\right)+r_{2}\left(\theta ; t_{*}\right)}-\mu^{T} i_{2}\left(\theta ; t_{*}\right) \\
\frac{\mathrm{d}}{\mathrm{d} \theta} r_{2}\left(\theta ; t_{*}\right)=\mu^{T} i_{2}\left(\theta ; t_{*}\right)
\end{array}\right.
$$

where again we assume standard incidence for the disease transmission. Note that the dimensions of systems (4.1) and (4.2) are different. 
For $\theta=0$, the densities are determined by the rates individuals start their travels from one region to the other at time $t_{*}$. Hence, the initial values for system (4.2) at $\theta=0$ are given by

$$
\begin{cases}s_{1}\left(0 ; t_{*}\right)=\alpha_{2} S_{2}\left(t_{*}\right), & s_{2}\left(0 ; t_{*}\right)=\alpha_{1} S_{1}\left(t_{*}\right), \\ i_{1}\left(0 ; t_{*}\right)=\alpha_{2} I_{2}\left(t_{*}\right), & i_{2}\left(0 ; t_{*}\right)=\alpha_{1} I_{1}\left(t_{*}\right), \\ r_{1}\left(0 ; t_{*}\right)=\alpha_{2} R_{2}\left(t_{*}\right), & r_{2}\left(0 ; t_{*}\right)=\alpha_{1} R_{1}\left(t_{*}\right) .\end{cases}
$$

Now we turn our attention to the terms $s_{1}(\tau ; t-\tau),\left(1-p_{1}\right) i_{1}(\tau ; t-\tau), r_{1}(\tau ; t-\tau)$, $p_{1} i_{1}(\tau ; t-\tau), s_{2}(\tau ; t-\tau),\left(1-p_{2}\right) i_{2}(\tau ; t-\tau), r_{2}(\tau ; t-\tau)$ and $p_{2} i_{2}(\tau ; t-\tau)$ in system (4.1), which are the densities of individuals arriving to classes $S_{1}, I_{1}, R_{1}, J_{1}, S_{2}, I_{2}, R_{2}$ and $J_{2}$, respectively, at time $t$ upon completing a one-way trip. At time $t$, these terms are determined by the solution of system (4.2) with initial values (4.3) for $t_{*}=t-\tau$ at $\theta=\tau$. An individual may move to a different compartment during travel, for example a susceptible individual who travels from region 1 may arrive as infected to region 2 , as given by the dynamics of system (4.2).

Next we specify initial values for system (4.1) at $t=0$. Since a one-way trip takes $\tau$ units of time to complete, arrivals to region 1 are determined by the state of classes $S_{2}, I_{2}$ and $R_{2}$ at $t-\tau$ and vice versa, via the solution of systems (4.2) and (4.3). Thus, we set up the initial functions as follows:

$$
\left\{\begin{aligned}
S_{1}(u) & =\varphi_{S, 1}(u), & S_{2}(u) & =\varphi_{S, 2}(u) \\
I_{1}(u) & =\varphi_{I, 1}(u), & I_{2}(u) & =\varphi_{I, 2}(u) \\
R_{1}(u) & =\varphi_{R, 1}(u), & R_{2}(u) & =\varphi_{R, 2}(u) \\
J_{1}(u) & =\varphi_{J, 1}(u), & J_{2}(u) & =\varphi_{J, 2}(u)
\end{aligned}\right.
$$

where $u \in[-\tau, 0]$ and $\varphi_{K, j}$ is continuous for each $j \in\{1,2\}, K \in\{S, I, R, J\}$.

\subsubsection{The compact form of the model}

For a given $t_{*}$, we define $y(\theta)=\left(s_{1}\left(\theta ; t_{*}\right), i_{1}\left(\theta ; t_{*}\right), r_{1}\left(\theta ; t_{*}\right), s_{2}\left(\theta ; t_{*}\right), i_{2}\left(\theta ; t_{*}\right), r_{2}\left(\theta ; t_{*}\right)\right)^{T}$ and let $g=\left(g_{1}, g_{2}, g_{3}, g_{4}, g_{5}, g_{6}\right)^{T}$, where $y:[0, \tau] \rightarrow \mathbb{R}^{6}$ and $g: \mathbb{R}^{6} \rightarrow \mathbb{R}^{6}$. Function $g$ is defined as $g_{j}(y)$ equals the right-hand side of the equation for $y_{j}$ in system (4.2) for $j=1, \ldots, 6$. For instance,

$$
g_{5}(y)=\beta^{T} \frac{y_{4} y_{5}}{y_{4}+y_{5}+y_{6}}-\mu^{T} y_{5} .
$$

Then we find that for each fixed $t_{*},(2.10)$ is a compact form of (4.2) for $m=6$, with the initial value $y_{*}$ set as (4.3). To apply the results we have obtained in Chapter 2 for the general system (2.10) it is necessary to prove that the conditions made in Section 2.3 
hold for the SIRJ model. We have seen that the benefit of showing the global Lipschitz property (gLip) for $g$ is twofold, since it guarantees the existence of the solution of (2.10) on $[0, \infty$ ), and trivially also yields that (Lip) holds for $g$. First we need a simple result on the nonnegativity of solutions of (4.2), which is stated here without proof since it is immediate from the equations. We remark, however, that existence of the solution follows from the continuity of $g$, without assuming the Lipschitz property ([22]).

Proposition 4.1. For each fixed $t_{*}$, the solution of system (4.2) is nonnegative for nonnegative initial values, where it exists.

Proposition 4.2. Function g, as defined for the SIRJ model, satisfies the global Lipschitz condition (gLip) on each bounded subset of $\mathbb{R}_{+}^{6}$. It follows that for each fixed $t_{*}$, there exists a unique nonnegative solution of system (4.2) on $[0, \infty)$ for nonnegative initial data.

Proof. The function $g: \mathbb{R}^{6} \rightarrow \mathbb{R}^{6}$ possesses the global Lipschitz condition ( $g$ Lip) if there exists a Lipschitz constant $K_{g}>0$ such that $|g(z)-g(w)|_{6} \leq K_{g}|z-w|_{6}$ holds for any $z, w \in \mathbb{R}_{+}^{6}, z \neq 0, w \neq 0$. First, we show that there exists a $\left(K_{g}\right)_{1}>0$ such that $\left|g_{1}(z)-g_{1}(w)\right| \leq\left(K_{g}\right)_{1}|z-w|_{6}$ (all norms will be denoted by $|\cdot|$ throughout the proof).

For $z, w \in \mathbb{R}_{+}^{6}, z \neq 0, w \neq 0$, it holds that

$$
\begin{aligned}
\left|g_{1}(z)-g_{1}(w)\right|= & -\beta^{T} \frac{z_{1} z_{2}}{z_{1}+z_{2}+z_{3}}+\beta^{T} \frac{w_{1} w_{2}}{w_{1}+w_{2}+w_{3}} \mid \\
\leq & \beta^{T}\left|\frac{w_{1} w_{2}}{w_{1}+w_{2}+w_{3}}-\frac{z_{1} z_{2}}{z_{1}+z_{2}+z_{3}}\right| \\
= & \beta^{T} \mid \frac{w_{1} w_{2}}{w_{1}+w_{2}+w_{3}}-\frac{w_{1} z_{2}}{w_{1}+w_{2}+w_{3}} \\
& +\frac{w_{1} z_{2}}{w_{1}+w_{2}+w_{3}}-\frac{w_{1} z_{2}}{w_{1}+z_{2}+w_{3}}+\frac{w_{1} z_{2}}{w_{1}+z_{2}+w_{3}}-\frac{w_{1} z_{2}}{w_{1}+z_{2}+z_{3}} \\
& +\frac{w_{1} z_{2}}{w_{1}+z_{2}+z_{3}}-\frac{w_{1} z_{2}}{z_{1}+z_{2}+z_{3}}+\frac{w_{1} z_{2}}{z_{1}+z_{2}+z_{3}}-\frac{z_{1} z_{2}}{z_{1}+z_{2}+z_{3}} \mid \\
\leq & \beta^{T}\left(\left|\frac{w_{1} w_{2}}{w_{1}+w_{2}+w_{3}}-\frac{w_{1} z_{2}}{w_{1}+w_{2}+w_{3}}\right|\right. \\
& +\left|\frac{w_{1} z_{2}}{w_{1}+w_{2}+w_{3}}-\frac{w_{1} z_{2}}{w_{1}+z_{2}+w_{3}}\right|+\left|\frac{w_{1} z_{2}}{w_{1}+z_{2}+w_{3}}-\frac{w_{1} z_{2}}{w_{1}+z_{2}+z_{3}}\right| \\
& \left.+\left|\frac{w_{1} z_{2}}{w_{1}+z_{2}+z_{3}}-\frac{w_{1} z_{2}}{z_{1}+z_{2}+z_{3}}\right|+\left|\frac{w_{1} z_{2}}{z_{1}+z_{2}+z_{3}}-\frac{z_{1} z_{2}}{z_{1}+z_{2}+z_{3}}\right|\right)
\end{aligned}
$$




$$
\begin{aligned}
= & \beta^{T}\left(\left|w_{2}-z_{2}\right|\left|\frac{w_{1}}{w_{1}+w_{2}+w_{3}}\right|+\left|z_{2}-w_{2}\right|\left|\frac{w_{1} z_{2}}{\left(w_{1}+w_{2}+w_{3}\right)\left(w_{1}+z_{2}+w_{3}\right)}\right|\right. \\
& +\left|z_{3}-w_{3}\right|\left|\frac{w_{1} z_{2}}{\left(w_{1}+z_{2}+w_{3}\right)\left(w_{1}+z_{2}+z_{3}\right)}\right| \\
& \left.+\left|z_{1}-w_{1}\right|\left|\frac{w_{1} z_{2}}{\left(w_{1}+z_{2}+z_{3}\right)\left(z_{1}+z_{2}+z_{3}\right)}\right|+\left|w_{1}-z_{1}\right|\left|\frac{z_{2}}{z_{1}+z_{2}+z_{3}}\right|\right) \\
\leq & \beta^{T}\left(2\left|w_{2}-z_{2}\right|+\left|z_{3}-w_{3}\right|+2\left|z_{1}-w_{1}\right|\right) \\
\leq & 5 \beta^{T}|w-z|,
\end{aligned}
$$

where we used that the inequality $\frac{a}{a+b+c} \leq 1$ holds for any $(a, b, c) \in \mathbb{R}_{+}^{3} \backslash\{0\}$. We define $\left(K_{g}\right)_{1}=5 \beta^{T}$, and note that the same constant works for $\left(K_{g}\right)_{4}$ in $\left|g_{4}(z)-g_{4}(w)\right| \leq$ $\left(K_{g}\right)_{4}|z-w|$ as

$$
\begin{aligned}
\left|g_{4}(z)-g_{4}(w)\right| & =\left|-\beta^{T} \frac{z_{5} z_{6}}{z_{5}+z_{6}+z_{7}}+\beta^{T} \frac{w_{5} w_{6}}{w_{5}+w_{6}+w_{7}}\right| \\
& \leq\left(K_{g}\right)_{4}|w-z| .
\end{aligned}
$$

Furthermore, we derive

$$
\begin{aligned}
\left|g_{2}(z)-g_{2}(w)\right| & =\left|\beta^{T} \frac{z_{1} z_{2}}{z_{1}+z_{2}+z_{3}}-\mu^{T} z_{2}-\beta^{T} \frac{w_{1} w_{2}}{w_{1}+w_{2}+w_{3}}+\mu^{T} w_{2}\right| \\
& \leq \mu^{T}\left|w_{2}-z_{2}\right|+\beta^{T}\left(2\left|w_{2}-z_{2}\right|+\left|z_{3}-w_{3}\right|+2\left|w_{1}-z_{1}\right|\right) \\
& \leq\left(\mu^{T}+5 \beta^{T}\right)|w-z|
\end{aligned}
$$

where $\mu^{T}+5 \beta^{T}$ is a suitable choice for $\left(K_{g}\right)_{2}$, and it is clear that $\left(K_{g}\right)_{5}=\mu^{T}+5 \beta^{T}$ satisfies

$$
\begin{aligned}
\left|g_{5}(z)-g_{5}(w)\right| & =\left|\beta^{T} \frac{z_{4} z_{5}}{z_{4}+z_{5}+z_{6}}-\mu^{T} z_{5}-\beta^{T} \frac{w_{4} w_{5}}{w_{4}+w_{5}+w_{6}}+\mu^{T} w_{5}\right| \\
& \leq\left(K_{g}\right)_{5}|w-z| .
\end{aligned}
$$

Last, by the computations

$$
\begin{aligned}
\left|f_{3}(z)-f_{3}(w)\right| & =\left|\mu^{T} z_{2}-\mu^{T} w_{2}\right| \\
& \leq \mu^{T}|w-z|, \\
\left|f_{6}(z)-f_{6}(w)\right| & =\left|\mu^{T} z_{4}-\mu^{T} w_{4}\right| \\
& \leq \mu^{T}|w-z|,
\end{aligned}
$$

we arrive to $\left(K_{g}\right)_{3}=\mu^{T}$ and $\left(K_{g}\right)_{6}=\mu^{T}$. To obtain the global Lipschitz constant for $g$, we simply choose the squared sum of $\left(K_{g}\right)_{1}, \ldots,\left(K_{g}\right)_{6}$. The proof is complete.

Now as the existence and uniqueness of the solutions of (4.2) has been proved on $[0, \tau]$ for each $t_{*}$, we get that the terms $s_{1}(\tau ; t-\tau),\left(1-p_{1}\right) i_{1}(\tau ; t-\tau), r_{1}(\tau ; t-\tau), p_{1} i_{1}(\tau ; t-\tau)$, 
$s_{2}(\tau ; t-\tau),\left(1-p_{2}\right) i_{2}(\tau ; t-\tau), r_{2}(\tau ; t-\tau)$ and $p_{2} i_{2}(\tau ; t-\tau)$, representing inflow by means of travel to the respective compartments in system (4.1), are well-defined. We proceed as we let $x(t)=\left(S_{1}(t), I_{1}(t), R_{1}(t), J_{1}(t), S_{2}(t), I_{2}(t), R_{2}(t), J_{2}(t)\right)^{T}$ for $x:[0, \infty) \rightarrow \mathbb{R}^{8}$ and define $f=\left(f_{1}, f_{2}, f_{3}, f_{4}, f_{5}, f_{6}, f_{7}, f_{8}\right)^{T}, f: \mathbb{R}^{8} \rightarrow \mathbb{R}^{8}$, as for each $j \in\{1, \ldots, 8\} f_{j}(x)$ is the right-hand side of the equation of $x_{j}$ in (4.1) without the inflow from travel; for instance,

$$
f_{1}(x)=-\beta_{1} \frac{x_{1} x_{2}}{x_{1}+x_{2}+x_{3}}-\alpha_{1} x_{1} .
$$

If we let

$$
\begin{cases}h_{1}(v)=\alpha_{2} v_{5}, & h_{4}(v)=\alpha_{1} v_{1}, \\ h_{2}(v)=\alpha_{2} v_{6}, & h_{5}(v)=\alpha_{1} v_{2}, \\ h_{3}(v)=\alpha_{2} v_{7}, & h_{6}(v)=\alpha_{1} v_{3},\end{cases}
$$

$h=\left(h_{1}, h_{2}, h_{3}, h_{4}, h_{5}, h_{6}\right): \mathbb{R}^{8} \rightarrow \mathbb{R}^{6}$, and

$$
\begin{cases}k_{1}(v)=v_{1}, & k_{5}(v)=v_{4} \\ k_{2}(v)=\left(1-p_{1}\right) v_{2}, & k_{6}(v)=\left(1-p_{2}\right) v_{5} \\ k_{3}(v)=v_{3}, & k_{7}(v)=v_{6} \\ k_{4}(v)=p_{1} v_{2}, & k_{8}(v)=p_{2} v_{5}\end{cases}
$$

$k=\left(k_{1}, k_{2}, k_{3}, k_{4}, k_{5}, k_{6}, k_{7}, k_{8}\right): \mathbb{R}^{6} \rightarrow \mathbb{R}^{8}$, then our system (4.1) with initial conditions (4.4) can be written in a closed form as (2.9) for $n=8$. The feasible phase space is the nonnegative cone $C_{+}$of $C=C\left([-\tau, 0], \mathbb{R}^{8}\right)$, the Banach space of continuous functions from $[-\tau, 0]$ to $\mathbb{R}^{8}$ equipped with the supremum norm.

Our aim is to show that there exists a unique solution of system (4.1), moreover nonnegative initial data give rise to nonnegative solution. As (4.1) is equivalent to system (2.9), we can use the framework established in Chapter 2, which yields that these results can be obtained by checking certain conditions on $f, g, h$ and $k$. Corollary 2.12 is applied to get that solutions of system (4.1) preserve nonnegativity (where they exist), which also ensures that it suffices to show the Lipschitz property for $f$ only for nonnegative vectors.

Proposition 4.3. The solution of system (4.1) is nonnegative for nonnegative initial values, where it exists.

Proposition 4.4. Functions $h$ and $k$, as defined for the SIRJ model, possess the global Lipschitz property (gLip) on each bounded subset of $\mathbb{R}_{+}^{8}$ and $\mathbb{R}_{+}^{6}$, respectively. Function $f$, as defined for the SIRJ model, satisfies the global Lipschitz condition (gLip) on each bounded subset of $\mathbb{R}_{+}^{8}$. 
Proof. It immediately follows from the definitions that $h$ and $k$ possess the global Lipschitz condition (gLip), moreover minor modifications in the computations for the Lipschitz constant of $g$ in the proof of Proposition 4.2 yield that the condition holds for $f$. Indeed, the inequalities $\left|f_{j}(z)-f_{j}(w)\right| \leq\left(K_{f}\right)_{j}|z-w|_{8}, j=1, \ldots, 8$, are satisfied if we let $\left(K_{f}\right)_{1}=5 \beta_{1}+\alpha_{1},\left(K_{f}\right)_{2}=5 \beta_{1}+\alpha_{1}+\mu_{1},\left(K_{f}\right)_{3}=2 \mu_{1}+\alpha_{1},\left(K_{f}\right)_{4}=\mu_{1}$ and $\left(K_{f}\right)_{5}=5 \beta_{2}+\alpha_{2},\left(K_{f}\right)_{6}=5 \beta_{2}+\alpha_{2}+\mu_{2},\left(K_{f}\right)_{7}=2 \mu_{2}+\alpha_{2},\left(K_{f}\right)_{8}=\mu_{2}$. Again, with $K_{f}$ defined as the squared sum of these constants we arrive to $|f(z)-f(w)|_{8} \leq K_{f}|z-w|_{8}$, which proves the statement.

The necessary conditions for Corollary 2.10 are satisfied, thus using Remark 2.11 we claim that there exists a unique solution of $(4.1)$ on $[-\tau, \infty)$ with initial conditions (4.4). We recall that nonnegative initial data give rise to a nonnegative solution of (4.1), which means that $C_{+}$is invariant.

Theorem 4.5. For nonnegative initial values system (4.1) has a unique nonnegative solution, which exists on $[-\tau, \infty)$.

\subsection{Multiregional SIR model with general infection term}

We consider an arbitrary $r$ number of regions which are connected by transportation, and present an SIR-based model which describes the spread of infection in and between the regions. We will learn that the compact form of the corresponding system of functional differential equations is similar, though not identical, to systems (2.9) and (2.10) introduced in Chapter 2. Henceforth, we refer to the general framework where applicable, and prove some analogous results in this chapter if the setting considerably differs from the one established in Chapter 2.

We formulate a dynamical model describing the spread of an infectious disease in $r$ regions and also during travel from one region to another. We divide the entire populations of the $r$ regions into the disjoint classes $S_{j}, I_{j}, R_{j}, j \in\{1, \ldots, r\}$, where $S_{j}(t) I_{j}(t), R_{j}(t)$ denote the number of susceptible, infected and recovered individuals, respectively, at time $t$ in region $j, j \in\{1, \ldots, r\}$. For the total population in region $j$ at time $t$, we use the notation

$$
N_{j}(t)=S_{j}(t)+I_{j}(t)+R_{j}(t) .
$$

The incidence in region $j$ is denoted by $\Lambda_{j}\left(S_{j}(t), I_{j}(t), R_{j}(t)\right)$, model parameter $\mu_{j}$ represents the recovery rate of infected individuals in region $j$. We denote the travel rate from region $j$ to region $k$ by $\alpha_{j, k}$ for $j, k \in\{1, \ldots, r\}, j \neq k$, and we let $\alpha_{j, j}=0$.

Let $s_{k, j}, i_{k, j}, r_{k, j}$ denote susceptible, infected and recovered travelers, respectively, where 


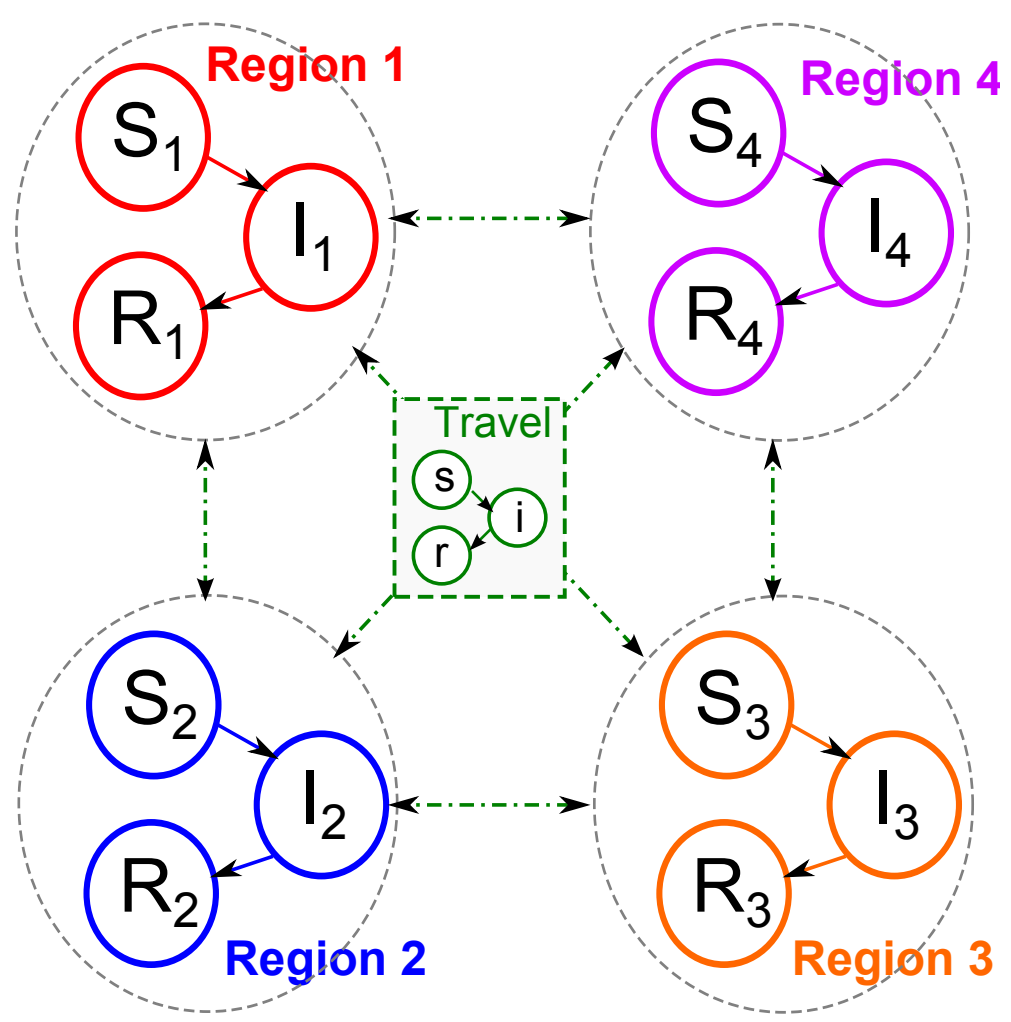

Figure 4.2: Color-coded flow chart of disease transmission and travel dynamics of the multiregional SIR model for $r=4$ regions. The disease transmission in the regions is shown in four blocks, arrows colored with the same colors indicate how the disease progresses. Green dashed-dotted arrows represent traveling. Green solid arrows show the dynamics of the pandemic during the course of the travel. The description of the variables can be found in Table 4.2.

lower index-pair $\{k, j\}, j, k \in\{1, \ldots, r\}, j \neq k$, indicates that individuals are traveling from region $k$ to region $j$. Let $\tau_{k, j}>0$ denote the time required to complete the travel from region $k$ to region $j$, which is assumed to be fixed. To describe the disease dynamics during travel, for each $t_{*}$ we define $s_{k, j}\left(\theta ; t_{*}\right), i_{k, j}\left(\theta ; t_{*}\right), r_{k, j}\left(\theta ; t_{*}\right), j, k \in\{1, \ldots, r\}, j \neq k$, as the density of individuals with respect to $\theta$ who started travel at time $t_{*}$ and belong to class $s_{k, j}, i_{k, j}, r_{k, j}$, respectively, where $\theta \in\left[0, \tau_{k, j}\right]$ denotes the time elapsed since the beginning of the travel. Then $s_{k, j}\left(\tau_{k, j} ; t-\tau_{k, j}\right), i_{k, j}\left(\tau_{k, j} ; t-\tau_{k, j}\right), r_{k, j}\left(\tau_{k, j} ; t-\tau_{k, j}\right)$ express the inflow of individuals arriving from region $k$ to compartments $S_{j}, I_{j}, R_{j}$ at time $t$. Let

$$
n_{k, j}\left(\theta ; t_{*}\right)=s_{k, j}\left(\theta ; t_{*}\right)+i_{k, j}\left(\theta ; t_{*}\right)+r_{k, j}\left(\theta ; t_{*}\right)
$$

denote the total density of individuals with respect to $\theta$ during the travel from region $k$ to $j$, where $j, k \in\{1, \ldots, r\}$ and $j \neq k$. The total density is constant during travel, i.e., $n_{k, j}\left(\theta ; t_{*}\right)=n_{k, j}\left(0, t_{*}\right)$ for all $\theta \in\left[0, \tau_{k, j}\right]$. During the course of travel from region $k$ to $j$, $\lambda_{k, j}^{T}\left(s_{k, j}\left(\theta ; t_{*}\right), i_{k, j}\left(\theta ; t_{*}\right), r_{k, j}\left(\theta ; t_{*}\right)\right)$ describes the incidence, and we let $\mu_{k, j}^{T}$ denote the 


\begin{tabular}{|ll|}
\hline \multicolumn{2}{|c|}{ Variables and key model parameters } \\
\hline$S_{j}, I_{j}, R_{j}, N_{j}$ & Susceptible, infected, recovered and all individuals in region $j$ \\
$s_{k, j}, i_{k, j}, r_{k, j}, n_{k, j}$ & Density of susceptible, infected, recovered and all individuals \\
& during the travel from region $k$ to region $j$ \\
$\Lambda_{j}$ & Incidence in region $j$ \\
$\lambda_{k, j}^{T}$ & Incidence during travel from region $k$ to region $j$ \\
$\mu_{j}$ & Recovery rate of infected individuals in region $j$ \\
$\mu_{k, j}^{T}$ & Recovery rate during travel from region $k$ to region $j$ \\
$\alpha_{j, k}$ & Travel rate from region $j$ to region $k$ \\
$\tau_{k, j}$ & Duration of travel from region $k$ to region $j$ \\
\hline
\end{tabular}

Table 4.2: Variables and parameters of the multiregional SIR model $(j, k \in\{1, \ldots, r\}$, $j \neq k$ ). In the table, "density" means the density with respect to the time elapsed since the start of travel.

recovery rate.

For convenience, we define functions $s_{j, j}, i_{j, j}, r_{j, j}: \mathbb{R}_{+} \times \mathbb{R}_{+} \rightarrow \mathbb{R}$ as $s_{j, j}\left(\theta ; t_{*}\right)=i_{j, j}\left(\theta ; t_{*}\right)=$ $r_{j, j}\left(\theta ; t_{*}\right) \equiv 0$, and constants $\tau_{j, j}=0$ for each $j \in\{1, \ldots, r\}$. All variables and model parameters are listed in Table 4.2, while Figure 4.2 depicts the flow chart of the model. Based on the assumptions formulated above, we obtain the following system of differential equations for the disease transmission in region $j, j \in\{1, \ldots, r\}$ :

$$
\left\{\begin{array}{l}
\dot{S}_{j}(t)=-\Lambda_{j}(\cdot)-\left(\sum_{k=1}^{r} \alpha_{j, k}\right) S_{j}(t)+\sum_{k=1}^{r} s_{k, j}\left(\tau_{k, j} ; t-\tau_{k, j}\right), \\
\dot{I}_{j}(t)=\Lambda_{j}(\cdot)-\left(\sum_{k=1}^{r} \alpha_{j, k}\right) I_{j}(t)-\mu_{j} I_{j}(t)+\sum_{k=1}^{r} i_{k, j}\left(\tau_{k, j} ; t-\tau_{k, j}\right) \\
\dot{R}_{j}(t)=\mu_{j} I_{j}(t)-\left(\sum_{k=1}^{r} \alpha_{j, k}\right) R_{j}(t)+\sum_{k=1}^{r} r_{k, j}\left(\tau_{k, j} ; t-\tau_{k, j}\right) .
\end{array}\right.
$$

For each $j, k \in\{1, \ldots, n\}, j \neq k$, and for each $t_{*}$, the following system $\left(T_{k, j}\right)$ describes the evolution of the densities during the travel from region $k$ to $j$ which started at time $t_{*}$ :

$$
\left\{\begin{array}{l}
\frac{\mathrm{d}}{\mathrm{d} \theta} s_{k, j}\left(\theta ; t_{*}\right)=-\lambda_{k, j}^{T}(\cdot), \\
\frac{\mathrm{d}}{\mathrm{d} \theta} i_{k, j}\left(\theta ; t_{*}\right)=\lambda_{k, j}^{T}(\cdot)-\mu_{k, j}^{T} i_{k, j}\left(\theta ; t_{*}\right), \\
\frac{\mathrm{d}}{\mathrm{d} \theta} r_{k, j}\left(\theta ; t_{*}\right)=\mu_{k, j}^{T} i_{k, j}\left(\theta ; t_{*}\right) .
\end{array}\right.
$$

For sake of simplicity, in systems $\left(L_{j}\right)$ and $\left(T_{k, j}\right)$ we use the notations $\Lambda_{j}(\cdot)$ and $\lambda_{k, j}^{T}(\cdot)$ for the incidences, where these functions are meant to be evaluated at the respective points. For $\theta=0$, the densities $s_{k, j}\left(\theta ; t_{*}\right), i_{k, j}\left(\theta ; t_{*}\right), r_{k, j}\left(\theta ; t_{*}\right)$ are determined by the rates 
individuals start their travels from region $k$ to region $j$ at time $t_{*}$. Hence, the initial values for system $\left(T_{k, j}\right)$ at $\theta=0$ are given by

$$
\left\{\begin{array}{l}
s_{k, j}\left(0 ; t_{*}\right)=\alpha_{k, j} S_{k}\left(t_{*}\right) \\
i_{k, j}\left(0 ; t_{*}\right)=\alpha_{k, j} I_{k}\left(t_{*}\right), \\
r_{k, j}\left(0 ; t_{*}\right)=\alpha_{k, j} R_{k}\left(t_{*}\right) .
\end{array}\right.
$$

Now we turn our attention to the terms $s_{k, j}\left(\tau_{k, j} ; t-\tau_{k, j}\right), i_{k, j}\left(\tau_{k, j} ; t-\tau_{k, j}\right), r_{k, j}\left(\tau_{k, j} ; t-\tau_{k, j}\right)$, $j, k \in\{1, \ldots, r\}, j \neq k$, in system $\left(L_{j}\right)$, which give the inflow of individuals arriving to classes $S_{j}, I_{j}, R_{j}$, respectively, at time $t$ upon completing a trip from region $k$. At time $t$, these terms are determined by the solution of system $\left(T_{k, j}\right)$ at $\theta=\tau_{k, j}$ with initial values $\left(I V T_{k, j}\right)$ for $t_{*}=t-\tau_{k, j}$, since individuals who left region $k$ with rate $\alpha_{k, j}$ at time $t-\tau_{k, j}$ will enter region $j$ at time $t$.

Next we specify initial values for system $\left(L_{j}\right)$ at $t=0$. Since for $k \in\{1, \ldots, r\}, k \neq j$, travel from region $k$ to region $j$ takes $\tau_{k, j}$ units of time to complete, arrivals to region $j$ at time $t$ are determined by the state of the classes of region $k$ at $t-\tau_{k, j}$, via the solution of system $\left(T_{k, j}\right)$ and initial values $\left(I V T_{k, j}\right)$. Thus, we set up initial values as follows:

$$
\left\{\begin{aligned}
S_{j}(u) & =\varphi_{S, j}(u), \\
I_{j}(u) & =\varphi_{I, j}(u), \\
R_{j}(u) & =\varphi_{R, j}(u),
\end{aligned}\right.
$$

where $u \in[-\tau, 0]$ for $\tau:=\max _{j, k \in\{1, \ldots, r\}} \tau_{k, j}$, moreover $\varphi_{S, j}, \varphi_{I, j}$ and $\varphi_{R, j}$ are continuous functions for each $j \in\{1, \ldots, r\}$.

\subsubsection{The compact form of the model}

For each $j, k \in\{1, \ldots, r\}, j \neq k$ and $t_{*} \geq 0$, we define $y(\theta)=y_{k, j}^{t_{*}}(\theta)=\left(s_{k, j}\left(\theta ; t_{*}\right)\right.$, $\left.i_{k, j}\left(\theta ; t_{*}\right), r_{k, j}\left(\theta ; t_{*}\right)\right)^{T}$ and $g=g_{k, j}=\left(g_{S}, g_{I}, g_{R}\right)^{T}$, where $y:\left[0, \tau_{k, j}\right] \rightarrow \mathbb{R}^{3}, g: \mathbb{R}^{3} \rightarrow \mathbb{R}^{3}$ and

$$
\begin{aligned}
g_{S}(y) & =-\lambda_{k, j}^{T}\left(y_{1}, y_{2}, y_{3}\right), \\
g_{I}(y) & =\lambda_{k, j}^{T}\left(y_{1}, y_{2}, y_{3}\right)-\mu_{k, j}^{T} y_{2}, \\
g_{R}(y) & =\mu_{k, j}^{T} y_{2} .
\end{aligned}
$$

Then for each $j, k$ and $t_{*}$, system

$$
\begin{aligned}
y^{\prime}(\theta) & =g(y(\theta)), \\
y(0) & =y_{0}
\end{aligned}
$$

is a compact form of system $\left(T_{k, j}\right)$ with initial values $\left(I V T_{k, j}\right)$ for $y_{0}=\left(\alpha_{k, j} S_{k}\left(t_{*}\right)\right.$, $\left.\alpha_{k, j} I_{k}\left(t_{*}\right), \alpha_{k, j} R_{k}\left(t_{*}\right)\right)^{T}$. The following theorem concerns with fundamental properties of 
the solution $y\left(\theta ; 0, y_{0}\right)$ of system (4.5). The statement is based on the Lipschitz condition (Lip) which was defined in Chapter 2.

Theorem 4.6. Assume that $\lambda_{k, j}^{T}$ possesses the Lipschitz condition (Lip) for any $j, k \in$ $\{1, \ldots, r\}, j \neq k$, on each bounded subset of $\mathbb{R}^{3}$. Moreover, suppose that $\lambda_{k, j}^{T}\left(z_{1}, z_{2}, z_{3}\right)$ $\geq 0$ and $\lambda_{k, j}^{T}\left(0, z_{2}, z_{3}\right)=0$ hold for $z_{1}, z_{2}, z_{3} \geq 0$. Then there exists a unique solution of system (4.5) for $\theta \in[0, \infty)$, which continuously depends on the initial data. Furthermore, the inequality

$$
0 \leq y\left(\theta ; 0, y_{0}\right) \leq \sqrt{3}\left|y_{0}\right|
$$

holds componentwise, which means that solutions are bounded and remain nonnegative for nonnegative initial data.

Proof. By the definition of $g$ we get that $g$ satisfies (Lip). The Lipschitz condition guarantees the existence of a unique solution, which continuously depends on the initial data ([22]). In the model setup we have seen that $n_{k, j}\left(\theta ; t_{*}\right)$ is constant for all $\theta$ in the maximal interval of existence, moreover from the nonnegativity condition on $\lambda_{k, j}^{T}$ it follows that nonnegative initial data give rise to nonnegative solution. Hence we obtain

$$
\begin{aligned}
0 & \leq n_{k, j}\left(0 ; t_{*}\right)=n_{k, j}\left(\theta ; t_{*}\right), \\
0 & \leq s_{k, j}\left(0 ; t_{*}\right)+i_{k, j}\left(0 ; t_{*}\right)+r_{k, j}\left(0 ; t_{*}\right)=s_{k, j}\left(\theta ; t_{*}\right)+i_{k, j}\left(\theta ; t_{*}\right)+r_{k, j}\left(\theta ; t_{*}\right) \\
& =\alpha_{k, j}\left(S_{k}\left(t_{*}\right)+I_{k}\left(t_{*}\right)+R_{k}\left(t_{*}\right)\right), \\
0 & \leq s_{k, j}\left(\theta ; t_{*}\right), i_{k, j}\left(\theta ; t_{*}\right), r_{k, j}\left(\theta ; t_{*}\right) \leq \alpha_{k, j}\left(S_{k}\left(t_{*}\right)+I_{k}\left(t_{*}\right)+R_{k}\left(t_{*}\right)\right),
\end{aligned}
$$

where we used $\left(I V T_{k, j}\right)$. With the definitions of $y$ and $y_{0},(4.6)$ implies that the inequality

$$
\begin{aligned}
0 & \leq\left(y\left(\theta ; 0, y_{0}\right)\right)_{1},\left(y\left(\theta ; 0, y_{0}\right)\right)_{2},\left(y\left(\theta ; 0, y_{0}\right)\right)_{3} \leq\left(y_{0}\right)_{1}+\left(y_{0}\right)_{2}+\left(y_{0}\right)_{3} \\
& \leq \sqrt{3} \sqrt{\left(\left(y_{0}\right)_{1}\right)^{2}+\left(\left(y_{0}\right)_{2}\right)^{2}+\left(\left(y_{0}\right)_{3}\right)^{2}}
\end{aligned}
$$

holds on the maximal interval of existence, where we used the arithmetic-quadratic mean inequality. We conclude that the solution is bounded and thus exists for $\theta \in[0, \infty)$.

For every $j, k \in\{1, \ldots, r\}, j \neq k$, let $h_{k, j}: \mathbb{R}^{3 r} \rightarrow \mathbb{R}^{3}$ be defined by $h_{k, j}=\left(h_{S, k, j}, h_{I, k, j}\right.$, $\left.h_{R, k, j}\right)^{T}$ and

$$
\begin{aligned}
h_{S, k, j}(v) & =\alpha_{k, j} v_{3 k-2}, \\
h_{I, k, j}(v) & =\alpha_{k, j} v_{3 k-1}, \\
h_{R, k, j}(v) & =\alpha_{k, j} v_{3 k} .
\end{aligned}
$$

The feasible phase space is the nonnegative cone $C_{+}=C\left([-\tau, 0], \mathbb{R}_{+}^{3 r}\right)$ of the Banach space of continuous functions from $[-\tau, 0]$ to $\mathbb{R}^{3 r}$ with the supremum norm. For $\phi \in C_{+}$, 
we use the notation $y_{\phi\left(-\tau_{k, j}\right)}(\theta)=y\left(\theta ; 0, h_{k, j}\left(\phi\left(-\tau_{k, j}\right)\right)\right) \in \mathbb{R}^{3}$. Furthermore, we define $W_{k}: C_{+} \rightarrow \mathbb{R}^{3 r}$ as

$$
\left(\begin{array}{c}
\left(W_{k}(\phi)\right)_{3 j-2} \\
\left(W_{k}(\phi)\right)_{3 j-1} \\
\left(W_{k}(\phi)\right)_{3 j}
\end{array}\right)= \begin{cases}y_{\phi\left(-\tau_{k, j}\right.}\left(\tau_{k, j}\right) & \text { if } j \neq k, \\
0 & \text { if } j=k,\end{cases}
$$

$j \in\{1, \ldots, r\}$. Let $x(t)=\left(S_{1}(t), I_{1}(t), R_{1}(t), \ldots, S_{r}(t), I_{r}(t), R_{r}(t)\right)^{T}$ for $t \geq 0$, and $f=\left(f_{S, 1}, f_{I, 1}, f_{R, 1}, \ldots, f_{S, r}, f_{I, r}, f_{R, r}\right)^{T}$ with

$$
\begin{aligned}
& f_{S, j}(x)=-\Lambda_{j}\left(x_{3 j-2}, x_{3 j-1}, x_{3 j}\right)-\left(\sum_{k=1}^{r} \alpha_{j, k}\right) x_{3 j-2}, \\
& f_{I, j}(x)=\Lambda_{j}\left(x_{3 j-2}, x_{3 j-1}, x_{3 j}\right)-\mu_{j} x_{3 j-1}-\left(\sum_{k=1}^{r} \alpha_{j, k}\right) x_{3 j-1}, \\
& f_{R, j}(x)=\mu_{j} x_{3 j-1}-\left(\sum_{k=1}^{r} \alpha_{j, k}\right) x_{3 j}
\end{aligned}
$$

for $j \in\{1, \ldots, r\}$. Clearly the union of systems $\left(L_{j}\right)$ with initial conditions $\left(I V L_{j}\right)$, $j \in\{1, \ldots, r\}$, can be written in a closed form as

$$
\begin{aligned}
x^{\prime}(t) & =f(x(t))+\sum_{k=1}^{r} W_{k}\left(x_{t}\right)=: \mathcal{F}\left(x_{t}\right), \\
x_{0} & =\Phi,
\end{aligned}
$$

where $x: \mathbb{R}_{+} \rightarrow \mathbb{R}^{3 r}, f: \mathbb{R}^{3 r} \rightarrow \mathbb{R}^{3 r}, \mathcal{F}: C_{+} \rightarrow \mathbb{R}^{3 r}$, and $\Phi \in C_{+}$is defined as $\Phi:=$ $\left(\varphi_{S, 1}, \varphi_{I, 1}, \varphi_{R, 1}, \ldots, \varphi_{S, n}, \varphi_{I, n}, \varphi_{R, n}\right)^{T}$.

We note that the compact form of the multiregional model shows similarities with the general systems (2.9) and (2.10) defined in Section 2.3, Chapter 2. Thus, it is reasonable to expect that results on the existence, uniqueness and nonnegativity of the solution of this model can be derived using analogous methods as in Chapter 2. These issues are detailed in the next section.

\subsubsection{Basic properties of the model}

This section is devoted to the proof of the general existence and uniqueness result of system (4.7), moreover we will show that, under reasonable conditions on the incidence functions, solutions are nonnegative. First we prove that if we assume that $\Lambda_{j}$ and $\lambda_{k, j}^{T}$ possess the Lipschitz property $\left(\right.$ Lip), then $\mathcal{F}$ also satisfies the Lipschitz condition $\left(\right.$ Lip $\left.^{C}\right)$ which has been defined in Chapter 2 for autonomous systems. 
Lemma 4.7. Suppose that for all $j, k \in\{1, \ldots, r\}, j \neq k, \Lambda_{j}$ and $\lambda_{k, j}^{T}$ possess the Lipschitz property, moreover $\lambda_{k, j}^{T}\left(z_{1}, z_{2}, z_{3}\right) \geq 0$ and $\lambda_{k, j}^{T}\left(0, z_{2}, z_{3}\right)=0$ hold for $z_{1}, z_{2}, z_{3} \geq 0$. Then $\mathcal{F}$ satisfies the Lipschitz condition on each bounded subset of $C_{+}$.

Proof. We claim that for every $M>0$ there exists a constant $K=K(M)$ such that the inequality $|\mathcal{F}(\phi)-\mathcal{F}(\psi)| \leq K\|\phi-\psi\|$ holds for every $\phi, \psi \in C_{+}$with $\|\phi\|,\|\psi\| \leq M$.

Fix indices $j, k \in\{1, \ldots, r\}, j \neq k$. For $\|\psi\| \leq M$ it holds component-wise that $0 \leq \psi\left(-\tau_{k, j}\right) \leq M$, so due to the continuity of $h_{k, j}$, there exists a constant $L_{k, j}^{h}(M)$ such that $0 \leq h_{k, j}\left(\psi\left(-\tau_{k, j}\right)\right) \leq L_{k, j}^{h}$ is satisfied component-wise. For $y_{0}=h_{k, j}\left(\psi\left(-\tau_{k, j}\right)\right)$ Theorem 4.6 implies that there exists a $J_{k, j}=J_{k, j}\left(L_{k, j}^{h}\right)=J_{k, j}(M)$ such that the inequality $\left|y_{\psi\left(-\tau_{k, j}\right)}(\theta)\right| \leq J_{k, j}$ holds for $\theta \in[0, \tau]$ (for instance, one can let $J_{k, j}=\sqrt{3} L_{k, j}^{h}$ ).

The Lipschitz property of $h_{k, j}$ follows from its definition. We assumed that $\lambda_{k, j}^{T}$ is Lipschitz continuous, this implies the Lipschitz continuity of $g$. Let $K_{k, j}^{h}=K_{k, j}^{h}(M)$ be the Lipschitz constant of $h_{k, j}$ on the set $\left\{v \in \mathbb{R}^{3 r}:|v| \leq M\right\}$, we denote the Lipschitz constant of $g=g_{k, j}$ on the set $\left\{v \in \mathbb{R}^{3}:|v| \leq J_{k, j}\right\}$ by $K_{k, j}^{g}=K_{k, j}^{g}(J)=K_{k, j}^{g}(M)$. For any $\|\phi\|,\|\psi\| \leq M$, it holds that $\left|\phi\left(-\tau_{k, j}\right)\right|,\left|\psi\left(-\tau_{k, j}\right)\right| \leq M$. Since solutions of (4.5) can be expressed as $y\left(\theta ; 0, y_{0}\right)=y_{0}+\int_{0}^{\theta} g\left(y\left(u ; 0, y_{0}\right)\right) \mathrm{d} u$, we have

$$
\begin{aligned}
\left|y_{\phi\left(-\tau_{k, j}\right)}(\theta)-y_{\psi\left(-\tau_{k, j}\right)}(\theta)\right|= & \mid h_{k, j}\left(\phi\left(-\tau_{k, j}\right)\right)+\int_{0}^{\theta} g\left(y_{\phi\left(-\tau_{k, j}\right)}(u)\right) \mathrm{d} u \\
& -\left(h_{k, j}\left(\psi\left(-\tau_{k, j}\right)\right)+\int_{0}^{\theta} g\left(y_{\psi\left(-\tau_{k, j}\right)}(u)\right) \mathrm{d} u\right) \mid \\
\leq & \left|h_{k, j}\left(\phi\left(-\tau_{k, j}\right)\right)-h_{k, j}\left(\psi\left(-\tau_{k, j}\right)\right)\right| \\
& +\int_{0}^{\theta}\left|g\left(y_{\phi\left(-\tau_{k, j}\right)}(u)\right)-g\left(y_{\psi\left(-\tau_{k, j}\right)}(u)\right)\right| \mathrm{d} u \\
\leq & K_{k, j}^{h}|| \phi-\psi \|+\int_{0}^{\theta} K_{k, j}^{g}\left|y_{\phi\left(-\tau_{k, j}\right)}(u)-y_{\psi\left(-\tau_{k, j}\right)}(u)\right| \mathrm{d} u
\end{aligned}
$$

for $\theta \in[0, \tau]$. By the definition

$$
\Gamma(\theta)=\left|y_{\phi\left(-\tau_{k, j}\right)}(\theta)-y_{\psi\left(-\tau_{k, j}\right)}(\theta)\right|
$$

for $\theta \in[0, \tau]$, it follows that

$$
\Gamma(\theta) \leq K_{k, j}^{h}\|\phi-\psi\|+K_{k, j}^{g} \int_{0}^{\theta} \Gamma(u) \mathrm{d} u,
$$

and using Gronwall's inequality we derive

$$
\Gamma(\theta) \leq K_{k, j}^{h}\|\phi-\psi\| e^{K_{k, j}^{g} \theta} .
$$


Applying the definition of $W_{k}$, we arrive to the inequality

$$
\begin{aligned}
\left|\left(\begin{array}{c}
\left(W_{k}(\phi)\right)_{3 j-2} \\
\left(W_{k}(\phi)\right)_{3 j-1} \\
\left(W_{k}(\phi)\right)_{3 j}
\end{array}\right)-\left(\begin{array}{c}
\left(W_{k}(\psi)\right)_{3 j-2} \\
\left(W_{k}(\psi)\right)_{3 j-1} \\
\left(W_{k}(\psi)\right)_{3 j}
\end{array}\right)\right| & =\left|y_{\phi\left(-\tau_{k, j}\right)}\left(\tau_{k, j}\right)-y_{\psi\left(-\tau_{k, j}\right)}\left(\tau_{k, j}\right)\right| \\
& \leq K_{k, j}^{h} e^{K_{k, j}^{g} \tau_{k, j}}\|\phi-\psi\|,
\end{aligned}
$$

where we used (4.8) at $\theta=\tau_{k, j}$. It follows that $W_{k}$ satisfies the Lipschitz condition for any $k \in\{1, \ldots, r\}$, and $K^{W_{k}}=K^{W_{k}}(M)=\sqrt{\sum_{\substack{j=1 \\ j \neq k}}^{r}\left(K_{k, j}^{h} e^{K_{k, j}^{g} \tau_{k, j}}\right)^{2}}$ is a suitable choice for the Lipschitz constant.

Finally, the assumption that $\Lambda_{j}$ is Lipschitz continuous for any $j \in\{1, \ldots, r\}$ implies the Lipschitz continuity of $f$, so let $K^{f}=K^{f}(M)$ be the Lipschitz constant of $f$ on the set $\left\{v \in \mathbb{R}^{3 r}:|v| \leq M\right\}$. Then for any $\|\phi\|,\|\psi\| \leq M$ it holds that $|\phi(0)|,|\psi(0)|,|\phi(-\tau)|$, $|\psi(-\tau)| \leq M$, thus we arrive to

$$
\begin{aligned}
|\mathcal{F}(\phi)-\mathcal{F}(\psi)| & \leq|f(\phi(0))-f(\psi(0))|+\sum_{k=1}^{r}\left|W_{k}(\phi)-W_{k}(\psi)\right| \\
& \leq K^{f}|| \phi-\psi\left\|+\sum_{k=1}^{r} K^{W_{k}}\right\| \phi-\psi \| .
\end{aligned}
$$

Hence $K^{f}+\sum_{k=1}^{r} \sqrt{\sum_{\substack{j=1 \\ j \neq k}}^{r}\left(K_{k, j}^{h} e^{K_{k, j}^{g} \tau_{k, j}}\right)^{2}}$ is a suitable choise for $K$, the Lipschitz constant of $\mathcal{F}$ on the set $\left\{\psi \in C_{+}:\|\psi\| \leq M\right\}$.

Proposition 4.8. Assume that for any $j, k \in\{1, \ldots, r\}, j \neq k, \Lambda_{j}\left(z_{1}, z_{2}, z_{3}\right) \geq 0$, $\Lambda_{j}\left(0, z_{2}, z_{3}\right)=0, \lambda_{k, j}^{T}\left(z_{1}, z_{2}, z_{3}\right) \geq 0$ and $\lambda_{k, j}^{T}\left(0, z_{2}, z_{3}\right)=0$ hold for $z_{1}, z_{2}, z_{3} \geq 0$. Then for any $\Phi \in C_{+}$, the solution of system (4.7) is nonnegative, where it exists.

Proof. As proved in Theorem 4.6, solutions of system (4.5) are nonnegative. For each $k \in\{1, \ldots, r\}$, we define $\bar{W}_{k}: \mathbb{R}^{3 r} \times \cdots \times \mathbb{R}^{3 r} \rightarrow \mathbb{R}^{3 r}$ as

$$
\left(\begin{array}{c}
\left(\bar{W}_{k}\left(w_{1}, \ldots, w_{r}\right)\right)_{3 j-2} \\
\left(\bar{W}_{k}\left(w_{1}, \ldots, w_{r}\right)\right)_{3 j-1} \\
\left(\bar{W}_{k}\left(w_{1}, \ldots, w_{r}\right)\right)_{3 j}
\end{array}\right)= \begin{cases}y\left(\tau_{k, j} ; 0, h_{k, j}\left(w_{j}\right)\right) & \text { if } j \neq k, \\
0 & \text { if } j=k\end{cases}
$$

where $j \in\{1, \ldots, r\}, w_{1}, \ldots, w_{r} \in \mathbb{R}^{3 r}$. Consider the following differential equation with multiple discrete delays

$$
\begin{aligned}
x^{\prime}(t) & =f(x(t))+\sum_{k=1}^{r} \bar{W}_{k}\left(x\left(t-\tau_{k, 1}\right), \ldots, x\left(t-\tau_{k, r}\right)\right), \\
x_{0} & =\Phi .
\end{aligned}
$$


It is not hard to see that (4.9) is equivalent to system (4.7), and we claim that the solution of system (4.9) preserves nonnegativity. Similarly as in Chapter 2 we use Theorem 3.4 in [38] which extends naturally to the case of multiple discrete delays, its conditions are clearly satisfied: $\bar{W}_{k} \geq 0$ holds by the nonnegativity of $y\left(\tau_{k, j} ; 0, h_{k, j}\left(w_{j}\right)\right), j, k \in\{1, \ldots, r\}, j \neq k$, and the assumption on the $\Lambda_{j}$-s guarantees that $f_{l}(x) \geq 0$ is satisfied whenever $x \in \mathbb{R}_{+}^{3 r}$, $x_{l}=0, l \in\{1, \ldots, 3 r\}$. We conclude that for non-negative initial data the solution of system (4.7) remains nonnegative, where it exists.

The assumptions of Lemma 4.7 and Proposition 4.8 on the incidences $\Lambda_{j}\left(S_{j}(t), I_{j}(t)\right.$, $\left.R_{j}(t)\right)$ and $\lambda_{k, j}^{T}\left(s_{k, j}\left(\theta ; t_{*}\right), i_{k, j}\left(\theta ; t_{*}\right), r_{k, j}\left(\theta ; t_{*}\right)\right)$ can be fulfilled by various choices on the type of disease transmission. For instance, let $\beta_{j}>0$ be the transmission rate in region $j$ and let $\beta_{k, j}^{T}>0$ denote the transmission rate during the travel from region $k$ to $j$, $j, k \in\{1, \ldots, r\}, j \neq k$. If we define

$$
\begin{aligned}
\Lambda_{j}(z) & =-\beta_{j} \frac{z_{1}}{z_{1}+z_{2}+z_{3}} z_{2}, \\
\lambda_{k, j}^{T}(z) & =-\beta_{k, j}^{T} \frac{z_{1}}{z_{1}+z_{2}+z_{3}} z_{2},
\end{aligned}
$$

for $z \in \mathbb{R}^{3} \backslash\{0\}$ then $\Lambda_{j}$ and $\lambda_{k, j}^{T}$ obtain the forms

$$
\begin{aligned}
\Lambda_{j}\left(S_{j}, I_{j}, R_{j}\right) & =-\beta_{j} \frac{S_{j}}{N_{j}} I_{j}, \\
\lambda_{k, j}^{T}\left(s_{k, j}, i_{k, j}, r_{k, j}\right) & =-\beta_{k, j}^{T} \frac{s_{k, j}}{n_{k, j}} i_{k, j},
\end{aligned}
$$

which is called standard incidence.

Theorem 4.9. With the incidences $\Lambda_{j}$ and $\lambda_{k, j}^{T}$ defined in (4.10), there exists a unique solution of system (4.7).

Proof. Similarly as in Chapter 2, we refer to Theorem 3.7 from [38] which states that the autonomous functional differential equation of the general form $x^{\prime}(t)=\mathcal{F}\left(x_{t}\right)$ has a unique solution if the condition $\left(\operatorname{Lip}^{C}\right)$, defined in Chapter 2, is satisfied for $\mathcal{F}$. We showed in Lemma 4.7 that the Lipschitz condition for $\mathcal{F}$ follows from the Lipschitz property of the incidences and the nonnegativity condition on $\lambda_{k, j}^{T}$. The latter clearly holds with (4.10), hence it remains to prove that the incidences possess the Lipschitz property ( Lip). As one may observe, the definition of the $\Lambda_{j}$-s and $\lambda_{k, j}^{T}$-s only differ in constant multipliers, hence it is sufficient to prove the Lipschitz condition only for one of them, i.e., for $\Lambda_{1}$. Moreover, we prove this property only on the nonnegative cone $\mathbb{R}_{+}^{3}$, which is invariant under systems 
(4.5) and (4.7) (see Theorem 4.6, Proposition 4.8). For $z, w \in \mathbb{R}_{+}^{3}, z \neq 0, w \neq 0$, by

$$
\begin{aligned}
\left|\Lambda_{1}(z)-\Lambda_{1}(w)\right|= & -\beta_{1} \frac{z_{1} z_{2}}{z_{1}+z_{2}+z_{3}}+\beta_{1} \frac{w_{1} w_{2}}{w_{1}+w_{2}+w_{3}} \mid \\
\leq & \beta_{1}\left(\left|\frac{w_{1} w_{2}}{w_{1}+w_{2}+w_{3}}-\frac{w_{1} z_{2}}{w_{1}+w_{2}+w_{3}}\right|\right. \\
& +\left|\frac{w_{1} z_{2}}{w_{1}+w_{2}+w_{3}}-\frac{w_{1} z_{2}}{w_{1}+z_{2}+w_{3}}\right|+\left|\frac{w_{1} z_{2}}{w_{1}+z_{2}+w_{3}}-\frac{w_{1} z_{2}}{w_{1}+z_{2}+z_{3}}\right| \\
& \left.+\left|\frac{w_{1} z_{2}}{w_{1}+z_{2}+z_{3}}-\frac{w_{1} z_{2}}{z_{1}+z_{2}+z_{3}}\right|+\left|\frac{w_{1} z_{2}}{z_{1}+z_{2}+z_{3}}-\frac{z_{1} z_{2}}{z_{1}+z_{2}+z_{3}}\right|\right) \\
= & \beta_{1}\left(\left|w_{2}-z_{2}\right| \frac{w_{1}}{w_{1}+w_{2}+w_{3}}+\left|z_{2}-w_{2}\right| \frac{w_{1} z_{2}}{\left(w_{1}+w_{2}+w_{3}\right)\left(w_{1}+z_{2}+w_{3}\right)}\right. \\
& +\left|z_{3}-w_{3}\right| \frac{w_{2}}{\left(w_{1}+z_{2}+w_{3}\right)\left(w_{1}+z_{2}+z_{3}\right)} \\
& \left.+\left|z_{1}-w_{1}\right| \frac{z_{2}}{\left(w_{1}+z_{2}+z_{3}\right)\left(z_{1}+z_{2}+z_{3}\right)}+\left|w_{1}-z_{1}\right| \frac{z_{2}}{z_{1}+z_{2}+z_{3}}\right) \\
\leq & \beta_{1}\left(2\left|w_{2}-z_{2}\right|+\left|z_{3}-w_{3}\right|+2\left|z_{1}-w_{1}\right|\right) \\
\leq & 5 \beta_{1}|w-z|_{3}
\end{aligned}
$$

we obtain the Lipschitz constant $K=5 \beta_{1}$, where we used that $\frac{a}{a+b+c} \leq 1$ holds for any $a, b, c \geq 0,(a, b, c) \neq(0,0,0)$.

Remark 4.10. It follows from the proof of Theorem 4.9 that the incidences $\Lambda_{j}$ and $\lambda_{k, j}^{T}$ defined in (4.10) also satisfy the global Lipschitz property, meaning that there is a Lipschitz constant $K$ which is independent of $M$. In this case, the solution of system (4.7) exists on $[0, \infty)$.

Another natural choice for the incidences can be the following: for $z=\left(z_{1}, z_{2}, z_{3}\right) \in \mathbb{R}^{3}$ and for $j, k \in\{1, \ldots, r\}, j \neq k$, let

$$
\begin{gathered}
\Lambda_{j}(z)=-\beta_{j} z_{1} z_{2}, \\
\lambda_{k, j}^{T}(z)=-\beta_{k, j}^{T} z_{1} z_{2},
\end{gathered}
$$

which leads to the mass action-type disease transmission, therefore $\Lambda_{j}$ and $\lambda_{k, j}^{T}$ have the forms

$$
\begin{aligned}
\Lambda_{j}\left(S_{j}, I_{j}, R_{j}\right) & =-\beta_{j} S_{j} I_{j}, \\
\lambda_{k, j}^{T}\left(s_{k, j}, i_{k, j}, r_{k, j}\right) & =-\beta_{k, j}^{T} s_{k, j} i_{k, j} .
\end{aligned}
$$

Theorem 4.11. With incidences $\Lambda_{j}$ and $\lambda_{k, j}^{T}$ defined in (4.11), there exists a unique solution of system (4.7). 
Proof. Similarly as in Theorem 4.9, it suffices to show that $\Lambda_{j}$ and $\lambda_{k, j}^{T}$ satisfy the Lipschitz property, and we detail the proof only for $\Lambda_{1}$ and consider the nonnegative subspace $R_{+}^{3}$. For any $M>0$ and for any $z, w \in \mathbb{R}_{+}^{3}$ such that $|z|_{3},|w|_{3} \leq M$, we obtain

$$
\begin{aligned}
\left|\Lambda_{1}(z)-\Lambda_{1}(w)\right| & =\left|-\beta_{1} z_{1} z_{2}+\beta_{1} w_{1} w_{2}\right| \\
& \leq \beta_{1}\left|z_{1} z_{2}-w_{1} w_{2}\right| \\
& \leq \beta_{1}\left|z_{1} z_{2}-z_{1} w_{2}+z_{1} w_{2}-w_{1} w_{2}\right| \\
& \leq \beta_{1}\left(\left|z_{1} z_{2}-z_{1} w_{2}\right|+\left|z_{1} w_{2}-w_{1} w_{2}\right|\right) \\
& \leq \beta_{1}\left(z_{1}\left|z_{2}-w_{2}\right|+w_{2}\left|z_{1}-w_{1}\right|\right) \\
& \leq 2 M \beta_{1}|w-z|_{3},
\end{aligned}
$$

so we can choose $K(M)=2 M \beta_{1}$.

Remark 4.12. Although the global Lipschitz property does not hold for $\Lambda_{j}$ and $\lambda_{k, j}^{T}$ defined in (4.11), the boundedness of the solution of (4.7) implies its existence on $[0, \infty)$. Indeed, Proposition 4.8 yields nonnegativity, and it follows from the model equations that solutions are bounded above by the total population of $r$ regions $N_{1}(t)+\cdots+N_{r}(t)$, which is constant for all $t \geq 0$ since demographic effects are not incorporated into the model. 


\section{Chapter 5}

\section{Backward bifurcation in SIVS model with immigration of non-infectives}

This chapter investigates how travel-related inflow of individuals (e.g., immigration) affects the bifurcation dynamics of an epidemic model in a single population. A simple SIVS (susceptible-infected-vaccinated-susceptible) disease transmission model with immigration of susceptible and vaccinated individuals is considered, which may undergo two different types of bifurcations, i.e., forward and backward transcritical bifurcations, when the reproduction number equals unity. The difference between the two scenarios lies in the number of positive (endemic) steady states of the model: in contrary to the case of forward bifurcation when positive equilibria can only exist for $\mathcal{R}_{0}>1$, there are two endemic states for some values of $\mathcal{R}_{0}$ less than one when the system exhibits backward bifurcation. After giving an explicit condition for the existence of backward bifurcation and multiple endemic equilibria, we show global stability results for the model and examine in detail how the structure of the bifurcation diagram depends on the immigration.

\subsection{Introduction}

The basic reproduction number $\mathcal{R}_{0}$ is a central quantity in epidemiology, as it determines the average number of secondary infections caused by a typical infected individual introduced into a wholly susceptible population. In epidemic models describing the spread of infectious diseases, the reproduction number works as a threshold quantity for the stability of the disease free equilibrium. The usual situation is that for $\mathcal{R}_{0}<1$ the DFE is the only equilibrium and it is asymptotically stable, but it loses its stability as $\mathcal{R}_{0}$ increases through one, where a stable endemic equilibrium emerges, which depends continuously 


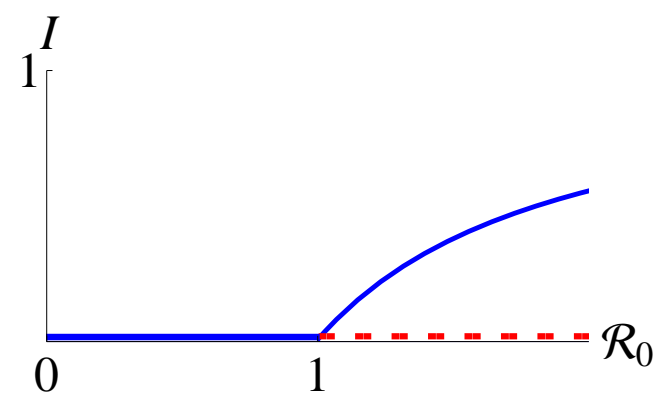

(a) Forward bifurcation.

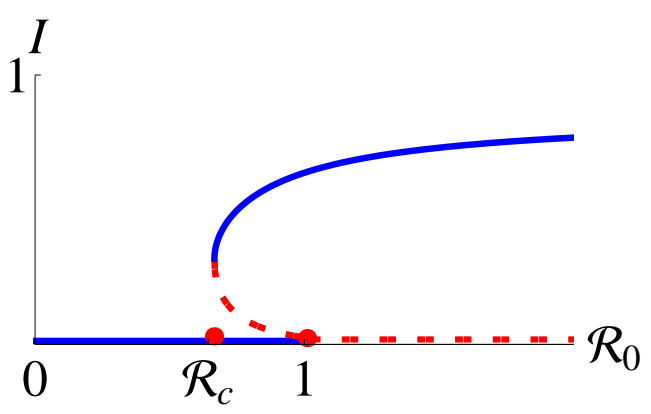

(b) Backward bifurcation.

Figure 5.1: Schematic bifurcation diagrams.

on $\mathcal{R}_{0}$. Such transition of stability between the disease free equilibrium and the endemic equilibrium is called forward bifurcation. However, it is possible to have a very different situation at $\mathcal{R}_{0}=1$, as there might exist positive equilibria also for values of $\mathcal{R}_{0}$ less than one. In this case we say that the model undergoes a backward bifurcation at $\mathcal{R}_{0}=1$, when for values of $\mathcal{R}_{0}$ in an interval to the left of one, multiple positive equilibria coexist, typically one unstable and one stable. The behavior in the change of stability is of particular interest from the perspective of controlling the epidemic: considering $\mathcal{R}_{0}>1$, in order to eradicate the disease it is sufficient to decrease $\mathcal{R}_{0}$ to one if there is a forward bifurcation at $\mathcal{R}_{0}=1$, however it is necessary to bring $\mathcal{R}_{0}$ well below one to eliminate the infection in case of a backward bifurcation. This also implies that the qualitative behavior of a model with backward bifurcation is more complicated than that of a model which undergoes forward bifurcation at $\mathcal{R}_{0}=1$. In the latter case, the infection usually does not persist if $\mathcal{R}_{0}<1$, although with backward bifurcation the presence of a stable endemic equilibrium for $\mathcal{R}_{0}<1$ implies that, even for values of $\mathcal{R}_{0}$ less than one, the epidemic can sustain itself if enough infected individuals are present. The phenomena of forward and backward bifurcations are illustrated by the schematic bifurcation diagrams of Figure 5.1.

Backward bifurcation has been observed in several studies in the recent literature (for an overview see, for instance, [18] and the references therein). The well-known works $[15,19,20]$ consider multi-group epidemic models with asymmetry between groups or multiple interaction mechanisms. Some simple epidemic models of disease transmission in a single population with vaccination of susceptible individuals are presented and analyzed in $[7,8,26,27]$. A basic model can be described by the following system of ordinary 
differential equations:

$$
\begin{aligned}
\dot{S}(t) & =\Lambda(N(t))-\beta(N(t)) S(t) I(t)-(\mu+\phi) S(t)+\gamma I(t)+\theta V(t), \\
\dot{I}(t) & =\beta(N(t)) S(t) I(t)+\sigma \beta(N(t)) V(t) I(t)-(\mu+\gamma) I(t), \\
\dot{V}(t) & =\phi S(t)-\sigma \beta(N(t)) V(t) I(t)-(\mu+\theta) V(t),
\end{aligned}
$$

where $S(t), I(t), V(t)$ and $N(t)$ denote the number of susceptible, infected, vaccinated individuals and the total population, respectively, at time $t$. $\Lambda$ represents the birth function into the susceptible class and $\mu$ is the natural death rate in each class. Disease transmission is modeled by the infection term $\beta(N) S I, \phi$ and $\gamma$ stand for the vaccination rate of susceptible individuals and the recovery rate of infected individuals. It is assumed that vaccination loses effect at rate $\theta$, moreover we include the parameter $\sigma, 0 \leq \sigma \leq 1$, to incorporate that vaccination may reduce but not completely eliminate susceptibility to infection. With certain conditions on the birth function $\Lambda$, system (5.1) can be reduced to a two-dimensional system, of which a complete qualitative analysis including a condition for the existence of backward bifurcation has been derived in [7].

We can extend model (5.1) by considering a population that resides in two regions which are connected by transportation. Assuming it takes $\tau>0$ units of time to complete the travel between the regions, a system of delay differential equations can be formulated as

$$
\begin{aligned}
\dot{S}_{1}(t)= & \Lambda_{1}\left(N_{1}(t)\right)-\beta_{1}\left(N_{1}(t)\right) S_{1}(t) I_{1}(t)-\left(\mu_{1}+\phi_{1}\right) S_{1}(t)+\gamma_{1} I_{1}(t)+\theta_{1} V_{1}(t) \\
& -\alpha_{1}^{S} S_{1}(t)+\alpha_{2}^{S} S_{2}(t-\tau) \\
\dot{I}_{1}(t)= & \beta_{1}\left(N_{1}(t)\right) S_{1}(t) I_{1}(t)+\sigma_{1} \beta_{1}\left(N_{1}(t)\right) V_{1}(t) I_{1}(t)-\left(\mu_{1}+\gamma_{1}\right) I_{1}(t) \\
& -\alpha_{1}^{I} I_{1}(t)+\alpha_{2}^{I} I_{2}(t-\tau), \\
\dot{V}_{1}(t)= & \phi_{1} S_{1}(t)-\sigma_{1} \beta_{1}\left(N_{1}(t)\right) V_{1}(t) I_{1}(t)-\left(\mu_{1}+\theta_{1}\right) V_{1}(t) \\
& -\alpha_{1}^{V} V_{1}(t)+\alpha_{2}^{V} V_{2}(t-\tau) \\
\dot{S}_{2}(t)= & \Lambda_{2}\left(N_{2}(t)\right)-\beta_{2}\left(N_{2}(t)\right) S_{2}(t) I_{2}(t)-\left(\mu_{2}+\phi_{2}\right) S_{2}(t)+\gamma_{2} I_{2}(t)+\theta_{2} V_{2}(t) \\
& -\alpha_{2}^{S} S_{2}(t)+\alpha_{1}^{S} S_{1}(t-\tau), \\
\dot{I}_{2}(t)= & \beta_{2}\left(N_{2}(t)\right) S_{2}(t) I_{2}(t)+\sigma_{2} \beta_{2}\left(N_{2}(t)\right) V_{2}(t) I_{2}(t)-\left(\mu_{2}+\gamma_{2}\right) I_{2}(t) \\
& -\alpha_{2}^{I} I_{2}(t)+\alpha_{1}^{I} I_{1}(t-\tau), \\
\dot{V}_{2}(t)= & \phi_{2} S_{2}(t)-\sigma_{2} \beta_{2}\left(N_{2}(t)\right) V_{2}(t) I_{2}(t)-\left(\mu_{2}+\theta_{2}\right) V_{2}(t) \\
& -\alpha_{2}^{V} V_{2}(t)+\alpha_{1}^{V} V_{1}(t-\tau)
\end{aligned}
$$

to describe the spread of an infectious disease in the two subpopulations with vaccination. Lower indices 1 and 2 are introduced to label model compartments and parameters in region 1 and 2, respectively. We assume that susceptible, infected and recovered individuals of 
region $j$ travel by rates $\alpha_{j}^{S}, \alpha_{j}^{I}$ and $\alpha_{j}^{R}$ from region $j$ to region $k$, where $j, k \in\{1,2\}$ and $j \neq k$. In the special case when transportation is one-directional (for instance, there is no connection from region 1 to region 2 and $\alpha_{1}^{S}=0, \alpha_{1}^{I}=0$ and $\alpha_{1}^{V}=0$ ), the equations of $S_{2}, I_{2}$ and $R_{2}$ are independent of the other three equations in system (5.2a)-(5.2b). If the subsystem $(5.2 \mathrm{~b})$ attains a unique globally attracting equilibrium with $\lim _{t \rightarrow \infty} S_{2}(t)=$ $S_{2}^{\infty}, \lim _{t \rightarrow \infty} I_{2}(t)=I_{2}^{\infty}$ and $\lim _{t \rightarrow \infty} V_{2}(t)=V_{2}^{\infty}$, then it follows that system (5.2a) is asymptotically autonomous with the limiting system

$$
\begin{aligned}
\dot{S}_{1}(t)= & \Lambda_{1}\left(N_{1}(t)\right)-\beta_{1}\left(N_{1}(t)\right) S_{1}(t) I_{1}(t)-\left(\mu_{1}+\phi_{1}\right) S_{1}(t) \\
& +\gamma_{1} I_{1}(t)+\theta_{1} V_{1}(t)+\alpha_{2}^{S} S_{2}^{\infty}, \\
\dot{I}_{1}(t)= & \beta_{1}\left(N_{1}(t)\right) S_{1}(t) I_{1}(t)+\sigma_{1} \beta_{1}\left(N_{1}(t)\right) V_{1}(t) I_{1}(t)-\left(\mu_{1}+\gamma_{1}\right) I_{1}(t)+\alpha_{2}^{I} I_{2}^{\infty}, \\
\dot{V}_{1}(t)= & \phi_{1} S_{1}(t)-\sigma_{1} \beta_{1}\left(N_{1}(t)\right) V_{1}(t) I_{1}(t)-\left(\mu_{1}+\theta_{1}\right) V_{1}(t)+\alpha_{2}^{V} V_{2}^{\infty} .
\end{aligned}
$$

The aim of this chapter is to investigate system (5.3) with the additional assumption that $\alpha_{2}^{I}=0$. In this case, system (5.3) allows us to describe and analyze the spread of an epidemic in a single population, where demographic effects - such as immigration of noninfected individuals - are incorporated into the model. The assumption that there is no inflow of infected individuals into the population is realistic if one considers a pandemic situation when entry screening is applied as control policy upon the arrival of passengers to a country. On the other hand, individuals who are showing symptoms like fever or are in pain might decide not to travel anyway. The model we study generalizes the above presented vaccination model (5.1) by incorporating the possibility of immigration, and we investigate how immigration changes the bifurcation behavior.

\subsection{Model description}

A general vaccination model with immigration of non-infected individuals can be described by the system

$$
\begin{aligned}
\dot{S}(t) & =\Lambda(N(t))-\beta(N(t)) S(t) I(t)-(\mu+\phi) S(t)+\gamma I(t)+\theta V(t)+\eta, \\
\dot{I}(t) & =\beta(N(t)) S(t) I(t)+\sigma \beta(N(t)) V(t) I(t)-(\mu+\gamma) I(t), \\
\dot{V}(t) & =\phi S(t)-\sigma \beta(N(t)) V(t) I(t)-(\mu+\theta) V(t)+\omega,
\end{aligned}
$$

where we assume that immigration of susceptible and vaccinated individuals occurs with constant rates $\eta$ and $\omega$, respectively. The other parameters of the model have been described in Section 5.1 and are tabulated in Table 5.1, along with the model variables. For the total population $N(t)$ we obtain

$$
\dot{N}(t)=\Lambda(N(t))-\mu N(t)+\eta+\omega .
$$

The proof of the following proposition is obvious and thus omitted. 


\begin{tabular}{|ll|}
\hline \multicolumn{2}{|c|}{ Variables and key model parameters } \\
\hline$S, I, V, N$ & Susceptible, infected, vaccinated and all individuals \\
$\mu$ & Birth function \\
$\beta$ & Natural death rate \\
$\gamma$ & Transmission rate \\
$\phi$ & Recovery rate of infected individuals \\
$\theta$ & Vaccination rate of susceptible individuals \\
$\sigma$ & Waining rate of vaccine \\
$\eta, \omega$ & Reduction of susceptibility of vaccinated individuals \\
\hline
\end{tabular}

Table 5.1: Variables and parameters of the SIVS model with immigration.

Proposition 5.1. If for the birth function $\Lambda$ it holds that $\Lambda(0)=0, \Lambda^{\prime}(0)>\mu$ and there exists an $x_{*}>0$ such that $\Lambda^{\prime}\left(x_{*}\right)<\mu$, moreover $\Lambda^{\prime}(x)>0$ and $\Lambda^{\prime \prime}(x)<0$ for all $x>0$, then for any $\eta, \omega \geq 0$ there exists a unique positive solution of $\Lambda(x)=\mu x-\eta-\omega$.

The conditions of the last proposition on the birth function can be satisfied for various definitions of $\Lambda$. An example of the birth function found in the literature is $\Lambda(x)=x \cdot b(x)=$ $x \cdot \frac{1}{c+d x}$ with $c, d>0$ and $c<\frac{1}{\mu}$, where the function $b(x)$ is known as the Beverton-Holt function.

We define the population carrying capacity $K=K(\Lambda, \mu, \eta, \omega)$ as the unique solution of $\Lambda(x)=\mu x-\eta-\omega$. Note that from $\Lambda(K)=\mu K-\eta-\omega$ it follows that $\mu K-\eta-\omega>0$. We can rewrite equations $(5.4)_{2}$ and $(5.4)_{3}$ in terms of $N(t), I(t)$ and $V(t)$ using $S(t)=$ $N(t)-I(t)-V(t)$ and consider this system as a system of non-autonomous differential equations with non-autonomous term $N(t)$, which is governed by system (5.5). Then, by $\lim _{t \rightarrow \infty} N(t)=K$ we find that system (5.4) is asymptotically autonomous with the limiting system

$$
\begin{aligned}
\dot{I}(t) & =\beta(K-I(t)-(1-\sigma) V(t)) I(t)-(\mu+\gamma) I(t), \\
\dot{V}(t) & =\phi(K-I(t))-\sigma \beta V(t) I(t)-(\mu+\theta+\phi) V(t)+\omega,
\end{aligned}
$$

where $\beta=\beta(K)$. In what follows we focus on the mathematical analysis of system (5.6), then we use the theory of asymptotically autonomous systems [31, 43, 44] to obtain information on the long-term behavior of solutions of (5.4).

\subsection{Basic properties of the model}

The existence and uniqueness of solutions of system (5.6) follows from fundamental results for ODEs. Since $K$ was defined as the carrying capacity of the population, it is biologically 
meaningful to assume that for the initial conditions of system (5.6) it is satisfied that $0 \leq I(0), V(0), I(0)+V(0) \leq K$.

Proposition 5.2. If for initial values $I(0)$ and $V(0)$ it holds that $0 \leq I(0), V(0), I(0)+$ $V(0) \leq K$, then $0 \leq I(t), V(t), I(t)+V(t) \leq K$ is satisfied for all $t>0$.

Proof. If $I(t)=0$ then $\dot{I}(t)=0$, which yields that for nonnegative initial conditions $I$ never becomes negative. If $V(t)=0$ when $0 \leq I(t) \leq K$, then $\dot{V}(t) \geq \omega \geq 0$, thus solutions never cross the line $V=0$ from the inside of the region $R: 0 \leq I, V, I+V \leq K$. If $I(t)+V(t)=K$ when $I(t), V(t) \geq 0$, then summing $(5.6)_{1}$ and $(5.6)_{2}$ gives

$$
\dot{I}(t)+\dot{V}(t)=-\mu K-\gamma I(t)-\theta V(t)+\omega
$$

which is negative since $\omega-\mu K$ is non-positive, thus $I(t)+V(t)>K$ is impossible.

The disease free equilibrium of system (5.6) can be obtained as

$$
\bar{V}=\frac{\phi K+\omega}{\mu+\theta+\phi} .
$$

In the initial stage of the epidemic, we can assume that system (5.6) is near the equilibrium $(0, \bar{V})$ and approximate the equation of class $I$ with the linear equation

$$
y(t)=(\beta(K-(1-\sigma) \bar{V})-(\mu+\gamma)) y(t)
$$

where $y: \mathbb{R} \rightarrow \mathbb{R}$. The term $\beta(K-(1-\sigma) \bar{V})$ describes the production of new infections, and $\mu+\gamma$ is the transition term describing changes in state, hence with the formula for the disease free equilibrium $\bar{V}$, we can define the basic reproduction number as

$$
\begin{aligned}
\mathcal{R}_{0} & =\frac{\beta(K-(1-\sigma) \bar{V})}{\mu+\gamma} \\
& =\frac{\beta}{\mu+\gamma}\left(\frac{K \cdot(\mu+\theta+\sigma \phi)}{\mu+\theta+\phi}-\frac{(1-\sigma) \omega}{\mu+\theta+\phi}\right) .
\end{aligned}
$$

The following proposition shows that $\mathcal{R}_{0}$ works as a threshold quantity for the stability of the disease free equilibrium of system (5.6).

Proposition 5.3. The disease free equilibrium of system (5.6) is asymptotically stable if $\mathcal{R}_{0}<1$ and unstable if $\mathcal{R}_{0}>1$.

Proof. The stability of the zero steady state of system (5.7) is determined by the sign of $\beta(K-(1-\sigma) \bar{V})-(\mu+\gamma)$, which coincides with the sign of $\mathcal{R}_{0}-1$. This means that the zero solution of (5.7) is asymptotically stable if $\mathcal{R}_{0}<1$ and unstable if $\mathcal{R}_{0}>1$. This statement extends to the nonlinear system (5.6) by the principle of linearized stability. 


\subsection{Endemic equilibria}

The problem of finding equilibrium $(\hat{I}, \hat{V})$ for system (5.6) yields the two-dimensional system

$$
\begin{aligned}
& 0=\beta(K-\hat{I}-(1-\sigma) \hat{V}) \hat{I}-(\mu+\gamma) \hat{I} \\
& 0=\phi(K-\hat{I})-\sigma \beta \hat{V} \hat{I}-(\mu+\theta+\phi) \hat{V}+\omega
\end{aligned}
$$

The existence of a unique disease free equilibrium has been proved, so now we focus on finding endemic equilibria $(\hat{I}, \hat{V})$ with $\hat{I}>0$. From $(5.9)_{1}$ we obtain the formula

$$
\hat{V}=\frac{\beta(K-\hat{I})-(\mu+\gamma)}{\beta(1-\sigma)},
$$

then by substituting $\hat{V}$ into $(5.9)_{2}$ it follows from straightforward computations that

$$
A \hat{I}^{2}+B \hat{I}+C=0
$$

should hold for $\hat{I}$, where

$$
\begin{aligned}
& A=\sigma \beta \\
& B=(\mu+\theta+\sigma \phi)+\sigma(\mu+\gamma)-\sigma \beta K \\
& C=\frac{(\mu+\gamma)(\mu+\theta+\phi)}{\beta}-(\mu+\theta+\sigma \phi) K+(1-\sigma) \omega .
\end{aligned}
$$

We note that $\beta C=\left(1-\mathcal{R}_{0}\right)(\gamma+\mu)(\mu+\phi+\theta)$ and we characterize the number of solutions of the equilibrium condition (5.11).

Proposition 5.4. If $\mathcal{R}_{0}>1$ then there exists a unique positive equilibrium $\hat{I}=$ $\frac{-B+\sqrt{B^{2}-4 A C}}{2 A}$.

Proof. If $C<0$, or equivalently, $\mathcal{R}_{0}>1$, then the equilibrium condition (5.11) has a unique positive solution, which can be obtained as $\hat{I}=\frac{-B+\sqrt{B^{2}-4 A C}}{2 A}$.

At $\mathcal{R}_{0}=1$ it holds that $A>0$ and $C=0$, so there exists a unique nonzero solution $\hat{I}=-B / A$ of (5.11), which is positive (and thus, biologically relevant) if and only if $B<0$. Let us now assume that $B$ is negative at $\mathcal{R}_{0}=1$, which also implies that $B^{2}-4 A C=B^{2}>0$. Then there is a positive root of the equilibrium condition at $\mathcal{R}_{0}=1$, and due to the continuous dependence of the coefficients $A, B$ and $C$ on $\beta$ there must be an interval to the left of $\mathcal{R}_{0}=1$ where $B<0$ and $B^{2}-4 A C>0$ still hold. Since $C>0$ whenever $\mathcal{R}_{0}<1$, it follows that on this interval there exist exactly two positive solutions of (5.11) and thus, two endemic equilibria of system (5.6). We denote these equilibria by

$$
\breve{I}_{1}=\frac{-B-\sqrt{B^{2}-4 A C}}{2 A}, \quad \breve{I}_{2}=\frac{-B+\sqrt{B^{2}-4 A C}}{2 A},
$$


and with the aid of formula (5.10), we can derive the $\hat{V}$-components to get the equilibria $\left(\breve{I}_{1}, \breve{V}_{1}\right)$ and $\left(\breve{I}_{2}, \breve{V}_{2}\right)$. With other words, if $B<0$ when $\mathcal{R}_{0}=1$, then system (5.6) has a backward bifurcation at $\mathcal{R}_{0}=1$, since besides the zero equilibrium and the positive equilibrium $\breve{I}_{2}=\frac{-B+\sqrt{B^{2}-4 A C}}{2 A}$ (which both exist for $\mathcal{R}_{0}>1$ as well), another positive equilibrium emerges when $\mathcal{R}_{0}$ is passing through 1 from the right to the left.

Theorem 5.5. If the condition

$$
\frac{(1-\sigma) \omega}{K}>\frac{(\theta+\mu+\sigma \phi)^{2}-\sigma(\mu+\gamma)(1-\sigma) \phi}{(\theta+\mu+\sigma \phi)+\sigma(\mu+\gamma)}
$$

holds then there is a backward bifurcation at $\mathcal{R}_{0}=1$.

Proof. The condition for the backward bifurcation is that $B<0$ when $\beta$ satisfies $\mathcal{R}_{0}=1$. This can be obtained as an explicit criterion of the parameters: as $B<0$ yields

$$
\sigma \beta K>(\mu+\theta+\sigma \phi)+\sigma(\mu+\gamma)
$$

moreover from $C=0$ we derive

$$
\beta K=\frac{(\mu+\gamma)(\mu+\theta+\phi)}{(\theta+\mu+\sigma \phi)-\frac{(1-\sigma) \omega}{K}}
$$

we get

$$
\begin{aligned}
\frac{\sigma(\mu+\gamma)(\mu+\theta+\phi)}{(\theta+\mu+\sigma \phi)-\frac{(1-\sigma) \omega}{K}}>(\mu+\theta+\sigma \phi)+\sigma(\mu+\gamma) \\
\frac{\sigma(\mu+\gamma)(\mu+\theta+\phi)}{(\theta+\mu+\sigma \phi)+\sigma(\mu+\gamma)}>(\theta+\mu+\sigma \phi)-\frac{(1-\sigma) \omega}{K}, \\
\frac{(1-\sigma) \omega}{K}>(\theta+\mu+\sigma \phi)-\frac{\sigma(\mu+\gamma)(\mu+\theta+\phi)}{(\theta+\mu+\sigma \phi)+\sigma(\mu+\gamma)}, \\
\frac{(1-\sigma) \omega}{K}>\frac{(\theta+\mu+\sigma \phi)^{2}}{(\theta+\mu+\sigma \phi)+\sigma(\mu+\gamma)}-\frac{\sigma(\mu+\gamma)(1-\sigma) \phi}{(\theta+\mu+\sigma \phi)+\sigma(\mu+\gamma)},
\end{aligned}
$$

where we used that $\mu K-\omega>0$.

Theorem 5.6. If condition (5.13) does not hold, then system (5.6) undergoes a forward bifurcation at $\mathcal{R}_{0}=1$. In this case there is no endemic equilibrium for $\mathcal{R}_{0} \in[0,1]$.

Proof. We proceed similarly as in the proof of Theorem 5.5 to find that if

$$
\frac{(1-\sigma) \omega}{K} \leq \frac{(\theta+\mu+\sigma \phi)^{2}-\sigma(\mu+\gamma)(1-\sigma) \phi}{(\theta+\mu+\sigma \phi)+\sigma(\mu+\gamma)}
$$

then $B \geq 0$ when $C=0$, or equivalently, when $\beta$ is set to satisfy $\mathcal{R}_{0}=1$. For $\mathcal{R}_{0}<1$ it holds that $A, C>0$, moreover $B$ is also positive because $B$ is decreasing in $\beta$, these imply that there is no endemic equilibrium on $\mathcal{R}_{0} \in[0,1)$. At $\mathcal{R}_{0}=1$ the equilibrium condition (5.11) becomes $A \hat{I}^{2}+B \hat{I}=0$, and $A>0, B \geq 0$ give that (5.11) has only non-positive 
solutions. However, we know from Proposition 5.4 that there is a positive solution of (5.11) for $\mathcal{R}_{0}>1$, thus we conclude that if the condition (5.13) does not hold, then system (5.6) undergoes a forward bifurcation at $\mathcal{R}_{0}=1$, where a single endemic equilibrium emerges when $\mathcal{R}_{0}$ exceeds 1 .

If (5.13) is satisfied, then there is an interval to the left of $\mathcal{R}_{0}=1$ where there exist positive equilibria. In what follows we determine the left endpoint of this interval. Let us assume that there is a backward bifurcation at $\mathcal{R}_{0}=1$. We define

$$
\begin{aligned}
U & =(\theta+\mu+\sigma \phi)-\frac{(1-\sigma) \omega}{K}, \\
x & =\frac{(1-\sigma) \omega}{K}+\sigma(\mu+\gamma), \\
W & =-x+\sigma \frac{(\gamma+\mu)(\mu+\phi+\theta)}{U} .
\end{aligned}
$$

Note that $x$ and $U$ are positive since $\mu K-\omega>0$ by assumption. The condition for the backward bifurcation can be obtained as

$$
W>U
$$

which also yields the positivity of $W$. We let

$$
\mathcal{R}_{c}=\frac{x-U+2 \sqrt{U W}}{(\mu+\gamma) \sigma} \cdot \frac{U}{\mu+\theta+\phi}
$$

and claim that it defines the critical value of the reproduction number for which there exist endemic equilibria on the interval $\left[\mathcal{R}_{c}, 1\right]$.

Proposition 5.7. Assume that there is a backward bifurcation at $\mathcal{R}_{0}=1$. With $\mathcal{R}_{c}$ defined in (5.16), only the disease free equilibrium exists if $\mathcal{R}_{0}<\mathcal{R}_{c}$, a positive equilibrium emerges at $\mathcal{R}_{0}=\mathcal{R}_{c}$, and on $\left(\mathcal{R}_{c}, 1\right)$ there exist two distinct endemic equilibria. There also exists a positive equilibrium at $\mathcal{R}_{0}=1$.

Proof. The last statement follows from the fact that at $\mathcal{R}_{0}=1(C=0)$, the single non-zero solution $\hat{I}=\frac{-B}{A}$ of $(5.11)$ is positive since $B<0$. The necessary and sufficient conditions $B<0$ and $B^{2}-4 A C>0$ for the existence of two positive distinct equilibria hold on an interval to the left of $\mathcal{R}_{0}=1$. $B=0$ automatically yields $B^{2}-4 A C<0$ if $\mathcal{R}_{0}<1$, hence it is clear that the condition $B^{2}-4 A C=0$ determines the value of $\mathcal{R}_{0}$ for which the positive equilibria disappear. First, we derive the critical value $\beta_{c}$ of the transmission rate from this equation, then substitute $\beta=\beta_{c}$ into the formula of $\mathcal{R}_{0}$ (5.8) to give the critical value of the reproduction number. Using notations $U, x$ and $W$ introduced in (5.14), we 
reformulate $B$ as $B=U+x-\sigma \beta K$ and $C$ as $C=\frac{(\mu+\gamma)(\mu+\theta+\phi)}{\beta}-U K$. The condition $B^{2}-4 A C=0$ becomes

$$
\begin{aligned}
U^{2}+ & 2 U(x-\beta K \sigma)+(x-\beta K \sigma)^{2}-4 \sigma(\mu+\gamma)(\mu+\theta+\phi)+4 \sigma \beta K U \\
& =U^{2}-2 U(x-\beta K \sigma)+(x-\beta K \sigma)^{2}+4 U x-4 \sigma(\mu+\gamma)(\mu+\theta+\phi) \\
& =U^{2}-2 U(x-\beta K \sigma)+(x-\beta K \sigma)^{2}-4 U W=0,
\end{aligned}
$$

so we obtain the roots

$$
\begin{aligned}
(x-\beta K \sigma)_{1,2} & =\frac{2 U \pm \sqrt{4 U^{2}-4 U^{2}+16 U W}}{2} \\
& =U \pm 2 \sqrt{U W}
\end{aligned}
$$

For the positive root $(x-\beta K \sigma)_{2}$, we get $B=U+(x-\beta K \sigma)_{2}>0$, but we require $B<0$ thus we derive from $x-\beta K \sigma=U-2 \sqrt{U W}$ that

$$
\beta_{c}=\frac{x-U+2 \sqrt{U W}}{K \sigma} .
$$

Substituting $\beta_{c}$ into (5.8) gives

$$
\begin{aligned}
\mathcal{R}_{0}\left(\beta_{c}\right) & =\frac{\beta_{c}}{\mu+\gamma}\left(\frac{K(\mu+\theta+\sigma \phi)}{\mu+\theta+\phi}-\frac{(1-\sigma) \omega}{\mu+\theta+\phi}\right) \\
& =\frac{x-U+2 \sqrt{U W}}{(\mu+\gamma) \sigma} \cdot \frac{U}{\mu+\theta+\phi},
\end{aligned}
$$

which is indeed equal to $\mathcal{R}_{c}$ defined in (5.16).

The condition $\mathcal{R}_{0}=1$ reformulates as $\sigma \beta K=W+x$, so with the aid of (5.15) and the computations

$$
\begin{aligned}
0 & <(\sqrt{U}-\sqrt{W})^{2}, \\
2 \sqrt{U W} & <U+W, \\
x-U+2 \sqrt{U W} & <W+x,
\end{aligned}
$$

it is easy to verify that $\mathcal{R}_{c}<1$. The positivity of $\beta_{c}$, and hence, the positivity of $\mathcal{R}_{c}$ follows from the fact that at $\beta=\beta_{c}$ it should hold that $B<0$, which is only possible if $\beta>0$.

We wish to draw the graph of $\hat{I}$ as a function of $\beta$ to obtain the bifurcation curve. By implicitly differentiating the equilibrium condition (5.11) with respect to $\beta$, we get

$$
\begin{aligned}
& (2 A \hat{I}+B) \frac{d \hat{I}}{d \beta}=-\left(\frac{d A}{d \beta} \hat{I}^{2}+\frac{d B}{d \beta} \hat{I}+\frac{d C}{d \beta}\right) \\
& (2 A \hat{I}+B) \frac{d \hat{I}}{d \beta}=\sigma \hat{I}(K-\hat{I})+\frac{(\gamma+\mu)(\mu+\phi+\theta)}{\beta^{2}}
\end{aligned}
$$


The positivity of the right-hand side follows from $K \geq \hat{I}$, which implies that the term $2 A \hat{I}+$ $B$ has the same sign as $\frac{d \hat{I}}{d \beta}$. If $\mathcal{R}_{0}>1$ then there exists the equilibrium $\breve{I}_{2}=\frac{-B+\sqrt{B^{2}-4 A C}}{2 A}$, and we obtain that $2 A \breve{I}_{2}+B>0$, hence for $\mathcal{R}_{0}>1$ the curve has positive slope. If there is a backward bifurcation at $\mathcal{R}_{0}=1$, then on $\left(\mathcal{R}_{c}, 1\right)$ there exists two positive equilibria $\breve{I}_{2}$ and $\breve{I}_{1}=\frac{-B-\sqrt{B^{2}-4 A C}}{2 A}$ with $\breve{I}_{2}>\breve{I}_{1}$, and since it holds that $2 A \breve{I}_{1}+B<0$, we conclude that on $\left(\mathcal{R}_{c}, 1\right)$ the bifurcation curve has negative slope for the smaller endemic equilibrium and positive slope for the larger one. As a matter of fact, the unstable equilibrium is a saddle point, and thus the system experiences a saddle-node bifurcation.

\subsection{Stability and global behavior}

The stability of the disease free equilibrium has been examined in Section 5.3, so now we derive local stability analysis of endemic equilibria. The Jacobian of the system (5.6) at $(\hat{I}, \hat{V})$ gives

$$
J=\left(\begin{array}{cc}
-\beta \hat{I} & -(1-\sigma) \beta \hat{I} \\
-(\phi+\sigma \beta \hat{V}) & -(\mu+\theta+\phi+\sigma \beta \hat{I})
\end{array}\right),
$$

where we used the identity $\beta(K-\hat{I}-(1-\sigma) \hat{V})=\mu+\gamma$ from (5.9), hence the characteristic equation has the form

$$
a_{2} \lambda^{2}+a_{1} \lambda+a_{0}=0
$$

with

$$
\begin{aligned}
& a_{2}=1, \\
& a_{1}=\beta \hat{I}+(\mu+\theta+\phi+\sigma \beta \hat{I}), \\
& a_{0}=\beta \hat{I}(\mu+\theta+\phi+\sigma \beta \hat{I})-(1-\sigma) \beta \hat{I}(\phi+\sigma \beta \hat{V}) .
\end{aligned}
$$

Theorem 5.8. The endemic equilibrium $(\hat{I}, \hat{V})$ for which $\hat{I}=\breve{I}_{2}$ is locally asymptotically stable where it exists: on $\mathcal{R}_{0} \in(1, \infty)$, and also on $\mathcal{R}_{0} \in\left(\mathcal{R}_{c}, 1\right]$ in case there is a backward bifurcation at $\mathcal{R}_{0}=1$. The endemic equilibrium $(\hat{I}, \hat{V})$ for which $\hat{I}=\breve{I}_{1}$ is unstable where it exists: on $\mathcal{R}_{0} \in\left(\mathcal{R}_{c}, 1\right)$ in case there is a backward bifurcation at $\mathcal{R}_{0}=1$.

Proof. The Routh-Hurwitz stability criterion (for a reference see, for example, [17]) states that for all the solutions of the characteristic equation to have negative real parts, all coefficients must have the same sign. Since $a_{2}$ and $a_{1}$ are positive, the sign of $a_{0}$ determines the stability. For that it holds that

$$
\begin{aligned}
a_{0} & =\beta \hat{I}(\mu+\theta+\phi+\sigma \beta \hat{I})-(1-\sigma) \beta \hat{I}(\phi+\sigma \beta \hat{V}) \\
& =\beta \hat{I}(\mu+\theta+\sigma \phi+2 \sigma \beta \hat{I}-\sigma \beta(\hat{I}+(1-\sigma) \hat{V}),
\end{aligned}
$$

so using $-\beta(\hat{I}+(1-\sigma) \hat{V})=\mu+\gamma-\beta K$ we derive

$$
\begin{aligned}
a_{0} & =\beta \hat{I}(\mu+\theta+\sigma \phi+2 \sigma \beta \hat{I}+\sigma(\mu+\gamma-\beta K)) \\
& =\beta \hat{I}(2 A \hat{I}+B) .
\end{aligned}
$$


For $\mathcal{R}_{0}>1$ the only endemic equilibrium is $\breve{I}_{2}=\frac{-B+\sqrt{B^{2}-4 A C}}{2 A}$, for which $2 A \breve{I}_{2}+B>0$ holds and thus $a_{0}>0$ yields its stability. If there is a backward bifurcation at $\mathcal{R}_{0}=1$, then endemic equilibria exist on $\left(\mathcal{R}_{c}, 1\right]$ as well; here $\breve{I}_{2}$ is again stable for the same reason as above, however $\breve{I}_{1}=\frac{-B-\sqrt{B^{2}-4 A C}}{2 A}$ is unstable since $a_{0}=\beta \breve{I}_{1}\left(A \breve{I}_{1}+B\right)<0$.

With the next theorem we describe the global behavior of solutions of system (5.6).

Theorem 5.9. If there exists no endemic equilibrium, that is, if $\mathcal{R}_{0}<1$ in case of a forward bifurcation and if $\mathcal{R}_{0}<\mathcal{R}_{c}$ in case of a backward bifurcation, then every solution converges to the disease free equilibrium. For $\mathcal{R}_{0}>1$, the unique endemic equilibrium is globally attracting. If there is a backward bifurcation at $\mathcal{R}_{0}=1$ then on $\left(\mathcal{R}_{c}, 1\right)$ there is no globally attracting equilibrium, though every solution approaches an equilibrium.

Proof. We first show that every solution of system (5.6) converges to an equilibrium. In Section 5.3 we have proved that the region $R: 0 \leq I, V, I+V \leq K$ is positively invariant for the solutions of system (5.6). We take the $C^{1}$ function $\varphi(I, V)=1 / I$, which does not change sign on $R$ to show that system (5.6) has no periodic solutions lying entirely within the region $R$. The computation

$$
\begin{gathered}
\frac{\partial}{\partial I} \frac{\beta(K-I-(1-\sigma) V) I-(\mu+\gamma) I}{I}+\frac{\partial}{\partial V} \frac{\phi(K-I)-\sigma \beta V I-(\mu+\theta+\phi) V+\omega}{I} \\
\quad=-\beta-\sigma \beta-\frac{\mu+\theta+\phi}{I}<0
\end{gathered}
$$

yields the result by means of the Dulac criterion [14]. We use the well-known PoincaréBendixson theorem to conclude that every solution of (5.6) approaches an equilibrium.

The first statement of the theorem immediately follows from the fact that every solution of (5.6) approaches an equilibrium. If $\mathcal{R}_{0}>1$, then besides the disease free equilibrium, which is unstable according to Theorem 5.8, there exists a single locally stable endemic equilibrium $\breve{I}_{2}$. We show that no nontrivial solution can converge to the disease free equilibrium.

If $\lim _{t \rightarrow \infty} I(t)=0$ when $I(0)>0$, then it follows from $(5.6)_{2}$ that $\lim _{t \rightarrow \infty} V(t)=\frac{\phi K+\omega}{\mu+\theta+\phi}$. Then for every $\epsilon>0$ there exists a $t_{*}(\epsilon)$ such that $I(t)<\epsilon$ and $V(t)<\frac{\phi K+\omega}{\mu+\theta+\phi}+\epsilon$ for $t>t_{*}$. Using $(5.6)_{1}$ we get

$$
\begin{aligned}
\dot{I}(t) & \geq \beta\left(K-\epsilon-(1-\sigma)\left(\frac{\phi K+\omega}{\mu+\theta+\phi}+\epsilon\right)\right) I(t)-(\mu+\gamma) I(t) \\
& =\beta\left(\frac{K \cdot(\mu+\theta+\sigma \phi)}{\mu+\theta+\phi}-\frac{(1-\sigma) \omega}{\mu+\theta+\phi}\right) I(t)+(-2 \epsilon+\sigma \epsilon-(\mu+\gamma)) I(t)
\end{aligned}
$$

for $t>t_{*}$, moreover $\mathcal{R}_{0}=\frac{\beta}{\mu+\gamma}\left(\frac{K \cdot(\mu+\theta+\sigma \phi)}{\mu+\theta+\phi}-\frac{(1-\sigma) \omega}{\mu+\theta+\phi}\right)>1$ implies that there exists an $\epsilon_{1}$ small enough such that

$$
\beta\left(\frac{K \cdot(\mu+\theta+\sigma \phi)}{\mu+\theta+\phi}-\frac{(1-\sigma) \omega}{\mu+\theta+\phi}\right)+\left(-2 \epsilon_{1}+\sigma \epsilon_{1}-(\mu+\gamma)\right)>0 .
$$




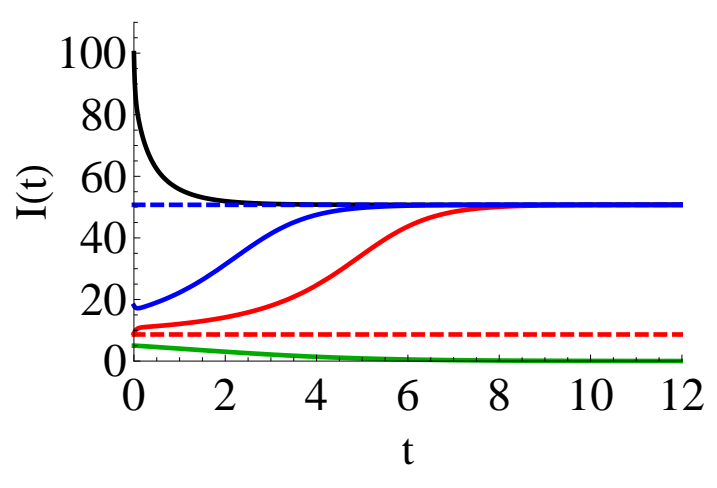

(a) Solutions of system (5.6).

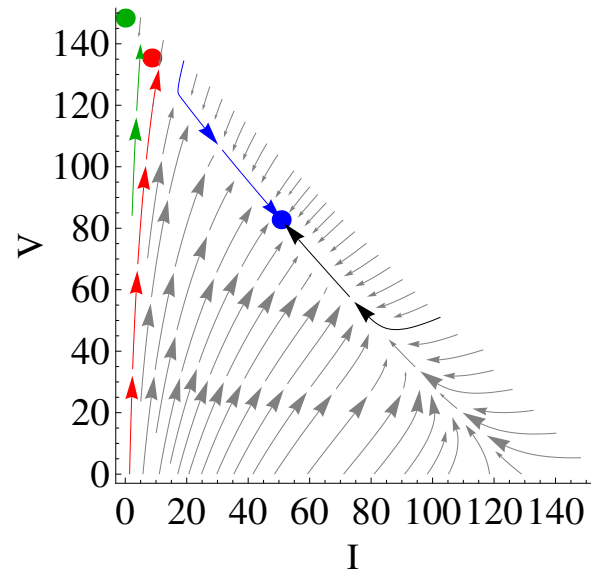

(b) Stream plot of system (5.6) on $R: 0 \leq$ $I, V, I+V \leq K$.

Figure 5.2: Solutions of system (5.6) in case there is a backward bifurcation at $\mathcal{R}_{0}=1$ and $\mathcal{R}_{c}<\mathcal{R}_{0}<1$. We let $\Lambda(x)=\frac{x}{c+d x}$ and choose parameter values as $\mu=0.1, \gamma=12$, $\theta=0.5, \sigma=0.2, \phi=16, c=1, d=1.8, \beta=0.33, \eta=5, \omega=5$, which makes $K=153.6$ and $\mathcal{R}_{0}=0.95$. Endemic equilibria $\left(\breve{I}_{1}, \breve{V}_{1}\right)=(8.6,135.4)$ and $\left(\breve{I}_{2}, \breve{V}_{2}\right)=(50.7,82.8)$ are represented as (a) red-dashed and blue-dashed lines, (b) red and blue points, respectively. On (b) the green point denotes the unique disease free equilibrium $(0,148.4)$. Solutions with initial values $(I(0), V(0))=(9,120)$ - red curve, $(18,130)$ - blue curve and $(100,50)$ - black curve converge to $\left(\breve{I}_{2}, \breve{V}_{2}\right)$, however for $(I(0), V(0))=(5,140)$ the curve of $I$ - here, green - approaches the DFE.

With the choice of $\epsilon=\epsilon_{1}$, the right-hand side of (5.18) is linear in $I(t)$ with positive multiplier, which implies that $I(t)$ increases for $t_{*}\left(\epsilon_{1}\right)>t$ and thus, cannot converge to 0 . We conclude that no solution of (5.6) with positive initial conditions converges to the disease free equilibrium, so the endemic equilibrium indeed attracts every solution.

If there is a backward bifurcation at $\mathcal{R}_{0}=1$, then besides the disease free equilibrium there exist two endemic equilibra on $\left(\mathcal{R}_{c}, 1\right)$, one locally stable and one unstable (see again Theorem 5.8). As the DFE is locally stable when $\mathcal{R}_{0}<1$, we experience bistability on $\left(\mathcal{R}_{c}, 1\right)$, which implies the third statement of the theorem.

We present Figure 5.2 to illustrate the statements of this section. The values of the model parameters were set to ensure that system (5.6) undergoes a backward bifurcation at $\mathcal{R}_{0}=1$, moreover we chose the value of $\beta$ such that there exist two endemic equilibria. The plots of the figure support our results about the long-term behavior of solutions and the local stability of equilibria; solutions starting near the unstable saddle point $\left(\breve{I}_{1}, \breve{V}_{1}\right)$ 


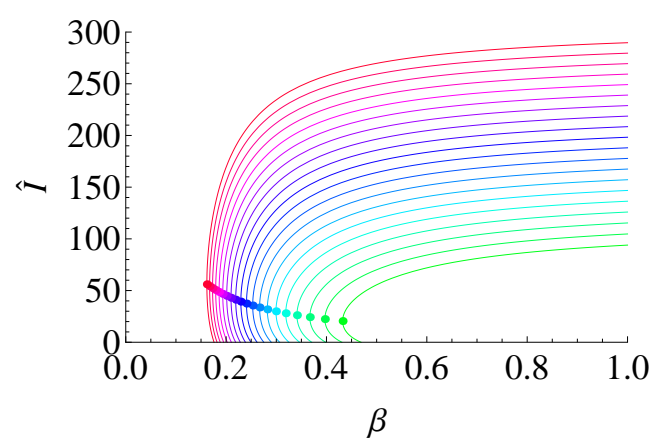

(a) $\eta=5, \omega=0,1, \ldots, 19$..

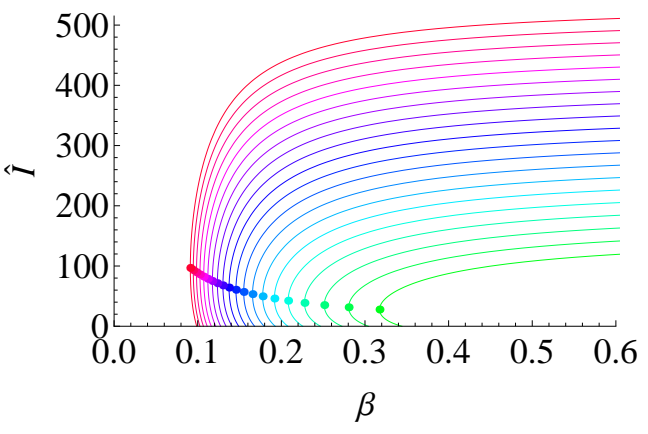

(b) $\omega=0, \eta=10,12, \ldots, 48$.

Figure 5.3: Bifurcation diagrams for 20 different values of (a) $\omega$ and (b) $\eta$ in the case when $(\theta+\mu+\sigma \phi)^{2}<\sigma(\mu+\gamma)(1-\sigma) \phi$. Proposition 5.10 implies that for all $\eta$ and $\omega$ there is a backward bifurcation at $\mathcal{R}_{0}=1$. The curves move to the left as the immigration parameter increases. We let $\Lambda(x)=\frac{x}{c+d x}$ and choose parameter values as $\mu=0.1, \gamma=12$, $\theta=0.5, \sigma=0.2, \phi=16, c=1, d=1.8$.

approach another equilibrium, however $\left(\breve{I}_{2}, \breve{V}_{2}\right)$ seems to attract every solution with $I(0)>$ $\breve{I}_{1}$ for the particular set of parameter values indicated in the caption of the figure.

\subsection{The influence of immigration on the backward bifurcation}

In this section, we would like to investigate the effect of parameters $\eta$ and $\omega$ on the bifurcation curve. In Section 5.4 we gave the condition (5.13)

$$
\frac{(1-\sigma) \omega}{K}>\frac{(\theta+\mu+\sigma \phi)^{2}-\sigma(\mu+\gamma)(1-\sigma) \phi}{(\theta+\mu+\sigma \phi)+\sigma(\mu+\gamma)}
$$

for the existence of backward bifurcation at $\mathcal{R}_{0}=1$; in what follows we analyze this inequality in terms of the immigration parameters. We keep in mind that if there is no backward bifurcation at $\mathcal{R}_{0}=1$, then there is forward bifurcation, i.e., there always exists an endemic equilibrium for $\mathcal{R}_{0}>1$.

First, we present results about how the existence of backward bifurcation depends on $\eta$ and $\omega$. The nonnegativity of $\omega$ and $K$ immediately yields the following proposition.

Proposition 5.10. If $(\theta+\mu+\sigma \phi)^{2}<\sigma(\mu+\gamma)(1-\sigma) \phi$, then for all $\eta$ and $\omega$ there is a backward bifurcation at $\mathcal{R}_{0}=1$.

The special case of $\omega=0$ automatically makes the left-hand side of inequality (5.13) zero, hence in this case there is a backward bifurcation if and only if the right-hand side is negative; note that the right-hand side is independent of $\eta$. 
Proposition 5.11. If $\omega=0$, then there is a backward bifurcation at $\mathcal{R}_{0}=1$ if and only if $(\theta+\mu+\sigma \phi)^{2}<\sigma(\mu+\gamma)(1-\sigma) \phi$. This also means that in this case $\eta$ has absolutely no effect on the direction of the bifurcation.

Figure 5.3 shows how the bifurcation curve deforms as we increase (a) $\omega$ and (b) $\eta$. Parameter values $\mu=0.1, \gamma=12, \theta=0.5, \sigma=0.2, \phi=16$ were chosen so that the condition $(\theta+\mu+\sigma \phi)^{2}<\sigma(\mu+\gamma)(1-\sigma) \phi$ holds $(14.44<30.976)$.

After all this, the following question arises naturally: is it possible to have backward bifurcation at $\mathcal{R}_{0}=1$ if $(\theta+\mu+\sigma \phi)^{2} \geq \sigma(\mu+\gamma)(1-\sigma) \phi$, i.e., if the right-hand side of condition (5.13) is nonnegative? Recall that if $\omega=0$ then $(\theta+\mu+\sigma \phi)^{2} \geq \sigma(\mu+\gamma)(1-\sigma) \phi$ means forward bifurcation.

Note that the right-hand side of (5.13) is independent of $\eta$ and $\omega$; however, $K$ depends on both of these parameters, $\mu$ and the birth function $\Lambda$. As we did not define $\Lambda$ explicitly (in Section 5.2, we only gave conditions to ensure that for each $\eta, \omega \geq 0$ the population carrying capacity $K>0$ can be defined uniquely), it is not clear how the left-hand side of (5.13) depends on the immigration parameters. In the sequel, we use the general form

$$
\Lambda(x)=\frac{x}{c+d x}
$$

for the birth function with parameters $0<c<1 / \mu$ and $d>0$; it is not hard to see that with this definition all the conditions made in Section 5.2 for $\Lambda$ are satisfied. The carrying capacity $K(\mu, \eta, \omega)$ can be obtained as the solution of

$$
\Lambda(x)=\mu x-\eta-\omega
$$

which, with our above definition (5.19), gives the second-order equation

$$
x^{2} \mu d+x(-1+c \mu-d(\eta+\omega))-c(\eta+\omega)=0 .
$$

The unique positive root yields $K$ as

$$
K(\mu, \eta, \omega)=\frac{1-c \mu+d(\eta+\omega)}{2 \mu d}+\frac{\sqrt{(1-c \mu+d(\eta+\omega))^{2}+4 \mu d c(\eta+\omega)}}{2 \mu d} .
$$

Our assumption $c<1 / \mu$ implies $1-c \mu>0$, hence

$$
\begin{aligned}
\frac{K}{\omega} & =\frac{1}{2 \mu d}\left(\frac{1-c \mu+d \eta}{\omega}+d+\sqrt{\left(\frac{1-c \mu+d \eta}{\omega}+d\right)^{2}+\frac{4 \mu d c \eta}{\omega^{2}}+\frac{4 \mu d c}{\omega}}\right) \\
& >\frac{1}{2 \mu d}\left(\frac{1-c \mu+d \eta}{\omega}+d+\frac{1-c \mu+d \eta}{\omega}+d\right) \\
& >\frac{1}{2 \mu d} 2 d=\frac{1}{\mu}
\end{aligned}
$$


and thus

$$
\frac{(1-\sigma) \omega}{K}<(1-\sigma) \mu
$$

It also follows from the above computations that $\lim _{\omega \rightarrow \infty} \frac{(1-\sigma) \omega}{K}=(1-\sigma) \mu$, i.e., although the left-hand side of $(5.13)$ is always less than $(1-\sigma) \mu$, the expression gets arbitrary close to this limit as $\omega$ approaches $\infty$.

Next we fix every model parameter but $\eta$ and $\omega$ and obtain two propositions as follows.

Proposition 5.12. Assume that $(\theta+\mu+\sigma \phi)^{2} \geq \sigma(\mu+\gamma)(1-\sigma) \phi$ holds. If the condition

$$
(\theta+\mu+\sigma \phi)(\theta+\sigma \mu+\sigma \phi)<\sigma(1-\sigma)(\mu+\gamma)(\mu+\phi)
$$

is satisfied, then for any $\eta$ there is an $\omega_{c}$ such that for any $\omega \in\left(\omega_{c}, \infty\right)$ there is a backward bifurcation at $\mathcal{R}_{0}=1$, and for any $\omega \in\left[0, \omega_{c}\right]$ there is a forward bifurcation at $\mathcal{R}_{0}=1$. In case the above condition does not hold, then for any $\eta$ and $\omega$ there is a forward bifurcation at $\mathcal{R}_{0}=1$.

Proof. If

$$
\begin{aligned}
(\theta+\mu+\sigma \phi)(\theta+\sigma \mu+\sigma \phi) & \geq \sigma(1-\sigma)(\mu+\gamma)(\mu+\phi), \\
(\theta+\mu+\sigma \phi)\left(\frac{\theta+\mu+\sigma \phi}{1-\sigma}-\mu\right) & \geq \sigma(\mu+\gamma)(\mu+\phi), \\
\frac{(\theta+\mu+\sigma \phi)^{2}}{1-\sigma}-\sigma(\mu+\gamma) \phi & \geq \mu(\theta+\mu+\sigma \phi)+\mu \sigma(\mu+\gamma)), \\
\frac{(\theta+\mu+\sigma \phi)^{2}-\sigma(\mu+\gamma)(1-\sigma) \phi}{(\theta+\mu+\sigma \phi)+\sigma(\mu+\gamma)} & \geq(1-\sigma) \mu,
\end{aligned}
$$

then it follows from (5.21) that backward bifurcation is not possible at $\mathcal{R}_{0}=1$, since the right-hand side of condition (5.13) is always greater than or equal to the left-hand side. Next let us consider the case when

$$
\begin{aligned}
(\theta+\mu+\sigma \phi)(\theta+\sigma \mu+\sigma \phi) & <\sigma(1-\sigma)(\mu+\gamma)(\mu+\phi), \\
\frac{(\theta+\mu+\sigma \phi)^{2}-\sigma(\mu+\gamma)(1-\sigma) \phi}{(\theta+\mu+\sigma \phi)+\sigma(\mu+\gamma)} & <(1-\sigma) \mu .
\end{aligned}
$$

We show that $\frac{(1-\sigma) \omega}{K}$ is monotone increasing in $\omega$; if so, then, following relation (5.21) and the discussion afterwards, the formulas $\frac{(1-\sigma) \cdot 0}{K(\mu, \eta, 0)}=0$ and $\lim _{\omega \rightarrow \infty} \frac{(1-\sigma) \omega}{K(\mu, \eta, \omega)}=(1-\sigma) \mu$ imply that $\omega_{c}$ can be defined uniquely by

$$
\frac{(1-\sigma) \omega_{c}}{K\left(\mu, \eta, \omega_{c}\right)}=\frac{(\theta+\mu+\sigma \phi)^{2}-\sigma(\mu+\gamma)(1-\sigma) \phi}{(\theta+\mu+\sigma \phi)+\sigma(\mu+\gamma)}
$$

and from the monotonicity it follows that the condition for the backward bifurcation (5.13) is satisfied if and only if $\omega>\omega_{c}$.

We obtain the derivative

$$
\frac{\partial}{\partial \omega}\left(\frac{\omega}{K}\right)=\frac{K-\omega \frac{\partial K}{\partial \omega}}{K^{2}}
$$


which implies that $\frac{(1-\sigma) \omega}{K}$ increases in $\omega$ if and only if $K-\omega \frac{\partial K}{\partial \omega}$ is positive. With our assumption $1-c \mu>0$, the computations

$$
\begin{aligned}
K-\omega \frac{\partial K}{\partial \omega}= & \frac{1-c \mu+d(\eta+\omega)+\sqrt{(1-c \mu+d(\eta+\omega))^{2}+4 \mu d c(\eta+\omega)}}{2 \mu d} \\
& -\omega d \frac{1}{2 \mu d}\left(1+\frac{1-c \mu+d(\eta+\omega)+2 \mu c}{\sqrt{(1-c \mu+d(\eta+\omega))^{2}+4 \mu d c(\eta+\omega)}}\right) \\
= & \frac{1-c \mu+d \eta}{2 \mu d}+\frac{(1-c \mu+d(\eta+\omega))^{2}+4 \mu d c(\eta+\omega)}{2 \mu d \sqrt{(1-c \mu+d(\eta+\omega))^{2}+4 \mu d c(\eta+\omega)}} \\
& -\frac{\omega d(1-c \mu+d(\eta+\omega)+2 \mu c)}{2 \mu d \sqrt{(1-c \mu+d(\eta+\omega))^{2}+4 \mu d c(\eta+\omega)}} \\
= & \frac{1-c \mu+d \eta}{2 \mu d}+\frac{(1-c \mu+d(\eta+\omega))(1-c \mu+d \eta)+4 \mu d c \eta+2 \mu d c \omega}{2 \mu d \sqrt{(1-c \mu+d(\eta+\omega))^{2}+4 \mu d c(\eta+\omega)}}>0
\end{aligned}
$$

yield the result.

With other words, for parameter values satisfying the assumption and condition of Proposition 5.12, a unique critical value $\omega_{c}$ can be defined which works as a threshold of $\omega$ for the backward bifurcation: there is no backward bifurcation if $\omega \leq \omega_{c}$, and once $\omega$ is large enough so that a backward bifurcation is established at $\mathcal{R}_{0}=1$, it can not happen that for any larger values of $\omega$ the system undergoes forward bifurcation again. With certain conditions, such threshold also exists for $\eta$ as we show it in the following proposition.

Proposition 5.13. Assume that $(\theta+\mu+\sigma \phi)^{2} \geq \sigma(\mu+\gamma)(1-\sigma) \phi$ holds, and fix $\omega$. If $\omega$ is such that

$$
\frac{(1-\sigma) \omega}{K(\mu, 0, \omega)}>\frac{(\theta+\mu+\sigma \phi)^{2}-\sigma(\mu+\gamma)(1-\sigma) \phi}{(\theta+\mu+\sigma \phi)+\sigma(\mu+\gamma)},
$$

then there exists $\eta_{c}>0$ such that there is a backward bifurcation at $\mathcal{R}_{0}=1$ for $\eta<\eta_{c}$, and the system undergoes a forward bifurcation for $\eta \geq \eta_{c}$. If the above inequality does not hold then there is a forward bifurcation at $\mathcal{R}_{0}=1$.

Proof. First we note that $K(\mu, \eta, \omega)$ (defined in (5.20)) is an increasing function of $\eta$ and it attains its minimum at $\eta=0$. This implies that

$$
\frac{(1-\sigma) \omega}{K(\mu, \eta, \omega)} \leq \frac{(1-\sigma) \omega}{K(\mu, 0, \omega)}
$$

for all $\eta$, hence the condition for the backward bifurcation (5.13) cannot be satisfied if

$$
\frac{(1-\sigma) \omega}{K(\mu, 0, \omega)} \leq \frac{(\theta+\mu+\sigma \phi)^{2}-\sigma(\mu+\gamma)(1-\sigma) \phi}{(\theta+\mu+\sigma \phi)+\sigma(\mu+\gamma)}
$$




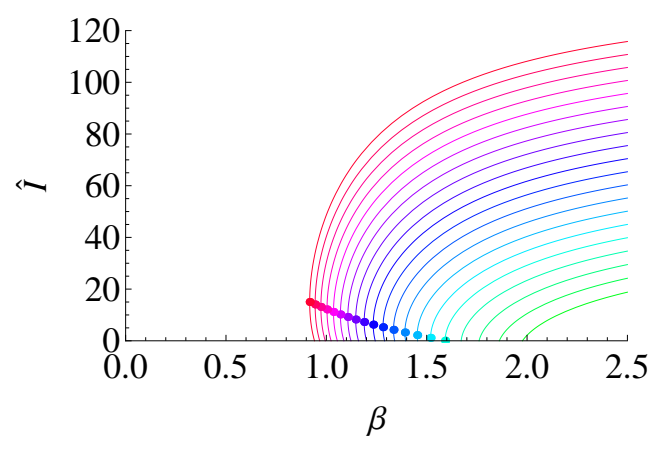

(a) $\eta=10, \omega=1,6, \ldots, 96$.

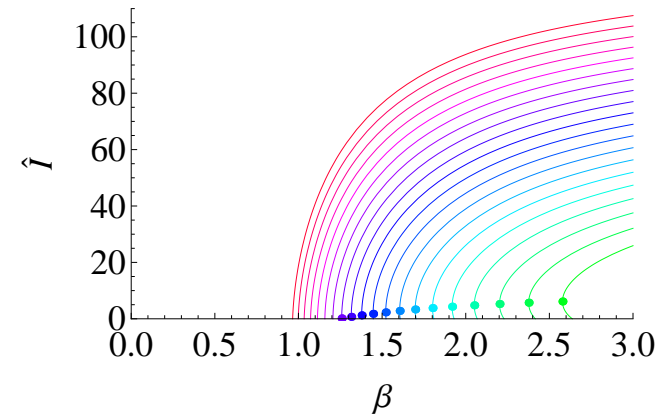

(b) $\omega=60, \eta=1,6, \ldots, 96$.

Figure 5.4: Bifurcation diagrams for 20 different values of (a) $\omega$ and (b) $\eta$ in the case when $(\theta+\mu+\sigma \phi)^{2} \geq \sigma(\mu+\gamma)(1-\sigma) \phi$. The curves move to the left as the immigration parameter increases. We let $\Lambda(x)=\frac{x}{c+d x}$ and choose parameter values as (a) $\mu=1, \gamma=7.5, \theta=0.5$, $\sigma=0.02, \phi=16, c=0.1, d=0.03$, (b) $\mu=1.5, \gamma=11, \theta=0.5, \sigma=0.02, \phi=16$, $c=1 / 15, d=9 / 300$.

On the other hand, $K(\mu, \eta, \omega)$ takes arbitrary large values, and hence $\frac{(1-\sigma) \omega}{K(\mu, \eta, \omega)}$ converges to zero monotonically as $\eta$ increases, so if

$$
\frac{(1-\sigma) \omega}{K(\mu, 0, \omega)}>\frac{(\theta+\mu+\sigma \phi)^{2}-\sigma(\mu+\gamma)(1-\sigma) \phi}{(\theta+\mu+\sigma \phi)+\sigma(\mu+\gamma)},
$$

then there is a unique $\eta_{c}>0$ which satisfies

$$
\frac{(1-\sigma) \omega}{K\left(\mu, \eta_{c}, \omega\right)}=\frac{(\theta+\mu+\sigma \phi)^{2}-\sigma(\mu+\gamma)(1-\sigma) \phi}{(\theta+\mu+\sigma \phi)+\sigma(\mu+\gamma)}
$$

and the monotonicity of $K$ in $\eta$ yields that for $\eta<\eta_{c}\left(\eta \geq \eta_{c}\right)$ the condition for the backward bifurcation (5.13) holds (does not hold). Thus it is clear that $\eta_{c}$ is a threshold for the existence of backward bifurcation. Note that if $(\theta+\mu+\sigma \phi)^{2}=\sigma(\mu+\gamma)(1-\sigma) \phi$ then $\eta_{c}=\infty$, i.e., for each value of $\eta$ there is a backward bifurcation if $\omega>0$. The proof is complete.

We illustrate Propositions 5.12 and 5.13 with Figure 5.4. With parameter values $\mu=1$, $\gamma=7.5, \theta=0.5, \sigma=0.02, \phi=16, c=0.1, d=0.03$ and $\eta=10$ used for Figure 5.4 (a), the condition in Proposition 5.12 becomes $1.5288<2.8322$. In case of Figure 5.4 (b), the parameters $\mu=1.5, \gamma=11, \theta=0.5, \sigma=0.02, \phi=16, c=1 / 15, d=9 / 300$ and $\omega=60$ give $\frac{(1-\sigma) \omega}{K(\mu, 0, \omega)}=0.956928$ and $\frac{(\theta+\mu+\sigma \phi)^{2}-\sigma(\mu+\gamma)(1-\sigma) \phi}{(\theta+\mu+\sigma \phi)+\sigma(\mu+\gamma)}=0.569027$, so the condition in Proposition 5.13 is satisfied. It is easy to check that the assumption $(\theta+\mu+\sigma \phi)^{2} \geq \sigma(\mu+\gamma)(1-\sigma) \phi$ holds in both cases since (a) $3.3124 \geq 2.6656$ and (b) $5.3824 \geq 3.92$.

Proposition 5.10 states that for any values of $\eta$ and $\omega$ the condition $(\theta+\mu+\sigma \phi)^{2}<$ $\sigma(\mu+\gamma)(1-\sigma) \phi$ is sufficient for the existence of a backward bifurcation at $\mathcal{R}_{0}=1$; 
moreover we know from Proposition 5.11 that it is also necessary in the special case of $\omega=0$. We remark that backward bifurcation is possible for any $\eta \geq 0$ and $\omega>0$, even if $(\theta+\mu+\sigma \phi)^{2} \geq \sigma(\mu+\gamma)(1-\sigma) \phi$. Let us choose $\eta \geq 0$ and $\omega>0$ arbitrary, fix parameters $\mu, \sigma, \phi$, and choose $\theta$ and $\gamma$ such that $(\theta+\mu+\sigma \phi)^{2}=\sigma(\mu+\gamma)(1-\sigma) \phi$ holds. As now the right-hand side of condition (5.13) is 0 and $\omega, K>0$, there is a backward bifurcation, moreover it is easy to see that the right-hand side is increasing in $\theta$. Thus, due to the continuous dependence of the right-hand side on $\theta$, there is an interval for $\theta$ (with all the other parameters fixed) where condition (5.13) still holds, though $(\theta+\mu+\sigma \phi)^{2}>\sigma(\mu+\gamma)(1-\sigma) \phi$, since the quadratic term increases in $\theta$.

Next, we investigate how immigration deforms the bifurcation curve. Let us denote by $\beta_{0}$ the value of the transmission rate for which $\mathcal{R}_{0}=1$ is satisfied, using (5.8) it can be obtained as

$$
\beta_{0}=\frac{(\mu+\theta+\phi)(\mu+\gamma)}{K \cdot(\mu+\theta+\sigma \phi)-(1-\sigma) \omega} .
$$

Proposition 5.14. It holds that $\beta_{0}$ decreases in both $\omega$ and $\eta$.

Proof. Using (5.22), we see that $\beta_{0}$ decreases as $\eta$ increases since

$$
\frac{\partial}{\partial \eta}(K \cdot(\mu+\theta+\sigma \phi)-(1-\sigma) \omega)=\frac{\partial K}{\partial \eta} \cdot(\mu+\theta+\sigma \phi)>0 .
$$

On the other hand, $\beta_{0}$ decreases in $\omega$ if and only if

$$
\frac{\partial}{\partial \omega}(K \cdot(\mu+\theta+\sigma \phi)-(1-\sigma) \omega)=\frac{\partial K}{\partial \omega} \cdot(\mu+\theta+\sigma \phi)-(1-\sigma)>0 .
$$

First, $\frac{\partial K}{\partial \omega}>\frac{1}{\mu}$ since

$$
\begin{array}{r}
\frac{1-c \mu+d(\eta+\omega)+2 \mu c}{\sqrt{(1-c \mu+d(\eta+\omega))^{2}+4 \mu d c(\eta+\omega)}}>1, \\
\frac{\partial K}{\partial \omega}=\frac{1}{2 \mu}\left(1+\frac{1-c \mu+d(\eta+\omega)+2 \mu c}{\sqrt{(1-c \mu+d(\eta+\omega))^{2}+4 \mu d c(\eta+\omega)}}\right)>\frac{1}{\mu},
\end{array}
$$

second, from

$$
\begin{gathered}
\theta+\sigma \phi>-\mu \sigma, \\
\mu+\theta+\sigma \phi>\mu(1-\sigma),
\end{gathered}
$$

we have $\frac{1}{\mu}>\frac{1-\sigma}{\mu+\theta+\sigma \phi}$. We conclude that

$$
\frac{\partial K}{\partial \omega}>\frac{1}{\mu}>\frac{1-\sigma}{\mu+\theta+\sigma \phi}
$$

and hence $\beta_{0}$ decreases as $\omega$ increases. 
We recall that endemic equilibria $\breve{I}_{1}$ and $\breve{I}_{2}$ were defined as

$$
\breve{I}_{1}=\frac{-B-\sqrt{B^{2}-4 A C}}{2 A}, \quad \breve{I}_{2}=\frac{-B+\sqrt{B^{2}-4 A C}}{2 A},
$$

with $A, B$ and $C$ given in (5.12). Obviously $-B-\sqrt{B^{2}-4 A C}>0$ where $\breve{I}_{1}$ exists and $-B+\sqrt{B^{2}-4 A C}>0$ where $\breve{I}_{2}$ exists.

Proposition 5.15. For the endemic equilibrium $\breve{I}_{2}$ it holds that $\frac{\partial}{\partial \omega} \breve{I}_{2}, \frac{\partial}{\partial \eta} \breve{I}_{2}>0$, and the inequalities $\frac{\partial}{\partial \omega} \breve{I}_{1}, \frac{\partial}{\partial \eta} \breve{I}_{1}<0$ are satisfied for the endemic equilibrium $\breve{I}_{1}$. The equilibrium $\breve{I}_{1}=\breve{I}_{2}=\frac{-B}{2 A}$ increases in both $\omega$ and $\eta$.

Proof. Since

$$
\begin{aligned}
& \frac{\partial A C}{\partial \omega}=-\sigma \beta(\mu+\theta+\sigma \phi) \frac{\partial K}{\partial \omega}+\sigma \beta(1-\sigma), \\
& \frac{\partial A C}{\partial \eta}=-\sigma \beta(\mu+\theta+\sigma \phi) \frac{\partial K}{\partial \eta},
\end{aligned}
$$

we derive

$$
\begin{aligned}
\frac{\partial}{\partial \omega}\left(\sqrt{B^{2}-4 A C}-B\right) & =\frac{2 B \frac{\partial B}{\partial \omega}-4\left(-\sigma \beta(\mu+\theta+\sigma \phi) \frac{\partial K}{\partial \omega}+\sigma \beta(1-\sigma)\right)}{2 \sqrt{B^{2}-4 A C}}-\frac{\partial B}{\partial \omega} \\
& =\frac{\frac{\partial B}{\partial \omega}\left(B-\sqrt{B^{2}-4 A C}\right)}{\sqrt{B^{2}-4 A C}}+\frac{2 \sigma \beta\left((\mu+\theta+\sigma \phi) \frac{\partial K}{\partial \omega}-(1-\sigma)\right)}{\sqrt{B^{2}-4 A C}} \\
\frac{\partial}{\partial \eta}\left(\sqrt{B^{2}-4 A C}-B\right) & =\frac{2 B \frac{\partial B}{\partial \eta}-4\left(-\sigma \beta(\mu+\theta+\sigma \phi) \frac{\partial K}{\partial \omega}\right)}{2 \sqrt{B^{2}-4 A C}}-\frac{\partial B}{\partial \eta} \\
& =\frac{\frac{\partial B}{\partial \eta}\left(B-\sqrt{B^{2}-4 A C}\right)}{\sqrt{B^{2}-4 A C}}+\frac{2 \sigma \beta(\mu+\theta+\sigma \phi) \frac{\partial K}{\partial \eta}}{\sqrt{B^{2}-4 A C}}
\end{aligned}
$$

moreover it follows from (5.23), $\frac{\partial B}{\partial \omega}=-\sigma \beta \frac{\partial K}{\partial \omega}<0, \frac{\partial B}{\partial \eta}=-\sigma \beta \frac{\partial K}{\partial \eta}<0$ and $B-$ $\sqrt{B^{2}-4 A C}<0$ that

$$
\begin{aligned}
& \frac{\partial}{\partial \omega}\left(\sqrt{B^{2}-4 A C}-B\right)>0, \\
& \frac{\partial}{\partial \eta}\left(\sqrt{B^{2}-4 A C}-B\right)>0 .
\end{aligned}
$$

Similarly, using $B+\sqrt{B^{2}-4 A C}<0$ we get

$$
\begin{aligned}
\frac{\partial}{\partial \omega}\left(\sqrt{B^{2}-4 A C}+B\right) & =\frac{\frac{\partial B}{\partial \omega}\left(B+\sqrt{B^{2}-4 A C}\right)}{\sqrt{B^{2}-4 A C}}+\frac{2 \sigma \beta\left((\mu+\theta+\sigma \phi) \frac{\partial K}{\partial \omega}-(1-\sigma)\right)}{\sqrt{B^{2}-4 A C}}>0, \\
\frac{\partial}{\partial \eta}\left(\sqrt{B^{2}-4 A C}+B\right) & =\frac{\frac{\partial B}{\partial \eta}\left(B+\sqrt{B^{2}-4 A C}\right)}{\sqrt{B^{2}-4 A C}}+\frac{2 \sigma \beta(\mu+\theta+\sigma \phi) \frac{\partial K}{\partial \eta}}{\sqrt{B^{2}-4 A C}}>0 .
\end{aligned}
$$

We conclude that

$$
\begin{aligned}
\frac{\partial}{\partial \omega} \breve{I}_{1} & =\frac{-\frac{\partial}{\partial \omega}\left(\sqrt{B^{2}-4 A C}+B\right)}{2 A}<0, \\
\frac{\partial}{\partial \eta} \breve{I}_{1} & =\frac{-\frac{\partial}{\partial \eta}\left(\sqrt{B^{2}-4 A C}+B\right)}{2 A}<0,
\end{aligned}
$$


furthermore

$$
\begin{gathered}
\frac{\partial}{\partial \omega} \breve{I}_{2}=\frac{\frac{\partial}{\partial \omega}\left(\sqrt{B^{2}-4 A C}-B\right)}{2 A}>0, \\
\frac{\partial}{\partial \eta} \breve{I}_{2}=\frac{\frac{\partial}{\partial \eta}\left(\sqrt{B^{2}-4 A C}-B\right)}{2 A}>0
\end{gathered}
$$

this is, the equilibrium $\breve{I}_{1}=\breve{I}_{2}=\frac{-B}{2 A}$ is increasing in both $\omega$ and $\eta$ since $A$ is independent of these parameters and $\frac{\partial B}{\partial \omega}<0, \frac{\partial B}{\partial \eta}<0$.

These results give us information about how the bifurcation curve changes when the immigration parameters increase. If there is a forward bifurcation at $\mathcal{R}_{0}=1$, the curve moves to the left since $\beta_{0}$ decreases in $\eta$ and $\omega$, and the curve expands because $\frac{\partial}{\partial \omega} \breve{I}_{2}, \frac{\partial}{\partial \eta} \breve{I}_{2}>$ 0 . In case there is a backward bifurcation at $\mathcal{R}_{0}=1, \beta_{0}$ again moves to the left, and $\frac{\partial}{\partial \omega} \breve{I}_{1}, \frac{\partial}{\partial \eta} \breve{I}_{1}<0$ and $\frac{\partial}{\partial \omega} \breve{I}_{2}, \frac{\partial}{\partial \eta} \breve{I}_{2}>0$ imply that for each fixed $\beta$ the two equilibria move away from each other in the region where they coexist, moreover $\breve{I}_{2}$ increases when it is the only endemic equilibrium. The singular point of the bifurcation curve, where the equilibrium is $-B / 2 A$, moves upward as $\eta$ and $\omega$ increase, this together with the above described behavior of $\breve{I}_{1}$ and $\breve{I}_{2}$ imply that the left-most equilibrium cannot move to the right, or equivalently, the corresponding value of the transmission rate $\beta_{c}$ decreases if we increase $\eta$ and $\omega$. We give the last statement of the above discussion in the form of a proposition. See Figures 5.3 and 5.4 for visual proof of the results of this section.

Proposition 5.16. In case there is a backward bifurcation at $\mathcal{R}_{0}=1, \beta_{c}$ decreases in both $\omega$ and $\eta$.

Actually, using (5.22), it is easy to see that $\beta_{0}$ converges to 0 as any of the immigration parameters approaches infinity: for any fixed $\omega(\eta)$, the carrying capacity $K$ reaches arbitrary large values if we increase $\eta(\omega)$, moreover $\mu K-\omega$ is positive by assumption, hence

$$
\begin{aligned}
\lim _{\omega \rightarrow \infty} & (K(\mu+\theta+\sigma \phi)-(1-\sigma) \omega) \\
& =\lim _{\omega \rightarrow \infty}(K(\theta+\sigma \phi)+\sigma \omega+\mu K-\omega)=\infty .
\end{aligned}
$$

The inequality $\beta_{c}<\beta_{0}$ implies that $\beta_{c}$ also goes to 0 as $\omega \rightarrow \infty$ or $\eta \rightarrow \infty$. We can also show that in the special case of $\omega=0$, increasing $\eta$ decreases the region where two endemic equilibria exist. The equation (5.17) for $\beta_{c}$ then reformulates as

$$
\begin{aligned}
\beta_{c} K \sigma & =x-U+2 \sqrt{U W} \\
& =\sigma(\mu+\gamma)-(\theta+\mu+\sigma \phi)+2 \sqrt{-(\theta+\mu+\sigma \phi) \sigma(\mu+\gamma)+\sigma(\gamma+\mu)(\mu+\phi+\theta)} \\
& =\sigma(\mu+\gamma)-(\theta+\mu+\sigma \phi)+2 \sqrt{\sigma(\mu+\gamma) \phi(1-\sigma)},
\end{aligned}
$$

thus for $\beta_{0}-\beta_{c}$ we have

$$
\left(\beta_{0}-\beta_{c}\right) K \sigma=\frac{\sigma(\mu+\theta+\phi)(\mu+\gamma)}{(\mu+\theta+\sigma \phi)}-\sigma(\mu+\gamma-((\theta+\mu+\sigma \phi)+2 \sqrt{\sigma(\mu+\gamma) \phi(1-\sigma)}) .
$$


The right-hand side is independent of $\eta$ and $K$ increases monotonically as $\eta$ increases, so the length of the interval $\left(\beta_{c}, \beta_{0}\right)$ decreases as $\eta$ increases.

In the light of the results of this section we conclude that, although SIVS models without immigration can also exhibit backward bifurcation [7], incorporating the possibility of the inflow of non-infectives may significantly influence the dynamics: under certain conditions on the model parameters, increasing $\omega$, just as decreasing $\eta$, can drive a system with forward bifurcation into backward bifurcation and the existence of multiple endemic equilibria. Furthermore, we showed that including immigration moves the left-most point of the bifurcation curve to the left, which means that the larger the values of the immigration parameters the smaller the threshold for the emergence of endemic equilibria.

\subsection{Revisiting the three-dimensional system}

Based on our results for system (5.6), we draw some conclusions on the global behavior of the original model (5.4). Given that $N(t)$ converges, and substituting $S(t)=N(t)-$ $I(t)-V(t),(5.4)_{2}$ and $(5.4)_{3}$ together can be considered as an asymptotically autonomous system with limiting system (5.6). We use the theory from [44].

Theorem 5.17. All nonnegative solutions of (5.4) converge to an equilibrium. In particular, if $\mathcal{R}_{0}>1$, then the endemic equilibrium is globally asymptotically stable. If there is a forward bifurcation for (5.6) and $\mathcal{R}_{0} \leq 1$, or there is a backward bifurcation for (5.6) and $\mathcal{R}_{0}<\mathcal{R}_{c}$, then the disease free equilibrium is globally asymptotically stable.

Proof. Theorem 5.9 excluded periodic orbits in the limit system by a Dulac function, hence we can apply Corollary 2.2. of [44] and conclude that all solutions of $(5.4)_{2}-(5.4)_{3}$ converge. As $I(t), V(t)$ and $N(t)$ converge, $S(t)$ converges as well for system (5.4).

Now consider the case $\mathcal{R}_{0}>1$. Then the endemic equilibrium is globally asymptotically stable for (5.4) (see Theorem 5.9), and its basin of attraction is the whole phase space except the disease free equilibrium. We can proceed analogously as in (5.18) to show that no positive solutions of $(5.4)_{2}-(5.4)_{3}$ can converge to $(0, \bar{V})$ when $\mathcal{R}_{0}>1$, since $N(t)>K-\epsilon$ holds for sufficiently large $t$. Thus, the $\omega$-limit set of any positive solution of $(5.4)_{2}-(5.4)_{3}$ intersects the basin of attraction of the endemic equilibrium in the limit system, and then by Theorem 2.3 of [44] we conclude that the positive solutions of $(5.4)_{2}-(5.4)_{3}$ converge to the endemic equilibrium.

When the disease free is the unique equilibrium of (5.6), (i.e., when $\mathcal{R}_{0} \leq 1$ in the case of forward, or $\mathcal{R}_{0}<\mathcal{R}_{c}$ in the case of backward bifurcation), then it is globally 
asymptotically stable for (5.6) (see Theorem 5.9) with the basin of attraction being the whole space, thus Theorem 2.3 of [44] ensures that the DFE is globally asymptotically stable for $(5.4)_{2}-(5.4)_{3}$ as well.

\subsection{Conclusion}

We have examined a dynamic model which describes the spread of an infectious disease in a population divided into the classes of susceptible, infected and vaccinated individuals, and took the possibility of immigration of non-infectives into account. Such an assumption is reasonable if there is an entry screening of infected individuals, or if the disease is so severe that it inhibits traveling. After obtaining some fundamental, but biologically relevant properties of the model, we investigated the possible equilibria and gave an explicit condition for the existence of backward bifurcation at $\mathcal{R}_{0}=1$ in terms of the model parameters. Our analysis showed that besides the disease free equilibrium - which always exists - there is a unique positive fixed point for $\mathcal{R}_{0}>1$, moreover in case of a backward bifurcation there exist two endemic equilibria on an interval to the left of $\mathcal{R}_{0}=1$. An equilibrium is locally asymptotically stable if and only if it corresponds to a point on the bifurcation curve where the curve is increasing, moreover it is also globally attracting if $\mathcal{R}_{0}>1$.

We investigated how the structure of the bifurcation curve depends on $\eta$ and $\omega$ (the immigration parameter for susceptible and vaccinated individuals, respectively), when other model parameters are fixed. As discussed in Propositions 5.10 and 5.12, two regions can be characterized in the parameter space where for any values of the immigration parameters, the system experiences a backward or forward bifurcation, respectively. However, under certain conditions described in Propositions 5.12 and 5.13, modifying the value of $\omega$ and $\eta$ has a significant effect on the dynamics: critical values $\omega_{c}$ and $\eta_{c}$ can be defined such that the bifurcation behavior at $\mathcal{R}_{0}=1$ changes from forward to backward when we increase $\omega$ through $\omega_{c}$ and/or we decrease $\eta$ through $\eta_{c}$. On the other hand, Propositions 5.11 and 5.13 yield that in some cases $\omega$ can be chosen so that, independently from the value of $\eta$, backward bifurcation is impossible.

We also showed that immigration decreases the value of the transmission rate for which endemic equilibria emerge, furthermore increasing $\omega$ and/or $\eta$ moves the branches of the bifurcation curve apart which implies that the stability region of the disease free equilibrium shrinks (see Figures 5.3 and 5.4). Last, we wish to point out that, as it follows from the 
discussion after Proposition 5.13, backward bifurcation is possible for any values of $\omega$ and $\eta$, so when one's aim is to mitigate the severity of an outbreak it is desirable to control the values of other model parameters, for example, the vaccination rate in a way that the scenario of backward bifurcation is never realized. 


\section{Summary}

In this Ph.D. dissertation we study the general form of nonautonomous functional differential equations where the delayed feedback function cannot be given explicitly by the system variables, but it is defined via the solution of another system of differential equations. In Chapter 2, we formulate the initial value problem as

$$
\begin{aligned}
x^{\prime}(t) & =\mathcal{F}\left(t, x_{t}\right)=f(t, x(t))+W(t, x(t-\tau)), \\
x_{\sigma} & =\varphi,
\end{aligned}
$$

where $t, \sigma \in \mathbb{R}$ with $t \geq \sigma, x: \mathbb{R} \rightarrow \mathbb{R}^{n}, f: \mathbb{R} \times \mathbb{R}^{n} \rightarrow \mathbb{R}^{n}$ and $W: \mathbb{R} \times \mathbb{R}^{n} \rightarrow \mathbb{R}^{n}$ for $n \in \mathbb{Z}_{+}$. For a fixed positive $\tau$, we define the phase space $C=C\left([-\tau, 0], \mathbb{R}^{n}\right)$ as the Banach space of continuous functions from $[-\tau, 0]$ to $\mathbb{R}^{n}$, equipped with the supremum norm. The segment $x_{t} \in C$ of the solution is defined by the relation $x_{t}(\theta)=x(t-\theta)$ for $\theta \in[-\tau, 0]$, so $\mathcal{F}: \mathbb{R} \times C \rightarrow \mathbb{R}^{n}$. Moreover, $\varphi \in C$ gives the state of the system at initial time $\sigma$. In this work, a Lipschitz condition is formulated for any function $F: \mathbb{R} \times \mathbb{R}^{j} \rightarrow \mathbb{R}^{l}, j, l \in \mathbb{Z}_{+}$, on each bounded subset of $\mathbb{R} \times \mathbb{R}^{j}$, as follows:

(Lip) For all $a, b \in \mathbb{R}$ and $M>0$, there is a $K(a, b, M)>0$ such that:

$$
\left|F\left(t, x_{1}\right)-F\left(t, x_{2}\right)\right|_{l} \leq K\left|x_{1}-x_{2}\right|_{j}, a \leq t \leq b,\left|x_{1}\right|_{j},\left|x_{2}\right|_{j} \leq M
$$

We assume that the function $f$ is continuous and satisfies (Lip) on each bounded subset of $\mathbb{R} \times \mathbb{R}^{n}$. Furthermore, an initial value problem for systems of nonautonomous ordinary differential equations is formulated as

$$
\begin{aligned}
& y^{\prime}(s)=g(s, y(s)), \\
& y\left(s_{0}\right)=y_{*},
\end{aligned}
$$

where $m \in \mathbb{Z}_{+}$, and $s_{0} \in \mathbb{R}$ and $y_{*} \in \mathbb{R}$ represent the initial time and the initial value, respectively. Here $s \in \mathbb{R}, s \geq s_{0}$ and $y: \mathbb{R} \rightarrow \mathbb{R}^{m}$, moreover the function $g: \mathbb{R} \times \mathbb{R}^{m} \rightarrow \mathbb{R}^{m}$ is continuous on $\mathbb{R} \times \mathbb{R}^{m}$ and satisfies the Lipschitz condition (Lip) on each bounded subset of $\mathbb{R} \times \mathbb{R}^{m}$. Standard arguments from the theory of ordinary differential equations guarantee that system (2.2) has a unique solution, which we denote by $y\left(s ; s_{0}, y_{*}\right)$, on the interval $\left[s_{0}, s_{0}+\alpha\right]$ for some positive $\alpha$. We make the additional assumption that: 
$(\star)$ For every $s_{0}$ and $y_{*}$, the solution $y\left(s ; s_{0}, y_{*}\right)$ of $(2.2)$ exists for $\tau$ units of time, i.e., on $\left[s_{0}, s_{0}+\tau\right]$.

We introduce two functions $h: \mathbb{R} \times \mathbb{R}^{n} \rightarrow \mathbb{R}^{m}$ and $k: \mathbb{R} \times \mathbb{R}^{m} \rightarrow \mathbb{R}^{n}$, both are continuous and satisfy (Lip) on the corresponding sets by assumption. For simplicity, we use the notation $y_{s_{0}, v}(s)=y\left(s ; s_{0}, h\left(s_{0}, v\right)\right)$ for the unique solution of $(2.2)$ in the case $y_{*}=h\left(s_{0}, v\right), v \in \mathbb{R}^{n}$. Last, the delayed feedback function $W: \mathbb{R} \times \mathbb{R}^{n} \rightarrow \mathbb{R}^{n}$ is defined as

$$
W(s, v)=k\left(s, y_{s-\tau, v}(s)\right)=k(s, y(s ; s-\tau, h(s-\tau, v))) .
$$

From this formulation it follows that $W$ is determined via the dynamics of another system of differential equations, given by (2.2). Henceforth, we refer to (2.1) as the general form of functional differential equations with dynamically defined delayed term.

For the solution of system (2.1), we obtain the usual existence, uniqueness and continuous dependence results by showing a Lipschitz condition for $\mathcal{F}$. As it is illustrated in the thesis by various biological applications, functional differential equations with dynamically defined delayed feedback function may arise from the mathematical analysis of dynamic models in life sciences. Thus, it is natural to consider some biologically relevant properties of the initial value problem, like the nonnegativity of solutions or the stability of equilibria of the autonomous system.

Functional differential equations have many applications in biological sciences. In the dissertation, we propose some models from population dynamics and epidemiology, where the delay terms in the model equations depend on the solution of another dynamical system. First, a simple model for the growth of a single population with fixed period of temporary separation is presented in Chapter 1. Then we investigate a class of delayed disease transmission models in Chapters 3 and 4 to describe the propagation of epidemics on transportation networks. Recent pandemics, like the 2002-2003 SARS epidemic or the 2009 A(H1N1) influenza outbreak illustrated the role of commercial aviation in the spatial spread of infectious diseases. Based on the consideration that these diseases progress so fast that any short delay might be significant, we incorporate into the model the time needed to complete transportation between the regions, and use a delay differential system to describe the spread of infection in the regions. Furthermore, we account for the fact that some communicable diseases, such as tuberculosis, measles and influenza, have been known to be transmissible during commercial flights, hence in the model formulation we also consider the possible infections during travel. It follows that the inflow of individuals into a region upon completing a trip arises as a delayed feedback term in the model equations, moreover this term is determined by another system which describes the disease 
dynamics during travel.

We introduce an SEAIR (susceptible-exposed-asymptomatic infected-infectedrecovered)-based model in Chapter 3 to investigate the spread of an epidemic in two regions which are connected by transportation. Disease behavior during travel is modeled by an age-structured system, where age is the time elapsed since the start of the travel. Our model is in contrast to many existing epidemic models which assume that the speed of disease spread between regions decreases as the distance between those regions increases. These models are called "gravity-type" models, where the expression reflects the inverse relationship of the speed of disease spread to distance. On the other hand, in our model we take into consideration that the transmission rate of an infectious disease can be much higher than usual when a large number of passengers are sharing the same cabin during long distance travel. This implies that the longer the flight between two regions, the more infections are expected. Thus, our model is of "antigravity-type" in principle. We distinguish local residents from visitors in the model setup to account for differences in mixing patterns and travel behaviors. After showing that the model is equivalent to a twenty-dimensional system of autonomous differential equations of the form of (2.1), we apply the results of Chapter 2 to our model. Then we present a mathematical procedure for the calculation of the basic reproduction number. This method also allows us to obtain stability results for the model by making use of the theory of cooperative and irreducible systems for delay differential equations and ordinary differential equations. Our approach of obtaining $\mathcal{R}_{0}$ is particularly designed for models with the delayed term determined via the solution of another differential system. We parametrize our model for influenza and use real demographic and air travel data for the numerical simulations. Three distinct origindestination pairs are considered to understand the role of the different characteristics of the regions in the propagation of the disease, and to demonstrate the effect of possible intervention strategies. The applicability of our approach is also illustrated as we fit the model to the first wave of the 2009 A(H1N1) influenza pandemic in Canada and Mexico.

Our results for the SEAIR model highlight the significance of including travel time and travel-related infection in the modeling of the spatial spread of infectious diseases: the invasion of disease free regions is highly expedited by the elevated transmission potential during transportation. To keep a region free of infection, the most efficient intervention strategy is to control the outbreak at its source. However, screening travelers as they arrive to their destination from affected areas is a potential intervention tool as well. Such an entry screening procedure is considered in the simple epidemic model for two connected regions we present in Chapter 4. Furthermore, we also propose an SIR (susceptible- 
infected-recovered) model for disease transmission in the population of individuals who travel between $r$ regions. In the multiregional model there are multiple delays, thus we extend our framework elaborated in Chapter 2 to be able to study this system.

The dynamics of compartmental disease transmission models is often characterized by the basic reproduction number, since this quantity determines the stability of the disease free equilibrium. Forward transcritical bifurcation describes the usual situation, when the infection free steady state is asymptotically stable when $\mathcal{R}_{0}$ is less than one, but when $\mathcal{R}_{0}$ exceeds unity, stability is passed to a unique endemic equilibrium, which only exists for $\mathcal{R}_{0}>1$. However, in some SIVS (susceptible-infected-vaccinated-susceptible) epidemic models with imperfect vaccine, a different behavior has been observed; that is, two positive steady states can coexist with the disease free equilibrium for some values of $\mathcal{R}_{0}$ less than one. This phenomenon, called backward bifurcation, presents a scenario when the condition $\mathcal{R}_{0}<1$ does not ensure that the disease will be eradicated, since the stable positive steady state makes it possible for the infection to persist for certain initial conditions.

In Chapter 5, we extend the simple SIVS vaccination model to two regions which are connected by transportation, and focus our attention on the special case of one-directional traveling. We assume that the inflow of non-infective travelers into a region is constant over time, moreover infecteds do not travel. Our model is an extension of the original SIVS model as it incorporates the possibility of constant transport-related inflow (i.e., immigration) of susceptible and vaccinated individuals. After giving an explicit condition for the existence of backward bifurcation and multiple endemic equilibria, we show global stability results for the model, and examine how the structure of the bifurcation diagram depends on the immigration parameters. We show that under certain conditions, increasing the inflow of non-infectives may change the direction of bifurcation from forward to backward, or vice versa. However, it is also possible to control other model parameters in a way that, independently of the immigration parameters, the system exhibits a backward or forward bifurcation, respectively.

The dissertation is based on one paper of the author, on two papers with co-author Gergely Röst, and on one paper with co-authors Gergely Röst and Jianhong Wu. These publications are the following:

- D. H. KNiPl AND G. Röst, Multiregional SIR model with infection during transportation, Biomath 1 (2012), 1209255 http://dx.doi.org/10.11145/j.biomath. 2012.09 .255 
- D. H. Knipl, Fundamental properties of differential equations with dynamically defined delayed feedback, Electron. J. Qual. Theory Differ. Equ. No. 17 (2013) pp. 1-18. http://www . math.u-szeged.hu/ejqtde/p1883.pdf

- D. H. Knipl, G. Röst And J. Wu, Epidemic Spread and Variation of Peak Times in Connected Regions Due to Travel-Related Infections - Dynamics of an Antigravity-Type Delay Differential Model, SIAM J. Appl. Dyn. Syst. 12(4) (2013) pp. 1722-1762. http://epubs.siam.org/doi/abs/10.1137/130914127

- D. H. KNIPl And G. Röst, Backward bifurcation in SIVS model with immigration of non-infectives, Biomath 2 (2013), 1312051 http://dx.doi.org/10.11145/ j.biomath.2013.12.051 


\section{Összefoglalás}

Ebben a Ph.D. értekezésben olyan nemautonóm funkcionál-differenciálegyenletekkel foglalkozunk, ahol a késleltetést tartalmazó függvényt nem lehet a változó explicit függvényeként megadni, hanem az dinamikus módon, egy másik differenciálegyenlet-rendszer megoldásán keresztül van definiálva. A 2. fejezetben a következő kezdetiérték-problémát definiáljuk:

$$
\begin{aligned}
x^{\prime}(t) & =\mathcal{F}\left(t, x_{t}\right)=f(t, x(t))+W(t, x(t-\tau)), \\
x_{\sigma} & =\varphi,
\end{aligned}
$$

ahol $t, \sigma \in \mathbb{R}, t \geq \sigma$, valamint valamely $n \in \mathbb{Z}_{+}$-ra $x: \mathbb{R} \rightarrow \mathbb{R}^{n}, f: \mathbb{R} \times \mathbb{R}^{n} \rightarrow \mathbb{R}^{n}$ és $W: \mathbb{R} \times \mathbb{R}^{n} \rightarrow \mathbb{R}^{n}$. A $C=C\left([-\tau, 0], \mathbb{R}^{n}\right)$ fázisteret a $[-\tau, 0]$ intervallumról $\mathbb{R}^{n}$-be képezó, folytonos függvények Banach-tereként adjuk meg a szupremumnormával, ahol $\tau$ rögzített pozitív konstans. A megoldás szegmensét $x_{t} \in C$-vel jelöljük, melyre fennáll az $x_{t}(\theta)=$ $x(t-\theta)$ egyenlöség bármely $\theta \in[-\tau, 0]$-ra. Ekkor $\mathcal{F}: \mathbb{R} \times C \rightarrow \mathbb{R}^{n}$, továbbá $\varphi \in C$ jelöli a rendszer állapotát a $\sigma$ kezdeti idópontban. Azt mondjuk, hogy valamely $F: \mathbb{R} \times \mathbb{R}^{j} \rightarrow \mathbb{R}^{l}$ függvény kielégíti a ( Lip) Lipschitz-feltételt az $\mathbb{R} \times \mathbb{R}^{j}$ tér minden korlátos részhalmazán $j$ és $l$ pozitív egészekre, ha fennáll az alábbi:

(Lip) Bármely $a, b \in \mathbb{R}$ és $M>0$-ra létezik $K(a, b, M)>0$ konstans, hogy

$$
\left|F\left(t, x_{1}\right)-F\left(t, x_{2}\right)\right|_{l} \leq K\left|x_{1}-x_{2}\right|_{j}, a \leq t \leq b,\left|x_{1}\right|_{j},\left|x_{2}\right|_{j} \leq M
$$

Feltesszük, hogy $f: \mathbb{R} \times \mathbb{R}^{n} \rightarrow \mathbb{R}^{n}$ folytonos és teljesíti ( ip $)$ feltételt $\mathbb{R} \times \mathbb{R}^{n}$ minden korlátos részhalmazán. Tekintjük továbbá az alábbi, közönséges differenciálegyenletre vonatkozó kezdetiérték-problémát:

$$
\begin{aligned}
& y^{\prime}(s)=g(s, y(s)), \\
& y\left(s_{0}\right)=y_{*},
\end{aligned}
$$

ahol $m \in \mathbb{Z}_{+}, y: \mathbb{R} \rightarrow \mathbb{R}^{m}$ és $g: \mathbb{R} \times \mathbb{R}^{m} \rightarrow \mathbb{R}^{m}$, továbbá $s, s_{0} \in \mathbb{R}, s \geq s_{0}$ és $y_{*} \in \mathbb{R}^{m}$. Feltesszük, hogy $g$ folytonos $\mathbb{R} \times \mathbb{R}^{m}$-en és kielégíti (Lip) feltételt $\mathbb{R} \times \mathbb{R}^{m}$ minden korlátos részhalmazán. A közönséges differenciálegyenletek általános elméletéból következik, hogy a (2.2) rendszernek létezik $y\left(s ; s_{0}, y_{*}\right)$ egyértelmú megoldása az $\left[s_{0}, s_{0}+\alpha\right]$ intervallumon valamely $\alpha>0$-ra, továbbá a megoldás folytonosan függ a kezdeti feltételektól. A következő kiegészítő feltételt tesszük: 


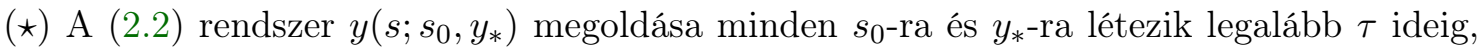
tehát az $\left[s_{0}, s_{0}+\tau\right]$ intervallumon.

Bevezetjük továbbá a $h: \mathbb{R} \times \mathbb{R}^{n} \rightarrow \mathbb{R}^{m}$ és $k: \mathbb{R} \times \mathbb{R}^{m} \rightarrow \mathbb{R}^{n}$ folytonos függvényeket, melyek kielégítik a (Lip) feltételt. A $W: \mathbb{R} \times \mathbb{R}^{n} \rightarrow \mathbb{R}^{n}$ függvényt

$$
W(s, v)=k\left(s, y_{s-\tau, v}(s)\right)=k(s, y(s ; s-\tau, h(s-\tau, v)))
$$

módon definiáljuk, ahol $y_{s_{0}, v}(s)$ jelöli (2.2) megoldását az $y_{*}=h\left(s_{0}, v\right), v \in \mathbb{R}^{n}$ speciális esetben. Mindezekböl következik, hogy $W$, mint a (2.1) rendszerben a késleltetést tartalmazó függvény, a (2.2) differenciálegyenlet-rendszer megoldásán keresztül van meghatározva. Fennáll tehát, hogy (2.1) olyan funkcionál-differenciálegyenletek általános alakját írja le, melyekben a késleltetett visszacsatolási tagot dinamikus módon adjuk meg.

Az $\mathcal{F}$ függvényre vonatkozó Lipschitz-feltétel bizonyításával nyerjük az általános létezési, egyértelmúségi, és a megoldásnak a kezdeti értékektől való folytonos függésérôl szóló tételt (2.1)-re. Ezen felül néhány, a biológiai alkalmazások szempontjából fontos eredményt is igazolunk, melyek a megoldások nemnegativitásával illetve az autonóm rendszer egyensúlyi helyzeteinek stabilitásával foglalkoznak.

A késleltetett differenciálegyenleteknek számos természettudományi és mérnöki alkalmazása van. A doktori disszertációban olyan populációdinamikai illetve járványtani modelleket vizsgálunk, melyekben a késleltetett visszacsatolási tag definiálása egy másik rendszer megoldásán keresztül történik. Az 1. fejezetben ismertetett egydimenziós modell egy populáció létszámának idôbeni változását követi azzal a feltevéssel, hogy az egyedek valamely rögzített idôre elszakadhatnak a populáció többi részétól. A 3. és 4. fejezetekben olyan késleltetett járványterjedési modellekkel foglalkozunk, melyek légiközlekedéssel összekapcsolt régiókban írják le a betegségek terjedését. Számos történelmi példa, többek között az 1918-1919-es spanyolnátha esete is illusztrálja, hogy országhatárok és óceánok sosem szabtak gátat fertőző betegségek terjedésének. A 2002-ben kitört SARS, majd a 2009-es H1N1 világjárványok világosan rámutattak, hogy a nemzetközi légiközlekedési rendszer kulcsfontosságú szerepet játszik a járványok világméretú terjedésében. A modellalkotás során feltételezzük, hogy - távoli területekrôl lévén szó - az utazás hossza nem elhanyagolható, és ugyanezen oknál fogva figyelembe vesszük azt a tényt is, miszerint a járványterjedés nem csak a szárazföldön, hanem a repülőutak alatt is zajlik. A régiókban egy késleltetett differenciálegyenlet-rendszer írja le a betegség terjedését, míg a repülöutak alatt zajló járványterjedést közönséges differenciálegyenletekkel modellezzük. Az utazás befejeztével az egyes régiókba érkező emberek csoportja mint késleltetett visszacsatolási tag jelenik meg az egyenletrendszerben. Ezt a tagot csak az utazás közbeni járványdinamika, 
vagyis az azt leíró rendszer megoldásának ismeretében lehetséges meghatározni.

A disszertáció 3. fejezetében felállítunk egy SEAIR-alapú (S - fogékony, E - látens, A - tünetmentes fertőzött, I - fertôzött tünetekkel, R - felgyógyult) modellt, mellyel két, légiközlekedéssel összekapcsolt régióban modellezzük fertôző betegségek terjedését. Mivel a populáció tagjai közötti kapcsolati hálózat nagy hatással van a járványterjedés sebességére, az embereket nem csak a betegség fázisai, de regionális hovatartozásuk szerint is megkülönböztetjük. A repülôutak alatt zajló járványterjedést egy kor-strukturált rendszer írja le, ahol a kor az utazás megkezdése óta eltelt idôt jelzi. Ha figyelembe vesszük, hogy a repülőgépek utasterének zsúfoltsága miatt a megfertőződés valószínúsége jelentôsen nagyobb utazás alatt, mint átlagos körülmények között, akkor arra az érdekes következtetésre jutunk, hogy a járvány leghamarabb a kitörés helyétôl távoli területekre terjed át. Ezért modellünk jelentôsen különbözik az ún. gravitáció-típusú modellektool, melyek azon a feltevésen alapulnak, miszerint a járvány terjedési sebessége két terület között fordítottan arányos a régiók közötti távolsággal (az elnevezés a sebesség és a távolság közötti inverz kapcsolatra utal, mely a fizikai gravitáció esetében is tapasztalható). Modellünket tehát - a fentiek okán - antigravitáció-típusúnak nevezzük. Megmutatjuk, hogy a modellt leíró húszdimenziós autonóm funkcionál-differenciálegyenlet rendszer zárt alakban a (2.1) rendszerként áll elő, így alkalmazhatjuk modellünkre az általános kezdetiérték-problémára nyert eredményeket. Bemutatunk továbbá egy eljárást, melyet az elemi reprodukciós szám kiszámítására dolgoztunk ki. Módszerünk alkalmazható minden olyan rendszer esetében, melyben a késleltetett tag egy másik rendszer megoldásán keresztül van megadva. Ezután a közönséges és késleltetett differenciálegyenletekre vonatkozó, kooperatív és irreducibilis rendszerek elméletére támaszkodva leírjuk a modell betegségmentes egyensúlyának stabilitását $\mathcal{R}_{0}$ segítségével. A modellt a 2009-es A(H1N1) influenza pandémiához parametrizáljuk, a számítógépes szimulációkban valós demográfiai és légiközlekedési adatokat használunk. A járványdinamikát számos, a régiók karakterisztikájához kapcsolódó tényezô is befolyásolja, így a régiók paraméterezéséhez három, egymástól jelentősen különböző esetet tekintünk. Végül modellünket a kanadai és a mexikói morbiditási adatok alapján rekonstruált járványgörbékhez illesztjük.

Az SEAIR-modellre nyert eredményeink kiválóan illusztrálják az utazás közbeni járványdinamika figyelembevételének fontosságát: rámutattunk, hogy a repülőutak alatt jelenlevő megnövekedett fertôzési potenciál jelentősen felgyorsítja a betegség terjedését. A járvány elleni védekezés leghatékonyabb formája, ha már a járványkitörés helyén megpróbálják megakadályozni a fertôzés terjedését; ugyanakkor pandémiás helyzetben számos ország alkalmaz olyan prevenciós stratégiákat, melyek a régióba érkező fertőzött 
utazók elkülönítésére irányulnak. A disszertáció 4. fejezetében bemutatott SIR-típusú (S - fogékony, I - fertôzött, R - felgyógyult) kétrégiós modellben feltesszük, hogy a régiókba érkezôk ilyen szûrésen esnek át. Ezt követôen vizsgálunk egy másik, szintén SIR-alapú járványterjedési modellt is, melyben a populáció tagjai $r$ régió között utaznak. A modell szükségessé teszi a 2. fejezetben kidolgozott elmélet kiterjesztését több késleltetés esetére, ugyanis a különböző régiópárok közötti utazások hossza eltérô lehet.

Az elemi reprodukciós szám fontos szerepet játszik a járványterjedés dinamikájának vizsgálatában, mivel a rendszer betegségmentes egyensúlyának stabilitása $\mathcal{R}_{0}$-tól függ. A legtöbb modellben az ún. forward transzkritikus bifurkáció írja le az egyensúlyi helyzetek lokális stabilitását. Eszerint $\mathcal{R}_{0}<1$-re a rendszernek csak egy, a betegségmentes egyensúlyi helyzete létezik, mely lokálisan aszimptotikusan stabil; ugyanakkor $\mathcal{R}_{0}=1$-ben megjelenik egy stabil pozitív egyensúly, és a betegségmentes állapot instabillá válik. Egyes modelleknél ugyanakkor másfajta bifurkációs viselkedés, az ún. backward bifurkáció esete tapasztalható: ekkor a reprodukciós szám valamely egynél kisebb értékeire a rendszernek két pozitív egyensúlya is van a betegségmentes állapot mellett. Ha az endemikus egyensúlyi helyzetek valamelyike stabil, akkor - a forward bifurkációs modellek esetével ellentétben a járványterjedés megfékezéséhez már nem mindig elegendő $\mathcal{R}_{0}$-t egy alá szorítani.

A dolgozat 5. fejezetében felállítunk egy SIVS (S - fogékony, I - fertőzött, V - vakcinált) járványterjedési modellt két régióban. Feltesszük, hogy a régiók közötti utazás egyirányú, valamint a fogékonyak és vakcináltak konstans rátával érkeznek, míg a fertôzöttek nem utaznak. Ezáltal egy olyan modellre jutunk, mely az általános SIVS-modellt általánosítva figyelembe veszi a nemfertôzô egyedek bevándorlásának lehetôségét. A modellparaméterek függvényében pontosan meghatározzuk a backward illetve a forward bifurkáció fennállásának feltételét, majd a megoldások globális viselkedését is leírjuk. Ezt következően vizsgáljuk a bevándorlás hatását a bifurkációs viselkedésen. Megadhatóak azok a paramétertartományok, melyekben a bevándorlás nem változtatja meg a bifurkáció irányát. Ugyanakkor megmutatjuk, hogy bizonyos feltételek mellett a bevándorlási paraméterek növelésével a bifurkációs viselkedés forwardról backwardra, illetve fordítva irányba változik $\mathcal{R}_{0}=1$-nél.

A disszertáció a szerzô két, Röst Gergellyel közösen írt dolgozatára, egy további, Röst Gergely és Jianhong Wu társszerzőkkel közös publikációjára, valamint egy önálló közleményére épül:

- D. H. Knipl And G. Röst, Multiregional SIR model with infection during transportation, Biomath 1 (2012), 1209255 http://dx.doi.org/10.11145/j.biomath. 2012.09.255 
- D. H. Knipl, Fundamental properties of differential equations with dynamically defined delayed feedback, Electron. J. Qual. Theory Differ. Equ. No. 17 (2013) pp. 1-18. http://www.math.u-szeged.hu/ejqtde/p1883.pdf

- D. H. Knipl, G. Röst And J. Wu, Epidemic Spread and Variation of Peak Times in Connected Regions Due to Travel-Related Infections - Dynamics of an Antigravity-Type Delay Differential Model, SIAM J. Appl. Dyn. Syst. 12(4) (2013) pp. 1722-1762. http://epubs.siam.org/doi/abs/10.1137/130914127

- D. H. Knipl And G. Röst, Backward bifurcation in SIVS model with immigration of non-infectives, Biomath 2 (2013), 1312051 http://dx.doi.org/10.11145/ j.biomath.2013.12.051 


\section{Bibliography}

[1] M. E. Alexander, S.M. Moghadas, G. Röst and J. Wu, A Delay Differential Model for Pandemic Influenza with Antiviral Treatment, Bull. Math. Biol. 70(2) (2008) pp. 382-397.

[2] J. ARINo, Diseases in metapopulations, Modeling and dynamics of infectious diseases, Vol. 11 of Ser. Contemp. Appl. Math. CAM, Higher Ed. Press, Beijing (2009) pp. $64-122$.

[3] J. Arino And P. VAN Den Driessche, A multi-city epidemic model, Math. Popul. Stud. 10 (2003) pp. 175-193.

[4] D. Balcan, H. Hu, B. Goncalves, P. Bajardi, C. Poletto, J. J. Ramasco, D. Paolotti, N. Perra, M. Tizzoni, W. V. Broeck, V. Colizza and A. Vespignani, Seasonal transmission potential and activity peaks of the new influenza A(H1N1): a Monte Carlo likelihood analysis based on human mobility, BMC Medicine 7(45) (2009)

[5] O. V. Baroyan, L. A. Rvachev, U. V. Basilevsky, V. V. Ermakov, K. D. Frank, M. A. Rvachev and V. A. Shashkov, Computer modelling of influenza epidemics for the whole country (USSR), Adv. in Appl. Probab. 3(2) (1971) pp. 224-226.

[6] S. Bonhoeffer and R. R. Regoes, Emergence of Drug-Resistant Influenza Virus: Population Dynamical Considerations, Science 312(5772) (2006) pp. 389-391.

[7] F. Brauer, Backward bifurcations in simple vaccination models, J. Math. Anal. Appl. 298 (2004) pp. 418-431.

[8] F. BRAUER, Backward bifurcations in simple vaccination/treatment models, Journal of Biological Dynamics 5(5) (2011) pp. 410-418.

[9] F. Carrat, E. Vergu, N. M. Ferguson, M. Lemaitre, S. Cauchemez, S. Leach AND A. J. Valleron, Time lines of infection and disease in human 
influenza: a review of Volunteer challenge studies, Am. J. Epidemiol. 167 (2008) pp. $775-785$.

[10] J. Cui, Y. Takeuchi And Y. Saito, Spreading disease with transport-related infection, J. Theoret. Biol. 239 (2006) pp. 376-390.

[11] O. Diekmann And J. A. P. Heesterbeek, Mathematical epidemiology of infectious diseases: model building, analysis and interpretation, Chichester, UK: Wiley (2000)

[12] O. Diekmann, J. A. P. Heesterbeek and J. A. J. Metz, On the definition and computation of the basic reproduction ratio $\mathcal{R}_{0}$ in models for infectious diseases in heterogeneous populations, J. Math. Biol. 28 (1990) pp. 365-382.

[13] O. Diekmann, J. A. P. Heesterbeek and M. G. Robert, The construction of next-generation matrices for compartmental epidemic models, J. R. Soc. Interface 7 (2010) pp. 873-885. first published online 5 November 2009

[14] H. Dulac, Points Singuliers des Equations Differentielles, Mem. Sci. Math., Vol. 61, Gauthier-Villars (1934)

[15] J. Dushoff, W. Huang and C. Castillo-Chavez, Backward bifurcations and catastrophe in simple models of total diseases, J. Math. Biol. 36 (1998) pp. 227-248.

[16] ECDC, Risk Assessment Guidelines for Infectious Diseases Transmitted on Aircraft http://ecdc.europa.eu/en/publications/Publications/0906_TER_Risk_ Assessment_Guidelines_for_Infectious_Diseases_Transmitted_on_Aircraft. pdf (2009)

[17] L. Edelstein-Keshet, Mathematical models in biology, The Random House/Birkhäuser Mathematics Series, Random House, Inc., New York (1988)

[18] A. B. Gumel, Causes of backward bifurcations in some epidemiological models, J. Math. Anal. Appl. 395(1) (2012) pp. 355-365.

[19] K. P. Hadeler and C. Castillo-Chavez, A core group model for disease transmission, Math. Biosci. 128 (1995) pp. 41-55.

[20] K. P. Hadeler And P. Van Den Driessche, Backward bifurcation in epidemic control, Math. Biosci. 146 (1997) pp. 15-35.

[21] J. K. Hale, Ordinary differential equations, RE Krieger Pub. Co., Huntington, NY, 46. (1980) 
[22] P. Hartman, Ordinary differential equations, Classics In Applied Mathematics, Vol. 38, SIAM (2002)

[23] M. W. Hirsch And H. Smith, Chapter 4 Monotone Dynamical Systems, in: A. Cañada, P. Drábek and A. Fonda, Editor(s), Handbook of Differential Equations: Ordinary Differential Equations Vol. 2, North-Holland, Amsterdam (2006) pp. 239-357.

[24] K. Khan, J. Arino, F. Calderon, A. Chan, M. Gardam, C. Heidebrecht, W. Hu, D. A. Janes, M. Macdonald, J. Sears, P. Raposo and S. Wang, The BIO.DIASPORA Project: an analysis of Canada's vulnerability to emerging infectious disease threats via the global airline transportation network, Technical report, St. Michael's Hospital, Toronto, ON, Canada (2009) available online from: http://www.biodiaspora.com

[25] K. Khan, J. Arino, W. Hu, P. Raposo, J. Sears, F. Calderon, C. HeideBrecht , M. Macdonald, J. Liauw, A. Chan and M. Gardam, Spread of a novel influenza A (H1N1) virus via global airline transportation, N. Engl. J. Med. 361 (2009) pp. 212-214.

[26] C. M. Kribs-Zaleta, Center manifolds and normal forms in epidemic models, in: C. Castillo-Chavez, S. Blower, D. Kirschner, P. van den Driessche, A. A. Yakubu (Eds.), Mathematical Approaches for Emerging and Re-emerging Infectious Diseases: An Introduction, Springer-Verlag (2001) pp. 269-286.

[27] C. M. Kribs-Zaleta and J. X. Velasco-Hernández, A simple vaccination model with multiple endemic states, Math. Biosci. 164 (2000) pp. 183-201.

[28] J. Liu, J. Wu And Y. Zhou, Modeling disease spread via transport-related infection by a delay differential equation, Rocky Mountain J. Math. 38(5) (2008) pp. 15251540 .

[29] X. Liu And Y. Takeuchi, Spread of disease with transport-related infection and entry screening, J. Theoret. Biol. 242 (2006) pp. 517-528.

[30] I. M. Longini JR., M. E. Halloran, A. Nizam and Y. Yang, Containing pandemic influenza with antiviral agents, Am. J. Epidemiol. 159 (2004) pp. 623-633.

[31] L. MARkus, Asymptotically autonomous differential systems, in: S. Lefschetz (Ed.), Contributions to the Theory of Nonlinear Oscillations III, in: Ann. Math. Stud., Vol. 36, Princeton Univ. Press (1956) pp. 17-29. 
[32] Mexican Social Security Institute http://www.imss.gob.mx/english/ Pages/default.aspx

[33] Y. NAKATA, On the global stability of a delayed epidemic model with transport-related infection, Nonlinear Anal. Real World Appl. 12(6) (2011) pp. 3028-3034.

[34] Y. NAKATA And G. RÖst, Global analysis for spread of infectious diseases via transportation networks, to appear in J. Math. Biol. (2014)

[35] Public Health Agency of Canada http://www.phac-aspc.gc.ca/fluwatch/ index-eng.php

[36] Sh. Ruan, W. Wang And S. A. Levin, The effect of global travel on the spread of SARS, Math. Biosci. Eng. 3 (2006) pp. 205-218.

[37] L. A. Rvachev And I. M. Longini JR., A mathematical model for the global spread of influenza, Math. Biosci. 75(1) (1985) pp. 3-22.

[38] H. L. Smith, An Introduction to Delay Differential Equations with Applications to the Life Sciences, Springer (2010)

[39] H. L. Smith, Monotone Dynamical Systems: An Introduction to the Theory of Competitive and Cooperative Systems, AMS (1995)

[40] Statistics Canada: Canada's national statistical agency http://www. statcan.gc.ca

[41] M. Suzuki And H. Matsunaga, Stability criteria for a class of linear differential equations with off-diagonal delays, Discrete Contin. Dyn. Syst. 24 (2009) pp. 13811391.

[42] Y. Takeuchi, X. LiU and J. CuI, Global dynamics of SIS models with transportrelated infection, J. Math. Anal. Appl. 329 (2007) pp. 1460-1471.

[43] H. R. Thieme, Asymptotically autonomous differential equations in the plane, Rocky Mountain J. Math. 24 (1994) pp. 351-380.

[44] H. R. Thieme and C. Castillo-Chavez, Asymptotically autonomous epidemic models, in: O. Arino, D. Axelrod, M. Kimmel, M. Langlais (Eds.), Mathematical Population Dynamics: Analysis of Heterogeneity, Vol. 1, Theory of Epidemics, Wuerz (1995) pp. 33-50.

[45] A. R. Tuite, J. Tien, M. Eisenberg, D. J. D. Earn, J. Ma and D. N. Fisman, Cholera epidemic in Haiti, 2010: using a transmission model to explain spatial spread 
of disease and identify optimal control interventions, Annals of Internal Medicine 154(9) (2011) pp. 593-601.

[46] A. R. Tuite, A. L. Greer, M. Whelan, A. L. Winter, B. Lee, P. Yan, J. Wu, S. Moghadas, D. Buckeridge, B. Pourbohloul and D. N. Fisman, Estimated epidemiologic parameters and morbidity associated with pandemic H1N1 influenza, CMAJ, 182 (2010) pp. 131-136.

[47] P. VAn Den Driessche And J. Watmough, Reproduction numbers and subthreshold endemic equilibria for compartmental models of disease transmission, Math. Biosci. 180 (2002) pp. 29-48.

[48] C. P. van der Weijden, M. L. Stein, A. J. Jacobi, M. E. E. Kretzschmar, R. Reintues, J. E. van Steenbergen and A. Timen, Choosing pandemic parameters for pandemic preparedness planning: A comparison of pandemic scenarios prior to and following the influenza A(H1N1) 2009 pandemic, Health Policy 109 (2013) pp. 52-62.

[49] B. Wagner, B. Coburn and S. Blower, Calculating the potential for withinflight transmission of influenza A (H1N1), BMC Medicine 7(81) (2009)

[50] W. Wang And X. Q. Zhao, An age-structured epidemic model in a patchy environment, SIAM J. Appl. Math. 65(5) (2005) pp. 1597-1614.

[51] WHO, Severe acute respiratory syndrome (SARS): Status of the outbreak and lessons for the immediate future, WHO report, Geneva, May 20, 2003, available online at http://www.who.int/csr/media/sars_wha.pdf

[52] WHO Global influenza Virological SuRveillance http://www. who.int/ gho/epidemic_diseases/influenza/virological_surveillance/en/index.html 